\title{
Copyright
}

by

Shalonda Estelle Horton

2015 
The Dissertation Committee for Shalonda Estelle Horton Certifies that this is the approved version of the following dissertation:

\section{FACTORS INFLUENCING DIETARY FAT INTAKE AMONG BLACK EMERGING ADULTS}

\section{Committee:}

Gayle Timmerman, Supervisor

$$
\text { Gayle Acton }
$$

Adama Brown

Marc Musick

Lynn Rew 


\title{
FACTORS INFLUENCING DIETARY FAT INTAKE AMONG BLACK EMERGING ADULTS
}

\author{
by \\ Shalonda Estelle Horton, B.S.N.; M.S.N. \\ Dissertation \\ Presented to the Faculty of the Graduate School of \\ The University of Texas at Austin \\ in Partial Fulfillment \\ of the Requirements \\ for the Degree of
}

Doctor of Philosophy

The University of Texas at Austin

May 2015 


\section{Dedication}

This dissertation is dedicated to three meaningful gifts in my life: my husband Daryl Lee Horton and both of my grandmothers, the late Annie Will Fielder and Johnnie Mae Brazzell.

- Daryl, I love you more than words can express. Helen Keller once said, "The best and most beautiful things in the world cannot be seen or even touched-they must be felt with the heart." I hope your heart feels my love for you, and I am blessed to be loved by you. Thank you for loving me like Christ loves the church (Ephesians 5:25). It is because of your sacrificial love that I have made it to this point.

- Granny Annie, although you went to your eternal home in heaven on April 4, 2013, I will continue your legacy of faith, love, and service to others. Thank you for teaching me that my life is a gift from God and my gift to Him is my life.

- Granny Johnnie Mae, little did I know as a little girl that me helping you with your insulin injections set me on my journey towards nursing. It was on the day you left for your eternal home in heaven on June 22, 1984 that I decided to become a healthcare professional. Thank you for reminding me that God uses all things for good. 


\section{Acknowledgements}

Foremost, I praise God for allowing me the opportunity to serve Him and make a difference in the lives of others through research! I trust you to complete the good work you have started in me (Philippians 6:1).

The African Proverb, which states "It takes a village to raise a child", does not only apply to children. As an adult, I also need continued support to reach my destiny planned by God. I am grateful of the love and support from my "village family". Thank you for believing in me, even when I did not believe in myself, and helping me find strength in God (1 Samuel 23:16): Daryl Horton, Jeff and Diane Brazzell, David and Faye Horton, Mark and Tywanna Jones, Marlysia Earls, David and Jodi Horton, Emma Horton, Aaron and Cillia Smith, and Andre and Lisa Horton, Adam and April Horton. To my aunts, uncles, and cousins, thank you for also graciously investing in me as I chase my dreams.

My heartfelt gratitude goes to my advisor and mentor, Dr. Gayle Timmerman. Thank you for being a living example of the words by Mustafa Kemal Atatürk, "A good teacher is like a candle - it consumes itself to light the way for others." I aspire to be a research scientist like you and light the way for others.

A special thank you to my committee members for their priceless investment in my development as a researcher. I truly value their expertise, feedback, and guidance in learning the research process: Dr. Gayle Acton, Dr. Adama Brown, Dr. Marc Musick, and Dr. Lynn Rew. I am blessed to have started this journey with Dr. Regina Johnson, thank you for expecting the best from me and being a wonderful mentor. 
My dearest friends lived out the biblical words of "a friend loves at all times" (Proverbs 17:17a). Special thanks to those I love like sisters: Dr. Trinia Cain, Erica Henry, Patt Jackson, Dr. Mandeep Kaur, Dana Mays, Linda Murphy, Dr. Rachelle Simmons, Dr. Ana Todd, Dr. Deidra Turner, Dr. Janiece Walker, and Lynette Williams. There is no way to recall the countless prayers, words of encourage, gifts, and assistance with recruitment from my "church family" at Mt. Zion Baptist Church. I love you and I am beyond blessed to be a part of Mt. Zion! My sincerest thanks to Pastor G. V. Clark, Mayola \& Brittany Toliver, Adrian Rhae Fowler, Linda E. Banks Knoeppel, Sydney Merriwether, Shelbi Mitchell, Mishon Davis, Tommie Mercer, Victoria Fletcher, Harold Flenoy II, Alesia Levine, Nancy Worlds, and my Sunday School Class.

I will always treasure the endless support I received from my University of Texas Austin friends (nerdy litter mates) and colleagues, who encouraged me, served as writing partners, and offered listening ears: Dr. Julie Zuniga, Dr. Lisa Sumlin, Dr. Tiffany Ricks, Dr. Elizabeth Perry, Dr. Theresa Garcia, Darlene Wilson, Ellen Martin, Janet Morrison, Marian Morris, Jackie Dingley, Jessica Taylor, LaTashia Kiel, Dr. Carol Gaskamp, Dr. Lorraine Walker, Dr. Carol Taxis, Dr. Alex Garcia, Dr. Deborah Volker, Dr. Karen Johnson, Dr. Carolyn Mueller, Dr. Pat Carter, Dr. Sook Jung Kang, Dr. Sarah Peters, Dr. Sherry Hendrickson, Tracy Deschuk, Charla Carrington, Polly Jones, Mickey Gonzales, Christina Jarvis, Dr. Patricia Castaneda-English, Ruth Brady, Tahryn Hughes, and Jimmy Eaddy.

Community partners and friends assisted me in making lasting connections in the community, recruiting participants, and offering guidance on outside agencies' IRB 
process: Leon \& Stephanie McCowan, New Hope Baptist Church (Weatherford, Texas), Dr. Charles Gramlich (Chair of the Xavier University IRB), Dr. Marguerite Giguette (Associate Vice-President for Academic Affairs at Xavier University), Dr. Jaya K. Soni (Director of Institutional Planning, Research and Assessment at Huston-Tillotson University), Dr. Kyle Boone (Director of Campus Life at Huston-Tillotson University), Ann Gosey (Ann's Private Hair Cuts), and Andy Cyphers (96.3 FM radio station).

I am grateful for the expert guidance (i.e., dissertation formatting, survey design, multiple regression, SPSS, and printing) I received from Bob Penman, Eunjin Seo, Robert Bellville, Denny Meredith-Orr (ZipSurvey.com), and Dr. Timothy Keith.

This study was possible because of financial research support and educational scholarships from donors, such as the Good Samaritan Foundation, Lee \& Joe Jamail Dean's Endowment for Excellence Scholarship, Sigma Theta Tau (Epsilon Theta Chapter), Rew Family Graduate Research Fellowship, Texas Public Health Association Scholarship, Louis W. Rase and Sophie Braun Rase Scholarship, and Texas Graduate Nurses Association Scholarship. There is no way to express how much I appreciate your investment in my research career, Thank You!

Lastly, this study would not be possible without the participants. George Elliston once said, "How beautiful a day can be when kindness touches it!" Many thanks to the participants who touched my life with kindness when they completed the survey. 


\title{
Factors Influencing Dietary Fat Intake Among Black Emerging Adults
}

\author{
Shalonda Estelle Horton, Ph.D. \\ The University of Texas at Austin, 2015
}

Supervisor: Gayle Timmerman

Currently more than $1 / 3$ of the adult U.S. population is obese. Obesity has been linked to dietary fat intake (DFI). Black emerging adults have a higher prevalence of obesity and DFI compared to other races/ethnicities, which increases their risks for cardiovascular disease. In order to reduce risks for long-term chronic illnesses linked to lifestyle choices, it is important for Black emerging adults to develop healthy eating habits while transitioning into adulthood.

The purpose of this study was to examine the influence of individual characteristics (gender, body mass index, income adequacy, and religious commitment) and behavior-specific cognitions and affect (perceived barriers for healthy eating, perceived self-efficacy related to managing dietary fat intake, perceived family social support, perceived friend social support, and perceived generalized stress) on the behavior outcome of DFI. Pender's Health Promotion Model was the theoretical framework that guided this study.

A convenience sample of 251 participants, recruited from sites frequented by Black emerging adults, such as universities, churches, and social media, used a web link to complete an online self-report survey regarding factors that influence their DFI. The viii 
survey contained a demographic questionnaire and eight established instruments, which measured the previously mentioned individual characteristics, behavior-specific cognitions and affect, and DFI.

Gender $\left(r_{p b}=-.22, p<.001\right)$ (higher fat intake among males), perceived barriers for healthy eating $(r=.32, p<.001)$, and perceived self-efficacy related to dietary fat intake $(r=-.33, p<.001)$ were the only variables studied that were significantly correlated with DFI. These variables were also significant predictors for DFI, explaining $20.1 \%$ of the variance. Gender had the greatest effect on DFI $(b=-5.671, p=.000)$. Religious commitment influenced the effects of perceived stress on dietary fat intake. This study addresses the gap in the literature by examining factors influencing DFI among Black emerging adults, an understudied population. Findings from this study may lead to culturally age-appropriate and gender-specific interventions to help Black emerging adults adopt and maintain healthy lifestyles well into their older adult years. 


\section{Table of Contents}

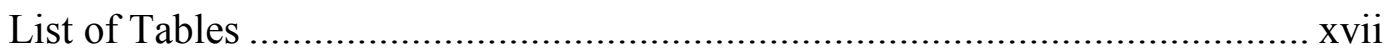

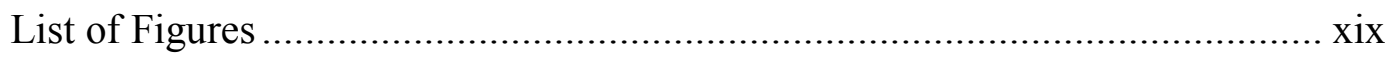

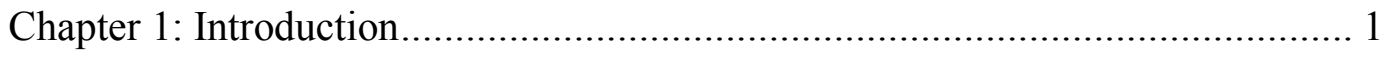

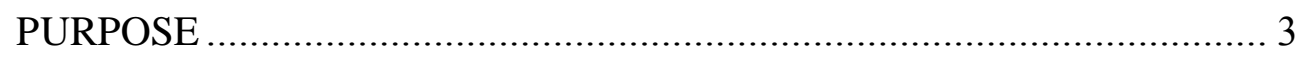

BACKGROUND AND SIGNIFICANCE ……….................................... 4

RESEARCH QUESTIONS................................................................... 10

CONCEPTUAL/THEORETICAL FRAMEWORK …............................. 11

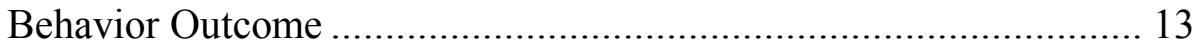

Individual Characteristics and Experiences …………..................... 14

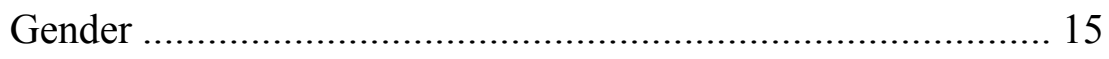

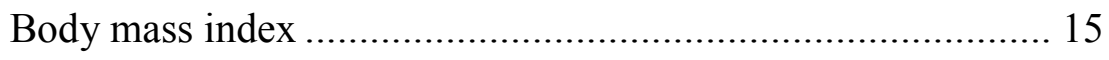

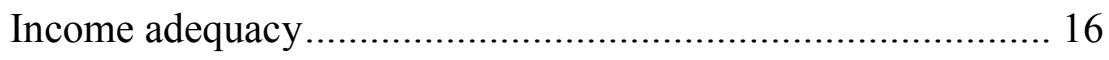

Religious commitment.......................................................... 17

Behavior-Specific Cognitions and Affect ........................................... 18

Perceived barriers ................................................................ 19

Perceived self-efficacy ………………………………...... 19

Interpersonal influences........................................................... 20

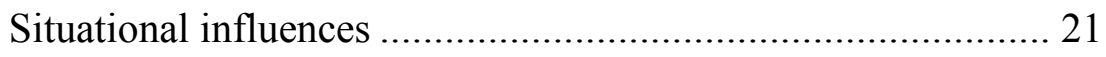

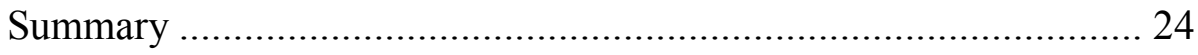

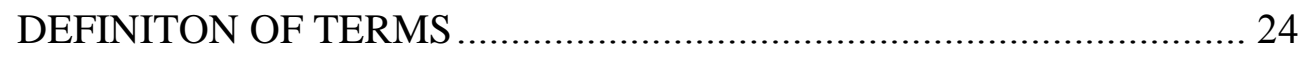

Black Emerging Adults .............................................................. 24

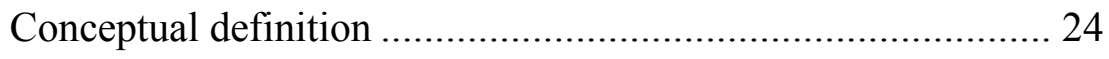

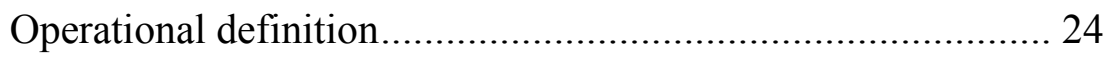

Behavior Outcome: Managing Dietary Fat Intake............................. 24

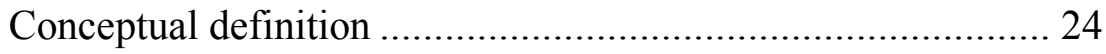

Operational definition.......................................................... 24

Individual Characteristics and Experiences ……………………..... 25

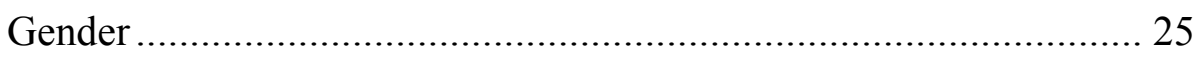




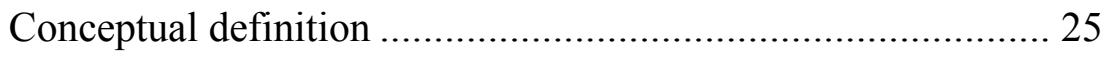

Operational definition ............................................................ 25

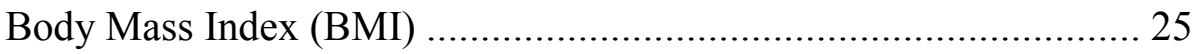

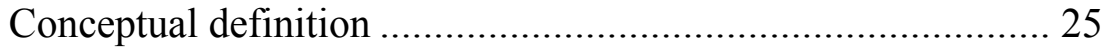

Operational definition ............................................................. 25

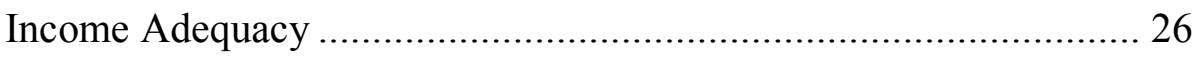

Conceptual definition ........................................................... 26

Operational definition ......................................................... 26

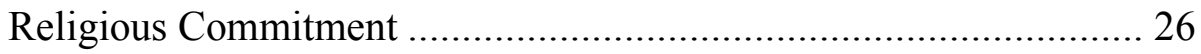

Conceptual definition ......................................................... 26

Operational definition ......................................................... 26

Perceived Barriers for Healthy Eating …………………………..... 26

Conceptual definition .......................................................... 26

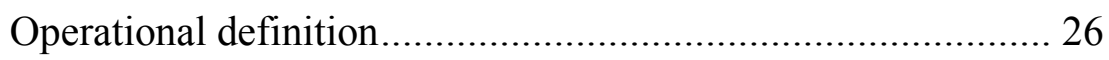

Perceived Self-efficacy Related to Dietary Fat Intake ……................ 26

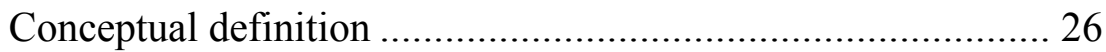

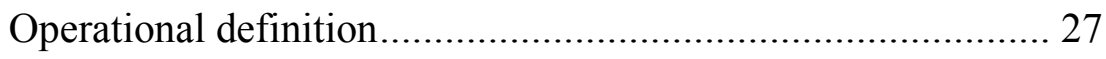

Perceived Family Social Support.................................................... 27

Conceptual definition .............................................................. 27

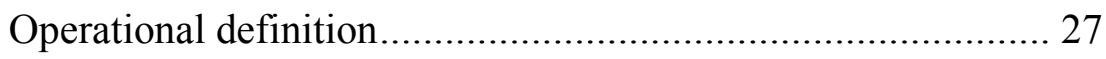

Perceived Friend Social Support …………………………………... 27

Conceptual definition ……………………………………..... 27

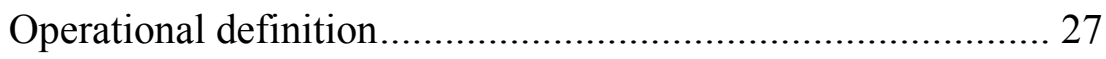

Perceived Generalized Stress ......................................................... 27

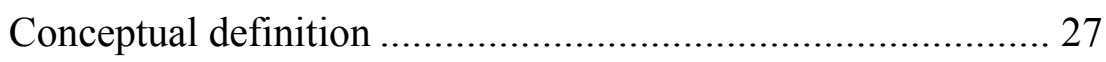

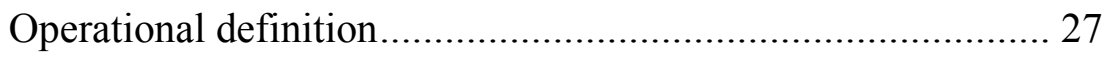

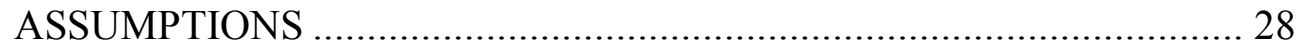

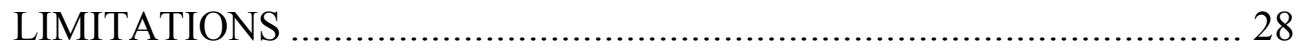

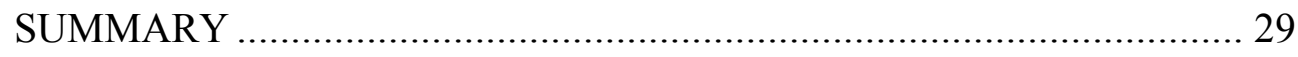




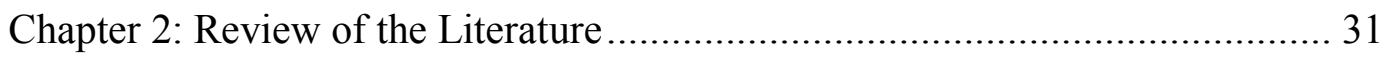

EMERGING BLACK ADULTS AND DIETARY FAT INTAKE ............. 31

DIETARY FAT INTAKE AND CULTURAL FOOD INFLUENCES ...... 33

FACTORS IMPACTING DIETARY FAT INTAKE ………………….... 36

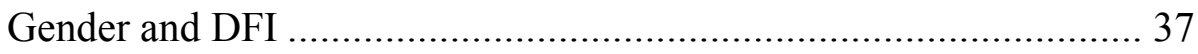

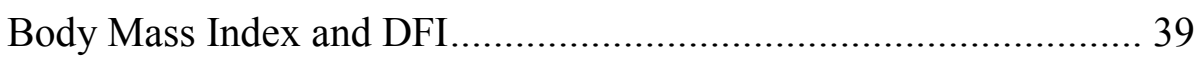

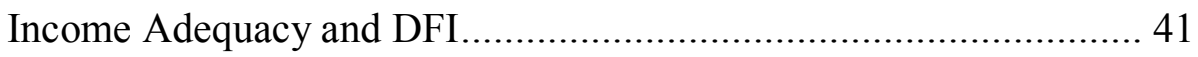

Religious Commitment and DFI..................................................... 45

Perceived Barriers for Healthy Eating and DFI.................................. 49

Perceived Self-Efficacy and DFI .................................................. 52

Perceived Social Support and DFI.................................................. 55

Perceived Generalized Stress and DFI................................................. 60

MODIFYING AND MEDIATING FACTORS OF DFI............................. 64

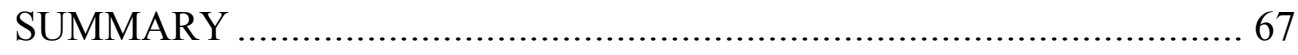

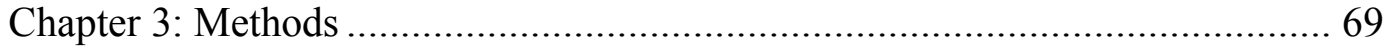

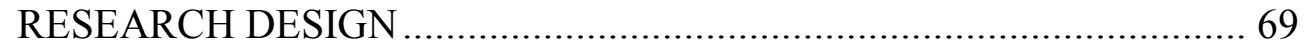

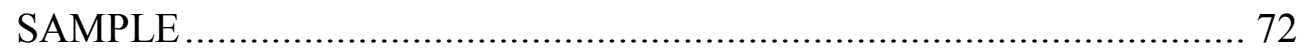

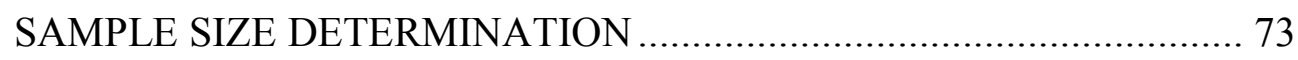

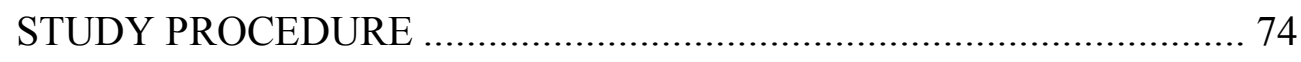

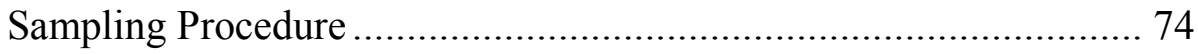

Data Collection Procedures ……………………………………..... 82

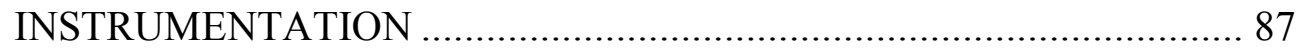

Block Dietary Fat Screener (BDFS) ……………………………... 88

Demographic Questionnaire ............................................................ 91

Body Mass Index (BMI) …………………………..................... 91

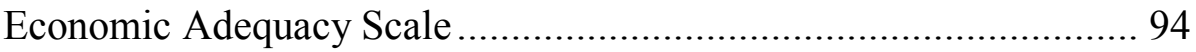

Intrinsic Religiosity (IR) Subscale of the Duke University Religion Index 96

Healthy Foods and Snacks-Barriers Subscale on the Motivators of and Barriers to Health-Smart Behaviors Inventory .... 100 
Resisting Relapse Subscale of the Self-Efficacy for Eating Behaviors

Scale 104

Family Support for Heart Healthy Eating Habits Scale ..................108

Friend Support for Heart Healthy Eating Habits Scale....................111

Perceived Stress Scale (PSS) ...............................................114

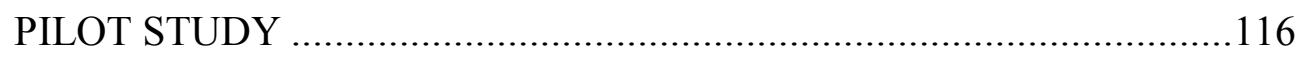

Sample in the Pilot Study ...................................................116

Procedure in the Pilot Study ...................................................118

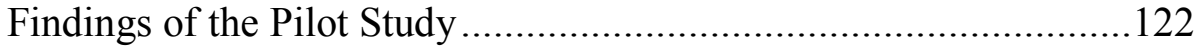

Current Study: Instruments and Reliability ...............................132

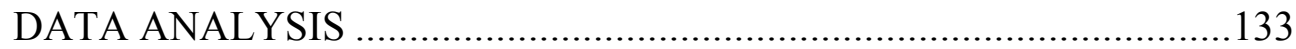

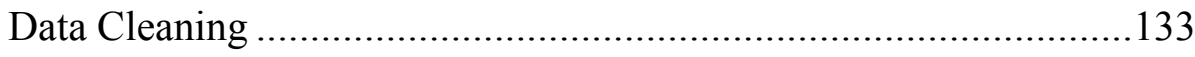

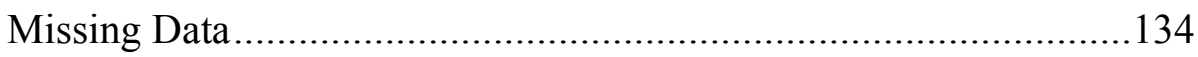

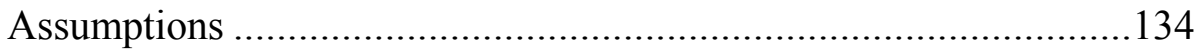

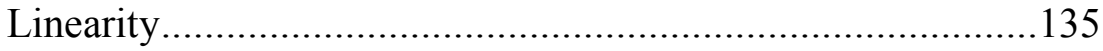

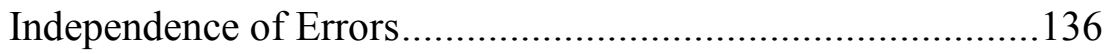

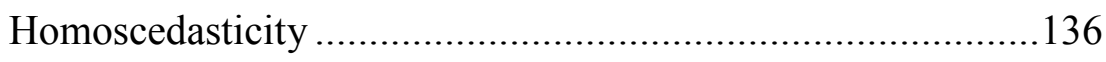

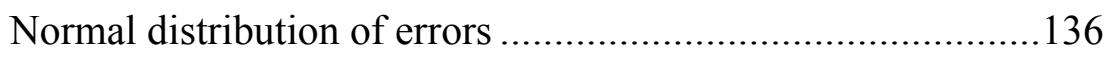

Multicollinearity............................................................ 136

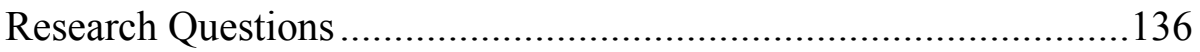

Research Question 1 .................................................136

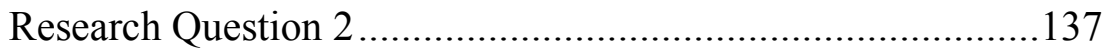

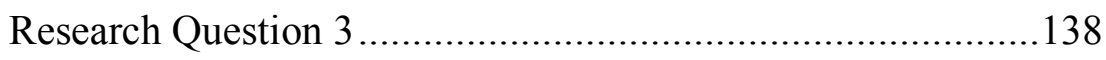

Research Question 4 ....................................................139

Research Question 5 ....................................................141

Research Question 6...................................................142

PROTECTION OF HUMAN SUBJECTS .......................................144

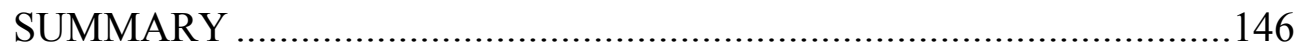

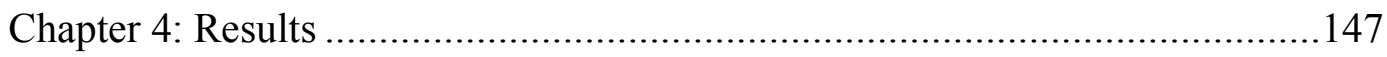

Testing Assumptions .................................................... 147 


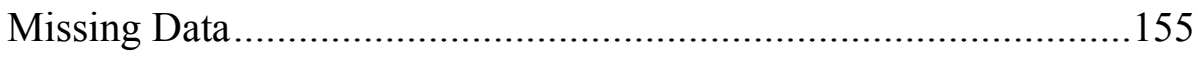

Imputations for Missing Data ..........................................................156

Mahalanobis Distance and Cook's Distance .....................................156

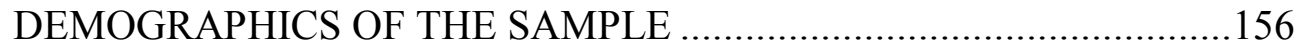

DESCRIPTIVE ANALYSES OF THE STUDY VARIABLES.................161

Behavior Outcome (Dietary Fat Intake)...........................................163

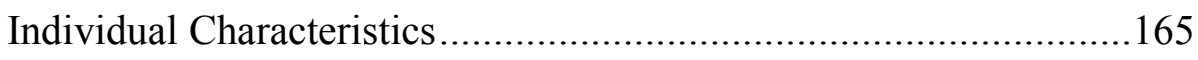

Body Mass Index...................................................................165

Religious Commitment ..........................................................167

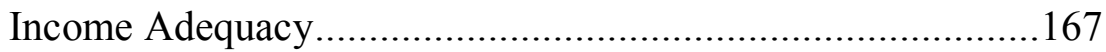

Behavior-specific Cognitions and Affect........................................167

Perceived Barrier for Healthy Eating .........................................167

Perceived Self-efficacy Related to Managing DFI ...................168

Perceived Family Support.......................................................168

Perceived Friend Support .........................................................168

Perceived Stress ...............................................................169

Differences between Paper and Online Survey ..................................169

FINDINGS RELATED TO THE RESEARCH QUESTIONS ...................171

Research Question 1 ..............................................................171

Research Question 2 ………………………………........174

Research Question 3 ……...................................................178

Research Question 4 …………………………………….......182

Research Question 5 ……...................................................190

Research Question 6...............................................................194

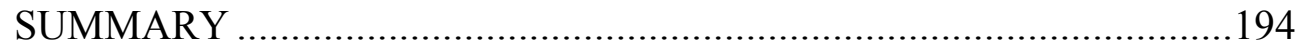

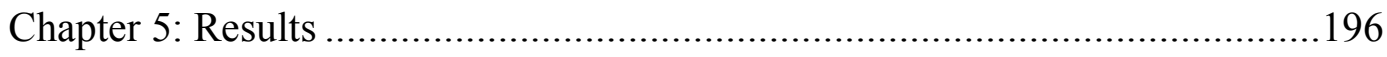

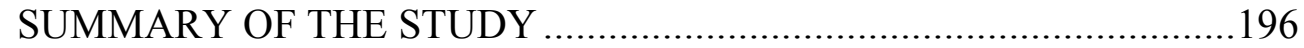

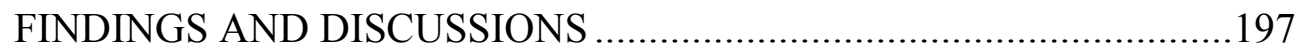

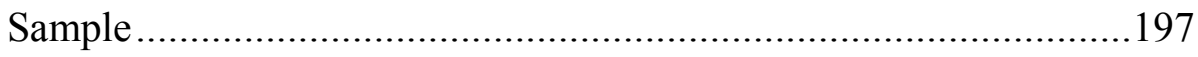

Descriptive Findings and Research Questions ..................................203 xiv 
Outcome Variable: Behavior Outcome-Dietary Fat Intake......203

Research Questions ..........................................................205

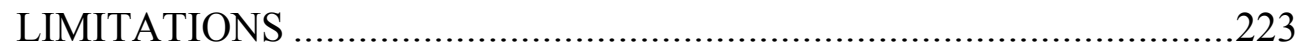

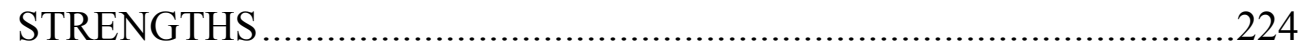

NURSING IMPLICATIONS AND RECOMMENDATIONS ................225

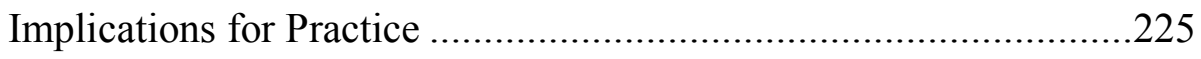

Recommendations for Future Research ....................................227

Recommendations for Theory Development..............................230

Recommendations for Health Policy ........................................2232

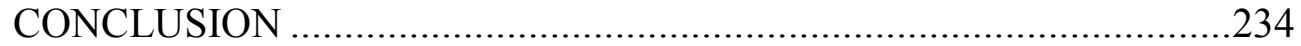




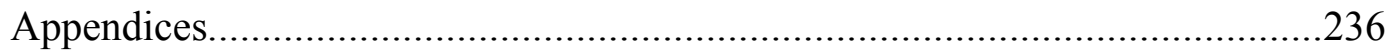

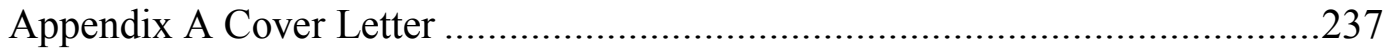

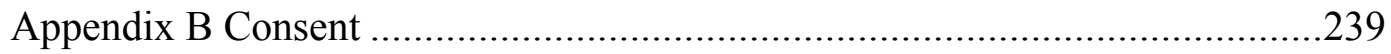

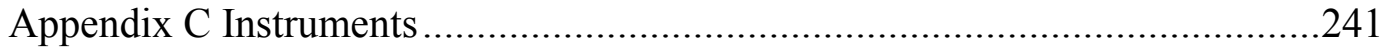

Appendix D Permission to Use Instruments .................................................22

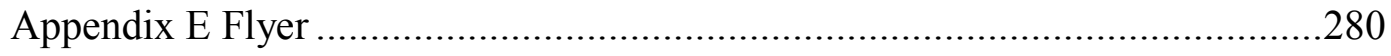

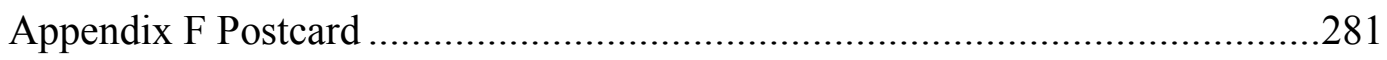

Appendix G Email Message to Organizations/Businesses..................................282

Appendix H Email Message for Fat Screening Results ....................................283

Appendix I Email Message for Mental Health Resources ................................284

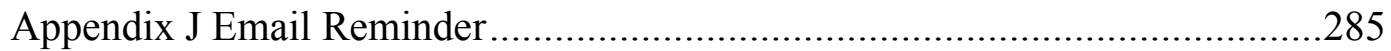

Appendix K Consent to Participate in Research (paper survey).........................286

Appendix L Healthy Eating Resources (paper survey) ....................................28

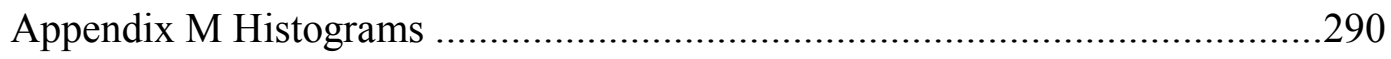

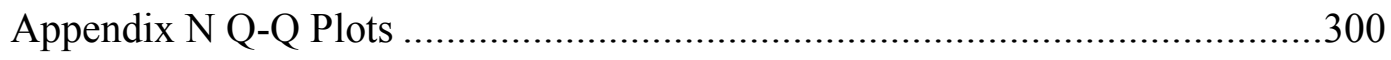

Appendix O IRB Approval for Austin Community College, Huston-Tillotson

University, and Xavier University ...........................................................309

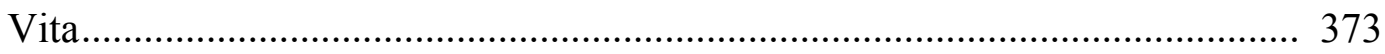




\section{List of Tables}

Table 1. Pilot Study and Dissertation Study Advertisement ............................. 81

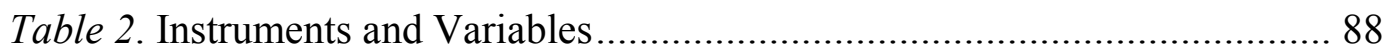

Table 3. Demographic Characteristics of Pilot Study Sample.............................118

Table 4. Correlation Matrix for Pilot Study Variables .........................................124

Table 5. Cronbach's Alpha Coefficients for the Instruments and Subscales ......125

Table 6. Cronbach's Alpha Coefficients for if Items Deleted .............................126

Table 7. Descriptive Statistics of Pilot Study Variables ...................................128

Table 8. Skewness and Kurtosis Results .........................................................149

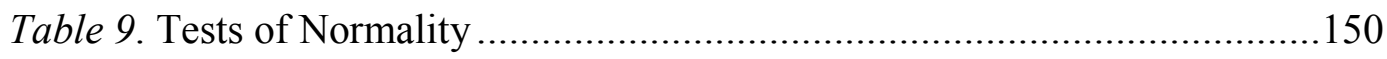

Table 10. Outliers > 3 Standard Deviations....................................................151

Table 11. Repeated Skewness and Kurtosis Results (Outliers Not Included) .....153

Table 12. Tests of Normality for Transformed Variables (Outliers Not Included) 154

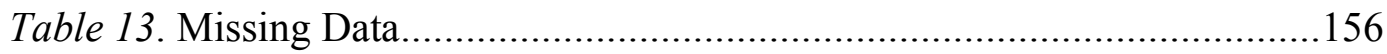

Table 15. Descriptive Statistics of Study Variables ..........................................162

Table 16. Dietary Fat Intake Categories ...........................................................163

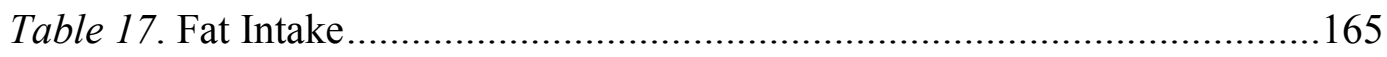

Table 18. Body Mass Index for Males and Females ...........................................166

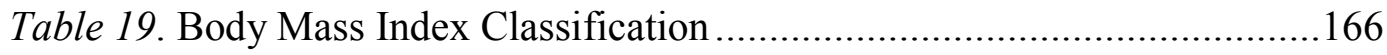

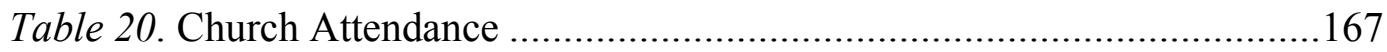

Table 23. Correlation Matrix for Study Variables ..............................................173

Table 24. Regression Weights of Gender, Perceived Barriers, and Perceived Self-

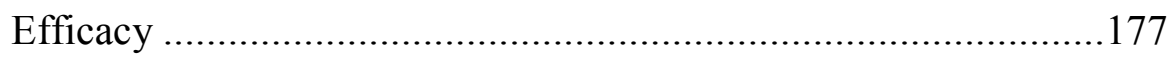

Table 25. Pooled B, $t$ and $p$ values for Multiple Imputations .............................177 
Table 26. Hierarchical Regression to Predict Dietary Fat Intake among Black

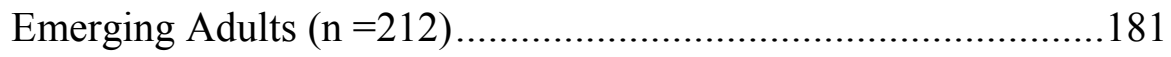

Table 27. Hierarchical Regression: Pooled B, $t$ and $p$ values for Multiple Imputations 181

Table 28. Testing Moderation Effects of Perceived Family Support by Regression 184 Table 29. Testing Moderation Effects of Perceived Family Support: Average $\mathrm{R}^{2}$, Pooled $\mathrm{B}, t$ and $p$ values for Multiple Imputations 185

Table 30. Testing Moderation Effects of Perceived Friend Support by Regression 188

Table 31. Testing Moderation Effects of Perceived Friend Support: Average $\mathrm{R}^{2}$,

Pooled $\mathrm{B}, \mathrm{t}$ and $\mathrm{p}$ values for Multiple Imputations 189

Table 32. Testing Moderation Effects of Religious Commitment by Regression 192

Table 33. Testing Moderation Effects of Religious Commitment: Average $\mathrm{R}^{2}$, Pooled $\mathrm{B}, \mathrm{t}$ and $\mathrm{p}$ values for Multiple Imputations 192 


\section{List of Figures}

Figure 1. Theoretical Framework for Factors Influencing Dietary Fat Intake Adapted from the Health Promotion Model ................................................... 23

Figure 2. Theoretical Frameworks for Moderators for Dietary Fat Intake ..........141

Figure 3. Theoretical Frameworks for Moderators for Dietary Fat Intake .........142

Figure 4. Theoretical Framework for Mediator for Dietary Fat Intake ...............143

Figure 5. Graph of Moderation Effect of Religious Commitment on Relationship between Stress and DFI..........................................................193 


\section{Chapter 1: Introduction}

According to the Surgeon General, Richard H. Carmona, obesity is the "fastestgrowing cause of disease and death in America" (Office of the Surgeon General, 2007) and has now surpassed smoking as contributing more towards the burden of disease (Jia \& Lubetkin, 2010). Currently more than $1 / 3$ (35.7\%) of the adult U.S. population is obese (Centers for Disease Control and Prevention [CDC], 2014c), with Blacks having the highest rates of obesity (49.5\%) (Flegal, Carroll, Kit, \& Ogden, 2012). Further, about 17\% (12.5 million) of youth, ages 2 to19 years, are obese (CDC, 2014a). Between the years 1976 to 2010, the prevalence of obesity increased from 5\% to $18.4 \%$ among those in the 12 to 19 years old age bracket, with Black males at $22.6 \%$ compared to $17.5 \%$ for Whites and Black females at $18.6 \%$ compared to $14.7 \%$ for Whites (Fryar, Carroll, \& Ogden, 2012). The prevalence of obesity among Blacks and Latinos is higher compared to Whites, and after controlling for family income, racial/ethnic differences in obesity persist (Freedman, 2011). According to the data collected between 1988-2008 in the National Health and Nutrition Examination Survey (NHANES), these racial/ethnic differences in obesity have increased over time among all age groups, but the greatest increase is among those who are age 2-19 years old (Freedman, 2011). As of 2014, the obesity rates among children have stabilized over the past 10 years, with some states reporting decreased rates (Levi, Segal, St. Laurent, and Rayburn, 2014). However, it is too early to call these findings promising since adult obesity rates remain extremely high across the U.S., with more than $75 \%$ of Black adults being overweight or obese (Levi et al., 2014).

Obesity is a complex problem with multiple contributing factors (Affenito, Franko, Striegel-Moore, \& Thompson, 2012; Bray, Paeratakul, \& Popkin, 2004; CDC, 
2012; Eckel \& Krauss, 1998; Hart, Tinker, Bowen, Longton, \& Beresford, 2006). One such factor is dietary fat intake. There is evidence supporting a positive association between dietary fat intake and weight gain (Binkley, Eales, \& Jekanowski, 2000; Bray et al., 2004; Bray \& Popkin, 1998; Jebb \& Prentice, 2001; Lissner \& Heitmann, 1995; Lissner, Levisky, Strupp, Kalfwarf, \& Roe, 1987). High-fat diets are palatable and often high in calories, which may lead individuals to overeat tasty foods, lose portion control, and subsequently consume more calories (Blundell, Burley, Cotton, \& Lawton, 1993; Blundell \& Macdiarmid, 1997; Bray et al., 2004; Chang, Brown, Baumann, \& Nitzke, 2008; Golay \& Bobbioni, 1997; Hu, Manson, Stampfer, Colditz, Liu, Solomon, \& Willett, 2001; Jebb \& Prentice, 2001; Lissner et al., 1987; Montmayeur \& le Coutre, 2010; Tremblay et al., 1991; Wansink, 2006; Warick \& Weingarten, 1995).

Researchers suggest that consuming an excessive amount of fat is a major nutrition concern (Lichtenstein, 1999; Lichtenstein et al., 1998). Dietary fat intake is associated with a person developing cardiovascular disease (Krauss et al., 1996; Lichtenstein et al., 1998; Nettleton, Polak, Tracy, Burke, \& Jacobs, 2009; U.S. Department of Agriculture, 2010). High dietary fat intake has also been linked to high serum cholesterol levels, weight gain, diabetes mellitus, hypertension, coronary artery disease, stroke, and certain types of cancer (Dudek, 2010; Lichtenstein et al., 1998; Siewe, 1999; Spencer, 2002).

In general Americans consume more than the recommended amount of total fat (U.S. Department of Agriculture, 2010), with Blacks consuming more total fat and saturated fat compared to other races/ethnicities (Grizzle, 2009; Harcrow, 2010; Kronsberg et al., 2003; Thompson et al., 2005; Wright \& Wang, 2010). Compared to older populations, younger populations tend to consume a higher percentage of kilocalories (kcal) from total fat than the recommended guidelines (Feunekes, de Graaf, 
Meyboom, \& van Staveren, 1998; Hampl \& Betts, 1995; Kronsberg et al., 2003; Lichtenstein et al., 1998; Sijtsma et al., 2012). Thus, further research is needed to improve the nutrition intake in younger populations of Black adults, especially when evidenced-based guidelines recommend individuals eat less fat (American Heart Association [AHA], 2011; Pender, Murdaugh, \& Parsons, 2011; U.S. Department of Agriculture, 2010; U.S. Department of Health and Human Services, 2013b).

Since obesity has been linked to fat intake and Blacks have both a higher prevalence of obesity and higher dietary fat intake compared to other races/ethnicities, the modifiable behavior of dietary fat intake is an important area of focus to promote the health of Blacks. This proposed study will examine factors that may influence dietary fat intake among Black emerging adults, ranging from 18 to 25 years old (Arnett, 2000). Results from this study may provide insight to solutions to help Black emerging adults transition into adulthood in a healthy manner, particularly in successfully managing their dietary fat intake. A framework adapted from Pender's Health Promotion Model (HPM) will be used for this proposed study (Pender, Murdaugh, \& Parsons, 2006).

\section{PURPOSE}

A goal of Healthy People 2020 is to promote the consumption of healthy diets and to achieve healthy body weight in the U.S. (U.S. Department of Health and Human Services, 2013b). Specific objectives identified in Healthy People 2020 related to dietary fat intake include reducing the intake of calories from solid fats (NWS-17.1) and reducing the intake of saturated fat (NWS-18). Consistent with the goals of Healthy People 2020, the purpose of this study is to examine select concepts from the HPM to determine the relationships among individual characteristics (gender, body mass index 
[BMI], income adequacy, and religious commitment), behavior-specific cognitions and affect (perceived barriers for healthy eating, perceived self-efficacy related to managing dietary fat intake, perceived family social support, perceived friend social support, and perceived generalized stress), and the behavior outcome of dietary fat intake. The second purpose is to examine the influence of individual characteristics (gender, BMI, income adequacy, and religious commitment) and behavior-specific cognitions and affect (perceived barriers for healthy eating, perceived self-efficacy related to managing dietary fat intake, perceived family social support, perceived friend social support, and perceived generalized stress) on the behavior outcome (DFI). The third purpose is to examine whether behavior-specific cognitions and affect (perceived family social support and perceived friend social support) moderate the effect of individual characteristics (gender, BMI, income adequacy, and religious commitment) on dietary fat intake. The fourth purpose is to examine if religious commitment moderates the effect of perceived generalized stress on dietary fat intake. The final purpose of this study is to examine if perceived generalized stress mediates the effect of religious commitment on dietary fat intake.

\section{BACKGROUND AND SIGNIFICANCE}

Emerging adults frequently consume foods high in fat (Huang, Song, Schemmel, \& Hoerr, 1994; Slining \& Popkin, 2013; Spencer, 2002). According to the 2007-2008 National Health and Nutrition Examination Survey (NHANES), the average fat intake for males was 33.6\% of total caloric intake and 33.5\% for females (Wright \& Wang, 2010). 
From 1999-2008 there was an increase in total fat intake among Blacks; for males it went from $30.5 \%$ of total calorie intake to $33.7 \%$ and females went from $32.1 \%$ to $34.4 \%$. There were not any significant trends of increasing total fat intake across other races/ethnicities (Wright \& Wang, 2010).

Researchers have also reported high fat intake and preference for high-fat foods among Blacks (Brown, Geiselman, \& Broussard, 2010; Gary et al., 2004; Grizzle, 2009; Harcrow, 2010; Horton, 2013b; Robinson \& Hunter, 2001). McGee, Richardson, Johnson, and Johnson (2014) found that Blacks did not meet the Dietary Guidelines for Americans for fat and attributed failure to meet the guidelines to consuming high calorie foods. Siewe's study (1999) found that Blacks were less likely to select foods low in fat or take steps to decrease the fat content in their foods, and they were more likely to select foods high in fat. Berg, An, and Ahluwalia (2013) conducted a study to investigate the frequency of limiting dietary fat intake among college students, ages 18-25 years. They found that students from younger and ethnic minority groups were among those who less frequently limited their dietary fat intake in the past 30 days (Berg et al., 2013). Black emerging adults are transitioning from childhood to adulthood, and it may be difficult for individuals in this age bracket to develop healthy dietary habits. They are making over 200 food decisions a day (Wansink, 2006; Wansink \& Sobal, 2007) and developing eating behaviors that may be maintained into their elderly years (Hampl \& Betts, 1995; Jensen, 2011), which supports the need to focus more attention on this population.

The high consumption of fat among Blacks could be in part due to their traditional diet (Airhihenbuwa et al., 1996; Gary et al., 2004; Hargreaves, Schlundt, \& Buchowski, 
2002; Robinson \& Hunter, 2001), which consists of more fried and high-fat foods compared to other ethnicities (Patterson, Harlan, Block \& Kahle, 1995) and less fruit, vegetables, and fiber than the recommended guidelines (Fitzgibbon et al., 2008). As total fat intake increases, the amount of saturated fat consumed generally increases. Since saturated fat raises blood cholesterol, it is frequently referred to as "bad" fat (AHA, 2011; Dudek, 2010; Montmayeur \& le Coutre, 2010). Between 2005 and 2008, according to Healthy People 2020, 15\% of adults, ages 20 years and older, had total blood cholesterol levels of $240 \mathrm{mg} / \mathrm{dL}$ or higher (U.S. Department of Health and Human Services, 2013a). Levels greater than $200 \mathrm{mg} / \mathrm{dL}$ can increase individuals' risk for CVD (AHA, 2011, 2012; Dudek, 2010; U.S. Department of Agriculture, 2010; U.S. Department of Health and Human Services, 2013b).

Both high dietary intake of fat and saturated fat have considerable health consequences. In the U.S. at least 1 in 3 adults have CVD (Roger et al., 2011), which is the leading cause of death in the U.S. (CDC, 2015a). More Black women (37.9\%) and men $(61.5 \%)$ die before the age of 75 years due to coronary heart disease compared to White women (19.4\%) and men (41.5\%) (Keenan \& Shaw, 2011). Based on 2008 mortality rates in the U.S., more than 22,000 adults die every day due to CVD (1 death every 39 seconds), and more Blacks die from CVD compared to other races/ethnicities: 390.4 per 100,000 for Black males, 287.2 per 100,000 for White males, 277.4 per 100,000 for Black females, and 200.5 per 100,000 for White females (Roger et al., 2011). In addition, $16 \%$ of total health expenditures in 2008 were due to CVD and stroke, which was more than any other health diagnosis (Roger et al., 2011, p. e209). It is estimated that 
CVD costs the U.S. about $\$ 297.7$ billion per year through direct and indirect costs (Roger et al., 2011, p. e210).

Younger populations, such as emerging adults, tend to consume foods high in fat (Anderson, Winett, Wojcik, \& Williams, 2010). Such dietary habits may increase one's risk for CVD, particularly among Black emerging adults (Brown et al., 2010; Fernandes, Arts, Dimond, Hirshberg, \& Lofgren, 2013; Hampl \& Betts, 1995; Kronsberg et al., 2003). Chronic diseases, such as CVD often begins during the emerging adult years (Bibbins-Domingo \& Peña, 2010; Brown et al., 2010; Spencer, 2002); however, adopting and maintaining healthy lifestyles, which includes lower fat intake, may decrease emerging adults' risks for CVD (Liu et al., 2012). Therefore, it is important to examine factors that influence dietary fat intake among emerging adults. Understanding the factors that contribute to dietary fat intake may provide a foundation for future work to prevent negative health consequences related to fat intake.

Most of the research regarding dietary fat intake, which included Black participants, has been descriptive and focused on the quantity of dietary fat consumed, dietary patterns, and some demographic variables (Bovell-Benjamin, Dawkins, Pace, \& Shikany, 2010; Brown et al., 2010; Bruening, Gilbride, Passannante, \& McClowry, 1999; Coates et al., 1995; Deshmuk-Taskar, Nicklas, Yang, \& Berenson, 2007; DeshmukhTaskar et al., 2009; Fitzgibbon et al., 2005; Fitzgibbon et al., 2008; Gans et al., 2009; James, 2009; Laroche, Wallace, Snetselaar, Hillis, \& Steffen, 2012; Lewis et al., 2003; Nicklas, Farris, Myers, \& Berenson, 1995; Nicklas, Myers, Reger, Beech, \& Berenson, 1998; Sharma et al., 2009; Sijtsma et al., 2012; Winkleby, Robinson, Sundquist, \& 
Kraemer, 1999). Researchers have also examined dietary fat intake in relation to other health problems (e.g., diabetes, cancer, and obesity) (Bortsov et al., 2011; Crump et al., 2006; Lagou et al., 2011). Only a few researchers have conducted studies examining dietary patterns and/or fat intake in which part of their sample consisted of adolescents and/or emerging adult populations and included Black participants (Bortsov et al., 2011; Coates et al., 1995; Frenn \& Malin, 2003; Frenn, Malin, \& Bansal, 2003; Frenn et al., 2005; Grizzle, 2009; Harcrow, 2010; Kennedy, Bowman, \& Powell, 1999; Kirkpatrick, Dodd, Reedy, \& Krebs-Smith, 2012; Lagou et al., 2011; Lewis et al., 2003; Nicklas et al., 1995, 1998; Ritchie et al., 2007; Sijtsma et al., 2012; Watters \& Satia, 2009; Winkleby et al., 1999; Zamora, Gordon-Larsen, Jacobs, \& Popkin, 2010).

The majority of the previous studies' populations were adults. If emerging adult populations were included, it is difficult to tease out the data for ages 18-25 years because such data usually overlap with other groups: children, adolescents, and adults. It is important to collect data among individuals, ages 18-25 years. Such data may help researchers and healthcare providers implement age-appropriate health-promotion interventions that assist emerging adults as they transition from childhood to adulthood and form eating behaviors that may be maintained into their elderly years (Hampl \& Betts, 1995; Jensen, 2011). Harcrow (2010) is the only researcher found to examine dietary fat among a sample that was mostly emerging adults $(M$ age $=20.58, S D=2.89)$, and $16.7 \%$ of that sample was Black. Harcrow (2010) examined the following factors: physical activity level, religiosity, spirituality, social support, and dietary fat intake. Harcrow (2010) found that religiosity, spirituality, and social support were not 
significantly related to dietary fat intake. However, behavioral religiosity $(\beta=.549, p=$ $.033)$ and functional religiosity $(\beta=.109, p=.031)$, were significantly related to dietary fat intake, each explaining $1.7 \%$ of the variance in dietary fat intake. Behavioral religiosity includes dimensions of religious denomination, religious attendance, and religious application; functional religiosity includes dimensions of religious coping and religious social support (Harcrow, 2010). Grizzle (2009) is the only researcher found to examine dietary fat using the HPM among a sample that included emerging adults, sample age ranged from 24 to 63 years $(M=41.7$ years, $S D=7.85)$. The majority of Grizzle's sample (2009) was Black (58.7\%). Grizzle's study (2009) examined the following factors: gender, BMI, barriers to healthy eating, dietary self-efficacy, occupational stress, and fat-related diet habits. Grizzle (2009) found a positive relationship between barriers to healthy eating and occupational stress $(r=.229, p<$ .001), with high barriers to healthy eating scores corresponding to high perceived occupational stress levels.

No studies, with a sample exclusively comprised of emerging adults, to date have examined the factors proposed in this study: individual characteristics (gender, BMI, income adequacy, and religious commitment), behavior-specific cognitions and affect (perceived barriers for healthy eating, perceived self-efficacy related to managing dietary fat intake, perceived family social support, perceived friend social support, and perceived generalized stress), and the behavior outcome of dietary fat intake. Understanding how all these variables relate to dietary fat intake can potentially lead to innovative interventions and programs designed to reduce fat intake in Black emerging adults. The theoretical 
framework, adapted from the Health Promotion Model, provides a guide for researchers (Pender et al., 2006) to address the health-promoting behavior of managing dietary fat intake.

\section{RESEARCH QUESTIONS}

Based on a conceptual framework adapted from the HPM (Pender et al., 2006) and the identified need for additional research on factors influencing dietary fat intake among Black emerging adults, the following research questions will be explored:

1. What are the relationships among the individual characteristics (gender, BMI, income adequacy, and religious commitment), behavior-specific cognitions and affect (perceived barriers for healthy eating, perceived self-efficacy related to managing dietary fat intake, perceived family social support, perceived friend social support, and perceived generalized stress), and dietary fat intake among Black emerging adults?

2. What are the significant predictors for dietary fat intake among the independent variables of individual characteristics (gender, BMI, income adequacy, and religious commitment) and behavior-specific cognitions and affect (perceived barriers for healthy eating, perceived self-efficacy related to managing dietary fat intake, perceived family social support, perceived friend social support, and perceived generalized stress) for Black emerging adults?

3. After controlling for individual characteristics (gender, BMI, income adequacy, and religious commitment), what are the significant predictors for dietary fat intake among Black emerging adults? 
4. Do perceived family social support and perceived friend social support moderate the effects of individual characteristics (gender, BMI, income adequacy, and religious commitment) on dietary fat intake?

5. Does religious commitment moderate the effect of perceived generalized stress on dietary fat intake?

6. Does perceived generalized stress mediate the effect of religious commitment on dietary fat intake?

\section{CONCEPTUAL/THEORETICAL FRAMEWORK}

This study used Pender's Health Promotion Model to examine factors that may influence dietary fat intake. The Health Promotion Model (HPM) has been used since the early 1980s (Pender, 1996), and the HPM has been tested in populations that consisted of emerging adults in studying condom use, physical activity/exercise, oral health, and tobacco (Dilorio, Dudley, Soet, Watkins, \& Maibech, 2000; Grubbs \& Carter, 2002; Martinelli, 1999; Morowatisharifabad \& Shirazi, 2007). The HPM has also been used in studying health-promoting behaviors in general among samples that included Black emerging adults (Brown, 2009; Callaghan, 2003, 2006; Edmonds, 2010; Grizzle, 2009; Hutchinson, 1995; Jackson, Tucker, \& Herman, 2007; Johnson, 2005; McElligott, Siemers, Thomas, \& Kohn, 2009; Wilson, 2005).

The Health Promotion Model has served as a guide to explore complex processes that encourage individuals to perform health-promoting behaviors. Pender et al. (2006) define health promotion as the behaviors performed by individuals to increase their wellbeing and health potential. The HPM illustrates how the dynamic interactions between a 
person and his/her environment can facilitate or impede engaging in health promotingbehaviors (Pender, 1996). The HPM consists of individual characteristics and experiences that influence behavior-specific cognitions and affect, which ultimately influence one's behavior outcome such as managing one's dietary fat intake (Pender et al., 2006).

Based on Pender and colleagues' conceptual framework (2006) and the review of the literature, a theoretical framework for factors influencing dietary fat intake was adapted from the HPM for this proposed study (refer to Figure 1). Select variables from the theoretical framework, which may influence the health-promoting behavior outcome of managing dietary fat intake, were examined in this study. The HPM consists of three main constructs: individual characteristics and experiences, behavior-specific cognitions and affect, and behavior outcome.

The individual characteristics and experiences construct is defined as the "unique personal characteristics and experiences of an individual that affect his/her subsequent" health actions (Pender, 1996, p. 66). According to Pender (1996), the individual characteristics and experiences construct is comprised of biological, psychological, and sociocultural factors, which may influence an individual's health actions. The biological factors examined in this study were gender and BMI. The sociocultural factors examined in this study were income adequacy and religious commitment.

The behavior-specific cognitions and affect construct is comprised of factors, which are considered "to be of major motivational significance" (Pender, 1996, p. 68) because these factors may be changed through an intervention for a desired behavior outcome (George, 2011; Pender, 1996). These factors can influence whether or not 
individuals perform health-promoting behaviors. The behavior-specific cognitions and affect factors examined in this study were perceived barriers, perceived self-efficacy, interpersonal influences (perceived family social support and perceived friend social support), and situational influences (perceived generalized stress).

The behavior outcome construct is the ending result or health promotion action performed. The behavior outcome is influenced by the aforementioned factors. The behavior outcome for this study is dietary fat intake.

\section{Behavior Outcome}

Behavior outcome refers to the definitive health promotion action you want individuals to perform (e.g., exercise and weight management) (Pender et al., 2006). The behavior outcome is usually focused on a positive health outcome among individuals (Pender, 1996), such as managing total fat intake. Managing one's total fat intake could be considered health promotion or a disease prevention action, also known as health protection (Pender et al., 2006). The "motivation for the behavior" is the determining factor in whether managing dietary fat intake is a health promotion or health protection action (Pender et al., 2006, p. 7). If individuals are managing their dietary fat intake in order to increase their well-being and health potential, then their motivation for this behavior is considered to be health promotion (Pender et al., 2006). However, if individuals are managing their dietary fat intake in order to avoid illness (e.g., CVD) and consequences connected with the illness, then their motivation for this behavior is considered to be health protection (Pender et al., 2006). Managing dietary fat intake could potentially increase one's overall health, especially in a population of emerging adults 
who may not be thinking about preventing disease. Therefore, for the purposes of this study, the behavior outcome was considered as a health promotion behavior, with the assumption that the motivation behind managing dietary fat intake was to increase one's well-being and health potential (Pender et al., 2006).

Limited research exists on the use of the HPM and dietary fat intake among emerging adult populations. To this date, research using the HPM with managing dietary fat as the behavioral outcome has been the focus of only one study. Grizzle (2009) examined "fat-related diet habits" among police officers, ages 22 to 65 years (p. 7). She found that $28.1 \%$ of the variance in "fat-related diet habits" was explained by the variables gender, age, race/ethnicity, marital status, shift assignment, benefits of healthy eating, barriers to healthy eating, and dietary self-efficacy.

This study's proposed theoretical framework suggests that the behavior outcome, managing dietary fat intake, is influenced directly and indirectly by individual characteristics and experiences (gender, BMI, income adequacy, and religious commitment) and behavior-specific cognitions and affect (perceived barriers, perceived self-efficacy, perceived family social support, perceived friend social support, and perceived generalized stress). Therefore, these factors were examined for their direct and indirect effects on dietary fat intake. In addition, this study explored select potential mediators and moderators of dietary fat intake.

\section{Individual Characteristics and Experiences}

Pender et al. (2006) list individual characteristics, such as gender, BMI and socioeconomic status that influence behavior. Individual characteristics and experiences 
thought to affect dietary fat intake included in this proposed framework are gender, BMI, income adequacy, and religious commitment. These variables have been included because of their significant correlations with healthy dietary patterns.

Gender. Gender, classified as a biological factor, is included because dietary behaviors between males and females tend to be different. For example, in general females are more knowledgeable about nutrition and likely to comply with dietary guidelines and value the importance of nutrition content of foods compared to males (Baker \& Wardle, 2003; Morse \& Driskell, 2009; Nayga, 2000; Turrell, 1997). In addition, the types of foods consumed may differ between males and females, with males consuming more high calorie foods and high fat foods (Herman \& Polivy, 2010; Hermstad, Swan, Kegler, Barnette, \& Glanz, 2010; Huang et al., 1994; Millen et al., 1997).

Body mass index. Body mass index is classified as a biological factor. Individuals who are obese may have a delayed satiety signal, prefer high-fat foods, and/or crave highfat foods, which may impede their ability to stop eating compared to lean or normal weight individuals (Detz, 2006; Drewnowski, Kurth, Holden-Wiltse, \& Saari, 1992; Liu, Gao, Liu, \& Fox, 2000; von Deneen \& Liu, 2011). Their inability to stop eating may lead to an increased consumption of dietary fat intake. In general, BMI has a positive association with unhealthy dietary behaviors (Canfi et al., 2011; Maskarinec, Novotny, \& Tasaki, 2000; Vernarelli, Mitchell, Hartman, \& Rolls, 2011), which is why it has been included in the proposed framework. Although there are mixed findings regarding the relationship between BMI and fat intake, researchers have found a positive association 
(Ledikwe et al., 2003; Miller et al., 2012; Newby et al., 2003). According to this proposed theoretical framework, BMI has a potential relationship with dietary fat intake, both directly and indirectly.

Income adequacy. Income adequacy, classified as a sociocultural factor by Pender et al. (2006), is a commonly studied individual characteristic. Having an adequate income can be a determinant in whether or not individuals consume healthy diets (Brug, 2008; Darmon \& Drewnowski, 2008; Inglis, Ball, \& Crawford, 2009; Raffensperger et al., 2010). One's level of income and the price of food can affect food choices, dietary behaviors, and the quality of food selected (Carlson \& Gould, 1994; Drewnowski \& Specter, 2004). Low incomes may not be adequate for individuals to select healthy food options, which cost more in general (Drewnowski \& Darmon, 2005). There tends to be less availability and selection of quality food among poor individuals, which places them at risk for poor nutrition (Mazur, Marquis, \& Jensen, 2003; Philip, James, Nelson, Ralph, \& Leather, 1997; U. S. House of Representatives Select Committee on Hunger, 1993, p. 20). On the other hand, high incomes may be adequate for individuals to select healthy food options. Individuals at higher income levels tend to follow recommendations for healthy dietary behaviors (e.g., consume more vegetables and have low fat intake) compared to those at lower income levels (Berrigan, Dodd, Troiano, Krebs-Smith, \& Barbash, 2003; Eyler, Haire-Joshu, Brownson, \& Nanney, 2004; Jen, Brogan, Washington, Flack, \& Artinian, 2007; Subar, Ziegler, Patterson, Ursin, \& Graubard, 1984). In addition, Eyler et al. (2004) found that education level and income were the strongest variables associated with fat intake. 
Religious commitment. Lastly, religious commitment is classified as a sociocultural factor because at the individual level there may be cultural beliefs and practices pertaining to religious commitment that may influence one's health behaviors, which is an individual characteristic. Religious beliefs may influence the way individuals live their life. As they follow their religious beliefs, they may choose to practice healthy behaviors. For example, individuals who believe their bodies are temples may choose to not smoke tobacco in order to protect their bodies/temples. Researchers may sometimes use religious terms interchangeably (e.g., religion, religiosity, spirituality, and religious commitment) and may not know how to best define or measure them (Cohen \& Koenig, 2003; Hernandez, 2011; Hyman \& Handal, 2006; Joshi, Hardy, \& Hawkins, 2009; Roberts \& Yamane, 2003; Russinova \& Cash, 2007; Takamizawa, 1999; Vachon, Fillion, \& Achille, 2009; Waldron-Perrine et al., 2011; Watson \& Nesti, 2005).

Religious commitment was selected for this study because it describes the influence of religious beliefs upon an individual's decisions and lifestyle (Koenig, McCullough, \& Larson, 2001). If individuals' religious beliefs include caring for their bodies as "temples," then they may be more likely to eat healthy foods. Healthy eating behaviors (e.g., lower fat intake) have been found among some religious groups (e.g., Seventh-Day Adventists) (Koenig et al., 2001). For the purposes of this this study, religious commitment is defined as the degree of internal commitment to one's religious beliefs, which in turn should motivate an individual's behavior (e.g., manage dietary fat intake) (Koenig et al., 2001). Some researchers have found a positive association between religious commitment and healthy diets (Chester, Himburg, \& Weatherspoon, 2006). 
Religious commitment often plays a major role among Black populations and may influence dietary fat intake behaviors. For example, in a study conducted by Schlundt and colleagues (2008), in which the sample was made of $65 \%$ Blacks $(n=1905)$, they found a small, positive relationship between fat intake and religious variables $(r=.10, p<.0001)$. On the other hand, in studies conducted by Fitzgibbon et al. (2005) with a sample of all Black women $(N=59)$, Harcrow (2010) with a sample of college students $(N=914$, Blacks 16.7\%), and Reeves, Adams, Dubbert, Hickson, and Wyatt (2012) with a sample of all Black adults $(N=2387, M$ age $=53.6$ years $)$ there were not any statistically significant relationships between religion, religiosity, spirituality, and/or religious commitment and dietary fat intake.

However, the proposed theoretical framework indicates that religious commitment may positively influence individuals' dietary fat intake. In this study, gender, BMI, income adequacy, and religious commitment may directly influence dietary fat intake and indirectly through perceived barriers, perceived self-efficacy, perceived family social support, perceived friend social support, and perceived generalized stress. Therefore, these factors were examined for their direct and indirect effects on dietary fat intake.

\section{Behavior-Specific Cognitions and Affect}

According to Pender (1996), there are factors that motivate and move individuals toward a desired health-promoting behavior or hinder them from performing the behavior. Such factors are significant because they may be modified through an intervention, thus influencing a desired behavior outcome (George, 2011; Pender, 1996), 
in this case dietary fat intake. Behavioral-specific cognitions and affect factors selected for this study are perceived barriers, perceived self-efficacy, interpersonal influences, and situational influences.

Perceived barriers. Perceived barriers are anything, whether real or perceived, that keep individuals from executing the desired behavior outcome, successfully managing their dietary fat intake (Pender, 1996). For this study it was identified as perceived barriers for healthy eating in the proposed theoretical framework. If individuals encounter significant barriers in trying to eat healthy, then they may not be successful in their attempt to manage their fat intake. For example, some researchers have identified lack of motivation and knowledge, taste preferences, access, and time as barriers for healthy eating and fat intake (Feunekes et al., 1998; James, 2004; Lloyd, Paisley, \& Mela, 1995; Neumark-Sztainer, Story, Perry, \& Casey, 1999; Timmerman, 2007). Based on Pender and colleagues' (2002) revised HPM, perceived barriers directly and indirectly (through commitment to a plan of action) influenced the behavior outcome. For this study, based on the literature and the theoretical framework, perceived barriers for healthy eating may directly influence dietary fat intake in that as barriers increase then the likelihood of individuals successfully managing their dietary fat intake decreases.

Perceived self-efficacy. Perceived self-efficacy is defined as an individual's perception of whether or not he/she has the capability to perform a specific healthpromoting behavior (e.g., successfully managing one's dietary fat intake) (Bandura, 1986; Pender et al., 2002). For this study it was identified as perceived self-efficacy related to managing dietary fat intake in the proposed theoretical framework. Self- 
efficacy cannot be generalized to all behaviors related to managing dietary fat intake (AbuSabha \& Achterberg, 1997; Clark \& Dodge, 1999). For example, an individual can have high self-efficacy for reading fat content on food labels but have low self-efficacy for changing poor dietary patterns. If individuals think that they are capable in managing their dietary fat intake, then they may consume less dietary fat. In support, researchers have found an inverse relationship between perceived self-efficacy and dietary fat intake (AbuSabha \& Achterberg, 1997; Armitage \& Conner, 1999; Grembowski et al., 1993; Nothwehr, 2004; Povey, Conner, Sparks, James, \& Shepherd, 2000; Watters \& Satia, 2009). In Pender and colleagues' (2002) revised HPM, perceived self-efficacy directly and indirectly (through commitment to a plan of action) influenced the behavior outcome. For this study, was predicted that perceived self-efficacy related to dietary fat intake would directly influence dietary fat intake in that as self-efficacy increases then the likelihood of individuals successfully managing their dietary fat intake also increases.

Interpersonal influences. Pender et al. (2006) defines interpersonal influences as the behaviors, beliefs, or attitudes of others that can affect an individual's engagement in health-promoting behaviors. Pender et al. (2006) describe multiple sources for interpersonal influences (i.e., family, peers, and healthcare providers). Such influences may help individuals perform health-promoting behaviors by establishing norms (expectations for individuals), providing social support (encouragement for individuals), and assisting in role modeling behavior (individuals learning health-promoting behaviors) (Pender et al., 2006). For this study, perceived family and friend social support were examined separately. Social support is the encouragement an individual receives through 
his/her relationships with others (Heaney \& Israel, 2008). Perceived family and friend social support are included in the framework because the encouragement individuals receive within their social settings may facilitate or hinder behaviors associated with managing dietary fat intake (Feunekes et al., 1998; Fowles, Stang, Bryant, \& Kim, 2012; Hart, Bowen, Kuniyuki, Hannon, \& Campbell, 2007; Silverman, Hecht, \& McMillin, 2002; Thrasher, Campbell, \& Oates, 2004). The revised HPM developed by Pender et al. (2002) illustrates how social support directly and indirectly (through commitment to a plan of action) influenced the behavior outcome. For this study, was predicted that perceived family and friend social support directly influenced dietary fat intake in that as each type of social support increases then the likelihood of individuals successfully managing their dietary fat intake also increases.

Situational influences. Situational influences refer to the context of one's environment, which can facilitate or hinder health-promoting behaviors related to dietary fat intake (Pender et al., 2006). The proposed theoretical framework suggests that stressful situations can become too demanding or taxing upon an individual in that he/she cannot practice health-promoting behaviors, which is why perceived generalized stress is a variable that can be included in the framework. Stress is the transactions that occur between an individual and his/her environment that become too demanding (Lyon, 2000). For this study it was identified as perceived generalized stress within the proposed framework. Although there is conflicting data in the literature regarding whether or not stress influences individuals' quality of diet (e.g., fat intake) (Wansink, 2006), it is possible for persons with high levels of stress to consume more than their recommended 
percentage of energy from fat (Fowles et al., 2012; Ng \& Jeffery, 2003) and overeat (Torres \& Nowson, 2007). In addition, stress has been associated with emotional and binge eating (Oliver, Wardle, \& Gibson, 2000; Sims et al., 2008; Wansink, 2006).

Perceived stress may become too taxing upon individuals, which may hinder their ability to manage dietary fat intake. The revised HPM developed by Pender et al. (2002) illustrates how situational influences directly and indirectly (through commitment to a plan of action) influence the behavior outcome. For this study, it was predicted that perceived generalized stress directly influenced dietary fat intake in that as it increases then the likelihood of individuals successfully managing their dietary fat intake decreases. 
Figure 1. Theoretical Framework for Factors Influencing Dietary Fat Intake Adapted from the Health Promotion Model

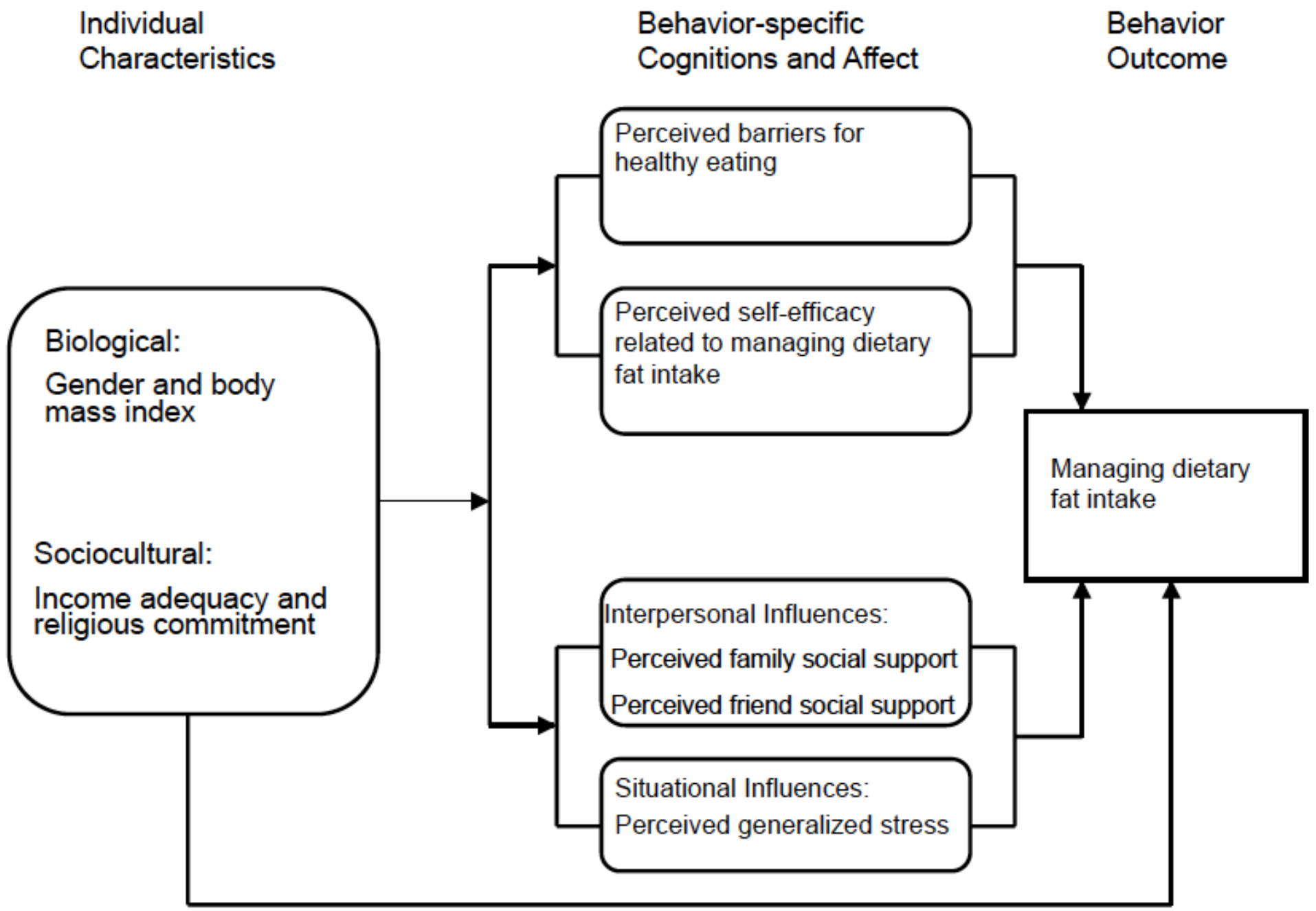




\section{Summary}

The factors included in this proposed theoretical framework are logically connected to each other. As previously stated the HPM has been used with diverse populations, some which included Black emerging adults as participants. Therefore, the HPM is appropriate to use in examining dietary fat intake among Black emerging adults.

\section{DEFINITON OF TERMS}

\section{Black Emerging Adults}

Conceptual definition: participants who are 18 to 25 years of age and selfidentify as being Black for ethnicity/race.

Operational definition: participant's response on the Demographic Questionnaire to both: 1) Do you self-identify as Black? as being "yes" and 2) How old are you? as being 18 to 25 years old. For the question regarding his/her age, the participant wrote in his/her age in years.

\section{Behavior Outcome: Managing Dietary Fat Intake}

Conceptual definition: eating habits that consist of a diet low in the percentage of total fat calories consumed in one's diet, which is total fat intake $\leq 35 \%$ of total energy intake.

Operational definition: participant's total score on the Block Dietary Fat Screener (BDFS), a 17-item food frequency instrument, was used to determine the percent of total calories from fat intake. A total score of $\leq 14$ indicates having a total fat intake of $\leq 35 \%$. 


\section{Individual Characteristics and Experiences}

For this study the individual characteristics and experiences are the personal factors considered to be predictive of health-promoting behavior related to managing dietary fat intake. Personal factors in this study can be categorized as biologic or sociocultural factors (Pender et al., 2002). Biological factors for this study were gender and BMI and sociocultural factors were income adequacy and religious commitment.

\section{Gender}

Conceptual definition: biological and physiological characteristics that categorize individuals as female or male.

Operational definition: participant's response on the Demographic Questionnaire: "What is your gender?" For the question, the participant answered in one of the following ways: 1) Male or 2) Female.

\section{Body Mass Index (BMI)}

Conceptual definition: measurement of participant's body fatness (CDC, 2015b). Operational definition: calculated using participant's self-report of height and weight on the Demographic Questionnaire. The participants' self-report for the following questions was used to calculate BMI questions: 1) "How tall are you without shoes (in feet and inches, to the nearest $1 / 2$ inch)?" and 2) "How much do you weigh without shoes (in pounds, to the nearest pound)?" The participant's self-reported height and weight was converted to kilograms and meters. These 
converted values were used to calculate $\mathrm{BMI}$ in the following formula: $\mathrm{BMI}=$ (weight in kilograms / height in meters ${ }^{2}$ ) (CDC, 2015b).

\section{Income Adequacy}

Conceptual definition: individuals' perception of whether they have enough money to meet their needs.

Operational definition: the participant's total score on the Economic Adequacy Scale (EAS).

\section{Religious Commitment}

Conceptual definition: an individual's degree of internal commitment towards his/her religious beliefs (Koenig, McCullough, \& Larson, 2001).

Operational definition: the participant's total score on the Intrinsic Religiosity (IR) subscale, which is from the Duke University Religion Index.

\section{Perceived Barriers for Healthy Eating}

Conceptual definition: anything, whether real or perceived, that the participant believes keeps him/her from successfully eating healthy foods (Pender, 1996).

Operational definition: participant's total score on the subscale called Healthy Foods and Snacks barriers.

\section{Perceived Self-efficacy Related to Dietary Fat Intake}

Conceptual definition: perception of whether or not an individual perceives him/herself as capable of successfully managing his or her own dietary fat intake (Bandura, 1986; Pender et al., 2002). 
Operational definition: participant's score on the resisting relapse subscale, which is from the Self-efficacy for Eating Behaviors Scale.

\section{Perceived Family Social Support}

Conceptual definition: encouragement a person receives through his/her relationships with family members to practice health-promoting behaviors for healthy eating (Heaney \& Israel, 2008).

Operational definition: participant's total score on the Family Support for Heart Healthy Eating Habits Scale (13 items) was used to determine social support received from family in successfully managing one's fat intake.

\section{Perceived Friend Social Support}

Conceptual definition: encouragement a person receives through his/her relationships with friends to practice health-promoting behaviors for healthy eating (Heaney \& Israel, 2008).

Operational definition: participant's total score on the Friend Support for Heart Healthy Eating Habits Scale (10 items) was used to determine social support received from friends in successfully managing one's fat intake.

\section{Perceived Generalized Stress}

Conceptual definition: the transactions that occur between a person and his/her environment that are taxing or overwhelming to a person (Lyon, 2000).

Operational definition: participant's total score on the Perceived Stress Scale (PSS), a 10-item instrument, was used to determine perceived generalized stress. 


\section{ASSUMPTIONS}

Based on the literature review and the HPM developed by Pender and colleagues (2006), the following assumptions were made:

1. Individuals participating in this study have the ability to examine their own feelings, thoughts, and competencies (reflective self-awareness) related to successful management of dietary fat intake.

2. Individuals value their health potential, which moves them to seek ways to manage their dietary fat intake behaviors.

3. Individuals interact with their environment; thus, their environment changes them and the individuals change their environment over time.

4. Individuals are free to choose their own dietary fat intake behaviors, and they tend to perform behaviors in successful management of their dietary fat intake when they perceive their environment supports them in doing so.

5. Individuals completing the questionnaires are computer literate.

6. Participants complete the questionnaires only once.

7. Participants answer the questionnaires in a truthful manner.

8. Participants answer the questions themselves and do not have someone else complete the questionnaires for them.

9. The key predicting variables are included in this conceptual framework.

\section{LIMITATIONS}

1. Due to convenience sampling, the generalizability of this study is limited. 
2. This study is a cross-sectional design. Therefore, cause and effect relationships cannot be determined.

3. Participants choosing to be in the study may be persons who are interested in dietary fat intake, which may skew the sample.

4. The instruments used to measure each variable are self-report. The participants may not answer truthfully because they want to provide socially desirable responses.

5. Participants may not be able to accurately recall or may not pay attention to their height, weight, and dietary fat intake. Thus, the self-reported data may not be an accurate representation of their actual height, weight, and dietary fat intake.

\section{SUMMARY}

This chapter underscored the prevalence of health problems affecting Black emerging adults (e.g., CVD), which is greatly influenced by their dietary fat intake. The primary purpose of this study was to examine the relationships among individual characteristics (gender, BMI, income adequacy, and religious commitment), behaviorspecific cognitions and affect (perceived barriers for healthy eating, perceived selfefficacy for managing dietary fat intake, family social support, friend social support, and perceived generalized stress), and dietary fat intake. Pender's Health Promotion Model, used to develop this study's conceptual framework, was described, along with the operational definitions of the concepts and variables. The findings from this study offer 
solutions for improving healthy eating habits, particularly successful management of dietary fat intake, among Black emerging adults. 


\section{Chapter 2: Review of the Literature}

This chapter opens with a discussion of Black emerging adults and their dietary fat intake (DFI), dietary fat intake health risks, and cultural food influences that may affect their dietary fat intake. Research examining the relationships between DFI and select predictor variables identified in the HPM (gender, BMI, income adequacy, religious commitment, perceived barriers for healthy eating, perceived self-efficacy related to managing DFI, perceived family social support, perceived friend social support, and perceived generalized stress) is then reviewed.

\section{EMERGING BLACK ADULTS AND DIETARY FAT INTAKE}

The transition from childhood to adulthood in relation to developing healthy dietary habits may be difficult for emerging adults. They are making over 200 food decisions a day (Wansink, 2006; Wansink \& Sobal, 2007) and developing eating behaviors that may be maintained into their elderly years (Hampl \& Betts, 1995; Jensen, 2011). This dissertation study addressed some of the gaps in the literature by focusing on this age group, which may help Black emerging adults develop and maintain healthy eating behaviors into adulthood and their elderly years. Potentially, healthy eating behaviors, which include reducing dietary fat intake, may reduce the risk of chronic lifestyle diseases, especially since CVD often begins during the emerging adult years (Brown et al., 2010; Spencer, 2002).

As reviewed in Chapter 1, the average percentage of total fat intake for adults in the U.S. is 33-34\% (Coulston, Boushey, \& Ferruzzi, 2013). Although these percentages 
are within the recommended limits for total fat intake of $25-35 \%$ (U.S. Department of Agriculture, 2010), researchers have found that most adults and children have excessive fat intake (Kirkpatrick et al., 2012; Wilson, Adolph, \& Butte, 2009). For example, Irazusta et al. (2007) found that first-year university students in Spain had excessive percent energy contributions from fat (females $=45.7 \%$ and males $=45.9 \%$ ).

In addition, total fat intake from 1999 to 2008 increased among Blacks (Wright \& Wang, 2010). Other researchers have also reported high fat-related dietary behaviors, high fat intake, and a preference for high fat foods among Blacks (Beydoun \& Wang, 2008; Brown et al., 2010; Gans, Burkholder, Risica, \& Lasater, 2003; Gary et al., 2004; Hart et al., 2006; Robinson \& Hunter, 2001; Shankar, Dilworth, \& Cone, 2004; Sharma et al., 2009; Zamora et al., 2010). For example, Sharma et al. (2009) reported high consumption of sweetened beverages and high-fat foods in their sample of Black adults, ages $18-74(N=84$, mean age $=45$ years for males and 38 years for females $)$. In a study conducted by Brown and colleagues (2010) among Black college women, they found that the majority of their sample preferred high-fat food (65\%).

Compared to older populations, younger populations, such as emerging adults, frequently consume foods high in fat (Anderson, Winett, Wojcik, \& Williams, 2010; Huang et al., 1994; Spencer, 2002) with a higher percentage of kcal from total fat than the recommended guidelines (Hampl \& Betts, 1995; Kronsberg et al., 2003; Lichtenstein et al., 1998). The frequency of eating fast food, which tends to be high in fat content, has been positively associated with fat intake among samples that included emerging adults (French, Story, Neumark-Sztainer, Fulkerson, \& Hannan, 2001; Jenkins \& Horner, 2005; 
Satia, Galanko, \& Siega-Riz, 2004). The high consumption of fat among Blacks may be in part due to their traditional diet (Gary et al., 2004; Robinson \& Hunter, 2001), which will be reviewed later in this chapter.

Black emerging adults' high consumption of dietary fat intake, particularly saturated fat, may increase their risks for CVD (Brown et al., 2010; Hampl \& Betts, 1995; Hu, Manson, \& Willett, 2001; Kronsberg et al., 2003). The average percentage of saturated fat intake for adults in the U.S. is $11 \%$, which is above the recommendation of $<10 \%$ (Coulston et al., 2013). In a study conducted by Irazusta et al. (2007), first-year university students in Spain had saturated fat intakes above the recommended value (females $=13.4 \%$ and males $=13.3 \%$ ). Saturated fat, also known as "bad fat", can raise blood lipid levels, which can also increase individuals' risk for CVD (AHA, 2011, 2012; Coulston et al., 2013; Dudek, 2010; Temple, Wilson, \& Jacobs, 2012; U.S. Department of Agriculture, 2010; U.S. Department of Health and Human Services, 2013b).

\section{DIETARY FAT INTAKE AND CULTURAL FOOD INFLUENCES}

Black emerging adults' health-promoting behaviors may be influenced by their dietary cultural beliefs. "Food has traditionally been a catalyst for social interaction...for some Blacks, eating is an 'intimate' or a 'spiritual' experience that is shared with others" (Kittler \& Sucher, 2008, p. 220). In general cultural food beliefs have a deep, spiritual connection to the past for Blacks, which dates back to slavery (Braithwaite, Taylor, \& Treadwell, 2009; Dudek, 2010; Hurt, 2013; James, 2004; Kittler \& Sucher, 2008; Liburd, 2003; Luke, Cooper, Prewitt, Adeyemo, \& Forrester, 2001). This connection symbolizes 
love, hospitality, compassion, comfort, happiness, resilience, and collective ethnic identity, to name a few (Bailey, 2006; Hurt, 2013; James, 2004; Kittler \& Sucher, 2008; Liburd, 2003).

One particular cuisine that embodies collective ethnic identity and recognition of Black history is "soul food" (Kittler \& Sucher, 2008). This 400-year-old Black cuisine received its name "because the foods of the ancestors nourish the body, nurture the spirit, and comfort the soul" (James, 2004, p. 361). The term "soul food" was coined in the Southern U.S. in the 1960s (Kittler \& Sucher, 2008; Hurt, 2013). It has also been referred to as "southern food" (Anderson, 2012). "Soul food" is consumed by multiple races/ethnicities, mostly in the South. According to 2013 Chef Survey, 45\% of professional chefs in the U.S. consider "soul food" a perennial favorite (National Restaurant Association, 2012). It is difficult to determine which races/ethnicities eat more "soul food" (Huff \& Kline, 1999). Based on a presentation conducted by the American Heart Association (2013), a study was conducted in the U.S. in which Black and White participants from 48 states answered telephone questionnaires about what type of foods they ate. "About two-thirds of the participants who ate the most Southern-style foods lived in the southeastern United States" (American Heart Association, 2013). To date, there is no documentation of the percentage of Blacks in the U.S. who eat this traditional cuisine, but they are "five times more likely to eat southern foods than Whites" (American Heart Association, 2013).

Some healthy traditional "soul foods" include, but are not limited to, legumes, dark green leafy vegetables (i.e., collard greens, okra, cabbage), yellow vegetables, sweet 
potatoes, and fruit (i.e., watermelon, pumpkin, and tomato) (Bailey, 2006; Braithwaite et al., 2009; Huff \& Kline, 1999; Kittler \& Sucher, 2008; Kulkarni, 2004; Luke et al., 2001), all of which are low in fat. However, food preparation practices, such as frying meats in fat and adding fat as a seasoning for vegetables diminish the healthy aspects of these traditional foods (Bailey, 2006; Bovell-Benjamin, Dawkin, Pace, \& Shikany, 2009; Braithwaite et al., 2009; Huff \& Kline, 1999; Hurt, 2013). Overall, Blacks tend to consume "soul foods" that are high in fat, cholesterol, and sodium (Dudek, 2010; Patterson et al., 1995; Huff \& Kline, 1999; Robinson \& Hunter, 2001) and consume less fruit, vegetables, and fiber than the recommended guidelines (Fitzgibbon et al., 2008). The high consumption of fat among Blacks could be in part due to their traditional diet (Gary et al., 2004; Robinson \& Hunter, 2001).

In addition to historical cultural factors such as slavery and "soul food", another cultural influence related to food is family heritage (James, 2004). Food is an integral part of many extended Black family gatherings and traditions (James, 2004; Kittler \& Sucher, 2008; Liburd, 2003). Among most Black families eating is a "ritual" that communicates love towards other family members (Baskin, Odoms-Young, Kumanyika, \& Ard, 2009). In general, Black families take pride in passing on how to cook traditional foods; it is a tradition they hope to pass on to future generations (James, 2004, Jenkins, 2007; Peters, Aroian, \& Flack, 2006; Kulkarni, 2004; Shankar et al., 2004). Cultural traditions have a profound influence on individuals' food choices (Dacosta \& Wilson, 1996; McGee, et al., 2014; Peters et al., 2006; Shankar et al., 2004), including Black emerging adults (Shankar et al., 2004). Holding on to this heritage is important as described in the following 
manner, "I know that some of our traditional foods are not full of nutrients but they bring back good memories of childhood and I'm not giving them up just because some researcher says they are bad" (James, 2004, p. 358).

For some, not keeping such traditions may mean giving up part of one's cultural heritage and trying to conform to the dominant culture (James, 2004; Peters et al., 2006), which may be perceived as harmful to their identity (Baskin et al., 2009). Individuals, who are willing to make healthy food choice changes despite their cultural heritage, find that their family members and friends are not usually supportive of dietary changes that break cultural traditions (James, 2004; Peters et al., 2006). "The need to prevent alienation from one's culture would lead a person to avoid new behaviors patterns that are culturally unacceptable" (Kumanyika, Morssink, \& Agurs, 1992, pp. 169-170). While this literature review highlights the importance of cultural influences related to food such as the upholding of one's cultural heritage, researchers need to be sensitive to the great diversity within Black cultures. However, it is important to consider cultural influences related to food, particularly fat intake, for the context of this current study.

\section{FACTORS IMPACTING DIETARY FAT INTAKE}

Using the Health Promotion Model as a theoretical framework, the next sections examine research on factors that may influence dietary fat intake among Black emerging adults: individual characteristics (gender, BMI, income adequacy, and religious commitment) and behavior-specific cognitions and affect (perceived barriers for healthy eating, perceived self-efficacy related to managing dietary fat intake, perceived family 
social support, perceived friend social support, and perceived generalized stress). Research studies are reviewed regarding the potential moderating effects of perceived family and friend social support and religious commitment. Lastly, research studies are reviewed regarding the potential mediating effect of religious commitment.

\section{Gender and DFI}

As described in Chapter 1, gender is one of the individual characteristics that may influence health-promoting behaviors. Gender differences have been noted in the literature; females are more likely to eat healthier, to comply with dietary guidelines, and to be knowledgeable about nutrition as compared to males (Baker \& Wardle, 2003; Hiza, Casavale, Guenther, \& Davis, 2013; Hsiao et al., 2013; Morse \& Driskell, 2009; Nayga, 2000; Sijtsma et al., 2012; Turrell, 1997). They also tend to consume less fat compared to males (Beydoun \& Wang, 2008; Brug, Van Assema, Kok, Lenderink, \& Glanz, 1994; Cartwright et al., 2003; Fernandes et al., 2013; Feunekes et al., 1998; Hermstad et al., 2010; Harcrow, 2010; Hart et al., 2006; Huang et al., 1994; Jen et al., 2007; Kennedy et al., 1999; Kim \& Sobal, 2004; Watters \& Satia, 2009). Along with consuming more dietary fat intake, male college students less frequently limit their dietary fat intake (Berg et al., 2013).

Cartwright and colleagues (2003) found that teenage females had lower odds of consuming high-fat foods $(\mathrm{OR}=.56[.49-.64], p=.00)$ compared to males. Spencer (2002) found that females were less likely to eat diets high in saturated fats compared to males $\left(\chi^{2}=25.01, d f=3, p=.00\right)$. Harcrow (2010) found that males had significantly higher fat intake scores $(M=30.44, S D=10.19, F=10.71 ; p=.001)$ compared to 
females $(M=28.22, S D=8.95)$. In a study conducted by Hart et al. (2006), fat summary scores among males indicated higher fat intake $(n=329$, fat score $=2.57, S D=.44, p<$ $.001)$ compared to females $(n=2,030$, fat score $=2.44, S D=.41)$. Similarly, Jen et al. (2007) found that males' total fat (64.5 grams) and saturated fat (20.3 grams) intake was significantly higher than females' total fat (49.6 grams) and saturated fat (16 grams) ( $p<$ $.001)$ intake.

There are mixed findings regarding whether or not gender is a significant predictor for dietary fat intake. Grizzle (2009) found gender not to be a significant predictor of fat-related habits in a study among police officers. However, Harcrow (2010) found gender to be a significant predictor for DFI among a sample of college students ( $B$ $=-2.37, p=.001)$.

Although the gender difference in fat consumption is consistent in the literature with males having higher fat intake compared to females, more research is needed to examine the gender difference among emerging adults. The samples in the studies reviewed above had an age range between 15 and 91 years. Researchers of five of the above studies reported inclusion of emerging adults in their samples (Harcrow, 2010; Huang et al., 1994; Kennedy et al., 1999; Spencer, 2002; Watters \& Satia, 2009). Cartwright et al. (2003) (teenagers living in London) and Feunekes et al. (1998) (15 year olds living in The Netherlands) had samples that included younger individuals. However, these individuals lived outside of the United States. Only two of the studies focused on emerging adult populations (Harcrow, 2010; Huang et al., 1994). This current dissertation 
study addresses the considerable gap of examining gender differences for dietary fat intake in regards to a specific age group, emerging adults.

Ethnicity was not reported in some of the studies, which makes it difficult to determine if Blacks were underrepresented in these samples. Only two of the reviewed studies above had all Black samples (Jen et al., 2007; Watters \& Satia, 2009), and Hermstad et al. (2010) reported $49.5 \%$ of their sample as Black. The findings from this dissertation study may help researchers develop culturally, age-appropriate, and genderspecific interventions to help Black emerging adults decrease their dietary fat intake.

\section{Body Mass Index and DFI}

As described in Chapter 1, BMI is the one of the individual characteristics that in general has a positive association with unhealthy dietary behaviors. For example, adherence to a healthy diet, which included reduced-fat dairy foods and low amount of fast food, was positively associated with lower BMI and smaller gains in BMI (Miller et al., 2012; Newby et al., 2003). Specifically, the literature on BMI's relationship with dietary fat intake has been mixed. For example, although it was not statistically significant, Vadiveloo, Scott, Quatromoni, Jacques, and Parekh (2014) reported increases in the percentage of fat consumed over time (1991-2008) among participants in all BMI categories. The percentage of calories from fat consumed increases were the following: normal weight fat $=26.6 \%$ to $28.9 \%$, overweight $=26.4 \%$ to $29.7 \%$, and obese $=28.5 \%$ to $30.9 \%$. Omondi, Othuon, and Mbagaya (2011) reported a small positive correlation between BMI and fat intake, but it was not statistically significant. Howarth, Huang, Roberts, and McCrory (2005) found no significant relationship between BMI and fat 
intake among females; however, they found a significant relationship among males $(B=$ $.09, p=.003$ ). However, Cartwright and colleagues (2003) found that overweight children had less odds of consuming more high-fat foods ( $\mathrm{OR}=.73[.65-.83$ ], $p=.00$ ) compared to normal weight children. In contrast, Ledikwe et al. (2003) found positive relationships between BMI and fat intake $(r=0.26)$ and BMI and saturated fat $(r=0.21)$ among females $(p<.05)$ and not among males. Similarly, in a literature review conducted by Bray and Popkin (1998), they ran an ordinary least-squares regression for a sample of 20 countries, with high overweight populations (BMI >25), to examine the relationship between BMI and proportion of dietary energy from fat. The researchers found a positive relationship between the proportion of dietary energy from fat and BMI $(\beta=2.53, p<$ $\left..001, R^{2}=.78\right)$. There was an increase in BMI in countries that consumed high amounts of dietary fat (Bray \& Popkin, 1998). Blundell and Macdiarmid (1997) found 19 times more participants with BMI's $>30$ in their high-fat group compared to the low-fat group. In a review of the literature conducted by the National Heart Foundation of Australia (2003), they reported inconsistent significant relationships between BMI and dietary fat intake, with correlation coefficients ranging from 0.17 to 0.38 .

Although the reviewed literature reveals mixed findings regarding the relationship between BMI and fat intake among human populations, earlier researchers found a statistically significant positive relationship in studies with rats and mice (Boozer, Schoenbach, \& Atkinson, 1995; Salmon \& Flatt, 1985; Schemmel, Mickelsen, \& Motawi, 1972). The lack of consistent, statistically significant relationships between BMI and fat intake among human populations may be due to measurement error in dietary 
data, underreporting of fat intake, and factors such as age, gender, genetics, metabolism, rate of fat oxidation, and level of physical activity (Blundell \& Macdiarmid, 1997; Bray \& Popkin, 1998; Omondi et al., 2011; National Heart Foundation of Australia, 2003). Further, carbohydrates and proteins contain $4 \mathrm{kcal} / \mathrm{gram}$ and fats contain $9 \mathrm{kcal} / \mathrm{gram}$ (Dudek, 2014). Logically, individuals with higher fat intake are likely to have a high-kcal diet; but low dietary fat intake is not always indicative of a low-kcal diet since individuals may not decrease their total energy intake (calories consumed). Also, some low-fat food products are not necessarily low calorie.

A majority of the studies reviewed above are among adult populations, with $M$ age $\geq 38$. Only one of the above studies reported the inclusion of emerging adults, sample ranged from age 20 to 59 years (Howarth et al., 2005). Further, ethnicity was not reported in some of the studies, which makes it difficult to determine if Blacks were underrepresented in their samples. This current dissertation study may help to shed light on the inconsistent reports of the relationship between BMI and dietary fat intake. In addition, it addressed the substantial gap in the literature in regards to age and ethnicity/race since there are few BMI and dietary fat intake studies among emerging Black adults. Using the HPM as a theoretical framework as discussed in Chapter 1, this study's examination of BMI and fat intake among Black emerging adults will help address these gaps and inconsistencies in the literature.

\section{Income Adequacy and DFI}

As reviewed in Chapter 1, income adequacy is one of the individual characteristics. Having an adequate income can be a determinant of individuals' 
consumption of healthy diets (Beydoun, Powell, \& Wang, 2008; Brug, 2008; Darmon \& Drewnowski, 2008; Hiza et al., 2013; Inglis et al., 2009; Kirkpatrick et al., 2012; Raffensperger et al., 2010). If individuals perceive themselves as having adequate income, then they may spend their money on healthy food. If they do not perceive themselves as having adequate income, then they may spend their money on food that tends to cost less and be high in fat (Drewnowski \& Darmon, 2005; Drewnowski \& Specter, 2004). There are various methods to assess income (e.g., socioeconomic status and annual income). Therefore, for the purpose of this current literature review, studies pertaining to income adequacy, income, and/or socioeconomic status have been included. The term income adequacy is used when describing these studies.

Both non-significant and significant findings have been reported in regards to the relationship between income adequacy and dietary fat intake. Deshmuk-Taskar et al. (2007), Hart et al. (2006), and Jen et al. (2007) found no significant relationship between income and dietary fat intake. When Hart et al. (2006) adjusted for age, there was a significant relationship with fat intake $(p=.001)$; however when they included education in the model the relationship was no longer statistically significant $(p=.18)$. Beydoun and Wang (2008) found a positive relationship between income and better diet quality in relationship to fat intake, reduced fat consumption. Researchers suggest that healthier diets cost more (Drewnowski \& Darmon, 2005; Drewnowski \& Specter, 2004), which may not be affordable for low-income families. Therefore, low-income families may increase their consumption of grains, added sugars, and fats to keep dietary costs down (Drewnowski \& Darmon, 2005; Drewnowski \& Specter, 2004). 
In a qualitative study conducted by DiSantis et al. (2013) among Black adults and youths, they found similar results in regards to purchasing low-priced, high-fat content foods. Participants described the importance of stretching their food money by purchasing low-priced foods (DiSantis et al., 2013), which tend to be high in fat (Drewnowski \& Specter, 2004). The youth reported choosing low-priced foods and stated, "We just kind of like cheaper stuff, like fast food, because it is cheaper" (DiSantis et al., 2013, p. 517). Adult participants also described moments when they purchased low-priced unhealthy foods because they could not afford the healthy foods. In contrast, some participants discussed that healthy foods were more important than seeking lowpriced foods. However, they "expressed frustration at having to pay a higher price for healthier options," such as stating, "It is more expensive, but sometimes you have to go that extra mile" (DiSantis et al., 2013, p. 518).

Researchers have shown that protein suppresses energy intake greater than fat and carbohydrates (Blundell \& Stubbs, 2004). They have also reported that high-fat foods inhibit the body's satiety signals, which can lead to overeating and high caloric intake (Blundell et al., 1993; Blundell \& Stubbs, 2004; Jebb \& Prentice, 2001). Blundell et al. (1993) reported, "fat has a weak effect on satiation" (p. 776S). Jebb and Prentice (2001) suggest "dietary fat is associated with passive over-consumption as a consequence of its relatively high energy density and weak effects on both satiation and satiety" (p. 133). These findings may explain increases in obesity prevalence and dietary fat intake among Black emerging adults since individuals may purchase more low-priced, high-fat content foods in order to stretch their money, as previously described. 
Emerging adults face unique financial challenges as they transition into becoming independent adults, such as increased expenses and decreased employment (Pew Research Center, 2013a). In 2012 according to the Pew Research Center (2013a), 36\% of young adults, ages 18 to 31 years, lived in their parents' home. Since the onset of the Great Recession 2007-2009, individuals who are ages 18 to 24 years (56\%) are more likely to be living with their parents compared to older young adults (16\%) (Pew Research Center, 2013a). Staying with their parents may help emerging adults save on living expenses (e.g., rent and utilities) and pay their debt (e.g., college loans), particularly when they are struggling to find employment (U.S. Congress Joint Economic Committee, 2010). The unemployment rate for young workers reached a record $19.6 \%$ in April 2010, with Black young workers having the highest rates (U.S. Congress Joint Economic Committee, 2010). It is possible for emerging adults living on their own and those depending on their parents/guardians for support to perceive their income as inadequate to eat healthy.

Only two of the studies reviewed above had samples that had a majority of Black participants (DiSantis et al., 2013; Jen et al., 2007). Two other studies had samples that included youth and emerging adults (Beydoun \& Wang, 2008; Deshmuk-Taskar et al., 2007). This current dissertation study focuses on Black emerging adults, which may help address the age-gap in the literature. Examination of the relationship between income adequacy and fat intake in this current study may shed light on the mixed findings reported in the studies reviewed above, which focused on income adequacy, income, and/or socioeconomic status. Using the HPM as a theoretical framework as discussed in 
Chapter 1, the findings from this study's examination of income adequacy and dietary may help policy makers and researchers develop food programs that will help emerging adults decrease their dietary fat intake.

\section{Religious Commitment and DFI}

As reviewed in Chapter 1, religious commitment is one of the individual characteristics. The church and religion often play a major role among Black populations, motivating social action and providing social and economic resources (Campbell et al., 2007; Pattillo-McCoy, 1998), which is one reason why religious commitment is examined in this proposed study.

Religious commitment describes the "degree of internal commitment a person feels toward his religious beliefs. It also reflects the influence that religious beliefs and teachings have on the person's decisions and lifestyle" (Koenig et al., 2001, p. 500). Koenig et al. (2001) reported that intrinsic religiosity is one of the best indicators of religious commitment. In 1967 Allport and Ross (as cited in Koenig et al., 2001) reported that religion is the principal motivation of behavior in an intrinsically religious individual. Allport and Ross also reported that intrinsically religious individuals have "embraced a creed" and "endeavors to internalize it and follow it fully" (as cited in Koenig et al., 2001, p. 21). Therefore, for the purposes of this current study, religious commitment is defined as the degree of internal commitment to one's religious beliefs, which in turn should motivate an individual's behavior (e.g., manage dietary fat intake) (Koenig et al., 2001). 
As described in Chapter 1, religious commitment is classified as a sociocultural factor. There are many individual cultural beliefs and practices pertaining to religious commitment that may influence one's health behaviors. Researchers may sometimes use religious terms interchangeably (e.g., religion, religiosity, spirituality, and religious commitment) (Cohen \& Koenig, 2003; Harcrow, 2010; Hernandez, 2011; Hyman \& Handal, 2006; Joshi, Hardy, \& Hawkins, 2009; Koenig, 2012; Musick, Traphagan, Koenig, \& Larson, 2000; Roberts \& Yamane, 2003; Russinova \& Cash, 2007; Takamizawa, 1999; Tan, Chan, \& Reidpath, 2013; Vachon, Fillion, \& Achille, 2009; Waldron-Perrine et al., 2011; Watson \& Nesti, 2005). The practice of interchanging religious terms makes it a challenge to assess which studies truly pertain to "religious commitment." Therefore, for this current literature review on religious commitment, studies pertaining to religion, religiosity, spirituality, and/or religious commitment have been included.

In general there is a positive relationship between religion, religiosity, spirituality, and/or religious commitment and health; religious individuals tend to practice healthy behaviors, including those who are younger in age (e.g., self-management of diabetes, use seat belts, exercise, and have lower rates of smoking, alcohol and drug use, and premarital sex) (Callaghan, 2006; Coruh, Ayele, Pugh, \& Mulligan, 2005; Ellison \& Hummer, 2010; Ellison \& Levin, 1998; Gillum, King, Obisesan, \& Koenig, 2008; Hill, Ellison, Burdette, \& Musick, 2006; Jones, 2012; Joshi et al., 2009; Koenig, 2012; Koenig et al., 2001; Larson \& Larson, 2003; McDougle, Handy, Konrath, \& Walk, 2013; McNamara, Burns, Johnson, \& McCorkle, 2010; Obisesan, Livingston, Trulear, \& 
Gillum, 2006; Park, Edmondson, Hale-Smith, \& Blank, 2009; Piderman, Schneekloth, Pankratz, Maloney, \& Altchuler, 2007; Polzer \& Miles, 2007; Powell, Shahabi, \& Thoresen, 2003, Rew \& Wong, 2006; Rew, Wong, Torres, Howell, 2007; Schlundt et al., 2008; Tan et al., 2013). Religion, religiosity, spirituality, and/or religious commitment appear to be protective factors for physical and psychological morbidity among Blacks and all younger populations of any ethnicity (Cotton, Zebracki, Rosenthal, Tsevat, \& Drotar, 2006; Joshi et al., 2009; Koenig et al., 2001; Levin, Chatters, \& Taylor, 2005; McNamara et al., 2010).

There were similar findings regarding the relationships between religion, religiosity, spirituality, and/or religious commitment and nutrition, and various predictors for nutritional intake (Merrill \& Thygerson, 2001; Pearson, Turner, Young, \& Knickerbocker, 2007; Strawbridge, Shema, Cohen, \& Kaplan, 2001). In a literature review conducted by Koenig (2012), he found that religious individuals tend to consume healthy diets. Researchers have reported a positive relationship between healthy eating and religion, religiosity, spirituality, and/or religious commitment ( $r=.02$ to .428 ) (Chester et al., 2006; Rew, Arheart, Thompson, \& Johnson, 2013; Schlundt et al., 2008). In addition, Reeves et al. (2012) found that individuals who prayed more reported consuming fewer calories per day, and Callaghan (2006) found that those who routinely practiced their religion had higher nutrition scores compared to those who did not ( $t=$ $3.61, p=.00)$.

In relation to dietary fat intake, there are equivocal findings in the literature about the relationships between religion, religiosity, spirituality, and/or religious commitment 
and dietary fat intake. For example, Schlundt et al. (2008) found a small, positive relationship between fat intake and religious variables $(r=.10, p<.0001)$. Conversely, Fitzgibbon et al. (2005), Harcrow (2010), and Reeves et al. (2012) did not find any statistically significant relationships between religion, religiosity, spirituality, and/or religious commitment and dietary fat intake. These same equivocal findings regarding the relationship between religion variables and dietary fat intake were found in a literature review conducted by Tan et al. (2013). They found among 27 studies: no significant relationship for 15 studies (55.6\%), negative relationship for seven studies $(25.9 \%)$, positive relationship for three studies (11.1\%), and mixed findings for two studies $(7.4 \%)$ (Tan et al., 2013).

Of the reviewed studies above that reported ethnicity/race, two of the studies had an adequate representation of Black participants: 1) all Black women $(N=59)$ (Fitzgibbon et al., 2005) and 2) 63\% Blacks $(n=1905)$ (Schlundt et al., 2008). Studies reviewed by Tan et al. (2013) had emerging adult samples, and Harcrow (2010) also reported having emerging adults. However, the findings for individuals $\leq 25$ years is not teased out from most of the reported data.

Unlike the reviewed studies above, this current dissertation study focuses on Black emerging adults and their religious commitment in relation to dietary fat intake. Using the HPM as a theoretical framework as discussed in Chapter 1, the findings from this study can help to clarify the inconsistent relationship findings in the literature by using a consistent term to describe an individual's internal commitment to his/her religious beliefs, religious commitment. 


\section{Perceived Barriers for Healthy Eating and DFI}

As reviewed in Chapter 1, perceived barriers is one of the behavior-specific cognitions and affect factors. When adults encounter significant barriers in trying to eat healthy, they may not be successful in their attempt to manage their fat intake. Being too busy and lack of time have been reported as primary barriers for eating healthy (DiSantis et al., 2013; Hargreaves et al., 2002; Lucan, Barag, Karasz, Palmer, \& Long, 2012; Sigrist, Anderson, \& Auld, 2005; Welsh et al., 2012). Other barriers reported among adult populations in the literature include lack of knowledge (e.g., not sure what to eat), lack of social support, sense of social isolation, competing family responsibilities, motivation, lack of control, non-availability of healthy food options, transportation, cost, dislike of cooking, food preferences, social and cultural symbolism of certain foods, taste, poor oral health, confusing messages from the media/research, lack of cooking skills, and eating healthy not being a priority (Abbott, Davison, Moore, \& Rubinstein, 2010; AbuSabha \& Achterberg, 1997; Brug, 2008; Calvert \& Isaac-Savage, 2013; DiSantis et al., 2013; Hargreaves et al., 2002; James, 2004; Kumanyika, 2007; Lucan et al., 2012; Nicklas et al., 2013; Pawlak \& Colby, 2009; Sigrist et al., 2005; Timmerman, 2007; Walcott-McQuigg, 1995; Welsh et al., 2012).

Similar to adult populations, younger populations encounter barriers to practicing healthy behaviors (e.g., eating healthy) (Fila \& Smith, 2006; Horton, 2013a; Jenkins \& Horner, 2005). Younger populations have identified not having enough time to eat as a barrier in trying to eat healthy (French et al., 2001; Horton, 2013a; Jenkins \& Horner, 2005; Neumark-Sztainer et al., 1999). To save on time and meal planning, young adults 
and teenagers ate at restaurants and from vending machines (e.g., junk food) (Jenkins \& Horner, 2005; Neumark-Sztainer et al., 1999). Other barriers for younger populations include availability and taste of foods, food cravings, cost, little care about eating healthy, and media (e.g., fast food commercials) (Fila \& Smith, 2006; French et al., 2001; Jenkins, 2007; Neumark-Sztainer et al., 1999).

These barriers for healthy eating are not different from the barriers identified for eating fewer high-fat foods among both young and older populations: lack of time, not being a priority, cost, inconvenience, taste quality of food, taste preferences for other foods, lack of family support, inability to evaluate fat content of foods, and difficulty finding healthy alternatives when eating out (e.g., low-fat options on the menu) (AbuSabha \& Achterberg, 1997; Beydoun \& Wang, 2008; Feunekes et al., 1998; Koikkalainen et al., 1999; Lloyd et al., 1995; Neumark-Sztainer et al., 1999). For example, Lloyd and colleagues' (1995) experimental study among United Kingdom participants (ages 18 to 55 years, $M=35.1$ ) used discriminant analysis to identify the main factors (barriers) for more successful and less successful groups in reducing their fat intake. The researchers found that liking the change of increasing one's intake of reduced fat products $($ factor coefficient $=-.80, p<.01)$ and taste $($ factor coefficient $=.57, p<.01)$ were significant predictors for increasing one's intake of reduced fat products, while liking the change of reducing intake of red meat $(-.40, p<.05)$ and convenience $(-.37, p<$ .05) were significant predictors for reducing one's intake of red meat.

Koikkalainen et al. (1999) found that eating in social settings can also serve as a barrier in eating foods low in fat. Using $\chi^{2}$ tests, the researchers found that participants 
with high fat intake reported eating like other people without thinking about what they are eating as more of a barrier compared to participants with low fat intake ( $p=.02$ to .004). Participants ate like others around them so as not to be different (Koikkalainen et al., 1999).

Beydoun and Wang (2008) found that the perceived barrier of food price was a mediator between socio-economic status and selected diet quality indicators, to include fat intake (mediation proportion $=10 \%$ ). Watters and Satia $(2009)$ examined perceived barriers to healthy eating, which they defined as enabling factors (feel you can afford healthy foods, time/trouble to prepare healthy foods, it is easy to order healthy foods at restaurants, and need information to prepare healthy foods). Eleven percent (Adjusted $\mathrm{R}^{2}$ ) of the variance in total fat intake (grams/day) and $12 \%$ (Adjusted $\mathrm{R}^{2}$ ) of the variance in saturated fat intake was due to perceived barriers to healthy eating (enabling factors). Feunekes and colleagues' (1998) qualitative portion of their study and Neumark-Sztainer and colleagues' (1999) qualitative study support the above barrier findings and provide additional insight to how barriers influence food choices.

More research is needed regarding barriers for individuals ages 18 to 25 years since the majority of the reviewed literature focuses on adults, adolescents, and children. Although French et al. (2001) and Neumark-Sztainer et al. (1999) examined barriers among adolescents, little focus has been placed on emerging adults. There are some studies reviewed above in which the sample age range captured emerging adults: Abbott et al. (2010) ages 19 to 72 years $(M=48)$; Beydoun and Wang (2008) ages 20 to 65 years $(M=40)$ and Blacks $=12 \%$; Lloyd et al. $(1995)$ ages 18 to 55 years $(M=35.1)$; and 
Walcott-McQuigg (1995) Black females, ages 25 to 75 years $(M=40)$. However, the mean age of these samples is above 25 years. In addition, there is no documentation of ethnicity in some of the studies, which makes it difficult to determine if Blacks were underrepresented in their samples.

Using the HPM as a theoretical framework, perceived barriers for healthy eating may directly influence the health behavior of dietary fat intake in that as barriers increase then the likelihood of individuals successfully managing their dietary fat intake decreases. This dissertation study's examination of perceived barriers among Black emerging adults addresses this age-gap in the literature. The findings may help researchers develop culturally sensitive interventions to help this population manage/overcome identified barriers.

\section{Perceived Self-Efficacy and DFI}

As described in Chapter 1, perceived self-efficacy is one of the behavior-specific cognitions and affect factors. Perceived self-efficacy is an individual's perception of whether or not he/she is capable of performing a particular task (i.e., successfully managing dietary fat intake) (Bandura, 1986; Pender et al., 2002). If individuals perceive themselves capable of successfully managing their dietary fat intake, then they are more likely to consume less dietary fat. Self-efficacy has been found to be a strong predictor of healthy nutrition-related behaviors (AbuSabha \& Achterberg, 1997; Clark \& Dodge, 1999; Delahanty, Hayden, Meigs, Nathan, \& Williamson, 2002), with individuals who have higher self-efficacy practicing healthier eating behaviors. 
AbuSbha and Achterberg (1997) conducted a literature review of 37 studies. They examined self-efficacy and adherence to weight-loss programs. Twelve of the 37 studies pertained to self-efficacy and food-related behaviors. They reported correlation coefficients ranging from -.01 to -.23 . In addition, they found that self-efficacy explained $28 \%$ to $66 \%$ of the variation in the nutrition outcomes.

Researchers have reported similar findings examining the relationship between self-efficacy and dietary fat intake (Anderson-Bill, Winett, \& Wojcik, 2011; Armitage \& Conner, 1999; Chang et al., 2008; Grembowski et al., 1993; Povey et al., 2000; Scholz, Ochsner, Hornung, \& Knoll, 2013; Schwarzer \& Renner, 2000; Smith \& Owen, 1992; Watters \& Satia, 2009). Significant negative correlations between self-efficacy and fat intake ranged from -.18 to -.37 (Anderson-Bill et al., 2011; Armitage \& Conner, 1999; Povey et al., 2000). Watters and Satia (2009) found that participants with high selfefficacy consumed less total fat $(M=29.3$ grams/day, $p=.03)$ and saturated fat $(M=11.1$ grams/day, $p=.04)$ compared to those who had low self-efficacy $(M=35.4$ grams/day and 13.4 grams/day, respectively). Brug et al. (1994) found that Dutch participants who reported difficulty in refusing high-fat foods had high dietary fat intake $(p<.01)$. Smith and Owen (1992) found that participants with greater self-efficacy to eat healthy in social situations $($ dietary fat intake $=33.9 \%$, percent variance $=1.7 \%, F[3,366]=3.2, p<.05)$ had lower dietary fat intake compared to those with low self-efficacy in social situations (dietary fat intake $=36.1 \%$ ). Based upon the reviewed literature, in general individuals with high self-efficacy consume less fat. 
In one study, self-efficacy was a significant predictor for one's intention to eat a low-fat diet $(B=-1.31, S E B=.46$, Final $\beta=-.35, p<.001)$, but not a significant predictor for actual fat intake (Armitage \& Conner, 1999). Povey et al. (2000) found a positive relationship between self-efficacy and one's intention to eat a low-fat diet ( $\beta=$ $.291, p<.001)$. For each unit increase in self-efficacy there was a .291 unit increase in one's intention to eat a low-fat diet. There was a negative relationship between selfefficacy and fat intake $(\beta=-.262, p<.05)$. For each unit increase in self-efficacy there was a .262 unit decrease in one's fat intake.

More research is needed regarding self-efficacy and fat intake among emerging adults. The age range among the studies' samples reviewed was between 16 to 81 years. The reported mean ages for the above studies ranged from 23 to 52.34 years, with most of them having a mean age $\geq 28$. Only Armitage and Conner's study (1999) had a mean age that captured emerging adults $(M=23)$, which was conducted in the United Kingdom. Only two studies reviewed had adequate representation of in Blacks in the sample: 1) 49.9\% Black women $(n=200)$ (Chang et al., 2008) and 2) all Blacks $(n=1905)$ (Watters \& Satia, 2009). In addition, although the reviewed studies were conducted between the years 1992 and 2013, only four of these studies were conducted within the last five years. The lack of current research on self-efficacy and fat intake among Black emerging adults is a substantial gap in the literature.

Based on the reviewed literature, individuals with high self-efficacy consume less dietary fat. In Pender and colleagues' (2002) revised HPM, perceived self-efficacy directly and indirectly (through commitment to a plan of action) influenced the behavior 
outcome. For this dissertation study, perceived self-efficacy related to dietary fat intake may directly influence dietary fat intake in that as self-efficacy increases then the likelihood of individuals successfully managing their dietary fat intake also increases. The findings from this current study addresses the age-gap in the literature and may help researchers develop culturally sensitive interventions to help Black emerging adults increase their self-efficacy in successfully managing with dietary fat intake.

\section{Perceived Social Support and DFI}

As described in Chapter 1, social support is one of the behavior-specific cognitions and affect factors. Social support is the encouragement an individual receives through his/her relationships with others. Most the literature does not distinctly separate social support into family and friends; therefore, it will be examined as one entity in this section. In general there is a positive relationship between social support and individuals' practicing health-promoting behaviors (e.g., eat healthy), and social support tends to be a significant predictor of healthy eating behaviors (Aggarwal, Liao, Allegrante, \& Mosca, 2010; Debnam, Holt, Clark, Roth, \& Southward, 2012; Fowles et al., 2012; Kelsey et al., 1996). For example, Kelsey et al. (1996) conducted multiple regression analyses and found that among women support from friends was a significant predictor for healthy dietary changes $(F[1,181]=4.91, p<.05)$. The researchers did not report $R^{2}$ or betas for the multiple regression analyses. Debnam et al. (2012) and Fowles et al. (2012) found a positive relationship between healthy diets and social support, with correlations ranging up to .38. Aggarwal et al. (2010) found that participants with low social support were 2.7 times as likely not to adhere to a healthy diet compared to those with high social support. 
When reviewing the relationship between social support and dietary fat intake there are mixed findings reported in the literature. Some researchers have not found a statistically significant relationship between social support and dietary fat intake (Anderson-Bill et al., 2011; Harcrow, 2010; Hermstad et al., 2010; Kim \& Sobal, 2004; Thrasher et al., 2004). It is possible that social support was not significant in that there are multiple social, cultural, and environmental factors, which envelop social support, influencing dietary fat intake (Hermstad et al., 2010; Brug, 2008).

However, Scholz et al. (2013) found a significant positive relationship between dietary low fat intake and social support $(p<.01)$ : emotional social support $(r=.20)$ or being encouraged when one encounters problems, and instrumental social support ( $r=$ .25 ) or the practical help one receives from another person. As social support increased, low dietary fat intake increased. Their findings showed that instrumental social support predicted low dietary fat intake $(\beta=.26, p<.01)$. Similarly, Rees, Karter, and Young (2010) found a significant positive association between social support and controlling calories and/or fat intake, particularly for Blacks. A 1-point increase in social support was associated with an $84 \%$ increase in the odds of controlling calories and/or fat intake among Blacks (95\% CI: 1.05, 3.22) (Rees et al., 2010). Anderson-Bill et al. (2011) found that social support received from family and friends contributed to lower levels of fat intake $\left(\right.$ beta $\left._{\text {total }}=-.28, p<.001\right)$.

Peters et al. (2006) found in their qualitative study, five focus groups of Black participants ( $n=34$, ages 27 to 60 years), that Blacks believed they had a responsibility to offer others, within their culture, support in living healthier lives. However, the 
researchers also found a lack of support among participants who were trying to live healthier lives (e.g., eat healthy). Examples of lack of support included calling the participants names, such as "diet Nazi," and family members refusing to come to participants' homes because they do not like the healthy foods served or bring their own food (Peters et al., 2006).

In contrast, some researchers have found a positive relationship between social support and fat intake, in that as social support increases so does one's fat intake (Brug, et al. 1994; Harcrow, 2010; Kim, McIntosh, Kubena, \& Sobal, 2008; Watters \& Satia, 2009). Brug and colleagues (1994) examined the relationship between social influence (encouragement to eat a low-fat diet) and fat intake. Although small, they found a significant positive relationship between encouragement to eat a low-fat diet and fat intake $(r=.06, p<.05)$.

Similarly, Watters and Satia (2009) examined social support, which they defined as reinforcing factors (encourage you to eat healthy foods, tell you about healthier foods, prepare healthier foods with you, and eat healthier food with you). Ten percent (Adjusted $\mathrm{R}^{2}$ ) of the variance in total fat intake (grams/day) and $12 \%$ (Adjusted $\mathrm{R}^{2}$ ) of the variance in saturated fat intake was due to social support. Participants, who felt they could count on those close to them a lot to help them eat healthier, consumed more total fat $(M=33.9$ grams/day, $p=.03)$ and saturated fat $(M=12.9$ grams/day, $p=.02)$ compared to those who reported they could count on those close to them not at all ( $M=33$ grams/day and $M$ = 12 grams/day, respectively) (Watters \& Satia, 2009).

Watters and Satia (2009) mention that it is possible that the measured social 
support variables in their study were not salient in their sample of Blacks, ages 18 to 70 years. The above positive relationship results for social support and dietary fat intake may be due how social support was measured: global social support versus specific social support for healthy eating or decreased fat intake. Further, the results may be linked to the barriers previously described, such as individuals' report of eating like other people around them without thinking about what they are eating. For example, in Hargreaves and colleagues' (2002) qualitative research study, participants commented on how their friends influenced their food choices: "My co-workers influence me to eat foods that are high in fat" (p. 140). There may be pressure from family and friends to eat unhealthy foods. James (2004) and Peters et al. (2006) described how individuals might feel isolated when they attempt to eat healthier since family members and friend may not be supportive of these dietary changes. Therefore, individuals may continue to eat unhealthy foods (e.g., consume foods high in fat) in order to continue their acceptance by their family and friends.

In contrast, some researchers have found a negative relationship between social support and fat intake (Bull, Eakin, Reeves, \& Kimberly, 2006; Hart et al., 2007; Kim et al., 2008). For example, Bull et al. (2006) found that having greater multi-level support was associated with better dietary behavior scores, lower scores indicate better dietary fat and fiber intake behaviors, with $r=-.34$ for all levels of support $(p<.001)$ and $r=-.299$ for family and friends support. Within religious settings, Hart et al. (2007) found that lower fat intake was significantly associated with leadership support scores $(p=.024)$. Individuals who received high support from their leaders had low fat intake. Using 
discriminant analysis, Lloyd et al. (1995) found similar findings in that family support was a significant predictor for reducing total fat intake $(b=.65, p<.01)$.

Based on the reviewed literature, there are mixed findings regarding the relationship between social support and dietary fat intake. Perhaps the mixed findings are due to how researchers measured and/or defined social support. For this study, the measurement of social support was specifically chosen to assess the social support individuals receive for eating a heart healthy diet, which includes low fat eating behaviors, rather than general social support. In addition, the literature review reveals gaps in the literature regarding the age and ethnic/race characteristics among samples. Most of the literature reviewed in this section focused on older adults. The reviewed studies' sample age ranged from 17 to 87 years, but findings specific to emerging adults were not reported. The study conducted by Hart et al. (2007) is the only one that attempts to provide demographic data for its younger individuals (ages 18 to 29 years $=3.6 \%$ ) and analyze the data by age groups (i.e., 18-29, 30-49, 50-59, and > 60 years). However, the findings for individuals $\leq 25$ years is not teased out from the data.

Lastly, five of the reviewed studies had adequate representation of Black participants: 1) all Blacks (Debnam et al., 2012), 2) 49.5\% Blacks (Hermstad et al., 2010), 3) 34.6\% Blacks (Rees et al., 2010), 4) 98\% Blacks (Thrasher et al., 2004), and 5) all Blacks (Watters \& Satia, 2009). Unfortunately, the mean age range for these five studies is 43.9 to 64.5 years. More research is needed regarding social support among individuals ages 18 to 25 years, specifically Black emerging adults. In Pender and colleagues' (2006) revised HPM, social support directly and indirectly (through 
commitment to a plan of action) influenced the behavior outcome. For this dissertation study, perceived family and friend social support may directly influence dietary fat intake in that as social support increases then the likelihood of individuals successfully managing their dietary fat intake also increases. This dissertation study's examination of social support and dietary fat intake among Black emerging adults can help to shed light on these gaps in the literature and the reported mixed findings. The findings from this dissertation study may help researchers develop social support interventions that help emerging adults manage their dietary fat intake.

\section{Perceived Generalized Stress and DFI}

Stress occurs when individuals perceive events, circumstances, and/or their environment to be taxing or overwhelming (Lazarus \&Folkman, 1984; Lyon, 2000; Vedhara \& Irwin, 2005). The cause of an individual's stress, known as a stressor, can be short term (acute stress) or occur daily (chronic stress) (Torres \& Nowson, 2007). Stressors can be positive (e.g., wedding) or negative (e.g., unemployment) (Cohen, 2000) and disturb the body's homeostasis (Selye, 1974; Vedhara \& Irwin, 2005). Exposure to stressors induces two responses: 1) a "fight-or-flight" response that occurs under acute stress situations/environments in which the sympathetic adrenal medullary system is activated that releases catecholamines (adrenaline and noradrenaline); and 2) a passive response that occurs under chronic, stressful situations/environments in which the hypothalamic-pituitary-adrenal (HPA) axis is hyperactive and releases corticosteroids (cortisol) (Rice, 2000; Torres \& Nowson, 2007). 
The body's response to acute and chronic stress may lead to physiologic changes, such as slowed gastric emptying and increase of blood pressure and heart rate (Torres \& Nowson, 2007). The body's stress response is beneficial in that it protects individuals from harmful situations and helps the body learn how to adapt and adjust its future response in stressful situations (Cohen, 2000; McEwen, 1998; Vedhara \& Irwin, 2005). However, repeated exposure to the same stressor may negatively affect the stress response by causing a dysregulation of the stress responsive system, which diminishes individuals' ability to continually adapt to and/or recover from the stressor and can lead to overproduction of glucocorticoids (Björntorp, 2001; Cohen, 2000; Dallman, 2010; Miller \& O'Callaghan, 2002; Vedhara \& Irwin, 2005).

There is conflicting data in the literature regarding whether or not stress influences individuals' quality of diet (e.g., fat intake) (Hargreaves et al., 2002; Wansink, 2006). Tucker, Weymiller, Cutshall, Rhudy, and Lohse (2012) found that healthpromoting behaviors had a significant negative relationship with stress; for example, the correlation between nutrition and stress was $-.31, p<.001$. Oliver and Wardle (1999) found that young adults perceive that their eating patterns are influenced by stress, although the direction of the stress effect varies; about equal numbers reported eating more (42\%) and eating less (38\%) when stressed. However, Dallman (2010) suggests that the excessive production of glucocorticoids increases caloric intake and one's desire for "comfort foods" and reinforces feeding habits (Björntorp, 2001; Dallman, 2010).

Stress has been associated with emotional and binge eating (Oliver et al., 2000; Sims et al., 2008; Wansink, 2006). In addition, persons feeling stressed tend to consume 
more than their recommended percentage of energy from fat (Cartwright et al., 2003; Contrada \& Baum, 2011; Dallman, 2010; Fowles et al., 2012; McCann, Warnick, \& Knopp, 1990; Ng \& Jeffery, 2003; Wardle, Steptoe, Oliver, \& Lipsey, 2000), overeat (Jeong \& Kim, 2007; Torres \& Nowson, 2007; Walcott-McQuigg, 1995), and make unhealthy food choices (i.e., fast food, which tends to be high in fat content) (Contrada \& Baum, 2011; Edmonds, 2010; Kandiah, Yake, Jones, \& Meyer, 2006; Oliver \& Wardle,1999; Steptoe, Lipsey, \& Wardle, 1998).

For example, Edmonds (2010) found in a sample of Black women, ages 22 to 86 years, a significant negative relationship between stress and health-promoting behaviors $(r=-.37, p<.05)$. Wardle et al. (2000), McCann et al. (1990), and Ng and Jeffery (2003) found that individuals consume more fat when stressed. Wardle et al. (2000) found that fat intake was higher under high-work stress periods ( $M=91.5$ grams/day) compared to low-work stress periods $(M=87.2$ grams/day) $(F[1,76]=3.58, p=.06)$ (Wardle et al., 2000). There was an increase in the amount of saturated fat intake ( $M=34.3$ grams/day) during high-work stress periods compared to low-work stress periods $(M=31.69$ grams/day) $(F[1,76]=7.54, p<.01)$ (Wardle et al., 2000). McCann et al. (1990) found similar results in that perceived stress was greater during a high-workload period compared to a low-workload period $(p<.05)$. During a high-workload period participants consumed more calories $(p<.05)$, greater amounts of total fat $(p<.05)$ and saturated fat $(p<.05)$, and greater percentage of calories from fat $(p<.05)$ as compared to a lowworkload period (McCann et al., 1990). Ng and Jeffery (2003) also found that greater perceived stress was positively associated with high fat intake $(\beta=.21, p<.01)$. 
Similar findings regarding stress and fat intake have been reported among younger populations. Cartwright and colleagues' (2003) examined the relationships between stress and dietary practices among a sample of schoolchildren in South London $(N=4,320)$ with $18.9 \%$ of the children being Black. They found that greater stress was positively associated with high fat food intake. Children who were the most stressed had greater odds of consuming more high fat foods $(\mathrm{OR}=1.93[1.60-2.34], p=.00)$ compared to children who were the least stressed. Although not a significant finding, in Fowles and colleagues' (2012) sample of pregnant women ages 16 and older (mean age $=24.7$ years, with $14 \%$ of the women being Black), high stressed participants consumed more than their recommended percentage of energy from fat (34\% of energy from fat) compared to normal stressed participants (32\% of energy from fat). Fowles et al. (2012) found a negative relationship between stress and diet quality $(r=-.35)$. These studies provide support that under stressful situations younger populations may consume foods high in fat.

However, there are gaps in the literature in regards to the relationship between stress and dietary fat intake. Ethnicity was not reported in some of the studies, which makes it difficult to determine if Blacks were underrepresented in their samples. In general, males in these studies are underrepresented. Lastly, most of the studies focus on unhealthy eating behaviors and stress (e.g., stress and overeating). Only five of the studies focused on stress and dietary fat intake (Cartwright et al., 2003; Fowles et al., 2012; McCann et al., 1990; Ng \& Jeffery, 2003; Wardle et al., 2000). Of these five studies, only two addressed stress and fat intake among younger populations (Cartwright 
et al., 2003; Fowles et al., 2012). The reviewed literature supports that stress may influence Black emerging adults' consumption of foods (high dietary fat intake), which may lead to long-term disease risks (i.e., cardiovascular disease). Using the HPM as a theoretical framework, perceived generalized stress may directly influence dietary fat intake in that as stress increases then the likelihood of individuals successfully managing their dietary fat intake decreases. This dissertation study's examination of perceived generalized stress among Black emerging adults addresses these gaps in the literature. The findings may help researchers develop culturally sensitive interventions to help this age group manage their stress and dietary fat intake.

\section{MODIFYING AND MEDIATING FACTORS OF DFI}

This current study examined the potential moderating effects of religious commitment and social support. Researchers have examined the moderating effects of religious variables. As already described, due to the practice of using religious terms interchangeably, studies pertaining to religion, religiosity, spirituality, and religious commitment are included. In general, researchers have found the above-mentioned religious variables to be significant protective factors (Allen et al., 2013; Bowen-Reid \& Harrell, 2002; Ellison, \& Henderson, 2011; Hill, Burdette, \& Idler, 2011; Joshi et al., 2009; Kilbourne, Cummings, \& Levine, 2011; Larson \& Larson, 2003; McDougle et al., 2013; Pargament, 1997). Individuals with greater levels of religion, religiosity, spirituality, and/or religious commitment reported better mental and physical health outcomes compared to individuals with less levels. However, Chung (2010) reported 
mixed findings in which some spirituality variables buffered the effects of stress and other spirituality variables did not. Ellison, Boardman, Williams, and Jackson (2001) found that religious involvement did not moderate the effects of stress on well-being in a sample of adults, ages 18 years and older $(n=1,139)$. Using the HPM as a theoretical framework, in this current study it is predicted that religious commitment may moderate perceived stress' effect on dietary fat intake.

Researchers have also examined the moderating effects of social support and found it to protect individuals from the adverse effects of their environments (e.g., stress) and risky health behaviors (Bloom, 1990; Connell \& D’Augelli, 1990; Ferguson \& Xie, 2012; Holt \& Espelage, 2007; Hughes et al., 2004; Wilks, 2008). A few researchers have examined the moderating effect of social support in food security, eating behaviors, and healthy eating studies, in which there are mixed findings. In the food security studies there were mixed findings regarding whether social support was a significant moderator (Muzoora, Martin, \& Weiser, 2012; De Marco \& Thorburn, 2009). Social support was a significant moderator in the studies regarding eating behaviors and healthy eating (McKinley, 2009; Wickrama et al., 2012).

However, little research has been done in examining the moderating effects of social support on dietary fat intake. In a study conducted by Burke, Beilin, Butt, Mansour, and Mori (2008) no significant interaction of social support was found for fat intake among a sample of adults ages 40 to 70 years. Using the HPM as a theoretical framework, in this current study it is predicted that social support may moderate individual characteristics' effect on dietary fat intake. 
As described in the above literature, little research has been conducted in examining the moderating effects of social support and religious commitment in regards to dietary fat intake. No studies were found for religious commitment. As for the moderating effects of social support, with dietary fat intake as the dependent variable, only one study, with a sample of adults ages 40-70, has been conducted by Burke et al. (2008). This is the first study, to date, to examine the potential moderating effects of religious commitment and social support in regards to dietary fat intake among Black emerging adults.

In regards to mediation, researchers have examined if stress explained the relationship between an independent variable and outcome. Little research has been done in examining stress' mediating effects in the area of dietary behaviors. However, Greenfield and Marks (2009) determined that stress mediated the relationship between physical and psychological violence experienced as a child and risk for obesity as an adult. Mulder, de Bruin, Schreurs, van Ameijden, and van Woerkum (2001) found that stress partially mediated the relationship between education and health behaviors, such as breakfast frequency and consumption of vegetables. This current study is the first study, to date, to examine the potential mediating effect of stress on the relationship between religious commitment and dietary fat intake. Musick and Worthen (2010) proposed a mediation framework model in which religion leads to social and psychological outcomes, which lead to better health outcomes. In this study, it is predicted that religious commitment leads to decreased perceived stress, which leads to decreased dietary fat intake. 


\section{SUMMARY}

Previous literature on Black emerging adults and their dietary fat intake, cultural food influences, selected predictor variables identified in the HPM (gender, BMI, income adequacy, religious commitment, perceived barriers for healthy eating, perceived selfefficacy related to managing DFI, perceived family social support, perceived friend social support, and perceived generalized stress) was reviewed. According to the literature, Blacks have high fat-related dietary behaviors, high fat intake, and a preference for high fat foods. These health behaviors could be in part due to their traditional diet and may increase their risk for CVD.

According to the reviewed literature, in general, males consumed more dietary fat compared to females, perceived barriers and stress had a positive relationship with dietary fat intake, and perceived self-efficacy had a negative relationship with dietary fat intake. There were mixed findings regarding the relationships between dietary fat intake and BMI, income adequacy, religious commitment, and social support. Researchers have found religious commitment and social support to protect individuals from the adverse effects of their environments (e.g., stress), meaning these variables were significant moderators. To this date, studies examining the potential moderating effects of religious commitment and social support and the mediating effects of stress in regards to dietary fat intake have not been conducted.

Most of the reviewed studies had older adult samples. In general, samples' mean age was greater than 40 years. When emerging adult populations were included in studies, the findings for individuals ages 18 to 25 years were not teased out from the 
reported data. Ethnicity was not reported in some of the studies, which makes it difficult to determine if Blacks were underrepresented in their samples. Furthermore, religious factors influencing dietary fat intake were difficult to assess, due to religious terms being used interchangeably. Guided by the HPM framework, the age- and ethnicity/race-gap in the literature is addressed by this current dissertation study. No studies, with a sample exclusively comprised of emerging adults, have examined the proposed factors' relationships with dietary fat intake. 


\section{Chapter 3: Methods}

For this chapter, the research design, sample, sample size, and power analysis are described. A detailed description of the measurement tools, methods of data collection, and data analyses are discussed. In addition, this chapter explains the procedures and results of a pilot study used for this dissertation. Lastly, ethical concerns and the protection of human subjects are examined.

\section{RESEARCH DESIGN}

This current study is categorized as non-experimental since the influences of the selected independent variables on dietary fat intake (DFI) were examined, the independent variables were not manipulated, and no controls were imposed (Keith, 2006; Kuzma \& Bohnenblust, 2005). The study is a cross-sectional, descriptive correlational research design. Since primary data was collected at one time period to determine the influences of the independent variables on participants' dietary fat intake, the study is considered cross-sectional (Polit \& Beck, 2012). Further, this study is descriptive correlational, designed to describe the relationships among independent variables and dependent variables (Polit \& Beck, 2012). A limitation to the research design selected for this current study is that causal inferences cannot be made (Polit \& Beck, 2012). However, the advantages of this particular research design are that it allows for a large amount of data to be collected, and it is an inexpensive, simple way to explore relationships (Polit \& Beck, 2012; Gravetter \& Wallnau, 2004; Kuzma \& Bohnenblust, 2005). 
Using a convenience sample, participants completed a confidential online survey consisting of a demographic questionnaire and eight instruments. Twenty-one percent of American Psychological Association journals have published at least one article that reported on Internet research, with $39 \%$ being correlational studies and $25 \%$ having a college student sample (Skitka \& Sargis, 2006). Some advantages to using the Internet for research include the time saved in collecting data, avoidance of waste and costs associated with paper instruments, easy to download data for analysis, and access to underrepresented samples (Fan \& Yan, 2010; Huntington et al., 2009; Skitka \& Sargis, 2006). Even though the Internet facilitates access to underrepresented samples, there is conflicting evidence for whether or not it gives researchers a diverse sample. For example, there is potential for less diversity in the sample since not everyone may have access to the Internet (Huntington et al., 2009), and the results of the study may not be generalized to the general population (Fan \& Yan, 2010). However, in 2009 the Pew Research Center (2015) reported that $93 \%$ of young adults (ages 18-29 years) go online (use the Internet). Daily use of the Internet ranged from 59-70\% among adults, ages 18 years and older: Whites (70\%), Blacks (61\%), and Hispanics (59\%) (Pew Research Center, 2015). Black young adults use the mobile web more than other ethnicities/races (Lenhart, Purcell, Smith, \& Zickuhr, 2010). In addition, Lewis, Watson, and White's (2009) study revealed that participants who completed an online survey were more demographically diverse than those who completed the paper and pencil version. These recent statistics support the use of online surveys with emerging adult populations. 
Other potential limitations include technical constraints (e.g., participant's ability to load the web link to access the survey), technical failure, computer virus, criminal activity via the Internet, type of Internet connection (e.g., dial-up connection tends to be slower compared to cable), and potential for invalid participant responses due to participants' inability to ask questions. There is also the possibility that someone besides the participant will complete the survey (e.g., participant has a friend take the survey for him/her). The web design can also be a limitation in that participants may end the survey early by misinterpreting the progress bar indicating completion of a section versus the survey. Modes of delivering the survey and reminding participants to complete the survey (e.g., spam-blocking features on the computer) may prevent the participant from receiving the web link for the survey (Fan \& Yan, 2010; Huntington et al., 2009; Skitka \& Sargis, 2006).

Despite these limitations, there is evidence of high response rates for online surveys among young adults (Larson et al., 2011). Generation Y, individuals ages 18 to 32 years (born between 1977-1990), has been nicknamed the "Net Generation" (Jones \& Fox, 2009). The Internet is used mostly by younger populations, with more than half of the adult users ranging from the age of 18 to 44 years (Jones \& Fox, 2009). According to the Pew Internet and American Life Project surveys from 2004 to 2009, ninety-three percent of individuals, ages 18 to 29, used the Internet (Pew Research Center, 2013b).

The survey program software used to design the survey for this current study is called Qualtrics (Qualtrics, 2014; University of Texas at Austin, 2014b). Qualtrics is able to support different browsers and formats (i.e., Excel and Statistical Package for the 
Social Science [SPSS] Windows), which may help to increase participants' access to the survey and allow for the data to be transferred for analysis (Fan \& Yan, 2010). The survey program software also has a "save and continue later" feature, which allowed participants to complete the survey in multiple sessions and increase the possibility of them answering all of the survey items. To decrease the risk of the data being compromised by an Internet virus or criminal activity, the data were stored on a secure server (Fan \& Yan, 2010). The collected data had one possible identifying item, the participants' email addresses. Therefore, once data collection was completed, with the Qualtrics online software, the data was transferred to a password-protected file for analysis.

\section{SAMPLE}

The population of interest for this present study was emerging Black adults. The convenience sample consisted of Black male and female participants, ages 18 to 25 years, who self-identify as Black and speak and read English. Participants were excluded if they were pregnant since their nutritional requirements are different due to their pregnancy. Participants living outside of the U.S. were also excluded.

Multiple recruitment strategies were used to obtain a sample frame that corresponded to the population. First, the investigator contacted some leaders of local Black churches. After explaining the purpose of the study and how email addresses would be protected, the investigator requested permission to obtain a list of email addresses for Black emerging adults within their congregation. Due to privacy concerns, 
some of the leaders refused to share such information. Those leaders who did not refuse, shared that a consolidated list of email addresses did not exist; but the leaders were willing to send the survey link out themselves to their contacts. Second, a few colleges/universities were contacted to obtain a list of email addresses for their Black students, ages 18 to 25 years. Due to colleges/universities being unable to release information based on race/ethnicity, a list of email addresses for Black students was not obtained. Some of the colleges/universities said they would send out the survey link to all of their students once the investigator received approval from their IRB. One of the contacted colleges/universities shared that a list of all of the students' email address could be obtained for a fee. The investigator was not able to draw from a random sample since there was not a sample frame available to use. Therefore, due to feasibility issues, the sampling procedure described in the next section was used.

\section{SAMPLE SIZE DETERMINATION}

The results from a pilot study conducted in 2013 by the investigator, which included all of the independent and dependent variables for this current study, were used to calculate the sample size in this study (Horton, 2013b). Religious commitment is one of the main independent variables of interests for this current study, and it is an understudied factor in the literature in regards to dietary fat intake. Thus, the correlation value for religious commitment and dietary fat intake from the pilot study $(r=.28)$ was used to calculate the estimated sample size. Based on the pilot study, an estimated sample size was calculated using G*Power (version 3.1.2) (Erdfelder, Faul, \& Buchner, 1996). 
The resulting sample size of 189 was determined to be sufficient to detect statistical significance. The following inputs were entered into $\mathrm{G}^{*}$ Power: linear multiple regression, fixed model, $R^{2}$ increase, significance level of $p=.05$, effect size of .09 , and power of .80 for 9 predictor variables. A significance level of $p=.05$ and power of .80 was used since they are the "conventional standard" for most research studies (Polit \& Beck, 2012). To account for potential missing data, additional participants were recruited for a sample size of 266 participants. Some participants were excluded, which is discussed further in Chapter 4. Thus, the final sample size for this dissertion study is 251.

\section{STUDY PROCEDURE}

\section{Sampling Procedure}

The investigator recruited participants primarily by advertisements distributed through sites frequented by Black emerging adults (i.e., flyers emailed through universities/colleges). The investigator recruited from February 27 to September 24, 2014. Recruitment occurred in the following ways: 1) face-to-face visits with organizations; 2) face-to-face visits with local salons and barbershops; 3) advertisement through social media sites; 4) advertisement through online classifieds; 5) email attachments sent to organizations; 6) word of mouth; and 7) snowball sampling.

The investigator's pre-established community partnerships through volunteer service and public health promotion events facilitated connections with organizations/businesses for recruiting potential participants (Yancey, Ortega, \& Kumanyika, 2006). Face-to-face visits entailed the investigator going to 
organizations/businesses in person to discuss the study and recruit potential participants. The investigator contacted the following organizations face-to-face:

- Local churches and associations (i.e., Grace Covenant Christian Center of Austin, Mt. Zion Baptist Church, St. James Missionary Baptist Church, and St. John Regular Baptist Association)

- Regional and national church associations (i.e., Missionary Baptist General Convention of Texas in Tyler, Texas and National Baptist Convention of America, Inc. in Memphis, Tennessee)

- Local and regional events (i.e., Juneteenth Celebrations in Round Rock, Texas; Kick and Roll 3-on-3 basketball fundraiser in Round Rock, Texas; and Relay for Life in Weatherford, Texas)

- Student organizations at The University of Texas at Austin (i.e., African Students Association, African American Nursing Students Association, Black Health Professions Organization, Black Student Alliance, National Association of Black Journalists, National Society of Black Engineers, and Umoja)

- Local salons and barbershops (e.g., Ann's Private Cuts, Gaston “This Is It” Barber and Beauty, and Brown's Barber Shop).

Researchers have shown that the above-mentioned strategies are effective in recruiting Black emerging adults (Henry \& Eastell, 2000; UyBico, Pavel, \& Gross, 2007; Wallace, McLellan-Lemal, Harris, Townsend, \& Miller, 2011; Yancey et al., 2006).

When contacting these organizations/businesses, the investigator sent via email 75 
information about the purpose of the study, web link to the survey, procedures, and incentive for completing the survey. The leader, manager, or owner of the organization/business was followed up with by an additional email to see if they had any questions regarding the study. If the leader, manager, or owner of the organization/business permitted the investigator to come to one of its meetings (e.g., student organization meeting) to explain the study face-to-face to its members/patrons, then a convenient time was arranged for such a meeting.

Upon receiving permission from the organization to meet with its members and the business owner to come to his/her establishment, the investigator explained the study and the importance of problem (dietary fat intake and CVD), which included reviewing the information in the cover letter and answering any questions. Permission was requested from the organization/business to recruit potential participants, post a copy of the flyer (see Appendix E), and distribute postcards (see Appendix F). The organization/business were also asked to distribute the flyer through their email distribution list. The flyer included information regarding the web link for the survey, purpose of survey, estimated time to complete the survey, and contact information of the investigator. Just in case recipients did not open the flyer attachment when it was distributed through the organization/business's email distribution list, the web link, embedded within the text of the emailed message, was provided to organizations (see Appendix G). Individuals who wished to participate in the study accessed the web link provided in the flyer, postcard, cover letter, and emailed message. If the organization/business gave permission and time was available, the investigator had an 
electronic tablet (i.e., iPad) or laptop computer with Internet connection available during face-to-face visits just in case potential participants decided to complete the survey at the time of the face-to-face visit.

When the investigator was unsuccessful in coordinating face-to-face visits with organizations/businesses, information about the study was sent via email to the leader or manager of the organization/business. In the email, the organization/business was asked to distribute the information and attached flyer through their email distribution list. The flyer included information regarding the web link for the survey, purpose of survey, estimated time to complete the survey, and contact information of the investigator. The web link was embedded within the text of the emailed message (see Appendix G).

Face-to-face visits with organizations/businesses helped to facilitate building trust between the investigator and the members of the organization/business. It also allowed the investigator to clarify questions potential participants had about the study. These faceto-face visits made use of an important feature-people connected in a network of relationships (Salganik \& Heckathorn, 2004). These networks of relationships lead to others being invited to participate in the study by their peers, which Salganik and Heckathorn (2004) call respondent-driven sampling.

Advertisement through social media sites and online classifieds (i.e., Facebook and Craigslist) is appropriate for this study in that over half of the adult Internet population is between the ages of 18 and 44 years (Jones \& Fox, 2009), and social media sites are popular for this age group (Subrahmanyam, Reich, Waechter, \& Espinoza, 2008). Along with using the Internet for entertainment, this age group uses the Internet to 
keep track of and communicate with friends and family, read and post on other people's blogs, use social networking sites, create social profiles, send instant messages, search for information, buy products, and handle banking transactions (Jones \& Fox, 2009; Qualman, 2013). There is evidence to support social media sites as an option in assessing health behaviors among young populations (Ramo \& Prochaska, 2012). In addition, Feman et al. (2008) recommend using Internet advertising to recruit participants, particularly posting classifieds on free websites. When Feman et al. (2008) evaluated the effectiveness of recruitment methods they found that Internet advertising had the highest enrollment rate compared to flyers/brochures, mass transit (e.g., subway lines), and physician referrals.

The investigator advertised through the social media site called Facebook (Facebook, 2013). Facebook is one of the most common social media sites being used today (Close, 2012). There is an estimated 600 million users of Facebook, which is up from 150 million in 2009 (Close, 2012). A profile on Facebook was created for the study, which had 220 friends and 46 likes during its 7 months of activation and recruiting period. The web link to the survey and a copy of the flyer and cover letter was posted on Facebook. Individuals who wished to participate in the study accessed the web link embedded in the Facebook page.

As for advertising on classified sites, Craigslist was used, which is a free classifieds site and has been ranked in the top 100 websites in the world (Traffic Pillar, 2008). Each month over 20 million individual visit the Craigslist website (Traffic Pillar, 2008). This site has been recommended for targeted online advertisements within the 
U.S. (Business.com, 2013; McCoy, 2015). Appropriate subsections under the "community" category were selected to post advertisements for the study (McCoy, 2015; Traffic Pillar, 2008). Information from the cover letter was included in the advertisement. The investigator used Soulciti.com (Griotmedia, 2014) and the University of Texas Events Calendar (University of Texas at Austin, 2014a), which are also free classified sites. Individuals who wished to participate in the study accessed the web link embedded in the advertisements.

Using methods such as social media and online classifieds sites lead to snowball sampling recruitment, which is also known as network sampling or chain sampling (Polit \& Beck, 2012). Snowball sampling recruitment is when a participant provides a list of friends for the investigator to contact as potential participants and then these potential participants provide a list of their friends to contact and this process continues to repeat (Goodman, 1961; Polit \& Beck, 2012; Sadler, Lee, Lim, \& Fullerton, 2010). Due to the trustworthiness of the original participant, snowball sampling recruitment increased the likelihood that identified friends may participate in the study (Sadler et al., 2010).

In addition, the investigator completed and submitted IRB applications for Austin Community College, Huston-Tillotson University, Spelman College, and Xavier University. Spelman College was the only IRB application that was not approved in time for data collection. Documentation for IRB approval for the other above-mentioned institutions is in Appendix O. All of the above applications were approved. HustonTillotson University and Xavier University sent the survey link via email twice to all of their enrolled students. Austin Community College did not send the survey link via email, 
but gave the investigator permission to recruit on their campuses. The survey link was not sent via email by Spelman College to its students since the IRB approval from came shortly after data collection was completed.

A pilot study conducted in 2013 by the investigator (Horton, 2013b), tested the above advertising methods for recruitment this current study. In the pilot study participants were asked, "How did you hear about this survey?" As described in Table 1, most of the participants, who completed the survey, heard about the pilot study through "word of mouth" and email. Similarly, participants were asked the same question in this current dissertation study. Most of the participants in this current study heard about the study through a recruitment site (i.e., university/college and faith-based organization), followed by email and word of mouth (Table 1). A detailed description of participants obtained from each recruitment site (i.e., university) is described in Table 14 (Chapter 4). 
Table 1. Pilot Study and Dissertation Study Advertisement

\begin{tabular}{lllllll}
\hline \multicolumn{7}{c}{ Type of Advertisement } \\
\hline Study & Recruitment Site & Email & Word of mouth & Craigslist & Facebook \% & Text message \\
$\%(\mathrm{n})$ & $\%(\mathrm{n})$ & $\%(\mathrm{n})$ & $0(\mathrm{n})$ & $8(2)$ \\
\hline Pilot & N/A & $36(9)$ & $44(11)$ & $0(0)$ & $12(3)$ & $8(2)$ \\
Dissertation & $33.70(122)$ & $29.28(106)$ & $18.23(66)$ & $11.33(41)$ & $3.31(12)$ & $1.10(4)$ \\
\hline
\end{tabular}

Note. Participants were allowed to select more than one option for how they heard about the study. Recruitment site data was not collected during the pilot study. 


\section{Data Collection Procedures}

Once the University of Texas at Austin's Institutional Review Board (IRB) approved the study, then participant recruitment commenced. The study was advertised as previously described. Interested recruits accessed the web link provided to them.

The participants accessed the web link by typing or copying and pasting the web link into their Internet browser. The web link was obtained from the flyer, postcard, email, Facebook, Craigslist, Soulciti.com, the University of Texas at Austin Events Calendar, and/or word of mouth. The investigator had an electronic tablet (i.e., iPad) or laptop computer with Internet connection available during face-to-face visits with various organizations/businesses just in case potential participants decide to complete the survey at the time of the face-to-face visit. When participants accessed the web link, a consent form to participate in Internet research was on the opening page of the survey (see Appendix B). If they agreed to participate in the study, they clicked the button, located at the bottom of the consent form, next to the statement "Yes, I read and understand the consent. I agree to participate in this study." An option button was available for participants to click in order to print a copy of the consent for their own record, which included contact information for the investigator and IRB. If participants did not want to participate in the study, they clicked the button, located at the bottom of the consent form, next to the statement "I do not agree to participate in this study." When they clicked this button it exited them out of the survey browser. If participants gave consent to participate in the study, the next page assessed whether or not participants met the inclusion criteria: live in the U.S., read and speak English, self-identify as Black, age 18 to 25 years, and 
not pregnant. The online survey program was not able to capture participants' birthday and year for the inclusion criteria question-design. Therefore, if participants were born between 1989-1996 at the time of the survey, they met the age criteria. If participants met all of the inclusion criteria, then they were allowed to proceed with the rest of the survey. However, if they did not meet the criteria, they received a message thanking them for their interest, explaining that they did not meet the research study requirements, and encouraging them to share the web link with those who meet the inclusion criteria.

An online survey company, Qualtrics, was used to collect data (Qualtrics, 2014; University of Texas at Austin, 2014b). The survey consisted of a demographic questionnaire and eight instruments (see Appendix C). There was a progression or advancement bar to indicate participants' progress through the survey. When they reached the end of the survey, a page opened explaining they have completed the survey. If participants wanted to receive the results of their dietary fat intake screening, there was an option on the last page of the online survey for them to click "Yes, send me results". If they clicked yes, then they were sent their results via email, along with web links to education information on healthy eating (see Appendix H). In addition, if participants felt distressed by the questions in the survey, there was an option on the last page of the online survey for them to click "Yes, send me mental health resources". If they clicked yes, participants were sent these resources via email (see Appendix I).

Based on the findings of a pilot study conducted in 2013 by the investigator (Horton, 2013b), the online survey for this current study was estimated to take 15-25 minutes for participants to complete. Therefore, the online survey was designed to give 
participants the opportunity to stop and complete it at a later time. This save and continue later feature allowed participants to complete the survey in multiple sessions, if needed. The online survey was designed to track participants' completion of the survey. The investigator would periodically log into the survey site to assess participants' completion of the survey. If the participants had started the survey but had not completed it, they were sent short personalized emails, including the web link to the survey, reminding them to complete the survey (see Appendix J) (Edwards et al., 2009; Fan \& Yan, 2010). If needed, a total of two reminders were sent to participants needing to complete their survey.

As an incentive for completing the survey (Edwards et al., 2009; Fan \& Yan, 2010), participants received a $\$ 1.29$ value Amazon.com gift card to use towards a music download (Amazon.com, 2013). Each participant was sent an electronic Amazon.com gift card for $\$ 1.29$, with a message thanking him or her for participating in the study. The gift card was sent through Amazon.com to the email address provided by the participant in his/her survey. The gift cards were purchased with funds from the Good Samaritan Scholarship that the investigator was awarded.

A paper version of the survey was given to participants during face-to-face visits upon request or when there was poor Internet connection at a particular site. The paper version of the survey had all of the same items as the online version, except it did not ask for participants' email address since the participants returned the survey to the investigator in person. Prior to participants completing a paper survey the investigator gave them the first page of the survey to read, which was the consent form (see Appendix 
K). After they read the consent form and the investigator answered any questions they had, participants were asked if they still want to participate in the study. If they said "no," then the investigator thanked them for their interest and time in reading the consent form. If the participants said "yes" to participating in the study, then they were given the paper version of the online survey to complete.

The participants were encouraged to keep the consent form for their own record. Same as the online survey, participants completing the paper survey were be able to choose to receive their dietary fat intake screening results and/or a list of mental health resources. If participants wanted to receive the results of their dietary fat intake screening and/or a list of mental health resources, there was an option for them to check the box labeled "Yes". When participants turned in their completed survey in person to the investigator, the investigator assessed how they answered the questions regarding whether or not they wanted to receive their dietary fat intake screening results and/or a list of mental health resources before participants left the face-to-face site. If they answered "yes" to the dietary fat intake screening results, then the investigator went to the 17 questions in the survey that assessed their dietary fat intake (i.e., Block Dietary Fat Screener, which will be described later in this chapter), calculated their dietary fat intake, and gave them their results and printed education information on healthy eating (see 


\section{Appendix L).}

If they answered "yes" to the list of mental health resources, then participants were given a printed list of mental health resources (see Appendix I) before participants left the face-to-face site. Lastly, upon turning in their completed survey to the investigator, participants signed their name next to the $\$ 1.29$ value Amazon.com gift card serial number, which they received as an incentive for completing the survey (see Appendix N). This signed list of received gift cards serves as documentation that participants received a gift card. This gift card list and paper surveys are stored in a locked filing cabinet accessible only by the investigator.

The online survey company, Qualtrics, maintained the participants' online responses to the survey for the period of the study on a secure server. Once all the participants completed the survey the investigator downloaded the de-identified data, with removed email addresses, into a password-protected Excel file. The participants, who completed the paper version of the online survey, were assigned a numerical code and their survey data was manually entered into a password-protected Excel file. Then the two Excel files (online and paper surveys) were combined into one Excel file. This combined Excel file was then transferred as a file in Statistical Package for the Social Science (SPSS) Windows (version 21) for data analysis (IBM Corporation, 2011). The SPSS file contains participants' numerical code and their responses to the survey.

The SPSS file was saved onto a password-protected computer owned by the investigator. It was also saved to a separate password-protected flash drive in case the investigator's computer crashed. The flash drive was kept locked in a file cabinet drawer 
located in the investigator's office. After transfer of the data into a SPSS file, the data was deleted from the online server. Data analysis was conducted on a passwordprotected computer. The web link for the survey was set up to close, including a closure explanation message for visitors to the web link, once 272 participants had completed it.

\section{INSTRUMENTATION}

The online survey consisted of a demographic questionnaire and eight established instruments to measure variables in the HPM: 1) the Block Dietary Fat Screener (BDFS) (Block, Gillespie, Rosenbaum, \& Jenson, 2000); 2) the Economic Adequacy Scale (EAS) (Marie Lobo, personal communication, February 11, 2013); 3) Intrinsic Religiosity Subscale from the Duke University Religion Index (DUREL) (Koenig \& Büssing, 2010); 4) the Healthy Foods and Snacks-Barriers Subscale from the Motivators of and Barriers to Health-Smart Behaviors Inventory (MB-HSBI) (Tucker et al., 2011); 5) the Resisting Relapse Subscale from the Self-efficacy for Eating Behaviors Scale (SEEBS) (Sallis, Pinski, Grossman, Patterson, \& Nader, 1988); 6) the Family Support for Heart Healthy Eating Habits Scale (Family SHHEHS) (Sallis, Grossman, Pinski, Patterson, \& Nader, 1987); 7) the Friend Support for Heart Healthy Eating Habits Scale (Friend SHHEHS) (Sallis et al., 1987); and 8) the Perceived Stress Scale (PSS) (Cohen, Kamarck, \& Mermelstein, 1983). The demographic questionnaire and eight established instruments were in English. Each item/question on the demographic questionnaire and eight instruments were keyed into the online survey. The online survey was estimated to take 
15-25 minutes to complete. Table 2 is a list instruments used in this current study and the variables from the HPM measured.

Table 2. Instruments and Variables

\begin{tabular}{ll}
\hline Instrument & Measured Variable \\
\hline Block Dietary Fat Screener & Dietary fat intake \\
Economic Adequacy Scale & Income adequacy \\
Intrinsic Religiosity Subscale & Religious commitment \\
$\begin{array}{l}\text { Healthy Foods and Snacks- } \\
\text { Barriers Subscale }\end{array}$ & Perceived barriers for healthy eating \\
Resisting Relapse Subscale & $\begin{array}{l}\text { Perceived self-efficacy related to managing } \\
\text { dietary fat intake }\end{array}$ \\
$\begin{array}{l}\text { Family Support for Heart Healthy } \\
\text { Eating Habits Scale }\end{array}$ & Perceived family support \\
$\begin{array}{l}\text { Friend Support for Heart Healthy } \\
\text { Eating Habits Scale }\end{array}$ & Perceived friend support \\
Perceived Stress Scale & Perceived generalized stress \\
\hline
\end{tabular}

\section{Block Dietary Fat Screener (BDFS)}

\section{Description}

Dietary fat intake was measured by the total score on the Block Dietary Fat Screener (BDFS) (see Appendix C). The BDFS is a 17-item self-report measure to assess the percentage of total fat calories consumed by an individual. Participants answered items about how often they ate certain foods within a particular time-period. Their responses were based on a 5-point Likert-type scale: 0 (1 time a month or less), 1 (2-3 
times a month), 2 (1-2 times a week), 3 (3-4 times a week), and 4 (5 or more times a week). Final scores, calculated by summing the points for all 17 items, can range from 0 to 68. The total is called the fat score. Higher scores indicate a greater percentage of total calories come from fat intake. Lower scores indicate less percentage of total calories come from fat intake (Block et al., 2000).

The fat score can be categorized into four levels of percentage of total fat calories consumed: scores ranging from 0 to 7 are considered to be very low fat intake (probably less than $25 \%$ of calories); scores ranging from 8 to 14 are considered to be average fat intake (probably 30-35\% of calories); scores ranging from 15 to 22 are considered to be quite high fat intake (probably $>35 \%$ calories); and scores $\geq 23$ are considered to be very high fat intake (probably 40-50\% calories) (Block et al., 2000). These categories were used in describing the characteristics of the study's sample.

Food frequency questionnaires do not report reliability measures. According to Fitzpatrick (1998), reliability is the "consistency of responses on self-report, normreferenced measures of attitudes and behavior" (p. 483). Self-report responses of food behaviors may vary (i.e., seasonal foods and eating patterns on the weekends). Thus, since measures of food behaviors would not be consistent each time a participant completes a food frequency questionnaire, reliability is not used to judge the quality of food intake measures. Instead, "it is important and desirable that any new dietary assessment method be validated or calibrated against other more established methods" (Coulston, Rock, \& Monsen, 2001, p. 18), which the authors of the BDFS did, as described below. 


\section{Validity}

The 1995 Block 100-item Food Frequency Questionnaire, a "gold standard," was used to assess concurrent criterion-related validity for the BDFS. Employees of a company in California completed both the 1995 Block 100-item Food Frequency Questionnaire and the BDSF. The participants were from diverse ethnic backgrounds, ranging in age from 20 to 69 years $(N=208)$ (Block et al., 2000).

Spearman rank-order correlation coefficients were used to assess the validity between the BDFS and the 1995 Block 100-item Food Frequency Questionnaire. The BDFS was good at ranking participants for several nutrients. For fat elements the Spearman rank-order correlation coefficients were $\geq .60(p=.0001)$ for total fat (grams per day), saturated fat (grams per day), monounsaturated fat (grams per day), dietary cholesterol (micrograms per day), and percent fat (daily percent of total calories). Particularly, BDFS significantly ranked participants' percentage of total fat calories $(N=$ 208, rho $=.63, p=.0001)$ similarly to the 1995 Block 100-item Food Frequency

Questionnaire. The resulting correlations suggest the BDFS can provide a reasonable estimate of participants' percentage of fat intake. In addition, the predictive scores on the BDFS were good at determining the correct category of percent of energy from fat compared to the 1995 Block 100-item Food Frequency Questionnaire (i.e., very high fat intake) (Block et al., 2000).

Concurrent criterion-related validity for the BDFS has also been done using an average of three 24-hour dietary recalls (Toobert et al., 2011). Along with the 24-hour dietary recalls, a total of 205 overweight or obese adults, living in Oregon, completed 
computerized and paper versions of fruit, vegetable, and fat screening instruments (Toobert et al., 2011). The BDFS had a significant correlation with the multiple 24-hour recalls regarding fat intake $(r=.50, p=<.05)$ (Toobert et al., 2011). Based on the above findings and its development among a diverse ethnic sample that had emerging adults, the BDFS is considered a valid instrument to measure dietary fat intake for this current study.

\section{Demographic Questionnaire}

The demographic questionnaire was used to describe the sample. It measured age, gender, education, marital status, living arrangement, employment, and self-reported height and weight, which were used to calculate BMI (see Appendix C). Among the above listed variables, gender and BMI are individual characteristics in the theoretical framework.

\section{Body Mass Index (BMI)}

Body mass index, classified as an individual characteristic in the theoretical framework, was measured in this study. It was used to estimate the amount of body fatness in individuals (CDC, 2015b). Positive relationships have been found between BMI, CVD, and dietary behaviors (Canfi et al., 2011; Juonala et al., 2011; Ledikwe et al., 2003; Maskarinec et al., 2000; Liu et al., 2012; Miller et al., 2012; Newby et al., 2003; Taylor et al., 2010; Vernarelli et al., 2011). Participants reported their height in feet and inches (to the nearest $1 / 2$ inch) and weight in pounds (to the nearest pound) on the demographic questionnaire. Body mass index was then be calculated from the participants' self-report height and weight by converting their weight to kilograms and 
height to meters. These converted values were used in the following formula: BMI = (weight in kilograms/height in meters ${ }^{2}$ ) (CDC, 2015b).

There are some limitations to using self-reported height and weight for research studies. Generally, individuals tend to overestimate their height and underestimate their weight (Brener, McManus, Galuska, Lowry, \& Wechsler, 2003; Bowman \& DeLucia, 1992; Bowring et al., 2012; Flood, Webb, Lazarus, \& Pang, 2000; Gorber, Tremblay, Moher, \& Gorber, 2007; Huber, 2007; Krul, Daanen, \& Choi, 2010; Lim, Seubsman, \& Sleigh, 2009; Stommel \& Schoenborn, 2009; Stunkard \& Albaum, 1981; Taylor et al., 2006; Vriendt, Huybrechts, Ottevaere, Van Trimpont, \& De Henauw, 2009). Mixed results exist on which populations overestimate height the most. However, Stommel and Schoenborn (2009) found that overestimation of height was greater among Blacks compared to other races/ethnicities, and Flood et al. (2009) found an overestimation of height among older populations compared to younger populations. Weight tends to be underestimated among both men and women, college educated individuals, and overweight and obese individuals (Bowman \& DeLucia, 1992; Flood et al., 2009; Gorber et al., 2007; Lim et al., 2009; Taylor et al., 2006). With the possibility of individuals providing inaccurate self-reported height and weight, then the resulting BMI calculations may be underestimated (Craig \& Adams, 2009; Flood et al., 2009; Gorber et al., 2007; Krul et al., 2010; Taylor et al., 2006).

Although there are some limitations to using self-reported height and weight, selfreported height and weight are highly correlated with measured values and remain accurate tools for assessing population-based estimates of obesity (Bowman \& DeLucia, 
1992; Lim et al., 2009; Stommel \& Schoenborn, 2009; Stunkard \& Albaum, 1981; Taylor et al., 2006). Researchers have shown supportive evidence for the validity of selfreported height and weight in the classification of overweight and obese individuals (Brener et al., 2003; Bowring et al., 2012; Huber, 2007). Individuals who weigh themselves at least once a month may be more likely to report accurate weights compared to those who weigh less frequently (Flood et al., 2009). Therefore, questions were embedded into the demographic questionnaire to assess the last time participants weighed themselves, frequency of weighing themselves, and how they perceive their weight (e.g., underweight, normal weight, and overweight) (Musick, 2004). These additional questions may help to improve the accuracy of the self-reported data (see Table 14, Chapter 4). Individuals who weigh themselves at least once a month may be more likely to report accurate weights compared to those who weigh themselves less frequently (Flood et al., 2000).

Despite its limitations, BMI was selected to use because it is an easy method to assess for overweight and obesity among populations (CDC, 2015b). In addition, the use of self-reported height and weight is fairly accurate in classifying individuals in BMI categories (e.g., overweight and obese). One only needs to have the participants' selfreported height and weight to calculate BMI, which makes BMI easy to use. Since the study design collected data via the Internet, measured height and weights would not be feasible. 


\section{Economic Adequacy Scale \\ Description}

Since income can be a determinant in whether or not individuals consume healthy diets (Darmon \& Drewnowski, 2008; Inglis et al., 2009; Raffensperger et al., 2010), income adequacy, an individual characteristic in the theoretical framework, was measured in this study. The total score on the Economic Adequacy Scale (EAS) measured income adequacy (see Appendix C). The EAS is a self-report measure to assess if individuals have adequate income to meet their daily living (Marie Lobo, personal communication, February 11, 2013). The EAS consists of two general questions related to having adequate income to meet one's daily needs and then five questions addressing the following areas: rent, food, health, recreation, and child care. The responses are based on a 4-point Likert-type scale: 4 (more than adequate), 3 (adequately), 2 (less than adequate), and 1 (not at all). Final scores, calculated by summing the points from the seven questions, can range from 7 to 28 , with higher scores indicating greater perceptions of having adequate income to meet one's daily needs. Lower scores indicate perceptions of having less than adequate income to meet one's daily needs.

\section{Validity}

According to the instrument's author, a review of family and parenthood literature was used to develop the EAS. After its development, a panel of nurse researchers reviewed it, and based on the expert panels' judgment, no alterations were made to the instrument after its initial development (Marie Lobo, personal communication, February 11, 2013). Thus, content validity was established. 


\section{Reliability}

The EAS was originally tested in two White samples: families who considered themselves healthy and first-time pregnant couples (Marie Lobo, personal communication, February 11, 2013). When the EAS was developed in 1982 its internal consistency was $.92(n=132$, White first-time pregnant couples) and .94 ( $n=163$, White families who considered themselves healthy) (Marie Lobo, personal communication, February 11, 2013). The EAS has been tested in populations with disabilities as described by Becker and Stuifbergen (2004): multiple sclerosis $(n=577)$, postpolio syndrome $(n=$ $1,730)$, and polio survivors without postpolio syndrome $(n=423)$. In all three samples the Cronbach's alpha was 0.96 (Becker \& Stuifbergen, 2004).

Although the EAS has been mostly tested in populations that were primarily White and/or had disabilities, in 1987 McCain tested the EAS in a sample of participants who had a premature infant $(N=82)$, consisting of $25 \%$ Black, and the Cronbach's alpha was .87 (as cited by Marie Lobo, personal communication, February 11, 2013). In a pilot study conducted by the investigator (Horton, 2013b), the Cronbach's alpha was .89 ( $n=$ 24, Black emerging adults). The Cronbach's alpha for this current study was $.91(N=$ 251).

The EAS has been tested in populations living in rural and urban geographical locations, such as rural residents in a cardiac rehabilitation program $(N=254)$ (Johnson, Weinert, \& Richardson, 1998). It has also been tested among participants with multiple sclerosis $(N=807)$ in which a majority of the participants lived in metropolitan areas (Stuifbergen, 1999). In addition, the EAS has been used in a study that included younger 
participants such as Killien, Habermann, and Jarrett's (2001) sample of postpartum mothers $(N=149)$ ranging from age 20 to 41 years.

Since emerging adults may be living independently on their own (e.g., no financial support from their parents/guardians) or partially to totally dependent upon their parents/guardians' income, emerging adults may not know their family income. The EAS has been selected for this study because it bypasses the need for emerging adults to obtain income data from their parents/guardians, which participants may not want to share and/or parents/guardians may not share with them. In addition, the investigator is interested in whether or not emerging adults perceive their income to be adequate enough to eat healthy.

\section{Intrinsic Religiosity (IR) Subscale of the Duke University Religion Index \\ Description}

Religious commitment reflects the influence of religious beliefs and teachings upon individuals' decisions and behaviors (Koenig et al., 2001). Religious commitment, also sometimes referred to as intrinsic religiosity, may influence one's eating behaviors (Chester et al., 2006). Perhaps individuals' internal commitment to their religious beliefs motivates them to practice healthy behaviors (e.g., manage dietary fat intake). Religious commitment, classified as an individual characteristic in the theoretical framework, was measured in this study. Religious commitment was measured by the score on one of the subscales (described below) from the Duke University Religion Index (DUREL) (see Appendix C). The DUREL was originally designed for Western religions, such as Christianity and Judaism (Koenig \& Büssing, 2010). It has been used in over 100 
published studies and translated into 10 languages (Koenig \& Büssing, 2010). Therefore, the IR subscale from the DUREL was used because of its use with diverse samples, which included emerging adults.

The DUREL is a self-report measure that assesses three dimensions of religiosity: organizational religious activity, non-organizational religious activity, and intrinsic religiosity (Koenig \& Büssing, 2010). Organizational religious activity describes an individual's participation in religious group activities (i.e., prayer group and group bible study) or religious activities occurring in public settings (i.e., attending religious services-church) (Koenig \& Büssing, 2010). Non-organizational religious activity describes an individual's participation in private religious activities (i.e., prayer and watching a religious television program) (Koenig \& Büssing, 2010). Intrinsic religiosity (IR) describes an individual's level of religious commitment or motivation (Koenig \& Büssing, 2010).

These three dimensions are also the three subscales on the DUREL. The DUREL is a 5-item instrument: organizational religious activity subscale (one item), nonorganizational religious activity subscale (one item), and IR subscale (three items) (Koenig \& Büssing, 2010). This current study used only the IR subscale. The IR subscale reflects the influence of religious beliefs and teachings upon individuals' behaviors (Koenig et al., 2001). It is possible that religious commitment may also influence eating behaviors. If individuals' religious beliefs include caring for their bodies as "temples," then they may be more likely to eat healthy foods. Healthy eating behaviors (e.g., lower fat intake) have been found among some religious groups (e.g., Seventh-Day Adventists) 
(Koenig et al., 2001). Using a 5-point Likert scale, 1 (definitely not true of me) to 5 (definitely true of me), participants rate their level of agreement with each item. The score for the IR subscale is calculated by summing all the points from the three items, which can range from 3 to 15 . Higher scores on the IR subscale indicate more religious commitment and lower scores indicate less religious commitment.

\section{Validity}

During 1997-1998 when Koenig was developing the DUREL, he selected three items from the Hoge Intrinsic Religiosity Scale to make the IR subscale, based on their "loading on the intrinsic factor, correlation with the total score, and relationship with health outcomes" (as cited in Koenig \& Büssing, 2010, p. 80). Concurrent validity was established between 1997-1998 by Koenig in the original Duke Hospital Study with inpatients ages 60 and older, in which the Hoge Intrinsic Religiosity Scale strongly correlated $(r=.85)$ with the IR subscale (Koenig \& Büssing, 2010).

Storch et al. (2004a) conducted a study with two samples: 635 undergraduate students (72\% White, $12 \%$ Black, $11 \%$ Hispanic, and 4\% Asian; $M$ age $==20.60$ years, $S D=2.80)$ and 244 undergraduate students (67\% White, 14\% Black, 10\% Hispanic, and 5\% Asian, $M$ age $==20.76$ years, $S D=1.55$ ). Using factor analysis with varimax rotation, Storch et al. (2004a) reported construct validity of the DUREL. They found that all five items on the DUREL had the presence of one factor, which accounted for $76 \%$ of the variance and had an eigenvalue of 3.81 (Storch et al. (2004a). Factor loadings of the DUREL ranged from .853 to .907 . Koenig and Büssing (2010) reported that other researchers have found high convergent validity of the DUREL with other measures of 
religiosity ( $r=.71$ to .86 ). The IR subscale was selected for this current study because its three items logically fit the theoretical framework and focuses on individuals' religious beliefs and experiences (i.e., my religious beliefs are what really lie behind my whole approach to life).

\section{Reliability}

The DUREL has acceptable internal consistency values. Koenig and Büssing (2010) reported the DUREL's Cronbach's alphas ranged from .78 to .91, which in general are considered acceptable coefficient values (Fitzpatrick, 1998). The Cronbach's alpha for the IR subscale was .75 (Koenig \& Büssing, 2010). In a pilot study conducted by the investigator (Horton, 2013b), the Cronbach's alpha for the IR subscale was .71 ( $n$ $=33$, Black emerging adults). The Cronbach's alpha for this current study was $.90(N=$ 251).

As part of a larger study, Storch, Strawser, and Storch (2004b), established the test-retest reliability of the DUREL among 20 undergraduate students $(M$ age $=24.7$ years, $S D=5$ ) attending a public university in the southeastern U.S. The ethnicity/race categories of the samples were the following: 79\% White, 11\% Asian, 5\% Black, and 5\% Hispanic. The retests occurred within two weeks after the initial test. The resulting oneway random effects intraclass correlation coefficient obtained for the DUREL was .91 (Storch et al., 2004b). 


\section{Healthy Foods and Snacks-Barriers Subscale on the Motivators of and Barriers to Health-Smart Behaviors Inventory \\ Description}

Barriers may prevent individuals from eating healthy (Pender, 1996). Therefore, perceived barriers for healthy eating, a behavioral-specific cognitions and affect factor in the theoretical framework, was measured in this study. Perceived barriers for healthy eating was measured by the score on the Healthy Foods and Snacks barriers subscale on the Motivators of and Barriers to Health-Smart Behaviors Inventory (described below) (see Appendix C).

The Motivators of and Barriers to Health-Smart Behaviors Inventory (MB-HSBI) is a self-report measure to assess the motivators of and barriers to "health-smart behaviors", which are positive dietary (e.g., eating foods low in fat) and physical activity (e.g., engaging in moderate-intensity physical activity for at least 150 minutes per week) health-promoting behaviors (Tucker et al., 2011). The MB-HSBI consists of four domains: Healthy Breakfast, Healthy Drinks, Healthy Foods and Snacks, and Physical Activity. Each of these domains is divided into two subscales: motivators and barriers, for a total of eight subscales. This current study used one of the subscales called the Healthy Foods and Snacks to assess barriers only. The barrier subscale for this domain consists of 15 items. Using a 4-point Likert scale, 1 (strongly disagree) to 4 (strongly agree), participants rate their level of agreement with each item as a personal barrier to engaging with the identified "health-smart behavior". The score for the Healthy Foods and Snacks barriers (HFS Barriers) subscale is calculated by summing all the points from 
the 15 items, scores can range from 15 to 60 . Higher scores on the MS-HSBI indicate more barriers to engaging in "health-smart behaviors" related to eating; lower scores indicate fewer barriers. The HFS Barriers subscale consists of three subscales: negative attitudes (eight items), availability (three items), and self-control (four items) (Tucker et al., 2011). The score for the entire HFS Barriers subscale was used for this study, which is the sum of the items on the negative attitudes, availability, and self-control subscales.

The HFS Barriers subscale from the MB-HSBI was used for this study because of its cultural diversity and identified barrier items. The MB-HSBI was developed with individuals from diverse cultural backgrounds and included emerging adults. Its barrier items are similar to the barriers identified in the pilot study for the proposed study and the literature review related to fat intake for this study (e.g., taste preferences, availability, and access). In addition, the HFS Barriers subscale has acceptable internal consistency values.

\section{Validity}

The MB-HSBI was developed in a sample of adults from diverse cultural backgrounds and low-income households (Tucker et al., 2011). Researchers conducted six focus groups in which participants, ages 18 to 89 years, reported their own motivators of and barriers to "health-smart behaviors". Their responses formed the items on the preliminary MB-HSBI (Tucker et al., 2011). Then the researchers had lay community members and health professionals from culturally diverse backgrounds review the items for content validity (Tucker et al., 2011). Next the researchers pilot-tested the MB-HSBI with 70 adults, ages 20 to 78 years, of which 16 were Black (Tucker et al., 2011). Based 
on the pilot-test, Tucker et al. (2011) made revisions and then tested the revised MBHSBI in a larger sample $(N=926)$, which included Black participants $(36 \%)$.

Using factor analysis, the highest loading items were included in the revised version of the MB-HSBI, which resulted in the HFS Barrier subscale having three interpretable factors: negative attitudes, availability, and self-control (Tucker et al., 2011). The HFS Barrier subscale had loadings ranging from .52 to .82 (Tucker et al., 2011). The loadings' percentage of variance accounted for was not reported.

Convergent and discriminant validity was established for the HFS subscale by examining the correlations between the motivator subscale and the barrier subscale. As expected, both subscales were negatively correlated with each other ( $r=-.37, n=369$ ) (Tucker et al., 2011). As expected, the interpretable factors within the barrier subscale (negative attitudes, availability, and self-control) had negative correlations with the factors within the motivator subscale (routine, availability, health benefits, medical issues, and convenience) ( $r=-.01$ to $-.46, n=369)$ (Tucker et al., 2011). In addition, there were higher correlations found between motivator subscales and barrier subscales within a specific health-smart domain compared to the set of cross-correlations. For example, all of the correlations for the HFS Motivator subscale and HFS Barrier subscale were higher than the set of cross-correlations within the Healthy Foods and Snacks domain (Tucker et al., 2011).

The MB-HSBI's criterion-related validity was established by comparing it against two other measures: 1) the Physical Health subscale of the School Health Efficacy Questionnaire and 2) the Health-Smart Behavior Goal Agreement Ratings. The 
correlation between the Physical Health subscale of the School Health Efficacy Questionnaire and the HFS barrier subscale was -.37; a negative correlation would be expected (Tucker et al., 2011). Individuals with higher self-efficacy had fewer barriers. The correlations between the Health-Smart Behavior Goal Agreement Ratings items that related to positive dietary health-promoting behaviors (i.e., eating a healthy breakfast, fruit, vegetables, whole grains, and lower calorie foods/snacks and drinking water and other healthy drinks are personal goals) and the HFS Barrier subscale yielded small correlations ranging from -.06 to -.17 (Tucker et al., 2011). Individuals with higher goal ratings (i.e., eating a healthy breakfast is a personal goal) had fewer barriers. The authors did not report statistical significance, which makes the interpretation of those correlations inconclusive.

\section{Reliability}

The initial MB-HSBI was pilot-tested and refined using a national sample of culturally diverse adults, ages 20 to 78 years (Tucker et al., 2011). The internal consistency for the entire MB-HSBI ranged from .78 to .92 (Tucker et al., 2011). The Cronbach's alpha coefficient for the HFS Barrier subscale was .85 (Tucker et al., 2011). In a pilot study conducted by the investigator (Horton, 2013b), the Cronbach's alpha for the HFS Barrier subscale was .72 ( $n=26$, Black emerging adults). The Cronbach's alpha for this current study was $.82(N=251)$. Since the MB-HSBI is a newly developed instrument the above coefficient values are considered acceptable (Fitzpatrick, 1998). 


\section{Resisting Relapse Subscale of the Self-Efficacy for Eating Behaviors Scale \\ Description}

Self-efficacy can influence one's ability to manage dietary fat intake (AbuSabha \& Achterberg, 1997; Armitage \& Conner, 1999; Grembowski et al., 1993; Nothwehr, 2004; Povey et al., 2000; Watters \& Satia, 2009). Therefore, perceived self-efficacy related to managing dietary fat intake, a behavioral-specific cognitions and affect factor in the theoretical framework, was measured in this study. Perceived self-efficacy related to managing dietary fat intake was measured by the score on the resisting relapse subscale of the Self-Efficacy for Eating Behaviors Scale (SEEBS) (see Appendix C).

The SEEBS is a self-report measure to assess how confident participants think they could perform a behavior for at least six months related to changing their eating habits (Sallis et al., 1988). The SEEBS consist of five subscales that assess self-efficacy for a total of 89 items: 1) Resisting Relapse; 2) Reducing Calories; 3) Reducing Salt; 4) Reducing Fat; and 5) Behavioral Skills (Sallis et al., 1988). For this current study only one subscale, the resisting relapse subscale, was used because its items relate more to dietary fat intake compared to the reducing calories, reducing fat, and reducing salt subscales.

Both the resisting relapse and reducing fat subscales were used in a pilot study conducted by the investigator (Horton, 2013b). In order to lessen the burden of the survey, in regards to time, the investigator decided to use only the resisting relapse subscale in this current study. The items on the reducing fat subscale appear to assess self-efficacy for dietary behaviors such as cut down on gravies and cream sauces, eat 
poultry without skin, and bake food instead of frying. The items on the resisting relapse subscale appear to assess self-efficacy for managing dietary fat intake such as stick to low-fat foods when dining with friends, stick to low-fat foods when at a party, and avoid eating fast food for meals. In addition, the Cronbach's alpha for the resisting relapse subscale $(\alpha=.78, n=12)$ was higher than the reducing fat subscale $(\alpha=.65, n=18)$ in the pilot study (Horton, 2013b).

The resisting relapse subscale consists of 18 items. However, since the item "drink fewer diet drinks with sodium" does not pertain to low-fat dietary habits it was not included, resulting in only 17 items to be used from the resisting relapse subscale. For the resisting relapse subscale, when terminology is used specifically for a meal (e.g., lunch) then the term "meals" was substituted.

The resisting relapse subscale has a 5-point Likert scale, from 1 (sure I could not do it) to 5 (sure I could do it), including an option to select "does not apply" (Sallis et al., 1988). The score for the resisting relapse subscale is calculated by summing all the points from the 17 items, which can range from 17 to 85 (Sallis et al., 1988). Higher scores on the resisting relapse subscale indicate greater self-efficacy for managing dietary fat intake; lower scores indicate less self-efficacy.

The SEEBS was used for this study because of the appropriateness of the resisting relapse subscale in assessing perceived self-efficacy related to managing dietary fat intake and its use in diverse populations. The SEEBS has been used in samples that included Blacks and emerging adults. In addition, the resisting relapse subscale has acceptable test-retest reliability coefficient values and internal consistency. 


\section{Validity}

During the initial development of the SEEBS, Black, Hispanic, and White participants, age 45 years and younger, were interviewed to determine behavioral change items for the instrument $(N=40)$. Then content validity was established when a panel of researchers reviewed the items for final selection and wording. Construct validity was established by factor analysis with varimax rotation in which five factors accounted for $44 \%$ of the variance and had eigenvalues $>2$ (Sallis et al., 1988). The factor loadings for the 18 items in the resisting relapse subscale ranged from .46 to .73 and accounted for $26.3 \%$ of the variance (Sallis et al., 1988).

In addition, construct validity was also established by comparing the Multidimensional Health Locus of Control (MHLC) scale scores (internal, chance, and external subscales) with the SEEBS. The following correlations were found for resisting relapse $(N=171)$ : MHLC internal subscale $(r=.32, p=.001)$, MHLC chance subscale $(r$ $=-.20, p=.01)$, and MHLC external subscale ( $r=-.11$, NS) (Sallis et al., 1988).

Concurrent criterion-related validity was established by comparing self-efficacy factor scores from the SEEBS with participants' reported diet habits (i.e., food frequency questionnaire "not heart healthy/heart healthy" dietary index). Using the food frequency

questionnaire, participants rated their frequency of consuming select food items. A dietitian then categorized each food item as "heart healthy" (e.g., low fat) or "not heart healthy" (e.g., high fat) and created a heart healthy index, in which scores ranged from .08 to 10.50 (Sallis et al., 1988). Higher scores on the heart healthy index indicated a high-fat and high-sodium diet (Sallis et al., 1988). Sallis et al. (1988) evaluated whether 
or not participants' reported food frequency questionnaire outcomes correlated with the self-efficacy factors in the SEEBS, which were all statistically significant. The correlation for the resisting relapse subscale and heart healthy index was $r=-.37$ ( $p<$ .001) Sallis et al., 1988). Individuals with higher heart healthy index scores (e.g., high fat) had less self-efficacy in managing their dietary fat intake.

Using one-way analyses of variance, Sallis et al. (1988) compared participants' reported diet behavior changes to their self-efficacy factor scores across groups. There was significant mean differences between participants who were "trying to eat less fat" and those who were not "trying to eat less fat" for the self-efficacy factor of resisting relapse $(N=171, F=9.15, p<.01)$ (Sallis et al., 1988). This significant comparison supports the validity of the SEEBS.

\section{Reliability}

The initial study for the development of the SEEBS was with participants age 45 years old and younger $(N=40)$ and included participants who were Black, Hispanic, and White (Sallis et al., 1988). The SEEBS has also been used in other studies in which the samples included Blacks (Nabi \& Thomas, 2012; Timmerman \& Brown, 2012) and emerging adults (Nabi \& Thomas, 2012). Reliability was established using test-retest reliability. In a study of 171 participants, of which a majority were college students $(n=$ 154), the resulting one to two week test-retest reliability coefficients for the five subscales of the SEEBS ranged from .85 to .93 (Sallis et al., 1988).

The internal consistency, as measured by the Cronbach's alpha, for the resisting relapse subscale was .93 $(N=171)$ (Sallis et al., 1988). Grizzle (2009) used 20 items 
from the SEEBS in studying the eating habit confidence among 289 police officers, ages 22 to 65 years. The majority of Grizzle's (2009) sample was Black (58.7\%). The resulting Cronbach's alpha coefficient for this study was .94 (Grizzle, 2009). In addition, Nabi and Thomas (2012) conducted a study with 253 females ages 18 to 23 years, of which $4 \%$ were Black, to assess how food advertisements influenced self-efficacy. The resulting Cronbach's alpha coefficients for the entire SEEBS scale ranged .67 to .89 (Nabi \& Thomas, 2012). As reported earlier, in a pilot study conducted by the investigator (Horton, 2013b), the Cronbach's alpha for the resisting relapse subscale was .78. The Cronbach's alpha for this current study was .93 $(N=251)$.

\section{Family Support for Heart Healthy Eating Habits Scale}

\section{Description}

Emerging adults receive social support from multiple sources to help them perform health-promoting behaviors (Pender et al., 2006). They have eating behaviors that were shaped by their families; however, their close connection with their friends can also influence their eating behaviors. Therefore, the social support emerging adults receive from their family and friends was examined separately in this study. Perceived family and friend social support, both behavioral-specific cognitions and affect factors in the theoretical framework, were included in the current study because they may facilitate or hinder behaviors associated with managing dietary fat intake (Feunekes et al., 1998; Fowles et al., 2012; Hart et al., 2007; Silverman et al., 2002; Thrasher et al., 2004).

Perceived family social support was measured by the total score on the Family Support for Heart Healthy Eating Habits Scale (Family SHHEHS) (see Appendix C). The 
Family SHHEHS is a 13-item self-report measure to assess the frequency of social support related to diet habits received from family in the last three months. The Family SHHEHS consists of two factors: encouragement and sabotage. The responses are based on a 5-point Likert-type scale: 1 (none), 2 (rarely), 3 (a few times), 4 (often), and 5 (very often). Final scores, calculated by summing the points from the 13 items, can range from 13 to 65 . The sabotage items were reverse scored when summing all the items on the Family SHHEHS. Higher total scores indicate greater family support and lower scores indicating less family support (Sallis et al., 1987).

\section{Validity}

Forty participants of diverse ethnic backgrounds, who were in the process of making dietary and/or exercise changes and age 45 years and younger, were interviewed to determine how family and friends had been supportive and unsupportive of participants' dietary changes and how participants would like their family and friends to be supportive in the future. The responses from the interviews were used to develop the items on the Family SHHEHS. Once the draft Family SHHEHS was developed it was pilot-tested with 171 participants, which included 154 college students (Sallis et al., 1987).

A dietitian categorized the participants' 24-hour diet recall interview items into heart healthy (i.e., low in fat) and not heart healthy (i.e., high in fat) (Sallis et al., 1987). This heart healthy/not heart healthy dietary index was used to assess concurrent criterionrelated validity. The encouragement factor on the Family SHHEHS was significantly 
correlated with the heart healthy/not heart healthy dietary index $(r=-.27, N=171, p<$ .001) while the sabotage factor was not significantly correlated (Sallis et al., 1987).

Construct validity was established by factor analysis. The factor analysis revealed two factors in the Family SHHEHS: "encouragement" (six items) and "sabotage" (seven items), in which the factor loadings ranged from .43 to .79 (Sallis et al., 1987). Encouragement and sabotage accounted for $42 \%$ of the variance (Sallis et al., 1987).

Specificity of the social support factors was determined when the participants were categorized into specific dietary-behavior changes (i.e., "trying to eat less fat"). Family social support factor scores were compared across categories using one-way analyses of variance, which revealed the following for family social support for eating factors related to dietary-change variables $(N=171)$ : "trying to eat less fat" and encouragement $(F=16.3, p<.001)$, "trying to eat less fat" and sabotage $(F=3.64$, not significantly related), "trying to eat fewer calories" and encouragement $(F=7.81, p<$ $.001)$, and "trying to eat fewer calories" and sabotage $(F=5.94, p<.05)$ (Sallis et al., 1987). Therefore, because of the sample used during development, which included Blacks and emerging adults, and adequate internal consistency, the Family SHHEHS was used for this current study.

\section{Reliability}

Reliability was established using test-retest reliability, in which the retests occurred within one to two weeks. The resulting test-retest reliability coefficients obtained for the Family SHHEHS was .86 $(p<.001)$ for encouragement and .57 for sabotage $(p<.001)$ (Sallis et al., 1987). Sallis et al. (1987) reported internal consistency, 
measured by Cronbach's alpha, for all the instruments in their study ranged from .61 to .91. The internal consistency for the Family SHHEHS subscales were the following ( $N=$ 171): encouragement $(\alpha=.87)$ and sabotage $(\alpha=.83)$ (Sallis et al., 1987), which are acceptable coefficient values (Fitzpatrick, 1998). In a pilot study among Black emerging adults $(n=24)$ conducted by the investigator (Horton, 2013b), the Cronbach's alpha obtained for the Family SHHEHS was .73. The Cronbach's alpha for this current study was .69 $(N=251)$.

\section{Friend Support for Heart Healthy Eating Habits Scale \\ Description}

Perceived friend social support was measured by the total score on the Friend Support for Heart Healthy Eating Habits Scale (Friend SHHEHS) (see Appendix C). The Friend SHHEHS is a 10-item self-report measure to assess the frequency of social support related to diet habits received from friends in the last three months. The Friend SHHEHS consists of two factors: positive comments and negative comments. The responses are based on a 5-point Likert-type scale: 1 (none), 2 (rarely), 3 (a few times), 4 (often), and 5 (very often). Final scores, calculated by summing the points from the 10 items, can range from 10 to 50 . The negative comment items were reverse scored when summing all the items on the Friend SHHEHS. Higher total scores indicate greater support from friends and lower scores indicate less support from friends (Sallis et al., 1987). 


\section{Validity}

The same forty participants, interviewed in the above Family SHHEHS, were used for the development of the items on the Friend SHHEHS. Once the draft Friend SHHEHS was developed, it was pilot-tested with the same 171 participants in the above Family SHHEHS, which included 154 college students. The heart healthy/not heart healthy dietary index developed above in the Family SHHEHS was used to assess concurrent criterion-related validity for the Friend SHHEHS. The positive comments factor on the Friend SHHEHS was significantly correlated with the heart healthy/not heart healthy dietary index $(r=-.19, N=171, p<.01)$ and the negative comments factor was not significantly correlated (Sallis et al., 1987).

In contrast, the Social Support Questionnaire did not significantly correlate with participants' reported dietary habits (Sallis et al., 1987). However, construct validity was established by factor analysis. The factor analysis revealed two factors in the Friend SHHEHS: "positive comments" (six items) and "negative comments" (four items), in which the factor loadings ranged from .54 to .84 and accounted for $40 \%$ of the variance (Sallis et al., 1987).

Specificity of the social support factors was determined when the participants were categorized into specific dietary-behavior changes (i.e., "trying to eat less fat"). Friend social support factor scores were compared across categories using one-way analyses of variance, which revealed significant findings for friend social support for eating factors related to dietary-change variables $(N=171)$ : "trying to eat less fat" and positive comments $[F=23.75, p<.001]$, "trying to eat less fat" and negative comments 
$[F=5.84, p<.05]$, "trying to eat fewer calories" and positive comments $[F=18.57, p<$ $.001]$, and "trying to eat fewer calories" and negative comments $[F=5.79, p<.05])$ (Sallis et al., 1987).

\section{Reliability}

Reliability was established using test-retest reliability, in which the retests occurred within one to two weeks. The resulting test-retest reliability coefficients obtained for the Friend SHHEHS was .81 for positive comments $(p<.001)$ and .78 for negative comments $(p<.001)$. Sallis et al. (1987) reported internal consistency, measured by Cronbach's alpha, for all the instruments in their study ranged from .61 to .91 . The internal consistency for the Friend SHHEHS subscales were the following $(N=171)$ : positive comments $(\alpha=.87)$ and negative comments $(\alpha=.80)$ (Sallis et al., 1987), which are acceptable coefficient values (Fitzpatrick, 1998). In a pilot study among Black emerging adults $(n=24)$ conducted by the investigator (Horton, 2013b), the Cronbach's alpha obtained for the Friend SHHEHS was .66. The Cronbach's alpha for this current study was .63 $(N=251)$.

Both the Family SHHEHS and the Friend SHHEHS were developed with a sample that included Blacks. In addition, both scales have been used in other studies that had Blacks in the samples (Horton, 2013b; Kelsey et al., 1996; Kiernan et al., 2012; Wilson \& Ampey-Thornhill, 2001). These instruments have also been used in samples that included emerging adults (Horton, 2013b; Kelsey et al., 1996; Kiernan et al., 2012). 


\section{Perceived Stress Scale (PSS)}

\section{Description}

Stressful situations can influence one's ability to manage dietary fat intake (Fowles et al., 2012; Ng \& Jeffery, 2003). Therefore, perceived generalized stress, a behavioral-specific cognitions and affect factor in the theoretical framework, was measured in this study. Perceived generalized stress was measured by the total score on the Perceived Stress Scale (PSS) (see Appendix C).

The PSS is a 10-item self-report measure to assess how participants appraised situations as stressful during the last month. The responses are based on a 5-point Likerttype scale: 0 (never), 1 (almost never), 2 (sometimes), 3 (fairly often), and 4 (very often). Final scores, calculated by summing the points from the 10 items, can range from 0 to 40 . The positively stated items on the PSS were reverse scored. Higher total scores indicate greater perceived stress and lower scores indicate less perceived stress (Coehen et al., 1983).

\section{Validity}

In addition to the study conducted by Coehen et al. (1983), the PSS has been tested in other groups that included Black and/or emerging adult samples (Chang, Banks, \& Watkins, 2004; Sellers, Caldwell, Schmeelk-Cone, \& Zimmerman, 2003; Sellers \& Shelton, 2003; Strogatz et al., 1997). The PSS was designed to be used in samples with at least a junior high school education. During its development three samples, two consisting of college students and one heterogeneous smoking-cessation group, were used to collect validation data for the PSS. The two college samples completed five 
scales: measurement of life events, social anxiety, depressive symptoms, and physical symptoms and the PSS. The smoking-cessation group completed a measurement of lifeevents and physical symptoms and the PSS (Cohen et al., 1983).

Using these measurements, concurrent and predictive validity was established. The PSS was significantly correlated to the measurement of life events: college student sample one $(n=332, r=.20, p<.01)$, smoking cessation sample at the beginning of treatment $(n=64, r=.38, p<.01)$, and smoking cessation sample at the end of treatment $(n=64, r=.39, p<.01)$ (Cohen et al., 1983). The PSS was significantly correlated to the measurement of the impact of life events in both college student samples $(r=.24-.35, p<$ $.01)$ and both smoking cessation samples $(r=.33-.49, p<.01)$ (Cohen et al., 1983).

The PSS was significantly correlated with social anxiety in both college student samples $(r=.37-.48, p<.001)$ (Cohen et al., 1983). In addition, the PSS had significant correlations in predicting depressive symptoms, as expected in both college student samples $(r=.65-.76, p<.001)($ Cohen et al., 1983). It also had a significant correlation in predicting physical symptoms in both college student samples $(r=.52-.65, p<.001)$ and the smoking cessation sample at the beginning of treatment $(n=64, r=.70, p<.001)$ (Cohen et al., 1983).

\section{Reliability}

Internal consistency was established using Cronbach's alphas. The Cronbach's alpha coefficient for the PSS in the college student samples ranged from .84 to .85 (Cohen et al., 1983). The Cronbach's alpha coefficient in the smoking-cessation sample was .86 (Cohen et al., 1983). According to Fitzpatrick (1998), these coefficient values are 
acceptable. In a pilot study among Black emerging adults $(n=23)$ conducted by the investigator (Horton, 2013b), a Cronbach's alpha of .87 was obtained. The Cronbach's alpha for this current study was $.87(N=251)$.

Due to its wide use in the literature and internal consistency, the PSS was used for this study. The PSS was developed with samples that included emerging adults. It has also been used in samples that included Blacks.

\section{PILOT STUDY}

A pilot study was conducted by the investigator from August through September 2013 among Black emerging adults, ages 18 to 25 living in the U.S. A total of 195 potential participants viewed the online survey. Sixty-three attempted to complete the online survey but only 42 were maintained for the pilot because the other 21 participants did not meet the inclusion criteria: live in the U.S., self-identify as Black, age 18 to 25 years, and not pregnant. Of the 42 participants, 24 participants completed the entire online survey and 18 participants partially completed the online survey. The data for all 42 participants was used for data analysis. The purpose of the pilot study was to refine and evaluate select instruments (acceptability, perceived usefulness, clarity) and examine factors that may influence dietary fat intake.

\section{Sample in the Pilot Study}

As mentioned above, 42 participants completed some or all of the online survey. The mean age was $22(S D=2.36)$ and of those who reported their gender $(n=31)$, there were 6 male and 27 female participants. A majority of the sample was single $(94.1 \%)$, 
highly educated in that they had completed some college or graduated from college. According to the BMI categories of the CDC (2015b), of the 34 participants who reported their height and weight to calculate BMI, a majority of the sample was overweight and/or obese (53\%): normal 44\% (BMI 18.5 to 24.9), overweight 18\% (BMI 25 to 29.9), and obese $35 \%(\mathrm{BMI} \geq 30)$. This finding matched the participants' perception of their weight, which $59 \%$ perceived themselves to be overweight and/or very overweight. Refer to Table 3 for additional demographic characteristics of the sample. 
Table 3. Demographic Characteristics of Pilot Study Sample

\begin{tabular}{lll}
\hline $\begin{array}{l}\text { Demographic Characteristic } \\
(n=34)\end{array}$ & Categories & $\%(n)$ \\
\hline Education Level & High School & $11.8(4)$ \\
& Some College & $35.3(12)$ \\
& Associate Degree & $5.9(2)$ \\
& Bachelor's Degree & $38.2(13)$ \\
& Graduate Degree & $8.8(3)$ \\
Marital Status & Single & $94.1(2)$ \\
& Married & $5.9(32)$ \\
Has Children & Yes & $2.9(1)$ \\
& No & $97.1(33)$ \\
Living Arrangement & Lives Alone & $20.6(7)$ \\
& Lives with spouse/partner, & $76.5(26)$ \\
& family, or roommate & \\
& Other & $2.9(1)$ \\
Employment Status & Full-time & $38.2(13)$ \\
& Part-time & $32.4(11)$ \\
& Self-employed & $2.9(1)$ \\
& Unemployed & $17.6(6)$ \\
Participants' Perception of Weight & Other & $8.8(3)$ \\
& Very Underweight & 0 \\
& Somewhat Underweight & $11.7(4)$ \\
& Just About Right & $29.4(10)$ \\
& Somewhat Overweight & $38.2(13)$ \\
& Very Overweight & $20.6(7)$ \\
\hline
\end{tabular}

Note. Not all percentages add up to $100 \%$ due to rounding.

\section{Procedure in the Pilot Study}

The pilot study was approved by the University of Texas at Austin's IRB. Participants were recruited primarily by advertisements distributed through sites frequented by Black emerging adults. Recruitment occurred in the following ways: advertisement through local churches (e.g., flyer posted at Mt. Zion Baptist Church), 
social media site (Facebook), and online classifieds (e.g., Craigslist), email of flyers, and word of mouth. The flyer included information regarding the web link for the survey, purpose of survey, estimated time to complete the survey, and contact information for the investigator.

Individuals who wished to participate in the study accessed the web link embedded in the above listed advertisements. They accessed the web link by typing or copying and pasting the web link into their Internet browser. When they accessed the web link, a consent form to participate in Internet research was on the opening page of the online survey. If they agreed to participate in the study, they clicked the button, located at the bottom of the consent form, next to the statement "Yes, I read and understand the consent. I agree to participate in this study." There was a statement at the end of the consent encouraging participants to print a copy of the consent for their own record. The consent included contact information for the investigator and IRB. After participants gave consent, the next pages of the online survey assessed whether or not participants meet the inclusion criteria: live in the U.S., read and speak English, self-identify as Black, age 18 to 25 years, and not pregnant. If participants met all of the inclusion criteria, then they will be allowed to proceed with the rest of the survey. However, if they did not meet the criteria, they received a message thanking them for their interest, explaining that they did not meet the research study requirements, and encouraging them to share the web link with those who met the inclusion criteria.

If they met the inclusion criteria, they proceeded through the survey. The survey consisted of a demographic questionnaire and nine instruments. The demographic 
questionnaire and nine instruments were same as the ones described earlier in this chapter, with the exception of the following additional instruments: Spiritual Well-being Scale (Ellison, 1983), Reducing Fat subscale from the SEEBS (Sallis et al., 1988), and General Ethnic Discrimination Scale (Landrine, Klonoff, Corral, Fernandez, \& Roesch, 2006). There was a progression or advancement bar to indicate participants' progress through the survey. When they reached the end of the survey a page opened explaining they had completed the survey, and their feedback was needed to improve the wording and clarity of the survey. The participants then answered the following questions to assess what they thought about the survey:

- How did you hear about this survey? (e.g., word of mouth, flyer, Facebook)

- How much time did it take you to complete this survey?

- What did you think about the length of the survey?

- How can the instructions on the survey be improved?

- Were there any questions you did not understand? If so, which ones?

- Were there any questions that made you feel uncomfortable and/or did not like? If so, which ones?

- Did you feel distressed by any of questions? If so, which ones?

- What other suggestions do you have to improve the wording and clarity of this online survey?

After these questions, there was a section on the last page of the survey with options for the participants to select whether or not to receive their dietary fat intake screening results and/or mental health resources. Those who requested their dietary fat 120 
intake screening results and/or mental health resources entered their email address and the investigator sent them the requested information. As an incentive for completing the survey, participants were given an option to be entered into drawings for a chance to win one of three $\$ 10$ value gift cards to Amazon.com.

In order not to link the participants to their data, their names and email addresses were maintained separate from the survey data in a separate site on the online server. The participants were assigned a numerical code. Thus, when the data from the online server were downloaded there was not any identifying information for the participants. The data were kept on a password protected computer and flash drive owned by the investigator. The flash drive was stored in a locked filing cabinet accessible only by the investigator.

Using SPSS Windows, version 20 (IBM Corporation, 2011), the data were analyzed with the level of statistical significance for all of the research questions set at $p$ $<.05$. Descriptive statistics were used to describe characteristics of the pilot study's sample (i.e., frequencies, percents, means, and standard deviations). Correlation coefficients were used to examine the relationships between the independent variables and dependent variable. Cronbach's alpha coefficients for reliability were determined for the following instruments:

- Block Dietary Fat Screener (Block et al., 2000)

- Economic Adequacy Scale (Marie Lobo, personal communication, February $11,2013)$

- Intrinsic Religiosity Subscale (Koenig \& Büssing, 2010)

- Spiritual Well-being Scale (Ellison, 1983) 
- Healthy Foods and Snacks Barriers subscale (Tucker et al., 2011)

- Resisting Relapse subscale (Sallis et al., 1988)

- Reducing Fat subscale (Sallis et al., 1988)

- Family Support for Heart Healthy Eating Habits Scale (Sallis et al., 1987)

- Friend Support for Heart Healthy Eating Habits Scale (Sallis et al., 1987)

- Perceived Stress Scale (Cohen et al., 1983)

- Appraisal of Stressful Situation Subscale from the General Ethnic Discrimination Scale (Landrine et al., 2006).

Content analysis was used to analyze participants' reported barriers to managing fat intake and their feedback (Green \& Thorogood, 2009).

\section{Findings of the Pilot Study}

The correlation coefficients for independent and dependent (dietary fat intake) variables ranged from -.42 to .28 . There were not any statistically significant correlations between the independent and the dependent variables. Although not statistically significant, the largest correlation between the independent variables and DFI was -.402 (family social support), which suggests that participants with less family social support tended to consume more dietary fat. As expected, the direction of the correlations for perceived barriers and perceived stress was in the positive direction, and friend social support was in the negative direction. These results indicate that participants with more barriers and stress tended to consume more dietary fat. Participants with less friend social support also tended to consume more dietary fat. Surprisingly, the correlations for selfefficacy and stress related to racism were not in an expected direction. Participants with 
more self-efficacy tended to have higher dietary fat intake; and participants with less stress related to racism tended to have lower dietary fat intake.

In order to examine the reliability of the above mentioned instruments, the Cronbach's alpha coefficients were used to assess internal consistency. As seen in Table 5 the reliability estimates ranged from .65 to .91 . With the exception of two, these coefficients suggest an acceptable internal consistency in the instruments used in the pilot study (Fitzpatrick, 1998). The reducing fat subscale $(\alpha=.65)$ and Friend SHHEHS $(\alpha=$ .66) may be reasonable for a sample size this small. The item analysis, as depicted in Table 6, shows some improvement in reliability estimates for item deletions; particularly two items were identified for each scale. Based on these results, the investigator will consider deleting items 7 and 8 on the Friend SHHEHS for this current dissertation study. 
Table 4. Correlation Matrix for Pilot Study Variables

\begin{tabular}{|c|c|c|c|c|c|c|c|c|c|c|c|c|c|c|}
\hline Variable & 1 & 2 & 3 & 4 & 5 & 6 & 7 & 8 & 9 & 10 & 11 & 12 & 13 & 14 \\
\hline 1. Gender & 1.00 & & & & & & & & & & & & & \\
\hline 2. BMI & .074 & 1.00 & & & & & & & & & & & & \\
\hline $\begin{array}{l}\text { 3. Religious } \\
\text { Commitment }\end{array}$ & -.086 & -.109 & 1.00 & & & & & & & & & & & \\
\hline 4. DFI & $-.015^{\S}$ & -.148 & .280 & 1.00 & & & & & & & & & & \\
\hline 5. Income Adequacy & .069 & .090 & .316 & .256 & 1.00 & & & & & & & & & \\
\hline 6. Spirituality & .147 & -.210 & .265 & .086 & .330 & 1.00 & & & & & & & & \\
\hline 7. Perceived Barriers & -.084 & .255 & $.375^{*}$ & .231 & -.161 & -.163 & 1.00 & & & & & & & \\
\hline $\begin{array}{l}\text { 8. Self- Efficacy } \\
\text { Resisting Relapse }\end{array}$ & .135 & .073 & .295 & .161 & .171 & .143 & .247 & 1.00 & & & & & & \\
\hline $\begin{array}{l}\text { 9. Self- Efficacy } \\
\text { Reducing Fat }\end{array}$ & .073 & $.468^{*}$ & -.042 & .005 & .001 & .099 & -.150 & .020 & 1.00 & & & & & \\
\hline $\begin{array}{l}\text { 10. Self-Efficacy Total } \\
\text { Score }\end{array}$ & .152 & .291 & .234 & .141 & .149 & .172 & .140 & $.873^{* * *}$ & $.506^{*}$ & 1.00 & & & & \\
\hline $\begin{array}{l}\text { 11. Family Social } \\
\text { Support }\end{array}$ & .249 & -.217 & -.210 & -.402 & -.003 & .001 & -.339 & .161 & .040 & .158 & 1.00 & & & \\
\hline $\begin{array}{l}\text { 12. Friend Social } \\
\text { Support }\end{array}$ & -.210 & .029 & -.054 & -.188 & -.085 & -.111 & .017 & -.026 & -.014 & -.029 & .283 & 1.00 & & \\
\hline 13. Perceived Stress & .167 & .154 & .002 & .104 & -.403 & -.294 & .262 & .103 & .157 & .167 & .022 & -.126 & 1.00 & \\
\hline $\begin{array}{l}\text { 14. Perceived Stress } \\
\text { Related to Racism }\end{array}$ & .078 & .379 & -.097 & -.100 & .289 & -.344 & -.017 & -.104 & .218 & .017 & .102 & .000 & .252 & 1.00 \\
\hline
\end{tabular}

Note. The above coefficients are Pearson's r. A Pearson's r was conducted for gender according to the IBM (n.d.) instructions for obtaining a point biserial correlation $(\S) . * p<.05 \quad * * p<.01 \quad * * * p<.001$ 
Table 5. Cronbach's Alpha Coefficients for the Instruments and Subscales

\begin{tabular}{lll}
\hline Instrument & Number of items & $\alpha$ \\
\hline Intrinsic Religiosity Subscale & 3 & .71 \\
Block Dietary Fat Screener & 17 & .84 \\
Economic Adequacy Scale & 7 & .89 \\
Spiritual Well-being Scale & 20 & .81 \\
Healthy Foods and Snacks Barriers Subscale & 15 & .72 \\
Resisting Relapse Subscale & 17 & .78 \\
$\begin{array}{l}\text { Reducing Fat subscale } \\
\text { Self-efficacy for Eating Behaviors Scale }\end{array}$ & 10 & .65 \\
$\begin{array}{l}\text { Family Support for Heart Healthy Eating Habits } \\
\text { Scale }\end{array}$ & 13 & .76 \\
$\begin{array}{l}\text { Encouragement Subscale from the Family Support } \\
\text { for Heart Healthy Eating Habits Scale }\end{array}$ & 6 & .73 \\
$\begin{array}{l}\text { Sabotage Subscale from the Family Support for Heart } \\
\text { Healthy Eating Habits Scale }\end{array}$ & 7 & .76 \\
$\begin{array}{l}\text { Friend Support for Heart Healthy Eating Habits Scale } \\
\text { Positive Comments Subscale from the Friend Support }\end{array}$ & 10 & .67 \\
for Heart Healthy Eating Habits Scale & & .80 \\
$\begin{array}{l}\text { Negative Comments Subscale from the Friend } \\
\text { Support for Heart Healthy Eating Habits Scale }\end{array}$ & 4 & .68 \\
$\begin{array}{l}\text { Perceived Stress Scale } \\
\text { Appraisal of Stressful Situation Subscale from the } \\
\text { General Ethnic Discrimination Scale }\end{array}$ & 17 & .87 \\
\hline
\end{tabular}


Table 6. Cronbach's Alpha Coefficients for if Items Deleted

\begin{tabular}{ll}
\hline Instrument & $\alpha$ if item deleted \\
\hline Reducing Fat Subscale & \\
Item 1 & .55 \\
Item 2 & .61 \\
Item 3 & .66 \\
Item 4 & .64 \\
Item 5 & .65 \\
Item 6 & .60 \\
Item 7 & .67 \\
Item 8 & .64 \\
Item 9 & .60 \\
Item 10 & $*$
\end{tabular}

Friend Support for Heart Healthy

Eating Habits Scale

Item 1

.53

Item 2

.54

Item 3

.60

Item 4

.56

Item 5

.63

Item 6

.66

Item 7

.71

Item 8

.71

Item 9

.66

Item 10

.66

Note. Item 10 in the Reducing Fat subscale had zero variance and was removed by SPSS.

Descriptive statistics, including range, means, medians, and standard deviations of the independent and dependent variables are described in Table 7 . The pilot study sample reported high religious commitment, with scores ranging from 7 to $15(M=13.33, S D=$ 1.76). The dietary fat intake, measured by a score on the BDFS, was categorized in the following manner: very low fat intake (scores from 0 to 7), average fat intake (scores from 8 to 14), quite high fat intake (scores from 15 to 22), and very high fat intake (scores 2 23) (Block et al., 2000). According to the BDFS scoring key (Block et al., 2000), the 
pilot sample's mean DFI score was quite high in fat (scores between 15-22) as shown in Table $7(M=22.01, S D=9.93)$. Using the formulas provided by Block et al. $(2000)$, the percent of daily calories from fat was calculated, which ranged from $22 \%$ to $47 \%(M=$ $34.93, S D=6.01)$. Total fat intake ranged from 44 grams to 145 grams $(M=94.92, S D=$ 24.14) and total saturated fat intake ranged from 6 grams to 43 grams $(M=25.83, S D=$ 8.86). Based on the recommendations by the Mayo Clinic (2013) for a 2,000 calorie-aday diet (total fat $=44-78$ grams/day and saturated fat $=16-22$ grams/day), this sample's mean fat and saturated fat intake were high. 
Table 7. Descriptive Statistics of Pilot Study Variables

\begin{tabular}{|c|c|c|c|c|c|}
\hline Study Variable $(n)$ & $\begin{array}{l}\text { Range of } \\
\text { possible } \\
\text { scores }\end{array}$ & $\begin{array}{l}\text { Range of } \\
\text { sample } \\
\text { scores }\end{array}$ & Mean & Median & $S D$ \\
\hline $\begin{array}{l}\text { Dietary Fat Intake } \\
\text { (31) }\end{array}$ & $0-68$ & $0-42$ & 22.01 & 21.00 & 9.93 \\
\hline $\begin{array}{l}\text { Religious } \\
\text { Commitment (33) }\end{array}$ & $3-15$ & $7-15$ & 13.33 & 14.00 & 1.76 \\
\hline $\begin{array}{l}\text { Economic Adequacy } \\
\text { (31) }\end{array}$ & $7-28$ & $7-28$ & 17.99 & 19.83 & 5.14 \\
\hline Spirituality (29) & $6-120$ & $78-118$ & 101.87 & 104.71 & 10.39 \\
\hline Barriers (28) & $15-60$ & $21-44$ & 34.83 & 34.14 & 5.78 \\
\hline Self-Efficacy (24) & $27-135$ & $91-135$ & 108.49 & 108.47 & 11.02 \\
\hline $\begin{array}{l}\text { Family Social Support } \\
\text { (24) }\end{array}$ & $13-65$ & $29-54$ & 39.92 & 39.00 & 7.11 \\
\hline $\begin{array}{l}\text { Friend Social Support } \\
\text { (24) }\end{array}$ & $10-50$ & $26-44$ & 32.85 & 32.50 & 5.32 \\
\hline Perceived Stress (23) & $0-40$ & $17-28$ & 21.52 & 22.00 & 2.83 \\
\hline $\begin{array}{l}\text { Stress Related to } \\
\text { Racism (23) }\end{array}$ & $17-102$ & $19-73$ & 40.88 & 37.00 & 16.12 \\
\hline
\end{tabular}

Using content analysis, participants' reported barriers to managing dietary fat intake and their feedback regarding the previously mentioned feedback questions were analyzed. Content analysis of the data revealed the following top five categories of barriers that Black emerging adults encountered when managing their dietary fat intake: lack of time ("time constraints on cooking food let alone healthy food"), lack of money ("price of healthy foods can be a little outrageous"), lack of knowledge ("uneducated 
about fat intake"), taste ("high-fat food taste good" and "low-fat food tastes nasty"), and availability ("availability of healthy food is very limited" and fast food is more accessible). Lack of time, taste, and lack of money were the top three reported barriers, in that order.

A majority of the participants $(65 \%)$ reported that the 208 -item survey, which included the demographic questionnaire and feedback questions, was "too long" (Horton, 2013b). One participant wrote, "I almost stopped doing it towards the end." The time it took participants to complete the online survey ranged from 10 to 60 minutes $(M=29$ minutes). One male participant reported that it took two days to complete the survey. Based on the pilot study findings, the proposed online research study was shortened to 113 items.

Of the 20 participants who responded to the feedback question asking asked how can the instructions on the survey be improved, a majority of them (70\%) wrote that nothing needed to be improved (i.e., "written very well and clearly"). Three participants mentioned that the instructions could be shortened, "bit wordy." Two participants wrote that there should be an option to select "not applicable" for some questions. For example, two items on the EAS were not applicable for one participant: does your income allow you to meet your rent or mortgage and does your income allow you to meet baby costs. One participant recommended broadening the "term stressful or define it. Things that make me angry are not necessarily stressful, but maybe it would be helpful to know if I was angry (or sad, or frustrated, etc.)." The participant went on to write that the instructions should specify adult versus childhood experiences. For example, the 
participant shared that she answered questions based on her childhood experiences and was not sure if the investigator wanted, instead, her adult experiences. Lastly, one participant recommended being consistent on pages of the survey with a drop down selection of choices. For example, she said, "If on one page has 'Strongly Agree' at the top of the list, it should remain like this."

Of the 19 participants who responded to the feedback question asking, "were there any questions you did not understand," a majority of them (89\%) wrote that nothing needed to be improved and the survey was "very clear." One participant wrote that the survey was "too long" and one participant wrote that it was "wordy." One participant, who said that the survey was clear, mentioned that there needed to be options added for "not applicable" (i.e., questions about having children but participant did not have children). This participant also recommended distinguishing in what way family opposed the individual's diet (i.e., "you're healthy and they criticize versus you're unhealthy and they criticize"). The participant went on to say, "I found myself wanting to say 'yes they have refused to eat what I cook, but not because it's healthy, because it's unhealthy" (referring to an item on the Family SHHEHS-refused to eat the healthy food I prepared).

Of the 12 participants who responded to the feedback question asking, "were there any questions that made you feel uncomfortable and/or did not like," all of them reported "no." The same 12 participants responded to the feedback question "did you feel distressed by any of questions": 92\% reported "no" and one participant reported "yes". However, the participant reporting "yes" did not write which question made her feel 
distressed and did not request to receive a list of mental health resources.

On the last feedback question, "what other suggestions do you have to improve the wording and clarity of this online survey," seven of the 10 respondents (70\%) reported that nothing needed to be improved. Two participants suggested shortening the survey. One participant suggested adding a "not applicable" option to choose for some questions.

Based on the findings from the pilot study, three changes were made to the current larger study. The Spiritual Well-being Scale was deleted. Its correlation with DFI was smaller $(r=.086)$ compared to religious commitment's (IR subscale from the DUREL) correlation with DFI $(r=.280)$. Whereas the Spiritual Well-being Scale measures individuals' spiritual quality of life, the IR subscale measures the influence of religious beliefs and teachings upon behavior, which is one of the investigator's primary interests. The reducing fat subscale from the Self-Efficacy for Eating Behaviors Scale was deleted. Its correlation with DFI was smaller $(r=.005)$ compared to the resisting relapse subscale's correlation with DFI $(r=.161)$. Its Cronbach's alpha was lower $(\alpha=$ $.65, n=18)$ compared to the resisting relapse subscale $(\alpha=.78, n=12)$. Further, as described in the instrumentation section, the resisting relapse subscale items appear to assess self-efficacy for managing dietary fat intake such as stick to low-fat foods when dining with friends, stick to low-fat foods when at a party, and avoid eating fast food for meals. Lastly, the appraisal of stressful situation subscale, from the General Ethnic Discrimination Scale, was deleted. Although its correlation with DFI was only slightly smaller $(r=-.100)$ compared to the Perceived Stress Scale's correlation with DFI $(\mathrm{r}=$ 
$.104)$, the participants reported less stress related to racism $(M$ score $=40.88$, range of possible scores 19-73) compared to general stress $(M$ score $=21.52$, range of possible scores 17-28). These changes shortened the survey from 208 items to 113 items, which will help lessen the burden of time spent by participants in completing the survey.

\section{Current Study: Instruments and Reliability}

Cronbach's alpha coefficients were used to assess the internal consistency of the eight instruments used in this study: Block Dietary Fat Screener, Intrinsic Religiosity Subscale, Economic Adequacy Scale, Healthy Foods and Snacks Barriers Subscale, Resisting Relapse Subscale, Family Support for Heart Healthy Eating Habits Scale, Friend Support for Heart Healthy Eating Habits Scale, and Perceived Stress Scale. The instruments' reliability results are reported in Table 15. A Cronbach's alpha of .70 or greater suggests acceptable internal consistency for an instrument (Fitzpatrick, 1998). All but two of the instruments met this criterion: Family Support for Heart Healthy Eating Habits Scale $(\alpha=.69)$ and Friend Support for Heart Healthy Eating Habits Scale ( $\alpha=$ .63). Sallis et al. (1987) reported internal consistencies ranging from .61 to .91 for both of these scales. For this current study reliability tests were conducted on the subscales for each instrument. The Family Support for Heart Healthy Eating Habits Scale is made of two subscales: encouragement (six items, $\alpha=.88$ ) and sabotage (seven items, $\alpha=.77$ ). The Friend Support for Heart Healthy Eating Habits Scale is made of two subscales: positive comments (six items, $\alpha=.92$ ) and negative comments (four items, $\alpha=.86$ ). To determine the possible influence of the subscales on the overall results of the study. Total subscale scores were computed, and then their correlations with the outcome variable 
(DFI) were examined. There were no significant relationships among the subscales and DFI. Since there were no significant relationships, they were not entered into the regression models for this study. Similar results was found for the total score for both the Family Support for Heart Healthy Eating Habits Scale and Friend Support for Heart Healthy Eating Habits Scale, which were not entered into the regression models for this study.

\section{DATA ANALYSIS}

Once all the participants had completed the online survey, the researcher downloaded the data into an Excel file, which was then transferred to a SPSS file for data analysis. The data was then deleted from the online server. Data analysis was conducted on a password-protected computer. The level of statistical significance for all of the research questions was set at $p<.05$. Descriptive statistics were used to describe characteristics of the study's sample (i.e., frequencies, percents, means, and standard deviations). Correlation coefficients were used to examine the relationships between the independent variables and dependent variable. Multiple regression was used to determine the significant predictors for DFI (Keith, 2006).

\section{Data Cleaning}

In order to ensure accurate data analysis, the investigator cleaned the data, which entailed assessing for errors (i.e., data entry errors for paper version of surveys), missing data, violations of multiple regression assumptions, and multicollinearity. The online version of the survey was downloaded directly into an Excel file, which decreased the 
risk for human error in data entry. To assess for errors in data entry for the paper version of the survey, a colleague checked each item entry in the Excel file for all the paper surveys $(n=49)$. Errors that were identified by the colleague were corrected by the investigator $($ percent error $=.37 \%$ ). After the paper survey data entries were verified for accuracy, the online and paper survey data were combined into one Excel file. Then the combined Excel file was transferred to a SPSS file for data analysis. Using SPSS (version 21), univariate descriptive statistics were then examined for out-of-range values, reasonable means and standard deviations, and outliers.

\section{Missing Data}

Although participants were encouraged to answer every item in the survey, it was inevitable there would be some missing data. Therefore, the investigator followed the scoring instrument guidelines provided by the instrument developer. In addition, the amount and distribution of missing data was evaluated (Table 13). Using the Missing Value Analysis in SPSS (version 21), the pattern of missing values in the data was assessed. In addition, multiple imputations within SPSS were used to impute cases in which 5\% or more data were missing.

\section{Assumptions}

After selecting a method for addressing missing data, it was ensured that the assumptions for Pearson's $r$, Chi-square, point biserial, and multiple regression were met prior to conducting these analyses. The assumptions for Pearson's $r$ are the following: there is a representative sample of the population, normal distribution of the scores, equal variability in the distribution of $Y$ scores (homoscedasticity), and a linear relationship 
between $X$ and $Y$ (straight line) (Munro, 2005). The assumptions for Chi-square are the following: the data are frequency data, there is an adequate sample size, the created categories are independent from each other, and the created categories have a theoretical basis (Munro, 2005).

The assumptions for multiple regression are the following:

- Linear relationship between the dependent variable (DI) and predictor variables (linearity)

- Independence of errors

- Constant residual variance around the regression line (homoscedasticity)

- Normal distribution of errors (Field, 2005; Keith, 2006; Warner, 2013).

The next sections describe how violations of the above-mentioned assumptions were determined.

Linearity. The investigator assessed if the relationships in the model were linear. To determine linearity, bivariate scatter plots were examined to assess for nonlinearity (Keith, 2006). Inspection of the scatter plots did not show any curvilinear relationships. The scatter plots for perceived barriers and perceived self-efficacy appeared to have a linear relationship with the outcome variable (DFI). However, the scatter plots for BMI, religious commitment, income, perceived family support, perceived friend support, and stress appeared to show no relationship with the outcome variable (Appendix P). Since gender is a dichotomous variable, a scatter plot was not graphed. The scatter plots that excluded outliers were also inspected, which were similar results. 
Independence of Errors. The Durbin-Watson test and boxplots of residuals were used to evaluate non-independence of errors (Keith, 2006).

Homoscedasticity. To determine violation of homoscedasticity, scatterplots of residuals with predictor variables were examined (Keith, 2006).

Normal distribution of errors. To determine violation of normal distribution of errors, the results of normality tests, bar graph of the residuals, and q-q plot of residuals were examined (Field, 2005; Keith, 2006).

Multicollinearity. Lastly, multicollinearity is another possible threat to the validity of this study. The investigator examined whether or not the predictor variables were highly correlated in the correlation matrix, which is any correlation > .90 (Field, 2005; Warner, 2013). In addition, using SPSS, collinearity diagnostics such as variance inflation factor (VIF), tolerance statistic (1/VIF), and index of tolerance were ran (Field, 2005).

\section{Research Questions}

After the data were assessed for accuracy and quality (i.e., addressed missing data, violations of multiple regression assumptions, and multicollinearity), the following research questions were addressed:

\section{Research Question 1}

What are the relationships among the individual characteristics (gender, BMI, income adequacy, and religious commitment), behavior-specific cognitions and affect (perceived barriers for healthy eating, perceived self-efficacy related to dietary fat 
intake, perceived family social support, perceived friend social support, and perceived generalized stress), and dietary fat intake among Black emerging adults?

Pearson correlations (Pearson's $r$ ) were used to examine relationships among interval level data (i.e., BMI and dietary fat intake score). Chi-square analysis $\left(\chi^{2}\right)$ was used to examine relationships among nominal level data (i.e., education level). Point biserial correlations were used to examine relationships among dichotomous and interval data (i.e., gender and dietary fat intake score) (Huck, 2008).

\section{Research Question 2}

What are the significant predictors for dietary fat intake among the independent variables of individual characteristics (gender, BMI, income adequacy, and religious commitment) and behavior-specific cognitions and affect (perceived barriers for healthy eating, perceived self-efficacy related to dietary fat intake, perceived family social support, perceived friend social support, and perceived generalized stress) for Black emerging adults?

Based on the results of Research Question 1, variables with significant correlations with DFI were entered into a model for Research Question 2. The following model was tested in the simultaneous multiple regression analysis: $Y^{\prime}=a+b_{1} X_{1}+b_{2} X_{2}$ $+\ldots+b_{k} X_{k} . Y^{\prime}$ represents the predicted $Y$. Y represents the dependent variable, dietary fat intake. $\mathrm{X}_{1} \ldots$ to $\mathrm{X}_{\mathrm{k}}$ represents the predictor variables. $\mathrm{Y}$ was regressed on the predictor variables. The below equation includes all of the variables that could be possibly tested: 
Dietary fat intake $=a+b_{1}($ Gender $)+b_{2}(B M I)+b_{3}($ Income adequacy $)+$ $\mathrm{b}_{4}($ Religious commitment $)+\mathrm{b}_{5}$ (Perceived barriers for healthy eating $)+$ $\mathrm{b}_{6}($ Perceived self-efficacy related to dietary fat intake $)+b_{7}($ Perceived family social support $)+b_{8}($ Perceived friend social support $)+b_{9}($ Perceived generalized stress) (Warner, 2013).

Simultaneous multiple regression was used to determine the significant predictors for dietary fat intake (Keith, 2006). Using SPSS, the predictor variables were entered into one block (gender, BMI, income adequacy, religious commitment, perceived barriers for healthy eating, perceived self-efficacy related to dietary fat intake, perceived family social support, perceived friend social support, and perceived generalized stress). The $R^{2}$ change and $F$ and $p$ values were assessed for significance in predicting DFI. Lastly, the resulting coefficients table was evaluated to determine significant predictor variables for DFI (unstandardized coefficients $[b]$ and associated $t$ values) (Keith, 2006; Warner, 2013).

\section{Research Question 3}

After controlling for individual characteristics (gender, BMI, income adequacy, and religious commitment), what are the significant predictors for dietary fat intake among Black emerging adults?

In order to take into account the variation explained by gender, BMI, income adequacy, and religious commitment, hierarchical regression was used to answer the above question. Dietary fat intake was regressed on the individual characteristics 
variables and behavior-specific cognitions and affect variables. Based on the theoretical framework, hierarchical regression was used and the individual characteristics (gender, BMI, income adequacy, and religious commitment) were entered in the first block, followed by the behavior-specific cognitions and affect variables (perceived barriers for healthy eating, perceived self-efficacy related to dietary fat intake, perceived family social support, perceived friend social support, and perceived generalized stress in the second block (Keith, 2006).

The models (Model 1 and Model 2) were compared by examining the change in $R^{2}$. The $R^{2}$ change and $F$ and $p$ values were assessed for significance in predicting DFI. Lastly, the resulting coefficients table for Model 1 and Model 2 were evaluated to determine significant predictor variables for DFI (unstandardized coefficients $[b]$ and associated $t$ values) (Keith, 2006).

\section{Research Question 4}

Do perceived family social support and perceived friend social support moderate the effects of individual characteristics (gender, BMI, income adequacy, and religious commitment) on dietary fat intake?

It is appropriate to test for the influence of moderators when you think a third variable influences the strength of the relationship between the independent variable and a dependent variable (Baron \& Kenny, 1986) (refer to Figure 2). Thus, the ability of the independent variables (individual characteristics: gender, BMI, income adequacy, and religious commitment) to predict the dependent variable (dietary fat intake) may depend 
on varying levels of the moderator variables (perceived family social support and perceived friend social support) (Baron \& Kenny, 1986). When the moderator variable influences the relationship between the independent and dependent variable it is also called an interaction ( $X_{1}$ and $X_{2}$ interact as predictors of Y) (Warner, 2013).

In order to test for the moderator effect of perceived family social support and perceived friend social support, hierarchical multiple regression analysis was used (Bennett, 2000). An interaction term, which is the product of the predictor variable and moderator variable, was created to test for the moderator effect of perceived family social support. Prior to creating the interaction term, all of the predictor variables were centered (BMI, income adequacy, religious commitment, and perceived family social support) (Keith, 2006; Warner, 2013). Centering the variables reduces "the correlation between the product term and the $X_{1}, X_{2}$ scores, so that the effects of the $X_{1}$ and $X_{2}$ predictors are distinguishable from the interaction" (Warner, 2013, p. 632). Centering is accomplished in SPSS by "subtracting the sample mean from the scores on each predictor" variable (Warner, 2013, p. 632). This computation results "in a new variable with a mean of zero and a standard deviation equal to the original standard deviation" (Keith, 2006, p. 133).

Figure 2 depicts a moderator effect of perceived family social support and perceived friend social support. As per standard steps described by Aguinis (2004), Keith (2006), and Miles and Shevlin (2001), hierarchical analyses were used to test for the moderator effect of perceived family social support. The results of Model 2 will determine if a moderator effect exists after controlling for gender, BMI, income adequacy, religious commitment, and perceived family social support. If the second 
model results in a statistically significant difference in the Adjusted $R^{2}(F$ and $p$ values of $\Delta R^{2}$ ), then perceived family social support moderates the relationship between each independent variable (individual characteristics: gender, BMI, income adequacy, and religious commitment) and the dependent variable (dietary fat intake). In other words, perceived family social support and the individual characteristics interact in their effect on DFI. These same hierarchical analyses were used to test for the moderator effect of perceived friend social support.

Figure 2. Theoretical Frameworks for Moderators for Dietary Fat Intake

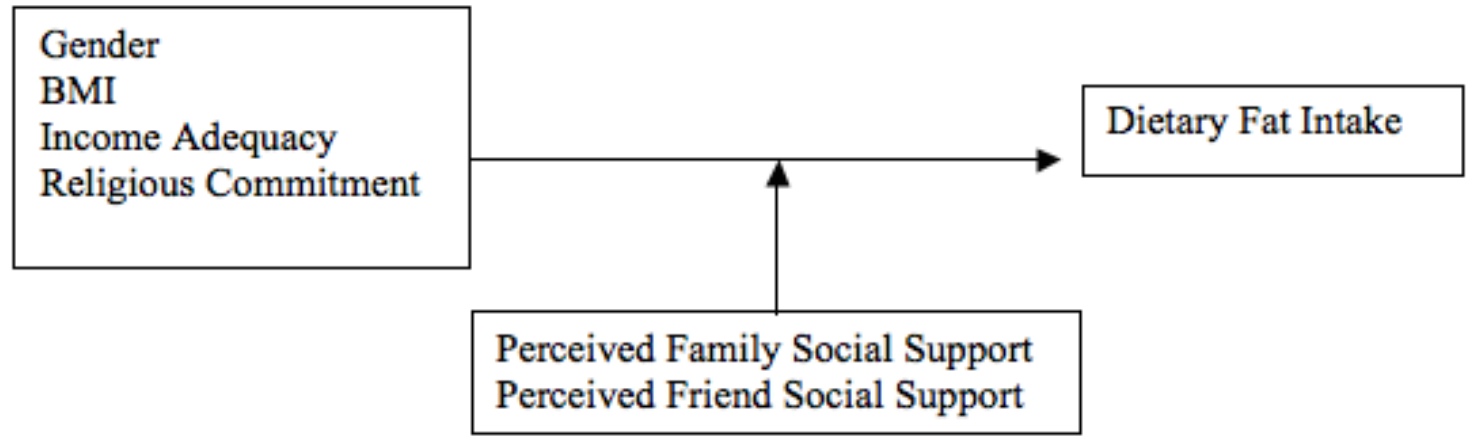

\section{Research Question 5}

Does religious commitment moderate the effect of perceived generalized stress on dietary fat intake?

Figure 3 depicts a moderator effect of religious commitment. Similar to Question 4, hierarchical analyses to test for the moderator effect of religious commitment in Question 5 was used; except the dependent variable (DFI) was regressed on the 
interaction term (perceived generalized stress) and moderator variable (religious commitment).

Figure 3. Theoretical Frameworks for Moderators for Dietary Fat Intake

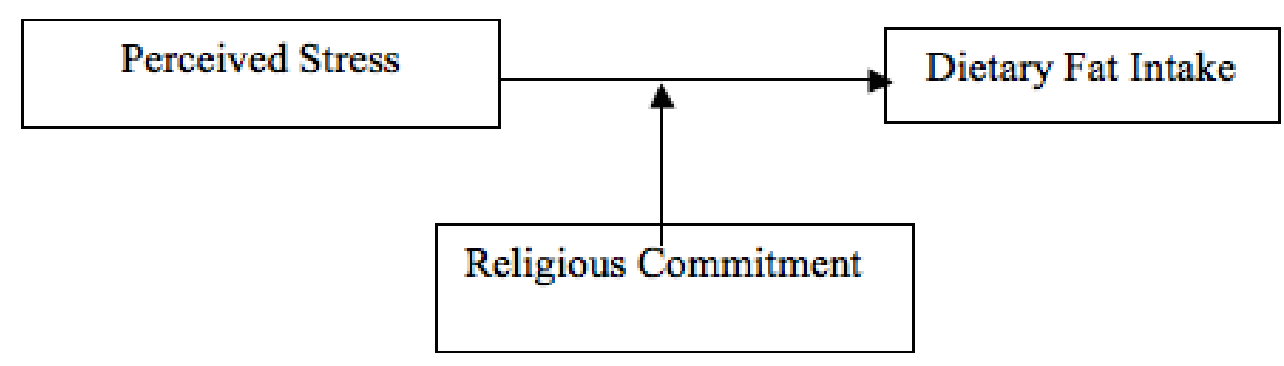

\section{Research Question 6}

Does perceived generalized stress mediate the effect of religious commitment on dietary fat intake?

A mediator explains the relationship between an independent variable and dependent variable (Baron \& Kenny, 1986; Bennett, 2000). In this case, it is assumed that perceived generalized stress explains how religious commitment relates to dietary fat intake. Before a mediator effect can be tested, there has to be a significant direct effect between the independent variable (religious commitment) and the dependent variable (dietary fat intake) (Baron \& Kenny, 1986; Bennett, 2000; Warner, 2013). If the following conditions are met, then a mediator effect exits:

- Variations in the independent variable (religious commitment) predict variations in the mediator variable (perceived generalized stress). 
- Variations in the mediator variable (perceived generalized stress) predict variations in the outcome variable (dietary fat intake).

- When the relationships in previous two conditions are controlled in the model, the direct relationship between the independent variable (religious commitment) and the outcome variable (dietary fat intake) is nonsignificant (Baron \& Kenny, 1986; Bennett, 2000).

If the relationship between religious commitment and dietary fat intake reduces to zero, then perceived generalized stress is a full mediator (Baron \& Kenny, 1986; Bennett, 2000). Partial mediation is evident if the relationship between religious commitment and dietary fat intake nears zero (Baron \& Kenny, 1986; Bennett, 2000). A depiction of this potential mediating effect is shown in Figure 4.

Figure 4. Theoretical Framework for Mediator for Dietary Fat Intake

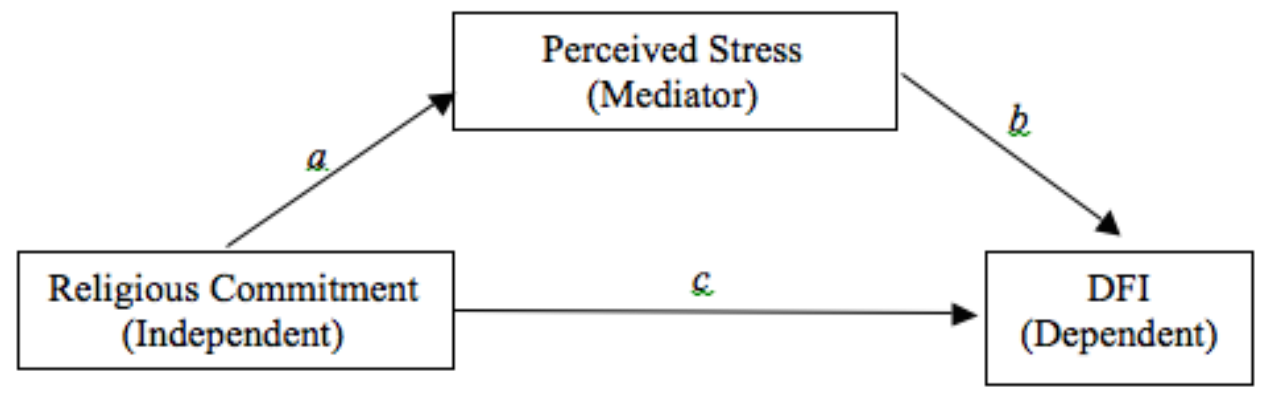

It is assumed that perceived generalized stress mediates the relationship between religious commitment and dietary fat intake, which is represented in Figure 4. To test whether perceived generalized stress mediates the effect of religious commitment on dietary fat intake, a series of regression models will be used, which first must meet two assumptions: 1) there is not any measurement error in the mediator and 2) the dependent 
variable does not cause the mediator variable (Baron \& Kenny, 1986). Following the standard steps described by Warner (2013), testing for mediation will be conducted, along with performing the Sobel test. The Sobel test examines the indirect effects of the independent variable (religious commitment) on the dependent variable (dietary fat intake) through the mediator variable (perceived generalized stress), in which the null hypothesis is $\mathrm{H}_{\mathrm{o}}$ : $a b=0$ (Baron \& Kenny, 1986; Preacher \& Hayes, 2004; Warner, 2013). This test will be used to assess the significance of mediation, which involves examining "the product of the $a, b$ coefficients for the mediated path" (Warner, 2013, p. 656). The standardized and unstandardized coefficients of the Sobel test will be examined to determine the total effects that religious commitment has on dietary fat intake, and whether it is influenced by perceived generalized stress. If the indirect effect is significant, then the relationship between religious commitment and dietary fat intake is indirectly mediated by perceived generalized stress.

\section{PROTECTION OF HUMAN SUBJECTS}

Permission to conduct this study was obtained and approved by the University of Texas at Austin's IRB and investigator's PhD dissertation committee. The participants recruited for the study received a cover letter explaining the purpose of the study, content of the questions in the survey, risks and benefits of participating in the study, how they will be respected as human subjects, and how their privacy and confidentiality will be protected (see Appendix A). When participants accessed the web link for the survey, a consent form to participate in Internet research was on the opening page (see Appendix 
B). If they agreed to participate, they clicked the button next to the statement "Yes, I read and understand the consent. I agree to participate in this study." If they did not wish to participate in the study, they could exit the Internet browser or click the button next to the statement "I do not want to participate in this study," which then exited them out of the survey site. An option was available for participants to click in order to print a copy of the consent.

There were no foreseeable risks in participating in this proposed study. Participants, who felt burdened by any of the questions in the survey, could click an option button on the last page of the online survey: Yes, send me a list of mental health resources. A list of mental health resources were sent to the email provided by the participant, if requested. A copy of the email message and list of resources sent via email is provided in Appendix I.

The participants' survey responses were stored on an encrypted server. Participants could decline to answer any question and withdraw from the study at any time. To maintain confidentiality, after all the participants completed the survey, the deidentified data (removal of email addresses) were transferred into a data file on a password-protected computer, and data was deleted from the online server. The web link for the survey was set up to close once 272 participants had completed the survey, including a closure explanation message for visitors to the web link. 


\section{SUMMARY}

This chapter described the research design, sample, sample size, and power analysis used in this study. A cross-sectional, descriptive correlational research design was used in a sample of Black emerging adults. A description of the sample $(N=272)$, recruiting process, and inclusion criteria was provided. A detailed explanation of the measurement tools, including their validity and reliability, and procedures for data collection, data cleaning, and handling missing data were presented. Using SPSS, version 21 , data collected was analyzed to answer the previously mentioned research questions. Lastly, ethical concerns and the protection of human subjects were reviewed in this chapter. 


\section{Chapter 4: Results}

This chapter describes statistical results for data cleaning and the results of data analyses relevant to the research questions. The first section of data analyses provides demographic characteristics for the sample of Black emerging adults. The second section provides descriptive analyses of the variables, including instrument reliability, used in this study. And the third section is findings for each research question.

\section{Testing Assumptions}

Any violations of the assumptions for multiple regression were assessed (nonlinearity, independence of errors, normal distribution of errors, and multicollinearity). Violation of nonlinearity and homoscedasticity was determined by examination of bivariate scatter plots (Keith, 2006). Independence of errors violation was determined by the Durbin-Watson test. Normal distribution of errors violation was determined by normality tests. Multicollinearity violation was determined by variance inflation factor (VIF) and tolerance statistics.

Scatter plots for all variables were assessed for nonlinearity, and transformations were conducted as needed, which are described later. Independence of error was not violated based on examination of the q-q plots and the Durbin-Watson test results, which were $>1$ and $<3$ (Field, 2005). Based on the scatterplots of residuals with predictor variables, no violation of homoscedasticity was determined. As for multicollinearity, there were no highly correlated variables (>.90) in the correlation matrix. Based on the criteria reported by Field (2005), the VIF and tolerance statistics did not indicate any 
concern for multicollinearity. There were no out-of-range values. However, one independent variable had a minimum value that equaled zero: DFI (participant \#524). This participant chose the same answer for several questions. Due to concerns about validity of the DFI data for participant \#524, this participant was excluded when data analysis was conducted.

Based on the univariate descriptive statistics, all of the variables had reasonable means and standard deviations. Since the sample size was relatively large $(>200)$, the skewness and kurtosis values were converted to $z$-sores. Then the investigator assessed whether or not the $z$-score values for each variable were $>3.29$ (absolute values above 3.29 are significant at $p<.001$ ) (Field, 2005). The results of variables with significant skewness and kurtosis are reported in Table 8. The significant positive skewed variables were BMI, DFI, and perceived friend support. Gender and religious commitment were found to have significant negative skewness. Body mass index and perceived friend support had significant positive kurtosis findings. 
Table 8. Skewness and Kurtosis Results

\begin{tabular}{|c|c|c|c|c|c|c|}
\hline Variable & Skewness & $\begin{array}{r}\text { Standard } \\
\text { Error of } \\
\text { Skewness }\end{array}$ & Zskewness & Kurtosis & $\begin{array}{l}\text { Standard } \\
\text { Error of } \\
\text { Kurtosis }\end{array}$ & Zkurtosis \\
\hline Gender & -1.239 & .150 & $-8.26^{*}$ & -.468 & .298 & -1.57 \\
\hline BMI & 1.023 & .153 & $6.69^{*}$ & 1.195 & .304 & $3.93^{*}$ \\
\hline DFI & .689 & .153 & $4.50^{*}$ & .976 & .306 & 3.19 \\
\hline $\begin{array}{l}\text { Religious } \\
\text { Commitment }\end{array}$ & -.1 .359 & .154 & $-8.82^{*}$ & 1.002 & .306 & 3.27 \\
\hline Income & -.089 & .156 & -.57 & -.350 & .310 & -1.13 \\
\hline $\begin{array}{l}\text { Perceived } \\
\text { Barriers }\end{array}$ & .431 & .158 & 2.73 & .491 & .314 & 1.56 \\
\hline $\begin{array}{l}\text { Perceived Self- } \\
\text { efficacy }\end{array}$ & -.502 & .159 & -3.16 & -.035 & .318 & -.11 \\
\hline $\begin{array}{l}\text { Perceived } \\
\text { Family Support }\end{array}$ & .230 & .162 & 1.42 & .351 & .322 & 1.08 \\
\hline $\begin{array}{l}\text { Perceived Friend } \\
\text { Support }\end{array}$ & .631 & .164 & $3.85^{*}$ & 1.162 & .326 & $3.56^{*}$ \\
\hline Stress & -.003 & .164 & .018 & -.099 & .326 & -.304 \\
\hline
\end{tabular}

* $z$-score value significant at $p<.001$

Since large sample sizes may have small standard errors, they may have significant values due to small deviations from normality (Field, 2005). Therefore, it is important to examine the distribution visually (i.e., histograms) (Field, 2005; Kim, 2013). All of the histograms appeared to mirror the findings in Table 8, except perceived friend support appeared to have a normal distribution (Appendix M).

In addition, tests of normality were conducted for each variable in this study, except gender since it is a dichotomous variable. The results are reported in Table 9. A non-significant $\mathrm{p}$ value $(p>.05)$ means the sample distribution is not significantly different from a normal distribution (Field, 2005). Based on the tests of normality in Table 9, all of the variables were significant for either or both the Kolmogorov-Smirnov 
and Shapiro-Wilk tests, which means in general there was not a normal distribution of scores among the variables in the study.

Table 9. Tests of Normality

\begin{tabular}{lcccccc}
\hline Variable & \multicolumn{3}{c}{$\begin{array}{c}\text { Kolmogorov- } \\
\text { Smirnov }\end{array}$} & \multicolumn{3}{c}{ Shapiro-Wilk } \\
\cline { 2 - 7 } & Statistic & df & Sig. & \multicolumn{1}{c}{ Statistic } & df & Sig. \\
\hline BMI & .093 & 255 & .000 & .939 & 255 & .000 \\
DFI & .066 & 252 & .010 & .970 & 252 & .000 \\
Religious Commitment & .208 & 251 & .000 & .801 & 251 & .000 \\
Income & .113 & 245 & .000 & .965 & 245 & .000 \\
Perceived Barriers & .055 & 238 & .079 & .984 & 238 & .010 \\
Perceived Self-efficacy & .060 & 233 & .041 & .969 & 233 & .000 \\
Perceived Family Support & .076 & 226 & .003 & .990 & 226 & .103 \\
Perceived Friend Support & .110 & 221 & .000 & .953 & 221 & .000 \\
Stress & .058 & 221 & .066 & .994 & 221 & .475 \\
\hline
\end{tabular}

Next all values for each variable were changed to $z$-scores to determine outliers that were greater than three standard deviations from the mean (Field, 2005). The only variables that did not have outliers greater than three standard deviations from the mean were religious commitment and income.

Table 10 describes which participants were identified as outliers for each variable. 
Table 10. Outliers $>3$ Standard Deviations

\begin{tabular}{lll}
\hline Variable & Participant \# & Version of Survey \\
\hline BMI & 200 & Online \\
& 210 & Online \\
& 235 & Online \\
& 471 & Online \\
DFI & 318 & Online \\
& 466 & Online \\
& 501 & Paper \\
& 526 & Paper \\
Perceived Barriers & 207 & Online \\
& 466 & Online \\
& 525 & Paper \\
Perceived Self-efficacy & 207 & Online \\
Perceived Family Support & 210 & Online \\
& 496 & Paper \\
& 513 & Paper \\
Perceived Friend Support & 254 & Online \\
& 496 & Paper \\
& 513 & Paper \\
Stress & & \\
\hline
\end{tabular}

Univariate descriptive statistics were repeated with the outliers identified in 
Table 10 excluded, including participant \#524 responses for DFI. Again, the skewness and kurtosis values for all the study's variables, except gender, were converted to $z$-sores. Then the $z$-score values for each variable were assessed for whether or not they were $>3.29$ (absolute values above 3.29 are significant at $p<.001)$ (Field, 2005). The skewness and kurtosis results are reported in Table 11. In general, there was improvement in the distribution of scores for all the variables when the identified outliers in

Table 10 and participant \#524 were excluded compared to the results in Table 8. Unlike the significant findings in Table 11, all of the histograms appeared to have normal distributions, except BMI, religious commitment, and perceived friend support. Body mass index and perceived friend support had moderate positive skewness and religious commitment had substantial negative skewness. Therefore, the identified outliers in 
Table 10 were excluded in subsequent data analyses. Histograms and Q-Q plots with and without the outliers can be found in Appendix M and Appendix N.

Table 11. Repeated Skewness and Kurtosis Results (Outliers Not Included)

\begin{tabular}{|c|c|c|c|c|c|c|}
\hline Variable & Skewness & $\begin{array}{r}\text { Standard } \\
\text { Error of } \\
\text { Skewness }\end{array}$ & Zskewness & Kurtosis & $\begin{array}{r}\text { Standard } \\
\text { Error of } \\
\text { Kurtosis }\end{array}$ & Zkurtosis \\
\hline BMI & .713 & .156 & $4.57^{*}$ & .261 & .310 & .84 \\
\hline DFI & .273 & .158 & 1.73 & -.470 & .315 & -1.49 \\
\hline $\begin{array}{l}\text { Religious } \\
\text { Commitment }\end{array}$ & -1.308 & . 158 & $-8.28^{*}$ & .824 & .316 & 2.61 \\
\hline Income & -.088 & .160 & -.55 & -.373 & .320 & -1.17 \\
\hline $\begin{array}{l}\text { Perceived } \\
\text { Barriers }\end{array}$ & .188 & .163 & 1.15 & -.061 & .324 & -.19 \\
\hline $\begin{array}{l}\text { Perceived Self- } \\
\text { efficacy }\end{array}$ & -.421 & . 165 & -2.55 & -.280 & .328 & -.85 \\
\hline $\begin{array}{l}\text { Perceived } \\
\text { Family Support }\end{array}$ & .189 & .167 & 1.13 & -.266 & .333 & -.80 \\
\hline $\begin{array}{l}\text { Perceived Friend } \\
\text { Support }\end{array}$ & 651 & . 169 & $3.85^{*}$ & .549 & .337 & 1.63 \\
\hline Stress & .006 & .169 & .04 & -.093 & .337 & -.28 \\
\hline
\end{tabular}

Since the BMI, religious commitment, and perceived friend support variables remained significant for skewness, these three variables were transformed. Body mass index and perceived friend support were transformed using a square root transformation, which in SPSS is the following: NEWX=SQRT(X) (Tabachnick \& Fidell, 2013). 
Religious commitment was transformed using a reflect and logarithm transformation, which in SPSS is the following: NEWX=LG10(K-X) (Tabachnick \& Fidell, 2013). Then tests of normality on the transformed variables were conducted (BMI, religious commitment, and perceived friend support), excluding outliers, which the results are reported in Table 12. A non-significant $p$ value $(p>.05)$ means the sample distribution is not significantly different from a normal distribution (Field, 2005). Based on the tests of normality in Table 12, the transformed BMI, religious commitment, and perceived friend support variables remain significant for either or both the Kolmogorov-Smirnov and Shapiro-Wilk tests. However, the square root transformation of BMI and perceived friend support reduced the skewness of those variables, which is the most symmetric, nearnormal distribution. In addition, the BMI and perceived friend support histograms and QQ plots showed some improvement in the shape of their distribution of scores (Appendix N). Based on the Q-Q plot, there was some improvement in the distribution transformed religious commitment scores; but the histogram did not appear to show improvement (Appendix $\mathrm{M}$ and Appendix $\mathrm{N}$ ). The transformed BMI, religious commitment, and perceived friend support variables were used during subsequent data analysis.

Table 12. Tests of Normality for Transformed Variables (Outliers Not Included)

\begin{tabular}{|c|c|c|c|c|c|c|}
\hline \multirow[t]{2}{*}{ Variable } & \multicolumn{3}{|c|}{ Kolmogorov-Smirnov } & \multicolumn{3}{|c|}{ Shapiro-Wilk } \\
\hline & Stati & & df Sig. & Statistic & $\mathrm{df}$ & Sig. \\
\hline BMI Square Root & .056 & 244 & .065 & .980 & 244 & .001 \\
\hline $\begin{array}{l}\text { Religious Commitment } \\
\text { Reflect/Log }\end{array}$ & .215 & 236 & .000 & .886 & 236 & .000 \\
\hline $\begin{array}{l}\text { Perceived Friend Support } \\
\text { Square Root }\end{array}$ & .094 & 207 & .000 & .971 & 207 & .000 \\
\hline
\end{tabular}




\section{Missing Data}

Based on the Little's MCAR test result, the data for this study were missing completely at random (Chi square $=101.08, \mathrm{DF}=82, p=.08)$. The investigator then repeated the Missing Value Analysis excluding the outliers identified in

Table 10 and participant \#524. Based on the Little's MCAR test result, the data

for this study remained missing completely at random (Chi square $=6.71, \mathrm{DF}=8, p=$ .569). Upon visual inspection of Table 13, it appears that the order of the instruments may have slightly influenced data that would be missing. The findings in Table 13 were determined by using analyze pattern with multiple imputations in SPSS. Paired variables, the independent variable in relation to the dependent variable (i.e., gender and DFI), were analyzed, which is why DFI is not listed in Table 13. However, according to the univariate missing value analysis performed in SPSS, DFI had $5.6 \%$ missing data $(n=$ 235). Multiple imputations were used to determine if the missing data influenced the data analysis findings. If the missing data did not influence the findings, then pairwise deletion would be used for the data analysis. 
Table 13. Missing Data

\begin{tabular}{lll}
\hline Variable & Complete Cases & \% Cases Missing \\
\hline Gender & 236 & 5.98 \\
BMI & 232 & 7.57 \\
Religious Commitment & 236 & 5.98 \\
Income & 230 & 8.37 \\
Perceived Barriers & 223 & 11.16 \\
Perceived Self-efficacy & 218 & 13.15 \\
Perceived Family Support & 211 & 15.94 \\
Perceived Friend Support & 207 & 17.53 \\
Stress & 207 & 17.53 \\
\hline
\end{tabular}

\section{Imputations for Missing Data}

Mean score computations were used to obtain the total score for instruments used in the study. Therefore, missing data for the variables examined in this study are truly missing. To determine whether or not missing data influenced the results, data analysis using multiple imputations in SPSS was conducted. The results are reported after each research question discussed later in this chapter.

\section{Mahalanobis Distance and Cook's Distance}

Based on the Cook's distance results $(<1)$ for all of the regressions conducted for this study, there were no identified cases that had an undue influence on the models. According to Field (2005), values $<1$ are acceptable. None of the cases exceeded the Mahalanobis distance criterion, which suggest that there are no influential cases within the data (Field, 2005).

\section{DEMOGRAPHICS OF THE SAMPLE}

A total of 528 Black emerging adults participated in the study. Of the 528 participants, 262 were excluded from analyses due to ineligibility. Of the 262 participants 
excluded from the study, 44 did not meet the inclusion criteria: live in the U.S., read and speak English, self-identify as Black, age 18 to 25 years, and not pregnant. The other 218 participants completed the online version of the survey; they were excluded because their survey was disqualified for the following one or more reasons: a) it was completed outside of the United States; b) the reported height and weight were not logically realistic; c) more than one survey was completed from the same IP address and/or latitude and longitude area; and d) the time period to complete the survey was not logically realistic (i.e., completed survey in less than five minutes). Of the remaining 266 participants, 49 completed the paper version and 217 completed the online version of the survey. The identified outliers in

Table 10 and participant \#524 were excluded from data analysis (seven paper and eight online versions, 15 total), which made the final sample size 251 participants. Demographic characteristics of the sample are displayed in Table 14.

A majority of the participants lived in Texas (64.78\%) and Louisiana (28.34\%). Of the participants, for which their recruitment site was identified $(n=169)$, about $76 \%$ of the participants were recruited through colleges/universities. Forty-six (18.3\%) of the self-identified Black emerging adult sample also considered themselves to be multiethnic. 
As seen in Table 14, the average age of the participants was 20.49 years $(S D=2.01)$ and ranged from 18 to 25 years $(N=251)$. Of those participants who reported their gender $(n$ =250), there were $56(22.4 \%)$ males and $194(77.6 \%)$ females. A majority of the sample was single (94\%), lived with their parents $(32.7 \%)$ or a roommate $(44.4 \%)$, and reported having no children $(94.8 \%)$. Most of the participants reported having completed some college $(54.4 \%)$ and being either unemployed (30.6\%) or part-time employed $(36.3 \%)$. Most of the participants reported weighing themselves once a month (36.1\%) or every six months $(26.9 \%)$; and they reported that they considered their weight "just about right" (43.4\%) or "somewhat overweight" (38.6\%). 
Table 14. Demographic Characteristics of Sample

\begin{tabular}{|c|c|c|c|c|}
\hline $\begin{array}{l}\text { Demographic } \\
\text { Characteristic } \\
\end{array}$ & Categories & $\%(\mathrm{n})$ & Mean (SD) & Range \\
\hline Age & & $(251)$ & $20.49(2.01)$ & $18-25$ \\
\hline \multirow[t]{2}{*}{ Multiethnic } & Yes & $18.30(46)$ & & \\
\hline & No & $81.70(205)$ & & \\
\hline \multirow[t]{2}{*}{ Gender } & Female & $77.6(194)$ & & \\
\hline & Male & $22.4(56)$ & & \\
\hline \multirow[t]{6}{*}{ Education Level } & $11^{\text {th }}$ Grade & $.80(2)$ & & \\
\hline & High School/GED & $20.6(51)$ & & \\
\hline & Some College & $54.4(135)$ & & \\
\hline & Associate Degree & $5.6(14)$ & & \\
\hline & Bachelor's Degree & $16.1(40)$ & & \\
\hline & Graduate Degree & $2.4(6)$ & & \\
\hline \multirow[t]{3}{*}{ Marital Status } & Single & $94(233)$ & & \\
\hline & $\begin{array}{l}\text { Not married, living } \\
\text { with partner }\end{array}$ & $3.6(9)$ & & \\
\hline & Married & $2.4(6)$ & & \\
\hline \multirow[t]{2}{*}{ Has Children } & Yes & $5.2(13)$ & & \\
\hline & No & $94.8(235)$ & & \\
\hline \multirow{5}{*}{ Living Arrangement } & Lives with roommate & $44.4(110)$ & & \\
\hline & Lives with parents & $32.7(81)$ & & \\
\hline & Lives Alone & $12.5(31)$ & & \\
\hline & $\begin{array}{l}\text { Lives with } \\
\text { spouse/partner }\end{array}$ & $4.4(11)$ & & \\
\hline & Other & $6(15)$ & & \\
\hline \multirow[t]{5}{*}{ Employment Status } & Full-time & $17.7(44)$ & & \\
\hline & Part-time & $36.3(90)$ & & \\
\hline & Self-employed & $2.4(6)$ & & \\
\hline & Unemployed & $30.6(76)$ & & \\
\hline & Other & $12.9(32)$ & & \\
\hline
\end{tabular}

Note. Because of missing data, total participants of some demographics do not equal 251. Percentages may not add up to $100 \%$ due to rounding and missing data. 
Table 14 (continued). Demographic Characteristics of Sample

\begin{tabular}{lll}
\hline Demographic Characteristic & Categories & $\%(\mathrm{n})$ \\
& & $64.78(160)$ \\
\hline States Where Participants Live & Texas & $28.34(70)$ \\
& Louisiana & $1.62(4)$ \\
& Maryland & $1.62(4)$ \\
& Missouri & $1.21(3)$ \\
& California & $1.21(3)$ \\
& Georgia & $0.81(2)$ \\
& Tennessee & $0.40(1)$ \\
& Florida & $1.62(4)$ \\
& Not Reported & \\
& & $6.80(17)$ \\
Weight Check Frequency & Daily & $14.10(35)$ \\
& Once a Week & $10.40(26)$ \\
& Twice a Month & $36.10(90)$ \\
& Once a Month & $26.90(67)$ \\
& Once Every 6 Months & $4(10)$ \\
& Once a Year & $1.60(4)$ \\
& Never & \\
& & $0.40(1)$ \\
& Very Underweight & $8.40(21)$ \\
Perception of Weight & Somewhat Underweight & $43.40(108)$ \\
& Just About Right & $7.60(96)$ \\
& Somewhat Overweight & $38.60(18)$ \\
& Very Overweight & $2(5)$ \\
\hline
\end{tabular}

Note. Because of missing data, total participants of some demographics do not equal 251. Percentages may not add up to $100 \%$ due to rounding and missing data. 
Table 14 (continued). Demographic Characteristics of Sample

\begin{tabular}{lll}
\hline Demographic Characteristic & $\%$ & $\mathrm{n}$ \\
\hline Recruitment Sites & & \\
Colleges/Universities & & \\
$\quad$ Xavier University & 39.05 & 66 \\
University of Texas at Austin & 28.40 & 48 \\
Huston-Tillotson University & 5.33 & 9 \\
Texas Christian University & 1.78 & 3 \\
Emory University & 1.18 & 2 \\
Austin Community College & .60 & 1
\end{tabular}

Faith-based Organizations

Missionary Baptist Convention of Texas $5.33 \quad 9$

Mt. Zion Baptist Church $\quad 5.33 \quad 9$

Grace Covenant Christian Center $\quad 2.37 \quad 4$

National Baptist Convention $\quad 1.18 \quad 2$

Kids Across America $\quad .60 \quad 1$

Community Events

Kick and Roll Basketball Fundraiser $\quad 2.96 \quad 5$

Family Reunion $\quad 2.96 \quad 5$

Juneteenth Festival in Round Rock $\quad 2.37 \quad 4$

Beauty/Barbershop

Ann's Private Cuts

.60

1

Note. Because of missing data, total participants of some demographics do not equal 251.

Percentages may not add up to $100 \%$ due to rounding and missing data.

\section{DESCRIPTIVE ANALYSES OF THE STUDY VARIABLES}

Descriptive statistics, including instrument reliability, range, means, medians, and standard deviations of the variables used in this study based on the theoretical framework, are described in Table 15 (Chapter 4). Cronbach's alpha coefficients were used to assess the internal consistency of the instruments used in this study. As seen in Table 15, the reliability estimates ranged from .63 to .93 . All the instruments used in this study, except 
two instruments (Family Support for Heart Healthy Eating Habits Scale and Friend Support for Heart Healthy Eating Habits Scale), had acceptable internal consistency (Fitzpatrick, 1998).

Table 15. Descriptive Statistics of Study Variables $(N=251)$

\begin{tabular}{|c|c|c|c|c|c|c|c|c|}
\hline Instrument/Scale & $\begin{array}{l}\text { \# of } \\
\text { Items }\end{array}$ & $\alpha$ & Variable & $\begin{array}{l}\text { Possible } \\
\text { Score } \\
\text { Range }\end{array}$ & $\begin{array}{l}\text { Score } \\
\text { Range } \\
\text { Sample }\end{array}$ & Mean & Median & $S D$ \\
\hline \multirow[t]{2}{*}{$\begin{array}{l}\text { Block Dietary } \\
\text { Fat Screener }\end{array}$} & 17 & .84 & $\begin{array}{l}\text { Dietary Fat } \\
\text { Intake }\end{array}$ & $0-68$ & $4-56.10$ & 24.75 & 24.44 & 10.65 \\
\hline & & & BMI & NA & $15-41.80$ & 25.47 & 24.97 & 4.92 \\
\hline $\begin{array}{l}\text { Intrinsic } \\
\text { Religiosity } \\
\text { Subscale }\end{array}$ & 3 & .90 & $\begin{array}{l}\text { Religious } \\
\text { Commitment }\end{array}$ & $3-15$ & $3-15$ & 12.20 & 13 & 3.26 \\
\hline $\begin{array}{l}\text { Economic } \\
\text { Adequacy Scale }\end{array}$ & 7 & .91 & $\begin{array}{l}\text { Income } \\
\text { Adequacy }\end{array}$ & $7-28$ & $7-28$ & 18.32 & 19 & 5.21 \\
\hline $\begin{array}{l}\text { Healthy Foods } \\
\text { and Snacks } \\
\text { Barriers } \\
\text { Subscale }\end{array}$ & 15 & .82 & Barriers & $15-60$ & $15-56$ & 34.88 & 34 & 7.37 \\
\hline $\begin{array}{l}\text { Resisting } \\
\text { Relapse } \\
\text { Subscale }\end{array}$ & 17 & .93 & Self-Efficacy & $17-85$ & $17-85$ & 57.26 & 58 & 17.04 \\
\hline $\begin{array}{l}\text { Family Support } \\
\text { for Heart } \\
\text { Healthy Eating } \\
\text { Habits Scale }\end{array}$ & 13 & .69 & $\begin{array}{l}\text { Family Social } \\
\text { Support }\end{array}$ & $13-65$ & $22-59$ & 37.73 & 38 & 7.15 \\
\hline $\begin{array}{l}\text { Friend Support } \\
\text { for Heart } \\
\text { Healthy Eating } \\
\text { Habits Scale }\end{array}$ & 10 & .63 & $\begin{array}{l}\text { Friend Social } \\
\text { Support }\end{array}$ & $10-50$ & $17-48$ & 31.10 & 30 & 5.60 \\
\hline $\begin{array}{l}\text { Perceived } \\
\text { Stress Scale }\end{array}$ & 10 & .87 & $\begin{array}{l}\text { Perceived } \\
\text { Stress }\end{array}$ & $0-40$ & $0-37$ & 19.44 & 20 & 7.07 \\
\hline
\end{tabular}




\section{Behavior Outcome (Dietary Fat Intake)}

As seen in Table 15, the sample's DFI scores ranged from 4 to 56.10, with a mean score of $24.75(S D=10.65)$. Dietary fat intake, measured by the score on the Block Dietary Fat Screener (BDFS), was categorized in the following manner: very low fat intake (scores from 0 to 7), average fat intake (scores from 8 to 14), quite high fat intake (scores from 15 to 22), and very high fat intake (scores $\geq 23$ ) (Block et al., 2000). Using the BDFS score categories (Block et al., 2000), the sample's mean DFI score was categorized as very high fat intake. According to the score description on the Blocker Dietary Fat Screener, scores $\geq 23$ mean that probably $40-50 \%$ of one's calories come from fat. As seen in Table 16, the majority of this sample's DFI scores were categorized as quite high fat intake (21\%) or very high fat intake (58\%). A greater percentage of males were categorized as very high fat intake compared to females. Using SPSS, an independent t-test was conducted to determine whether or not the mean DFI scores for males and females are statistically different. The difference between mean DFI scores, 5.61, was significant $(t[75.553]=3.125, p=.003)$.

Table 16. Dietary Fat Intake Categories $(n=236)$

\begin{tabular}{llll}
\hline Category & Sample & Males $(\mathrm{n}=54)$ & Females $(\mathrm{n}=181)$ \\
\cline { 2 - 4 } & $\%(\mathrm{n})$ & $\%(\mathrm{n})$ & $\%(\mathrm{n})$ \\
\hline Very Low Fat Intake & $3(7)$ & $0(0)$ & $3.9(7)$ \\
Average Fat Intake & $17.4(41)$ & $14.8(8)$ & $18.2(33)$ \\
Quite High Fat Intake & $21.2(50)$ & $16.7(9)$ & $22.1(40)$ \\
Very High Fat Intake & $58.5(138)$ & $68.5(37)$ & $55.8(101)$ \\
& & $29.10(12.03)$ & $23.49(9.90)$ \\
\hline
\end{tabular}

Note. Gender was not reported for one of the participants in the sample 236. Percentages may not add up to $100 \%$ due to rounding and missing data. 
Using the formulas provided by Block et al. (2000), the sample's percent of daily calories from fat, total fat intake, total saturated fat intake, and total dietary cholesterol intake were calculated (Table 17). The percent of daily calories from fat ranged from about $25 \%$ to $53 \%(M=36.38, S D=6.22)$, which is high based on the Dietary Guidelines for Americans. The U.S. Department of Agriculture (2010) recommends for adults, ages 19 years and older, to limit their percent of daily calories from fat to $20 \%$ $35 \%$. The total fat intake for this sample, measured in grams, ranged from 51.90 to $167.34(M=100.58, S D=24.83)$. The total saturated fat intake, also measured in grams, ranged from 9.42 to $58.77(M=28.40, S D=9.71)$. Based on the recommendations by the Mayo Clinic (2013) for a 2,000 calorie-a-day-diet (total fat $=44-78$ grams/day and saturated fat $=16-22$ grams/day), this sample's mean fat and saturated fat intake were high. The total dietary cholesterol, measured in milligrams, ranged from 133.15 to 594.18 $(M=306.71, S D=90.03)$. This sample's mean dietary cholesterol intake is higher than the recommendations provided by the U.S. Department of Agriculture (2010), which is consuming less than 300 milligrams of cholesterol per day. Males had a higher percent of daily calories coming from fat and higher intakes of fat, saturated fat, and dietary cholesterol compared to females. Using SPSS, independent t-tests were conducted to determine whether or not the mean percent of daily calories coming from fat and total fat, saturated fat, and dietary cholesterol intakes for males were different from females. The difference between the mean percent of daily calories coming from fat was not statistically different $(t[233]=.785, p=.435)$. The difference between the mean total fat intake was not statistically different $(t[233]=.317, p=.752)$. Statistically significant 
differences were found for total saturated fat intake $(t[233]=5.166, p=.000)$ and total dietary cholesterol intake $(t[233]=6.866, p=.000)$, see Table 17.

Table 17. Fat Intake $(n=235)$

\begin{tabular}{lllll}
\hline Category & Sample & Sample & Males (n=53) & Females (n =182) \\
\cline { 2 - 5 } & Range & Mean (SD) & Mean (SD) & Mean (SD) \\
\hline $\begin{array}{l}\text { Percent Fat } \\
\text { Intake }\end{array}$ & $24.50 \%-53.46 \%$ & $36.38 \%(6.22)$ & $37.03 \%(7.10)$ & $36.19 \%(5.94)$ \\
$\begin{array}{l}\text { Total Fat } \\
\text { Intake (grams) }\end{array}$ & $51.90-167.34$ & $100.58(24.83)$ & $101.64(28.38)$ & $100.28(23.77)$ \\
$\begin{array}{l}\text { Total Saturated } \\
\text { Fat Intake } \\
\text { (grams) }\end{array}$ & $9.42-58.77$ & $28.40(9.71)$ & $34.68(10.41)$ & $26.57(8.71)$ \\
$\begin{array}{l}\text { Total Dietary } \\
\text { Cholesterol }\end{array}$ & $133.15-594.18$ & $306.71(90.03)$ & $380.64(92.24)$ & $285.18(77.26)$ \\
$\begin{array}{l}\text { Intake } \\
\text { (milligrams) }\end{array}$ & & & & \\
\hline
\end{tabular}

\section{Individual Characteristics}

Body Mass Index. As seen in Table 15, the sample's BMI ranged from 15 to 41.80 , with a mean of $25.47(S D=4.93)$. Males and females had similar BMI means (Table 18), 25.42 and 25.43, respectively. According to CDC data from 2007-2010, the average BMI in the U.S. for adult males was 28.6 and 28.7 for females (McCoy, 2013). The mean BMI for both genders in this study is below the national average. According to the BMI categories of the CDC (2015b) described in Table 19, of the 240 participants who reported their height and weight to calculate their BMI, about $50 \%$ of the sample was overweight or obese. A greater percentage of males were overweight or obese $(56.8 \%)$ compared to females $(47.4 \%)$. The percent of obese Black emerging adults in this sample $(18.3 \%)$ is less compared to the general adult population in the U.S. $(34.9 \%)$ 
(CDC, 2014c), Black adults in the U.S. (47.8\%) (Wang \& Beydoun, 2007), and Black adults in the South (39\%) (CDC, 2014b). In a report by Trust for America's Health (2015), obesity rates among emerging adults in all 50 states were less than $28 \%$, which is consistent with this sample (18.3\%). In 2009-2010 the prevalence of obesity was the same for both males and females (36\%) (Weight-control Information Network, 2014). In this sample the prevalence of obesity was slightly higher among females (18.1\%) compared to males $17.6 \%$. The frequency of checking one's weight may affect the accuracy of the self-reported data, such as height and weight. As described in Table 14, the frequency of participants checking their weight ranged from daily to never: daily $(6.8 \%)$, once a week $(14.1 \%)$, twice a month $(10.4 \%)$, once a month $(36.1 \%)$, once every six months (26.9\%), once a year (4\%), and never (1.6\%).

Table 18. Body Mass Index for Males and Females $(\mathrm{n}=243)$

Range Mean SD

\begin{tabular}{llcc}
\hline Males $(\mathrm{n}=52)$ & $15-35.87$ & 25.42 & 4.85 \\
Females $(\mathrm{n}=191)$ & $16.83-41.80$ & 25.43 & 4.91 \\
\hline
\end{tabular}

Table 19. Body Mass Index Classification $(n=240)$

\begin{tabular}{cllll}
\hline & $\begin{array}{l}\text { BMI cut-off } \\
\text { points }\end{array}$ & Sample & Males $(\mathrm{n}=51)$ & Females $(\mathrm{n}=188)$ \\
\cline { 3 - 5 } & $\%(\mathrm{n})$ & $\%(\mathrm{n})$ & $\%(\mathrm{n})$ \\
\hline BMI Category & & & & \\
Underweight & $<18.5$ & $3.3(8)$ & $3.9(2)$ & $3.2(6)$ \\
Normal & $18.5-24.9$ & $47.1(113)$ & $39.2(20)$ & $49.5(93)$ \\
Overweight & $25-29.9$ & $31.3(75)$ & $39.2(20)$ & $29.3(55)$ \\
Obese & $\geq 30$ & $18.3(44)$ & $17.6(9)$ & $18.1(34)$ \\
\hline
\end{tabular}

Note. Since gender was not reported for one of the participants in the sample of 240, the total for males and females reported above is 239 . Percentages may not add up to $100 \%$ due to rounding and missing data. 
Religious Commitment. As seen in Table 15, the sample's scores on the Intrinsic Religiosity Subscale ranged from 3 to 15 , with a mean of $12.20(S D=3.26)$. The distribution of scores for this sample suggests that participants have high internal commitment towards their religious beliefs. Participants were asked how often they attended church or other religious meetings during the year. Half of the sample attends church/religious meetings either once a week (27\%) or more than once a week $(23.6 \%)$ (Table 20). This sample has high organizational religiosity, which refers to participation in church activities (Koenig et al., 2001).

Table 20. Church Attendance $(n=237)$

Frequency of Attendance $\%$ n

\begin{tabular}{lll}
\hline Never & 6.8 & 16 \\
Once a year or less & 8.9 & 21 \\
Few times a year & 13.9 & 33 \\
Few times a month & 19.8 & 47 \\
Once a week & 27 & 64 \\
More than once a week & 23.6 & 56 \\
\hline
\end{tabular}

Income Adequacy. As seen in Table 15, the sample's scores on the Economic Adequacy Scale ranged from 7 to 28 , with a mean of $18.32(S D=5.21)$. Higher income adequacy scores indicate greater perceptions of having adequate income to meet one's daily needs. The distribution of scores for this sample suggests that on average participants perceived themselves as having adequate income to meet their needs.

\section{Behavior-specific Cognitions and Affect}

Perceived Barrier for Healthy Eating. The sample's scores on the Healthy Foods and Snacks-Barriers Subscale (Table 15) ranged from 15 to 56, with a mean of 34.88 ( $S D$ 
$=7.37)$. Higher scores on this scale indicate more barriers to engaging in healthy eating behaviors. The perceived barrier mean of this sample is below the midpoint of 37.5. The distribution of scores for this sample suggests that on average participants perceived themselves to have less barriers for healthy eating.

Perceived Self-efficacy Related to Managing DFI. The sample's scores on the Resisting Relapse Subscale (Table 15) ranged from 17 to 85, with a mean of 57.26 ( $S D=$ 17.04). Higher scores indicate greater self-efficacy for managing DFI. The distribution of scores for this sample suggests that on average participants are confident in managing their DFI.

Perceived Family Support. The sample's scores on the Family Support for Heart Healthy Eating Habits Scale (Table 15) ranged from 22 to 59, with a mean of 37.73 (SD $=7.15)$. Higher scores on this scale indicate greater family support for healthy eating. The perceived family support mean of this sample is below the midpoint of 39 . The distribution of scores for this sample suggests that on average participants perceived themselves to have less family support for healthy eating.

Perceived Friend Support. The sample's scores on the Friend Support for Heart Healthy Eating Habits Scale (Table 15) ranged from 17 to 48, with a mean of 31.10 (SD $=5.60)$. Higher scores on this scale indicate greater friend support for healthy eating. The perceived friend support mean of this sample is above the midpoint of 30 . The distribution of scores for this sample suggests that on average participants perceived themselves to have friend support for healthy eating. 
Perceived Stress. The sample's scores on the Perceived Stress Scale (Table 15) ranged from 0 to 37, with a mean of $19.44(S D=7.07)$. Higher scores indicate greater stress. The perceived stress mean of this sample is below the midpoint of 20 . The distribution of scores for this sample suggests that on average participants perceived themselves to have low stress.

\section{Differences between Paper and Online Survey}

Using SPSS, independent t-tests were conducted to determine whether or not the mean scores on the instruments used in this study for participants who took the online and paper version of the survey were statistically different. The results are reported in Table 21. The difference between the mean religious commitment scores, -1.38 , was statistically significant $(t[234]=-3.21, \mathrm{p}=.002)$. Since gender is a categorical variable, a chi-square test was conducted in SPSS to determine differences in gender (male and female) on the paper and online surveys, reported in Table 22. There was a significant relationship between the type of survey and whether or not participants were male or female: $\chi^{2}(1)=16.17, p=.000$. Female participants tended to take the online survey more compared to males. There were no other statistically significant differences found between the mean scores on paper and online surveys. Because there were no statistically significant differences in the outcome variable (DFI), there was no need to control for survey type (paper and online). 
Table 21. Online and Paper Survey Comparisons for Continuous Variables

\begin{tabular}{llllll}
\hline Variable & Paper (n) & Paper M(SD) & Online (n) & Online M(SD) & $t$ \\
\hline DFI & 41 & $28.36(10.87)$ & 196 & $24(10.47)$ & -2.408 \\
BMI & 36 & $25.55(4.65)$ & 208 & $25.45(4.98)$ & -.109 \\
Religious Commitment & 40 & $13.35(2.25)$ & 196 & $11.97(3.38)$ & $-3.213^{* *}$ \\
Income & 40 & $19.71(5.88)$ & 190 & $18.03(5.03)$ & -1.865 \\
Perceived Barriers & 41 & $35.91(7.95)$ & 182 & $34.63(7.24)$ & -1.004 \\
Perceived Self-efficacy & 41 & $58.30(16.52)$ & 177 & $57.02(17.20)$ & -.433 \\
Perceived Family Social Support & 41 & $37.13(6.47)$ & 170 & $37.87(7.32)$ & .597 \\
Perceived Friend Social Support & 41 & $32.32(5.56)$ & 166 & $30.80(5.59)$ & -1.569 \\
Perceived Stress & 40 & $19.07(6.18)$ & 167 & $19.53(7.28)$ & .371 \\
\hline
\end{tabular}

Note. DFI = Dietary Fat Intake; BMI = Body Mass Index.

$* * p<.01$

Table 22. Online and Paper Survey Comparisons for Categorical Variables

\begin{tabular}{llllll}
\hline Variable & Paper $(\mathrm{n})$ & Paper $(\%)$ & Online $(\mathrm{n})$ & Online $(\%)$ & Pearson Chi-Square \\
\hline Gender & & & & & $16.72^{* * * *}$ \\
Male & 19 & 34 & 37 & 66 & \\
Female & 22 & 11 & 172 & 89 & \\
\hline$* * * p<.001$ & & & &
\end{tabular}




\section{FINDINGS RELATED TO THE RESEARCH QUESTIONS}

This section presents the findings of the study's research questions. The first research question was answered using bivariate correlations. Univariate multiple regression analyses were used to explore the second research question. Hierarchical multiple regression was used to explore the third, fourth, and fifth research questions. Since no relationships were found among the independent and dependent variables identified in Research Question 6, the sixth research question was not analyzed.

\section{Research Question 1}

What are the relationships among the individual characteristics (gender, BMI, income adequacy, and religious commitment), behavior-specific cognitions and affect (perceived barriers for healthy eating, perceived self-efficacy related to dietary fat intake, perceived family social support, perceived friend social support, and perceived generalized stress), and dietary fat intake among Black emerging adults?

The results of the relationships among independent and dependent variables are

presented in Table 23. There were several statistically significant small correlations found among the independent variables. Statistically significant small negative correlations were identified for: a) gender and income with females having lower income adequacy scores compared to males $\left(r_{p b}=-.13, p<.05\right)$; b) perceived stress and income $(r=-.16, p$ $<.05)$; c) perceived stress and perceived self-efficacy $(r=-.15, p<.05)$; and d) perceived stress and perceived family support $(r=-.16, p<.05)$. A statistically significant moderate negative correlation was identified between perceived barriers and perceived self-efficacy $(r=-.40, p<.001)$. Statistically significant small positive correlations were identified 171 
between perceived family support and perceived friend support $(r=.16, p<.05)$ and perceived stress and gender with females having higher perceived stress scores compared to males $\left(r_{p b}=.17, p<.05\right)$.

Among the independent variables and DFI, there were three statistically significant findings. A small negative correlation was identified for gender and DFI $\left(r_{p b}=\right.$ $-.22, p<.001)$, with males having higher DFI scores compared to females. A moderate positive correlation was found between perceived barriers and DFI $(r=.32, p<.001)$. This finding suggests that participants with more barriers to healthy eating tended to consume more dietary fat. A moderate negative correlation was found between perceived self-efficacy and DFI $(r=-.33, p<.001)$. Participants with more self-efficacy tended to consume less dietary fat.

When multiple imputations were conducted to account for missing data, the statistically significant correlations identified above continued to be significant. All of the correlation values for each variable were in the same direction as the values reported in Table 23. Therefore, the results are not influenced by the missing data. 
Table 23. Correlation Matrix for Study Variables $(N=251)$

\begin{tabular}{|c|c|c|c|c|c|c|c|c|c|c|}
\hline Variable & 1 & 2 & 3 & 4 & 5 & 6 & 7 & 8 & 9 & 10 \\
\hline 1. Gender & $1.00^{\mathrm{a}}$ & & & & & & & & & \\
\hline 2. BMI & $.01^{\mathrm{a}}$ & 1.00 & & & & & & & & \\
\hline $\begin{array}{l}\text { 3. Religious } \\
\text { Commitment }\end{array}$ & $.13^{\mathrm{a}}$ & .06 & 1.00 & & & & & & & \\
\hline 4. Income & $-.13^{\mathrm{a}^{*}}$ & -.01 & -.01 & 1.00 & & & & & & \\
\hline 5. Perceived Barriers & $-.03^{\mathrm{a}}$ & .12 & -.09 & -.06 & 1.00 & & & & & \\
\hline 6. Self- Efficacy & $-.03^{\mathrm{a}}$ & .08 & .02 & .06 & $-.40^{* * * *}$ & 1.00 & & & & \\
\hline 7. Family Social Support & $.04^{\mathrm{a}}$ & .09 & .11 & .07 & -.08 & .13 & 1.00 & & & \\
\hline 8. Friend Social Support & $.00^{\mathrm{a}}$ & .08 & .11 & -.05 & -.06 & .09 & $.16^{*}$ & 1.00 & & \\
\hline 9. Perceived Stress & $.17^{\mathrm{a}^{*}}$ & .09 & -.08 & $-.16^{*}$ & .09 & $-.15^{*}$ & $-.16^{*}$ & .04 & 1.00 & \\
\hline 10. DFI & $-.22^{\mathrm{a}^{* * *}}$ & -.06 & -.02 & -.03 & $.32^{* * * *}$ & $-.33^{* * *}$ & -.11 & -.05 & .04 & 1.00 \\
\hline
\end{tabular}

Note. BMI = Body Mass Index DFI = Dietary Fat Intake. Pairwise deletion used for bivariate correlation.

$* p<.05 \quad p<.001 * * *$

a. Point biserial correlation 


\section{Research Question 2}

What are the significant predictors for dietary fat intake among the independent variables of individual characteristics (gender, BMI, income adequacy, and religious commitment) and behavior-specific cognitions and affect (perceived barriers for healthy eating, perceived self-efficacy related to dietary fat intake, perceived family social support, perceived friend social support, and perceived generalized stress) for Black emerging adults?

Based on the results of Research Question 1, variables with significant correlations with DFI were examined, which were the following: gender $\left(r_{p b}=-.22, p<\right.$ $.001)$, perceived barriers $(r=.32, p<.001)$, and perceived self-efficacy $(r=-.33, p<$ .001). Simultaneous multiple regression analysis was conducted to examine whether or not these three variables were significant predictors for DFI. Gender, perceived barriers, and perceived self-efficacy were entered into a model for Research Question 2. Due to the missing data, 217 cases were included for the regression analysis (pairwise deletion). Dietary fat intake scores were regressed on gender, perceived barriers, and perceived selfefficacy.

Preliminary analyses were conducted to ensure that no violation of the assumptions of normality, linearity, multicollinearity, homoscedasticity, and independence of errors occurred. With the outliers identified in 
Table 10 and participant \#524 included in the analysis, the overall multiple regression was statistically significant $\left(\mathrm{R}^{2}=.203, \mathrm{R}^{2}\right.$ adj $=.193, F[3,228]=19.424, p<$ .001 ) and explained $20.1 \%$ of the variance in dietary fat intake. When the outliers and participant \#524 were excluded from the analysis, the overall multiple regression remained statistically significant $\left(\mathrm{R}^{2}=.201, \mathrm{R}_{\text {adj }}^{2}=.190, F[3,213]=17.913, p<.001\right)$ and explained $20.1 \%$ of the variance in dietary fat intake.

Each of the three independent variables (gender, perceived barriers, and perceived self-efficacy) also had a statistically significant effect on DFI, as described in Table 24. The unstandardized regression coefficient $(b)$ for gender was $-5.671(t[216]=-3.629, p=$ .000 ), meaning that female participants' DFI scores were 5 points lower compared to males. The unstandardized regression coefficient $(b)$ for perceived barriers was .310 $(t[216]=3.212, p=.002)$. For each point increase in barrier scores then participants' DFI scores increased by .3 points. The unstandardized regression coefficient $(b)$ for perceived self-efficacy was $-.158(t[216]=-3.783, p=.000)$. For each point increase in selfefficacy scores then participants' DFI scores decreased by .1 points. When the outliers and participant \#524 were included in the analysis, the gender, perceived barriers, and perceived self-efficacy continued to have a statistically significant effect on DFI, having similar coefficient values as reported in Table 24 . 
For Research Question 2, when DFI was regressed on gender, perceived barriers, and perceived self-efficacy using multiple imputations, the overall multiple regression was statistically significant (average $\mathrm{R}^{2}=.19$, average $\mathrm{R}^{2}$ adj $=.18, p<.001$ ). The resulting set of unstandardized $(b)$ and standardized $(\beta)$ regression coefficients were similar to the values reported in Table 24 , in the same direction, and statistically significant. Therefore, the results for Research Question 2 were not influenced by the missing data. The pooled $\mathrm{B}, t$, and $p$ for multiple imputation results are listed in Table 25 . 
Table 24. Regression Weights of Gender, Perceived Barriers, and Perceived Self-Efficacy $(n=216)$

\begin{tabular}{|c|c|c|c|c|c|}
\hline \multirow[b]{2}{*}{ Variables } & \multicolumn{2}{|c|}{ Unstandardized Coefficients } & \multirow{2}{*}{$\begin{array}{l}\text { Standardized } \\
\text { Coefficients } \\
\text { Beta }\end{array}$} & \multirow[b]{2}{*}{$t$} & \multirow[b]{2}{*}{$p$} \\
\hline & B & Std. Error & & & \\
\hline Intercept & 27.382 & 5.085 & & 5.385 & .000 \\
\hline Gender & -5.671 & 1.563 & -.222 & -3.629 & .000 \\
\hline Perceived Barriers & .310 & .096 & .214 & 3.212 & .002 \\
\hline Perceived Self-efficacy & -.158 & .042 & -.252 & -3.783 & .000 \\
\hline
\end{tabular}

Table 25. Pooled B, $t$ and $p$ values for Multiple Imputations

\begin{tabular}{lrrrl}
\hline Variables & B & Std. Error & $t$ & $p$ \\
\hline (Constant) Intercept & 20.266 & 5.238 & 3.869 & .000 \\
Gender & -5.884 & 1.611 & -3.652 & .000 \\
Perceived Barriers & .432 & .097 & 4.430 & .000 \\
Perceived Self-efficacy & -.099 & .041 & -2.409 & .016 \\
\hline
\end{tabular}




\section{Research Question 3}

After controlling for individual characteristics (gender, BMI, income adequacy, and religious commitment), what are the significant predictors for dietary fat intake among Black emerging adults?

In order to take into account the variation explained by gender, BMI, income adequacy, and religious commitment, hierarchical regression was used to answer the above question. Individual characteristic variables were selected based on the theoretical framework and entered into the first block of the model: gender, BMI, income adequacy, and religious commitment. Perceived barriers and perceived self-efficacy were entered into the second block of the model. Then dietary fat intake was regressed on these independent variables. The purpose of this research question was to determine if perceived barriers and perceived self-efficacy have an effect on DFI, even after statistically controlling for the effects of individual characteristic variables (gender, BMI, income adequacy, and religious commitment). The results of the analysis are shown in Table 26. Compared to the results in Research Question 2 in which gender, perceived barriers, and perceived self-efficacy explained $20.1 \%$ of the variance in DFI, the predictor variables entered in the first block of the model for Research Question 3 (gender, BMI, income adequacy, and religious commitment) explained $5.7 \%$ of the variance in DFI and was statistically significant $\left(\mathrm{R}^{2}=.057, \mathrm{R}^{2}\right.$ adj $=.039, F[4,208]=$ $3.146, p=.015)$. The predictor variables entered in the second block of the model for

Research Question 3 (perceived barriers and perceived self-efficacy) accounted for an 
additional $15.2 \%$ of the variance in DFI and was also statistically significant $\left(\mathrm{R}^{2}=.209\right.$, $\mathrm{R}^{2}$ adj $=.186, F[6,206]=9.072, p=.000$ ). The first step of the hierarchical regression (first block of the model), in which gender, BMI, income adequacy, and religious commitment were entered, resulted in the following: $\left.\Delta \mathrm{R}^{2}=.057, F[4,208]=3.146, p=.015\right)$. Of greater interest are the results of the second step of the hierarchical regression. In this step, perceived barriers and perceived self-efficacy were entered in Block 2. These variables explained a statistically significant increase in the variance of DFI scores $\left(\Delta R^{2}\right.$ $=.152, F[2,206]=19.788, p=.000)$.

In the first model only gender had a statistically significant effect on DFI, as described in Table 26. The unstandardized regression coefficient $(b)$ for gender was $5.894(t[212]=-3.377, p=.001)$, meaning that female participants' DFI scores were almost 6 points lower compared to males. In the second model, gender $(b=-5.894$, $t[212]=-3.665, p=.000)$, perceived barriers $(b=.331, t[212]=3.331, p=.001)$, and perceived self-efficacy $(b=-.150, t[212]=-3.513, p=.001)$ had statistically significant effects on DFI. Female participant DFI scores continued to be almost 6 points lower compared to males in the second model. For each point increase in barrier scores then participants' DFI scores increased by .3 points. For each point increase in self-efficacy scores then participants' DFI scores decreased by about .2 points. When the outliers and participant \#524 were included in the analysis, the findings were similar to the values reported in Table 26, with gender, perceived barriers, and perceived self-efficacy continuing to have a statistically significant effect on DFI and having similar coefficient values in the same direction and statistically significant. 
Multiple imputations were conducted to assess the influence of missing data for Research Question 3. The predictor variables entered in the first block of the model (gender, BMI, income adequacy, and religious commitment) remained statistically significant (average $\mathrm{R}^{2}=.0542, p<.05$ ). The predictor variables entered in the second block of the model (perceived barriers and perceived self-efficacy) also remained statistically significant (average $\mathrm{R}^{2}=.197, p<.001$ ). The resulting set of unstandardized (b) regression coefficients were similar to the values reported in Table 26, in the same direction, and statistically significant. The results for Research Question 3 were not influenced by the missing data. The pooled $\mathrm{B}, t$, and $p$ for multiple imputation results are listed in Table 27. 
Table 26. Hierarchical Regression to Predict Dietary Fat Intake among Black Emerging Adults $(\mathrm{n}=212)$

\begin{tabular}{lccccccccc}
\hline \multirow{2}{*}{ Variables } & \multicolumn{4}{c}{ Model 1 } & \multicolumn{5}{c}{ Model 2 } \\
\cline { 2 - 10 }$y$ & $\mathrm{~B}$ & Beta & \multicolumn{1}{c}{$t$} & $p$ & $\mathrm{~B}$ & Beta & $t$ & $p$ \\
\hline Intercept & 34.768 & & 6.463 & .000 & 30.246 & & 4.452 & .000 \\
Gender & -5.894 & -.231 & -3.377 & .001 & -5.894 & -.231 & -3.665 & .000 \\
BMI & -.139 & -.064 & -.955 & .341 & -.161 & -.074 & -1.176 & .241 \\
Income Adequacy & -.130 & -.064 & -.939 & .349 & -.073 & -.036 & -.571 & .568 \\
Religious Commitment & .041 & .012 & .182 & .855 & .126 & .038 & .611 & .542 \\
Perceived Barriers & & & & & .331 & .229 & 3.331 & .001 \\
Perceived Self-efficacy & & & & & -.150 & -.240 & -3.513 & .001 \\
\hline
\end{tabular}

Note. BMI = Body Mass Index.

Table 27. Hierarchical Regression: Pooled B, $t$ and $p$ values for Multiple Imputations

\begin{tabular}{lrrcrcc}
\hline & \multicolumn{3}{c}{ Model 1 } & \multicolumn{3}{c}{ Model 2 } \\
\hline Variables & \multicolumn{1}{c}{$\mathrm{B}$} & \multicolumn{1}{c}{$t$} & $p$ & \multicolumn{1}{c}{$\mathrm{B}$} & \multicolumn{1}{c}{$t$} & $p$ \\
Intercept & 29.767 & 5.708 & .000 & 19.505 & 2.760 & .007 \\
Gender & -6.344 & -3.565 & .000 & -6.215 & -3.791 & .000 \\
BMI & .027 & .193 & .847 & -.058 & -.429 & .668 \\
Income Adequacy & -.132 & -.947 & .344 & -.076 & -.584 & .559 \\
Religious Commitment & .182 & .769 & .443 & .254 & 1.117 & .267 \\
Perceived Barriers & & & & .446 & 4.564 & .000 \\
Perceived Self-efficacy & & & & -.094 & -2.280 & .023 \\
\hline
\end{tabular}

Note. BMI = Body Mass Index. 


\section{Research Question 4}

Do perceived family social support and perceived friend social support moderate the effects of individual characteristics (gender, BMI, income adequacy, and religious commitment) on dietary fat intake?

As previously outlined in Chapter 3, standard procedures for moderation testing, such as centering of variables, were used. Hierarchical multiple regression analysis was used to test for a moderation effect of perceived family support. As described in Table 28 , both gender and the interaction between gender and perceived family support accounted for about $5 \%$ of the variance in DFI. Body mass index, income adequacy, and religious commitment all accounted for less than $1 \%$ of the variance in DFI. The negative values for Adjusted $\mathrm{R}^{2}$ in Table 28 mean that little variance in DFI is accounted for by those variables, not a good fit. Based on the findings, the interaction of perceived family support with gender, BMI, income adequacy, and religious commitment were not statistically significant. Perceived family support did not moderate the effect of gender, BMI, income adequacy, and religious commitment on DFI.

When the outliers and participant \#524 were included in the analysis, the findings were similar to the values reported in Table 28. The interactions of perceived family support with gender, BMI, income adequacy, and religious commitment were not statistically significant. Perceived family support did not moderate the effect of gender, BMI, income adequacy, and religious commitment on DFI. 
Multiple imputations were conducted to assess the influence of missing data for Research Question 4. The resulting set of unstandardized $(b)$ regression coefficients were similar to the values reported in Table 28. The interactions of perceived family support with gender, BMI, income adequacy, and religious commitment were not statistically significant in the multiple imputation results. Perceived family support did not moderate the effect of gender, BMI, income adequacy, and religious commitment on DFI. The results for Research Question 4 in testing the moderation effects of perceived family support were not influenced by the missing data. The pooled $\mathrm{B}, t$, and $p$ for multiple imputation results are listed in Table 29. 
Table 28. Testing Moderation Effects of Perceived Family Support by Regression

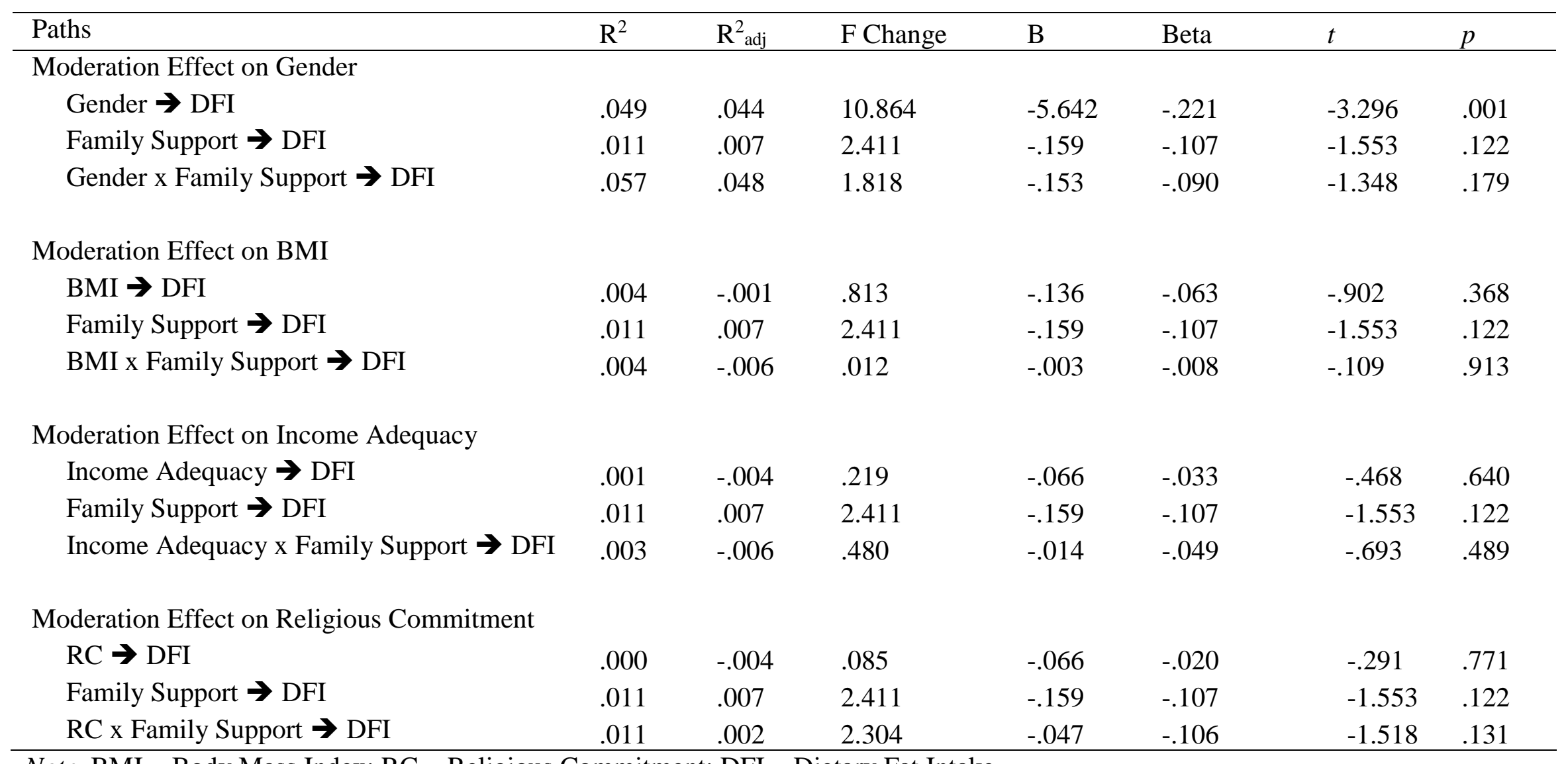

Note . $\mathrm{BMI}=$ Body Mass Index $; \mathrm{RC}=$ Religious Commitment; DFI = Dietary Fat Intake. 
Table 29. Testing Moderation Effects of Perceived Family Support: Average $\mathrm{R}^{2}$, Pooled $\mathrm{B}, t$ and $p$ values for Multiple Imputations

\begin{tabular}{|c|c|c|c|c|c|c|}
\hline Paths & Average $\mathrm{R}^{2}$ & Average $R_{\text {adj }}^{2}$ & Average F Change & $\mathrm{B}$ & $t$ & $p$ \\
\hline \multicolumn{7}{|l|}{ Moderation Effect on Gender } \\
\hline Gender $\rightarrow$ DFI & .0476 & .0432 & 11.3866 & -6.001 & -3.229 & .001 \\
\hline Family Support $\rightarrow$ DFI & .011 & .006 & 2.459 & -.163 & -1.568 & .117 \\
\hline Gender x Family Support $\rightarrow$ DFI & .055 & .0466 & 1.7578 & -.154 & -1.326 & .185 \\
\hline \multicolumn{7}{|l|}{ Moderation Effect on BMI } \\
\hline $\mathrm{BMI} \rightarrow \mathrm{DFI}$ & .0002 & -.0048 & .0292 & .018 & .120 & .904 \\
\hline Family Support $\rightarrow$ DFI & .011 & .006 & 2.459 & -.163 & -1.568 & .117 \\
\hline BMI x Family Support $\rightarrow$ DFI & .0022 & -.0068 & .5178 & -.012 & -.719 & .472 \\
\hline \multicolumn{7}{|l|}{ Moderation Effect on Income Adequacy } \\
\hline Income Adequacy $\rightarrow$ DFI & .001 & -.004 & .150 & -.059 & -.387 & .657 \\
\hline Family Support $\rightarrow$ DFI & .011 & .006 & 2.459 & -.163 & -1.568 & .117 \\
\hline Income Adequacy $*$ Family Support $\rightarrow$ DFI & .003 & -.006 & .439 & -.014 & -.662 & .508 \\
\hline \multicolumn{7}{|l|}{ Moderation Effect on Religious Commitment } \\
\hline $\mathrm{RC} \rightarrow \mathrm{DFI}$ & .001 & -.004 & .174 & .102 & .417 & .677 \\
\hline Family Support $\rightarrow$ DFI & .011 & .006 & 2.459 & -.163 & -1.568 & .117 \\
\hline RC x Family Support $\rightarrow$ DFI & .011 & .002 & 2.303 & -.050 & -1.517 & .129 \\
\hline
\end{tabular}

Note. BMI = Body Mass Index; RC = Religious Commitment; DFI = Dietary Fat Intake. 
Next the second part of Question 4 was examined (test moderation effects of perceived friend support). Using the same steps as for testing the moderation effects of perceived family support, hierarchical multiple regression analysis was also used to test for a moderation effect of perceived friend support. As described in Table 30, both gender and the interaction between gender and perceived friend support accounted for about $5 \%$ of the variance in DFI. Body mass index, income adequacy, and religious commitment all accounted for less than $1 \%$ of the variance in DFI. The negative values for Adjusted $\mathrm{R}^{2}$ in Table 30 mean that little variance in DFI is accounted for by those variables, not a good fit. Based on the findings, the interaction of perceived friend support with gender, BMI, income adequacy, and religious commitment were not statistically significant. Perceived friend support does not moderate the effect of gender, BMI, income adequacy, and religious commitment on DFI.

When the outliers and participant \#524 were included in the analysis, the findings were similar to the values reported in Table 30. The interactions of perceived friend support with gender, BMI, income adequacy, and religious commitment were not statistically significant. Perceived friend support did not moderate the effect of gender, BMI, income adequacy, and religious commitment on DFI.

Multiple imputations were conducted to assess the influence of missing data. The resulting set of unstandardized (b) regression coefficients were similar to the values reported in Table 30. The interactions of perceived friend support with gender, BMI, income adequacy, and religious commitment were not statistically significant in the multiple imputation results. Perceived friend support did not moderate the effect of 
gender, BMI, income adequacy, and religious commitment on DFI. The results for the second part of Research Question 4, testing the moderation effects of perceived friend support, are not influenced by the missing data. The pooled $\mathrm{B}, t$, and $p$ for multiple imputation results were listed in Table 31. 
Table 30. Testing Moderation Effects of Perceived Friend Support by Regression

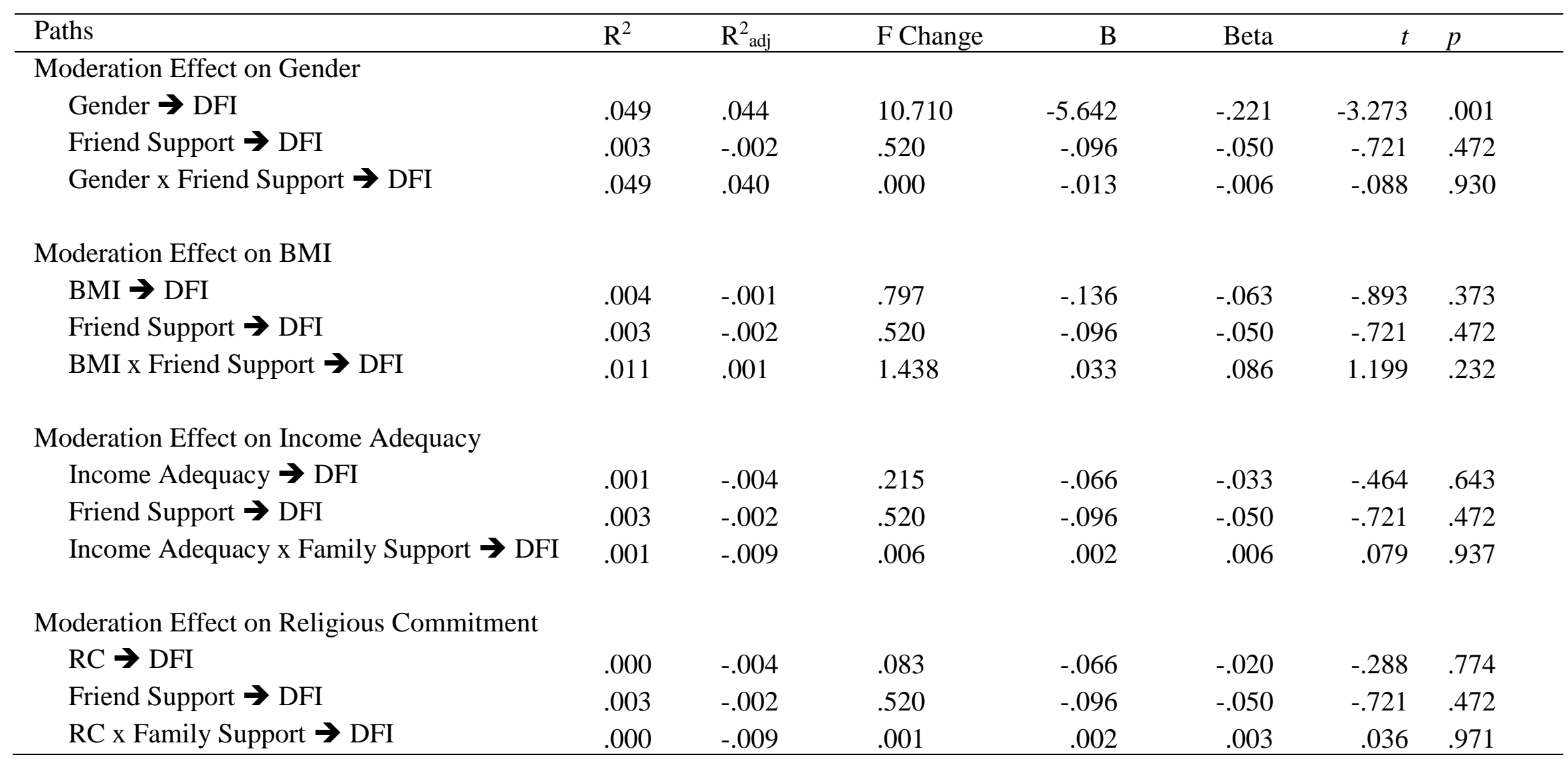

Note $. \mathrm{BMI}=$ Body Mass Index $; \mathrm{RC}=$ Religious Commitment $; \mathrm{DFI}=$ Dietary Fat Intake. 
Table 31. Testing Moderation Effects of Perceived Friend Support: Average $\mathrm{R}^{2}$, Pooled B, $\mathrm{t}$ and $\mathrm{p}$ values for Multiple Imputations

\begin{tabular}{|c|c|c|c|c|c|c|}
\hline Paths & $\mathrm{R}^{2}$ & Average $\mathrm{R}^{2}{ }_{\text {adj }}$ & $\begin{array}{l}\text { Average } \mathrm{F} \\
\text { Change }\end{array}$ & B & $t$ & $p$ \\
\hline \multicolumn{7}{|l|}{ Moderation Effect on Gender } \\
\hline Gender $\rightarrow$ DFI & .048 & .039 & 11.187 & -6.001 & -3.203 & .001 \\
\hline Friend Support $\rightarrow$ DFI & .002 & -.003 & .423 & -.088 & -.650 & .516 \\
\hline Gender x Friend Support $\rightarrow$ DFI & .048 & .039 & .032 & -.026 & -.180 & .857 \\
\hline \multicolumn{7}{|l|}{ Moderation Effect on BMI } \\
\hline $\mathrm{BMI} \rightarrow \mathrm{DFI}$ & .000 & -.005 & .028 & .018 & .119 & .905 \\
\hline Friend Support $\rightarrow$ DFI & .002 & -.003 & .423 & -.088 & -.650 & .516 \\
\hline BMI x Friend Support $\rightarrow$ DFI & .009 & -.001 & 1.840 & .040 & 1.355 & .175 \\
\hline \multicolumn{7}{|l|}{ Moderation Effect on Income Adequacy } \\
\hline Income Adequacy $\rightarrow$ DFI & .001 & -.004 & .147 & -.059 & -.383 & .702 \\
\hline Friend Support $\rightarrow$ DFI & .002 & -.003 & .423 & -.088 & -.650 & .516 \\
\hline Income Adequacy $*$ Family Support $\rightarrow$ DFI & .001 & -.009 & .016 & .003 & .125 & .901 \\
\hline \multicolumn{7}{|l|}{ Moderation Effect on Religious Commitment } \\
\hline $\mathrm{RC} \rightarrow \mathrm{DFI}$ & .001 & -.004 & .170 & .102 & .412 & 680 \\
\hline Friend Support $\rightarrow$ DFI & .002 & -.003 & .423 & -.088 & -.650 & .516 \\
\hline RC x Family Support $\rightarrow$ DFI & .001 & -.008 & .016 & -.007 & -.128 & .898 \\
\hline
\end{tabular}

Note $. \mathrm{BMI}=$ Body Mass Index $; \mathrm{RC}=$ Religious Commitment $; \mathrm{DFI}=$ Dietary Fat Intake. 


\section{Research Question 5}

Does religious commitment moderate the effect of perceived generalized stress on dietary fat intake?

Using the same steps for Question 4, hierarchical multiple regression analysis was also used to test for a moderation effect of religious commitment. As described in Table 32, religious commitment and stress accounted for less than $1 \%$ of the variance in DFI. The interaction between stress and religious commitment accounted for $4 \%$ of the variance in DFI. Based on the findings, the interaction of religious commitment with stress was statistically significant. Religious commitment moderated the effect of stress on DFI. The level of one's religious commitment can influence the relationship between stress and DFI. When the outliers and participant \#524 were included in the analysis, similar results were found as reported in Table 32. The interaction of religious commitment with stress remained statistically significant.

Multiple imputations were conducted to assess the influence of missing data for Research Question 5. The resulting set of unstandardized $(b)$ regression coefficients were similar to the values reported in Table 32, in the same direction, and statistically significant. The interaction of religious commitment with stress remained statistically significant. Religious commitment moderated the effect of stress on DFI. The results for Research Question 5 in testing the moderation effects of religious commitment were not influenced by the missing data. The pooled $\mathrm{B}, t$, and $p$ for multiple imputation results are listed in Table 33. 
In Figure 5 there appears to be a strong positive relationship between perceived stress and DFI among the high religious commitment group (green slope). There appears to be a weak positive relationship between perceived stress and DFI among the low religious commitment group (blue slope). These findings suggest that individuals with both high religious commitment and high perceived stress had high dietary fat intake scores compared to those with low religious commitment scores. 
Table 32. Testing Moderation Effects of Religious Commitment by Regression

\begin{tabular}{|c|c|c|c|c|c|c|c|}
\hline Paths & $\mathrm{R}^{2}$ & $\mathbf{R}_{\text {adj }}^{2}$ & F Change & $\mathrm{B}$ & Beta & $t$ & $p$ \\
\hline \multicolumn{8}{|c|}{ Moderation Effect on Gender } \\
\hline Stress $\rightarrow$ DFI & .013 & .008 & 2.619 & .253 & .113 & 1.618 & .107 \\
\hline $\mathrm{RC} \rightarrow \mathrm{DFI}$ & .000 & -.004 & .095 & -.066 & -.020 & -.309 & .758 \\
\hline Stress $x$ RC $\rightarrow$ DFI & .040 & .031 & 5.823 & .128 & .169 & 2.413 & .017 \\
\hline
\end{tabular}

Note $. \mathrm{RC}=$ Religious Commitment; DFI = Dietary Fat Intake.

Table 33. Testing Moderation Effects of Religious Commitment: Average $\mathrm{R}^{2}$, Pooled $\mathrm{B}, \mathrm{t}$ and $\mathrm{p}$ values for Multiple Imputations

\begin{tabular}{|c|c|c|c|c|c|c|}
\hline Paths & Average $\mathrm{R}^{2}$ & Average $\mathrm{R}^{2}$ adj & Average F Change & $\mathrm{B}$ & $t$ & $p$ \\
\hline \multicolumn{7}{|l|}{ Moderation Effect on } \\
\hline \multicolumn{7}{|l|}{ Gender } \\
\hline Stress $\rightarrow$ DFI & .012 & .007 & 2.638 & .248 & 1.624 & .104 \\
\hline $\mathrm{RC} \rightarrow \mathrm{DFI}$ & .001 & -.003 & .194 & .102 & .441 & .659 \\
\hline Stress $x$ RC $\rightarrow$ DFI & .027 & .018 & 3.298 & .097 & 1.816 & .069 \\
\hline
\end{tabular}

Note $. \mathrm{RC}=$ Religious Commitment; DFI = Dietary Fat Intake. 
Figure 5. Graph of Moderation Effect of Religious Commitment on Relationship between Stress and DFI

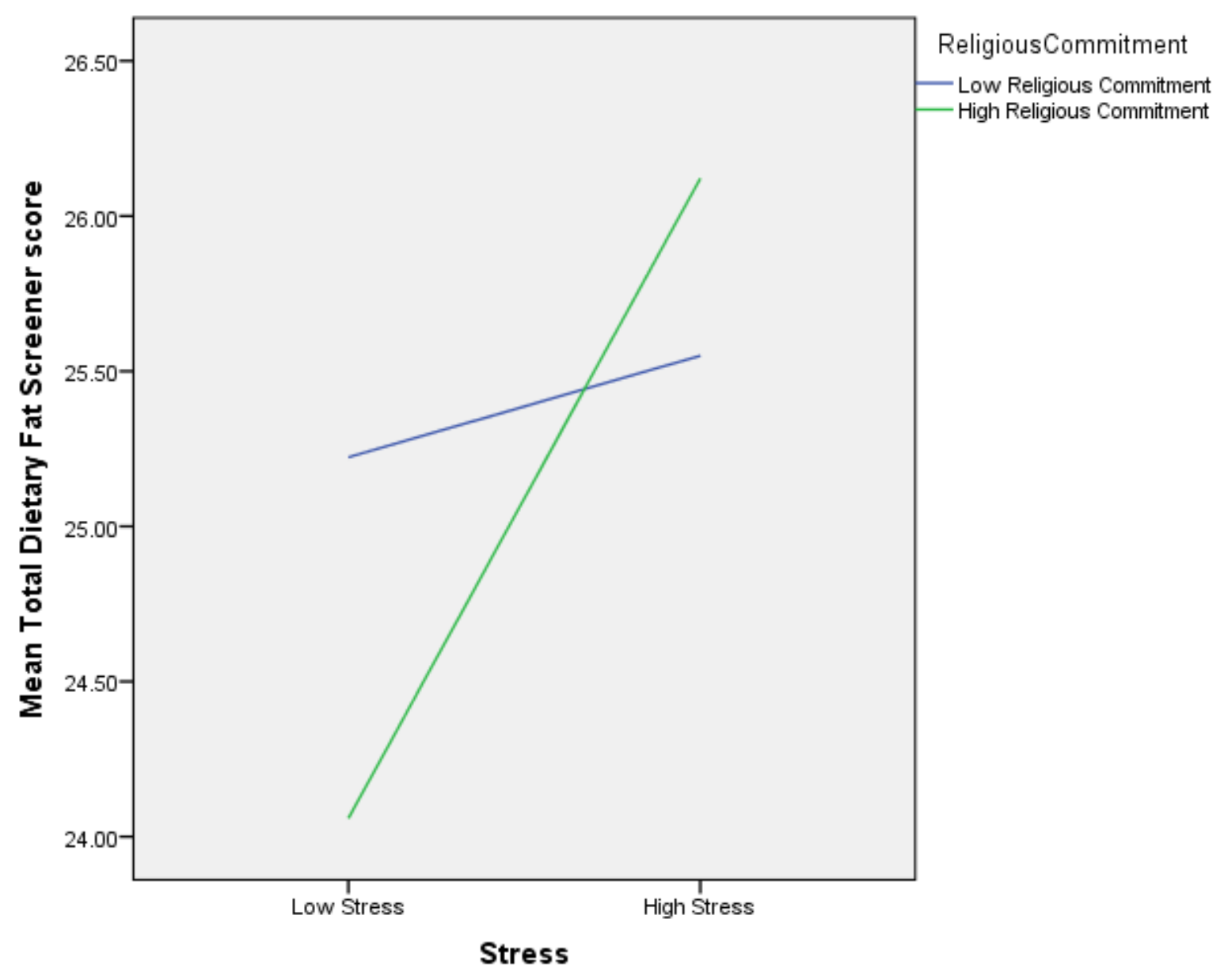




\section{Research Question 6}

Does perceived generalized stress mediate the effect of religious commitment on dietary fat intake?

There was not a direct effect between the independent variable (religious commitment) and the dependent variable (dietary fat intake). Therefore, the mediator effect of perceived stress was not tested. Before a mediator effect can be tested, there has to be a significant direct effect between the independent variable and the dependent variable (Baron \& Kenny, 1986; Bennett, 2000; Warner, 2013).

\section{SUMMARY}

This chapter provided demographic characteristics of the sample of Black emerging adults and descriptive analyses of the variables used in this study. Then findings of the analyses for each research question were described. The identified statistically significant correlations for the outcome variable of DFI were gender $\left(r_{p b}=-\right.$ $.22, p<.001)$ (high fat intake among males), perceived barriers $(r=.32, p<.001)$, and perceived self-efficacy $(r=-.33, p<.001)$. Using simultaneous multiple regressions, it was determined that these three variables were statistically significant predictors for DFI and explained $20.4 \%$ of the variance in dietary fat intake. These three variables had a statistically significant effect on DFI, suggesting that being female, increases one's selfefficacy for managing dietary fat intake, and decreasing barriers for healthy eating were related to better (lower) DFI scores. After controlling for individual characteristics (gender, BMI, income adequacy, and religious commitment), perceived barriers and self- 
efficacy explained a statistically significant increase in the variance of DFI scores; this finding suggests that perceived barriers and perceived self-efficacy may indeed be important for management of DFI among Black emerging adults.

Using hierarchical multiple regression analyses, it was determined that perceived family support and perceived friend support did not moderate the effects of gender, BMI, income adequacy, and religious commitment on DFI. Religious commitment influenced the effects of perceived stress on DFI. Mediating effects were not tested since there was not a direct effect between the independent variable (religious commitment) and the dependent variable (DFI). The presence or absence of outliers and missing data did not affect the results. 


\section{Chapter 5: Results}

This chapter includes a discussion of the study findings, strengths and limitations of the study, implications, and recommendations based on the results. The first section provides a discussion of findings in comparison to previous research and as applicable to the theoretical framework. In the second section, strengths and limitations of the study are described. The implications for nursing, theory, practice, research, and health policy are presented in the third section.

\section{SUMMARY OF THE STUDY}

The purpose of this study was to examine the influence of individual characteristics (gender, BMI, income adequacy, and religious commitment) and behaviorspecific cognitions and affect (perceived barriers for healthy eating, perceived selfefficacy related to managing dietary fat intake, perceived family social support, perceived friend social support, and perceived generalized stress) on the behavior outcome of dietary fat intake (DFI). Potential moderating effects of perceived family social support, perceived friend social support, and religious commitment were also examined. Five research questions, described in Chapter 4, were tested in order to address the purpose of this study.

Using a theoretical framework adapted from the Health Promotion Model, this study used a non-experimental, cross-sectional, descriptive correlational study design. Using Qualtrics, an online survey program, and a paper version of the online survey, data were collected among Black emerging adults at one time period from February 27 to 
September 24, 2014. Both Black male and female participants who are ages 18 to 25 years, live in the U.S., self-identify as Black, speak and read English, and not pregnant were recruited for this study. Participants were recruited through face-to-face visits with organizations (i.e., student organizations and churches), face-to-face visits with local salons and barbershops, social media sites, email, and word of mouth. The initial target sample size was 189 , which was calculated by $\mathrm{G}^{*}$ Power with a significance level of $p=$ .05 , effect size of .09 , and power of .80 . Using $G *$ Power for calculation, the power of this study after data collection (post hoc) was .99, with a final sample size of 251 , significance level of $p=.05$ and effect size of . 23 (Adjusted $\mathrm{R}^{2}=.19$ ).

Data analyses were conducted with missing data and with multiple imputations for missing data. The findings for both were similar and significant when indicated. Since there was no change in the findings, pairwise deletion was used for the data analysis. Using SPSS (version 21), bivariate correlations, univariate multiple regressions, and hierarchical multiple regressions were performed to answer the research questions.

\section{FINDINGS AND DISCUSSIONS}

\section{Sample}

A nonprobability sample of 251 Black emerging adults was used for this study. Most of the sample was recruited through two southern universities: Xavier University (39.05\%) and the University of Texas at Austin (28.40\%), which explains why a majority

of the sample live in Texas (64.78\%) and Louisiana (28.34\%). Of those who reported their gender, there were more females (194) compared to males (56). Sander (2012) 
reported that males are less likely to be actively involved in college campus activities and have less drive for academic success compared to females. Males tend to have a "laidback" approach to life, and males of color are the least likely to engage (Sander, 2012). It may be possible that this lack of engagement found among males on college campuses is present in this study's sample. In regards to online surveys, Smith (2008) reported that females might complete surveys because they want to support others. Jackson and colleagues (2001) reported that females' online activity is described as communicating and exchanging information (e.g., interpersonal), while males' online activity is described as information seeking. Females' support of others' research and interpersonal exchange online suggests they are more likely to access and complete an online survey compared to males (Jackson, Ervin, Gardner, \& Schmitt, 2001; Smith, 2008), which was also found in this dissertation study (see Table 22).

A majority of this sample was single (94\%) and lived with their parents $(32.7 \%)$ or a roommate (44.4\%). Sixty-seven percent of the participants in this sample were enrolled in college, which may explain why they live with parents or roommates. Marriage is usually ranked at the bottom for transition into adulthood among emerging adults (Arnett, 2000). In addition, there has been a decline in marriage among this age group (Pew Research Center, 2013a), which may explain the large percentage of single participants in this study.

Body mass index was calculated using the participant's self-report of height and weight and the following formula: $\mathrm{BMI}=$ (weight in kilograms $/$ height in meters ${ }^{2}$ ) (CDC, 2015b). The sample's mean BMI was $25.47(S D=4.92)$ and ranged from 15 to 
41.80, with the mean for males being 25.42 and for females being 25.43. Based on the CDC BMI categories (CDC, 2015b), the mean BMI for this sample is considered overweight. According to CDC data from 2007-2010, the average BMI in the U.S. for all ages of adult males was 28.6 and 28.7 for females (McCoy, 2013). Specifically among college adults, researchers have reported BMI means for males ranging from 24.7-25.5 and 23.6-23.7 for females (American College Health Association, 2007; Morrell, Lofgren, Burke, \& Reilly, 2012). Mackey (2015) reported a BMI mean of 24.28 (SD = 3.57) among a sample of Black college students. This current study's sample has a higher BMI mean compared to the other studies. About $50 \%$ of the sample in this current study was categorized as overweight or obese: underweight (3.3\%), normal (47.1\%), overweight (31.3\%), and obese (18.3\%). Fifty-six percent of males and $47.4 \%$ of females were classified as overweight or obese, which is higher than the $46.9 \%$ for male and 27.2\% for female college students reported by Morrell et al. (2012). This finding is also slightly higher than the $46 \%$ (40\% overweight and $6 \%$ obese) found among Black college students in a study conducted by Mackey et al. (2015).

Income adequacy was measured by the Economic Adequacy Scale. The 7-item scale had a mean score of $18.32(S D=5.21)$, which ranged from 7 to 28 . This moderatehigh mean score suggests this sample perceived their income as adequate. A majority of the participants in this study live with their parents or a roommate. Such living arrangements may help emerging adults save on living expenses (e.g., food, rent, and utilities). If they live with their parents, they may perceive that their parents' income is adequate to meet their needs. 
Religious commitment was measured by the Intrinsic Religiosity Subscale from the Duke University Religion Index. The 3-item scale had a mean score of $12.20(S D=$ 3.26), which ranged from 3 to 15 . Similar to the findings of other researchers that have examined religious variables and found religion to be important to Blacks (Pew Research Center, 2009; Newport, 2006; Taylor \& Chatters, 2010), this sample had a high internal commitment towards their religious beliefs. Although a few recruitment sites included faith-based organizations, this study's sample was not biased toward religious commitment based on recruitment sites since a majority of participants were recruited from colleges/universities.

Perceived barriers for healthy eating was measured by the Healthy Foods and Snacks Barriers Subscale from the Motivators of and Barriers to Health-Smart Behaviors Inventory. The 15-item scale had a mean score of $34.88(S D=7.37)$, which ranged from 15 to 56. The distribution of scores for this sample suggests that participants perceived themselves to have few barriers for healthy eating. Fila and Smith (2006), Horton (2013a), Jenkins and Horner (2005) have found that younger populations encounter barriers when attempting to eat healthy. Since this sample perceives themselves as having adequate income, they may perceive fewer barriers to eating healthy. It is also possible that healthy eating is not a priority among this sample, which would explain their less perceived barriers.

Perceived self-efficacy related to managing DFI was measured by the Resisting Relapse Subscale from the Self-Efficacy for Eating Behaviors Scale. The 17-item subscale had a mean score of $57.26(S D=17.04)$, which ranged from 17 to 85 . The 
distribution of scores for this sample suggests that participants perceived themselves to be confident in managing their DFI. As described later in this chapter, this finding of high confidence in managing their DFI did not correspond with their DFI consumption. It may be possible that the emerging adults in this study had an inflated perception of their selfefficacy. Other researchers have found that young populations think they are above average and able to complete tasks beyond their capability, which may lead to unrealistic expectations and inflated perceptions of their abilities (Dingfelder, 2011; Huffington Post, 2013; Kremer, 2013). In relation to eating habits, Harring, Montgomery, and Hardin (2010) found that college females, with inflated body weight perceptions, did not practice healthy weight management strategies.

Perceived family social support was measured by the Family Support for Heart Healthy Eating Habits Scale. The 13-item scale had a mean score of $37.73(S D=7.15)$, which ranged from 22 to 59 . The distribution of scores for this sample suggests that participants perceived themselves to have less family support for healthy eating. Many of the participants in this sample reported living with their parents. It is possible that they also eat the meals prepared by their parents while living at home. If the meals are not healthy and participants are attempting to make healthy eating changes, they may not perceive their family as supportive. As described in Chapter 2, individuals, who make healthy food choice changes despite their cultural heritage, may find that their family members are not supportive of dietary changes that break cultural traditions (James, 2004; Peters et al., 2006). 
Perceived friend social support was measured by the Friend Support for Heart Healthy Eating Habits Scale. The 10-item scale had a mean score $31.10(S D=5.60)$, which ranged from 17 to 48 . The distribution of scores for this sample suggests that participants perceived themselves to have friend support for healthy eating. It is possible that this sample perceives themselves to have friend support based on their developmental stage. During this time period, establishing their identity overlaps with developing mature interpersonal relationships (Minnesota State University, 2015). It is also possible that friend support increases and parental support decreases as adolescents increase in age (Helsen, Vollebergh, \& Meeus, 2000). Arnett (2010) reported as emerging adults leave home and no longer have daily experiences with their families, the importance of friendship and activities with friends may rise. Social support from friends may also come from online social networking, which increases their potential sources of support from friends (e.g., twitter, instagram, and online applications such as MyFitnessPal). Young adults are more likely to go online compared to other age groups, with $47 \%$ of them using social networking sites (Lenhart et al., 2010). In addition, Black young adults use the mobile web more than other ethnicities/races (Lenhart et al., 2010), which may help them to connect with friends for social support.

Perceived stress was measured by the Perceived Stress Scale. The 10-item scale had a mean score of $19.44(S D=7.07)$, which ranged from 0 to 37 . The distribution of scores for this sample suggests that participants perceived themselves to have low stress. Since a majority of the participants in this study were enrolled in college, they may consider themselves dependent or partially dependent on their parents. This dependence 
may lessen their perceived stress. Emerging adults do not consider themselves to be "adults" until they accept full responsibility for themselves, make independent decisions, and become financially independent (Arnett, 2004). In addition, the sample in this study had high participation in church activities and religious commitment. Their religious commitment may serve as a protective factor in regards to stress, as previously discussed in Chapter 4.

\section{Descriptive Findings and Research Questions}

Outcome Variable: Behavior Outcome-Dietary Fat Intake. Dietary fat intake was measured by the Block Dietary Fat Screener. The 17-item scale had a total mean score of $24.69(S D=10.66)$, which ranged from 4 to 56.10 . Based on the score categories for the Block Dietary Fat Screener (Block et al., 2000), the total mean score is categorized as very high fat intake, which means that probably $40-50 \%$ of one's calories come from fat. In addition, the calculated means for percent of daily calories from fat ( $M$ $=36.34 \%, S D=6.21)$ and total fat intake $(M=100.41$ grams, $S D=24.81)$ were high. Males had higher mean for percent of daily calories from fat (37.03\%) compared to females $(36.19 \%)$. Similar to other researchers who found that most adults and children have excessive fat intake (Kirkpatrick et al., 2012; Wilson, Adolph, \& Butte, 2009), the DFI scores among the sample in this study indicated consumption of high amounts of dietary fat intake. The mean percent of daily calories from fat in this study is higher than the 33-34\% reported in the U.S. (Coulston et al., 2013). It is also higher than findings for Black males (33.7\%) and females (34.4\%) ages 20 years and older, which were reported by Wright and Wang (2010). Morrell et al. (2012) reported a lower percent of daily 
calories from fat among college students compared to this current study: males at $30.2 \%$ and females at $29.7 \%$. However, this sample's calculated mean percent of daily fat calories from fat is less compared to first-year university students in Spain (females = $45.7 \%$ and males $=45.9 \%$ ) (Irazusta et al., 2007). Based on the recommendations by the Mayo Clinic (2013) for a 2,000 calorie-a-day-diet, one's total fat grams/day should range 44-78, which this sample greatly exceeds.

One explanation for a high fat intake among this sample is that younger populations tend to consume more foods high in fat compared to older populations (Anderson, Winett, Wojcik, \& Williams, 2010; Huang et al., 1994; Spencer, 2002). Since a majority of participants were college students, perhaps they chose high-fat foods due to cost. Based on the findings from a pilot study conducted by Horton (2013b), participants reported lack of money as one of the top three barriers in managing their dietary fat intake: "price of healthy foods can be a little outrageous." Another explanation for the high consumption of fat among Black emerging adults may be due to their traditional diet, also known as "southern food" or "soul food." Compared to Whites, Blacks are five times more likely to eat southern foods (American Heart Association, 2013). Foods such as dark green leafy vegetables, legumes, and fruit are considered healthy "soul food" items; however, food preparation practices such as frying meats in fat and adding butter diminish the healthy aspects of these traditional foods (Bailey, 2006; Braithwaite et al., 2009; Huff \& Kline, 1999; Hurt, 2013). 


\section{Research Questions}

\section{Research Question 1}

What are the relationships among the individual characteristics (gender, BMI, income adequacy, and religious commitment), behavior-specific cognitions and affect (perceived barriers for healthy eating, perceived self-efficacy related to dietary fat intake, perceived family social support, perceived friend social support, and perceived generalized stress), and dietary fat intake among Black emerging adults?

In this study, the below variables had significant small relationships.

- Gender and income $\left(r_{p b}=-.13, p<.05\right)$, with females having lower income adequacy scores compared to males. In general, males earn more money than females (Berman, 2014), which may explain females' lower income adequacy scores in this sample.

- Perceived family support and perceived friend support $(r=.16, p<.05)$. This finding suggests that participants with high perceived family social support tended to have high perceived friend social support. Most of the participants in this study were enrolled in college. Perhaps they were receiving support from their families for school and developing new friendships on campus, which may explain this finding.

- Perceived stress and gender $\left(r_{p b}=.17, p<.05\right)$, with females having higher perceived stress scores compared to males. Perhaps the females in this study had role demands (e.g., caregiver) that increased their perceived level of stress. As 
described above, males tend to earn more money than females. Females in this study may have experienced stress related to low income adequacy.

- Perceived stress and income $(r=-.16, p<.05)$. This finding suggests that participants with less perceived adequate income scores tended to have high perceived stress scores. Davidson (2015) reported that low-income households are more stressed about money compared to high-income households, which may explain this finding.

- Perceived stress and perceived self-efficacy $(r=-.15, p<.05)$. This finding suggests that participants with less perceived self-efficacy tended to have high perceived stress scores. Perhaps feeling incapable of successfully managing one's dietary fat intake actually created stress within participants and/or a sense of loss of control in regards to healthy eating (i.e., dietary fat intake).

- Perceived stress and perceived family social support $(r=-.16, p<.05)$. This finding suggests that participants with less perceived family social support tended to have high perceived stress scores. In general, social support helps individuals cope during stressful situations (Baqutayan, 2011; Mills, Reiss, \& Dombeck, 2008). The sample in this study may have used their family social support to handle stress.

In this study, the below variables had significant moderate relationships.

- Perceived barriers and perceived self-efficacy $(r=-.40, p<.001)$. This finding suggests that participants with high perceived barrier scores tended to have low perceived self-efficacy in managing their dietary fat intake. Perhaps 
participants in this study, who had low self-efficacy, also felt overwhelmed by their barriers and/or unable to navigate through barriers.

The following sections describe the relationships between the independent variables and dietary fat intake.

Gender. In this study there was a statistically significant negative correlation between gender and DFI, which was categorized as male or female. On average, males had higher fat intakes compared to females. Similarly, Harcrow (2010) and Hart et al. (2006) found that males had significantly higher fat intakes scores compared to females. Hart and colleagues' (2006) sample in their study ranged from ages 19-100 years $(N=$ $2,375, M$ age $=55, \mathrm{SD}=16$ ), with $88 \%$ identifying as White and $4 \%$ as Black. Harcrow's (2010) sample consisted of undergraduate college students $(N=914, M$ age $=$ 20.58), with $78.2 \%$ identifying as White and $16.7 \%$ as Black. In addition, the mean percent of daily calories from fat for males $(37.03 \%, S D=7.10)$ was higher than females ( $36.14 \%, S D=5.93$ ). This finding is higher than the 2007-2008 fat consumption reported by Wright and Wang (2010), with the average total percent of daily calories from fat at $33.6 \%$ for males and $33.5 \%$ for females age 20 years and older. In particular, Wright and Wang (2010) reported that among Black males the average total percent of daily calories from fat was $33.7 \%$ and $34.4 \%$ for Black females, which is less than reported for this current study. The findings in this current study are also consistent with studies conducted by Cartwright and colleagues (2003) and Spencer (2002). Cartwright et al. (2003) found teenage females had lower odds of consuming high-fat foods (OR = .56[-49-.64], $p=.00$ ) compared to males. Cartwright and colleagues' (2003) sample 
consisted of 4,320 schoolchildren $(M$ age $=11.83)$, with $61.7 \%$ identifying as White and $18.9 \%$ as Black. Spencer (2002) found females were less likely to eat diets high in saturated fats $\left(\chi^{2}=25.01, d f=3, p=.00\right)$ compared to males. Spencer's (2002) sample ranged from ages $18-26$ years $(N=226, M$ age $=21)$, with $84 \%$ identifying as White.

Gender roles expectations, explained by Wansink (2006), may be an explanation for why males in this current study consumed more fat compared to females. Females may consider overeating as not being feminine (Wansink, 2006), in this case consuming too much dietary fat may be considered not feminine. On the other hand, having a healthy appetite may be perceived as "manly" among the males in this study (Wansink, 2006). A perception of having a healthy appetite, which equates to being "manly", may influence how much dietary fat was consumed among males in this current study.

Body mass index. As previously described in Chapter 2, there are mixed findings in the literature regarding the relationship between BMI and DFI. In this study there was not a statistically significant relationship found between BMI and DFI, which is similar to the findings reported by Vadiveloo et al. (2014) and Omondi et al. (2011). As described in Chapter 2, mixed findings have been reported regarding the relationship between BMI and DFI. Positive relationships have been found in studies conducted with rats and mice (Boozer, Schoenbach, \& Atkinson, 1995; Salmon \& Flatt, 1985; Schemmel, Mickelsen, \& Motawi, 1972). Non-significant relationships among human populations may be due to multiple factors such as age, gender, genetics, metabolism, rate of fat oxidation, and level of physical activity (Blundell \& Macdiarmid, 1997; Bray \& Popkin, 1998; Omondi et al., 2011; National Heart Foundation of Australia, 2003). For example, Howarth et al. (2005) 
found no significant relationship between BMI and fat intake among females, but they found a significant relationship among males $(B=.09, p=.003)$. In contrast, Ledikwe et al. (2003) found a positive relationship among females $(r=0.26, p<.05)$, but not among males. In this current study, bivariate correlations between BMI and DFI, based on gender, were conducted post hoc. Based on gender, there were no significant correlations between BMI and DFI: males $(r=-24, \mathrm{p}=.097)$ or females $(r=.00, \mathrm{p}=.999)$.

The frequency of how often participants in this current study checked their weight may have affected the accuracy of the self-reported data used to calculate BMI. As described in Table 14, participants checked their weight once a month (36.1\%), once every six months $(26.9 \%)$, once a year $(4 \%)$, or never $(1.6 \%)$. When individuals do not weigh themselves frequently, they may not be able to report an accurate weight.

Income adequacy. As described in Chapter 2, mixed findings have been reported in the literature regarding the relationship between income and DFI. In this study there was not a statistically significant relationship found between income and DFI, which is similar to the findings reported by Deshmuk-Taskar et al. (2007), Jen et al. (2007), and Hart et al. (2006). Compared to prior to the Great Recession 2007-2009, more young adults live with their parents (Pew Research Center, 2013a). In this sample, a majority of the participants live with their parents or a roommate. Such living arrangements may help young adults save on living expenses (e.g., food, rent, and utilities), and they may perceive that their income or their parents' income is adequate enough to eat in a healthy manner. Age may be another factor contributing to the non-significant finding between income and DFI. Although Hart et al. (2006) did not find a statistically significant 
relationship between income and DFI, when they adjusted for age they found a significant relationship with fat intake. The age range of the participants in their study was 19 to 100 years (mean age $=55, S D=16$ ), with $88 \%$ identifying as White and $4 \%$ as Black. Age was not adjusted for in this current study since the sample consisted of only emerging adults.

Religious commitment. There are equivocal findings in the literature about the relationship between religious commitment and DFI. In this study there was not a statistically significant relationship found between religious commitment and DFI, which is similar to the findings reported by Tan et al. (2013), Reeves et al. (2012), Harcrow (2010), and Fitzgibbon et al. (2005). This sample was homogenous with respect to religious commitment, and this non-significant finding may be related to the lack of variability in the data (Goodwin \& Leech, 2006). The reduced variability in religious commitment can be influenced by a ceiling effect (Polit \& Beck, 2012). A ceiling effect is "having scores at or near the highest possible value, which can constrain the amount of upward change possible and also tends to reduce variability in a variable" (Polit \& Beck, 2012, p. 721). Therefore, the relationship between religious commitment and DFI may not be detected due to lack of variability. Similarly ceiling effects have been reported by other researchers studying religious variables (Bufford et al., 1991; Finke \& Stark, 1998; Genia, 2001; Kim \& Sobal, 2004).

Perceived barriers for healthy eating. In this study, a statistically significant positive relationship was found between perceived barriers for healthy eating and DFI ( $r$ $=.32, p<.001)$. Although Lloyd and colleagues' (1995) experimental study was 
conducted among United Kingdom participants, ages 18-55 years $(M=35.1)$, they found similar findings. They used discriminant analysis to identify barriers among groups in reducing their fat intake, they found a statistically significant positive relationship for the barrier of taste (factor coefficient $=.57, p<.01$ ). A reduction in the quality of taste in reduced fat foods influenced participants' fat intake (Lloyd et al., 1995). Similarly, in a study conducted with a sample of Black adults ages 18-70 years $(M=43.9)$, Watters and Satia (2009) identified the barrier of need information to prepare healthy foods as a significant factor influencing fat intake among males.

Emerging adulthood is characterized by change and exploration (Arnett, 2000). The Black emerging adults in this current study may find it challenging to balance life, transition into adulthood, and adopt healthy eating behaviors such as manage one's dietary fat intake. Most of the participants in this current study were enrolled in college; and barriers such as lack of time and access to healthy food on campus may have influenced their ability to manage their dietary fat intake, which may explain the positive relationship found between perceived barriers and DFI. Consistent with the findings of a pilot study conducted among Black emerging adults by Horton (2013b), participants reported barriers encountered at school. For example, one participant shared, "The availability of healthy food is very limited in a college environment and the funds allotted are very thin to provide healthier food." Another participant reported, "I have limited time to study after work so I pick up fast food on the way home."

Perceived self-efficacy related to managing dietary fat intake. As described in Chapter 2, there are similar findings in the literature regarding the relationship between 
perceived self-efficacy and DFI. In this study there was a statistically significant negative relationship found between perceived self-efficacy and DFI, which is similar to the findings reported by Anderson-Bill et al. (2011), Povey et al. (2000), and Armitage and Conner (1999).

In this current study, participants with more self-efficacy tended to consume less dietary fat $(r=-.33, p<.001)$. Despite the expected correlation, this sample's high mean score for perceived self-efficacy $(57.26, S D=17.04)$, which suggests they were confident in managing their DFI, seems contradictory to the sample's very high fat and saturated fat intakes. In general, individuals with high self-efficacy consume less fat as reported by Watters and Satia (2009) and Smith and Owen (1992).

This contrasting finding between this study's sample and the literature may be due to the lack of current research on self-efficacy and DFI among emerging adult populations. It is difficult to tease out self-efficacy and DFI data specific for emerging adults since they are usually included in other age groups. For example, Watters and Satia (2009) included emerging adults in their study. However, their findings for emerging adults were grouped with participants who were ages 20-34 years. When emerging adults are included within other age groups, accurate findings may not be discovered specific for ages 18-25 years.

Other possible explanations for this discrepancy between this study and the literature regarding self-efficacy and DFI are the following: emerging adults' behavior choices are colored by their sense of invincibility; and society sends messages to emerging adults that easy and quick fixes can correct the negative consequences of their 
behavior health choices. When emerging adults perceive themselves as invincible (e.g., "it cannot happen to me"), they will not consider how their negative health behaviors, such as consuming high-fat foods, can cause harm to their bodies (e.g., cardiovascular disease). Such invincibility viewpoints can influence emerging adults' understanding of the trajectory of their behavior choices. Other researchers, whose samples included young adult populations, have reported similar invincibility beliefs and health choices regarding nutrition, risky behaviors (e.g., sexual practices and alcohol and tobacco use), and cardiovascular disease (Allison \& Campbell, 2009; Dumbrell \& Mathai, 2008; Giesbrecht, 1999; Frasca, Ventuneac, Balan, \& Carballo-Diéguez, 2012; Huh, Sami, Abramova, Spruijt-Metz, \& Pentz, 2013). Coupled with invincibility, society sends messages about how to avoid negative consequences for unhealthy choices. For example, in a study conducted among emerging adults, Black focus group participants shared that "quick and easy solutions" are available to remedy health problems so they can "do any behavior that they want (e.g., eat unhealthy) and then have the results of the behavior corrected (e.g., surgery)" (Horton, 2013a, p. 27).

Perceived family and friend social support. The literature does not consistently separate social support into family and friends; therefore, discussion of the findings in relation to the literature is examined as one entity in this section. There were mixed findings in the literature about the relationship between social support and DFI. In this study family social support $(r=-.11)$ and friend social support $(r=-.05)$ had a statistically non-significant negative relationship with DFI. Participants with high social support tended to consume less dietary fat intake. This non-significant finding has also 
been reported by Anderson-Bill et al. (2011), Harcrow (2010), Hermstad et al. (2010), Kim and Sobal (2004), and Thrasher et al. (2004). This non-significant finding may be because there are multiple social, cultural, and environmental factors, which encompass social support and may influence DFI (Hermstad et al., 2010; Brug, 2008). The overlap between these multiple factors may affect the relationship between social support and DFI.

Perceived stress. As described in Chapter 2, greater stress is generally associated with high DFI. However, in this study there was not a statistically significant relationship between perceived stress and DFI $(r=.04)$. In Chapter 2, of the five studies focused on stress and dietary fat intake only two addressed younger populations (children and women 16 years and older $[M=24.7])$, with Blacks making up $14 \%-18.9 \%$ of the samples (Cartwright et al., 2003; Fowles et al., 2012). Blacks tend to be underrepresented in research studies. More research is needed to examine the effects of stress on DFI among Black emerging adults.

Religion is important among Black populations (Pew Research Center, 2009; Newport, 2006; Taylor \& Chatters, 2010), as also found in this study's sample of Black emerging adults. Since perceived stress scores were on average low among this sample, perhaps religious commitment served as a coping skill, which may have influenced the low scores. Positive religious coping skills have been associated with positive psychological adjustment to stress (Ano \& Vasconcelles, 2005; Klaassen, McDonald, \& James, 2006), and high religious coping has been reported among Black adults (Chatters, Taylor, Jackson, \& Lincoln, 2008). In a qualitative study conducted by Horton (2013a) 
among emerging adults, participants discussed how they used religion to cope with stress. For example, one participant explained how religion helped her to deal with stress: "I have a peace and a confidence and an ability to let go of things and to not be anxious over things...I know that God's in control as compared to a lot of people in the workplace who are constantly anxious" (Horton, 2013a, p. 25). Further research is needed to examine stress and DFI among Black emerging adults.

\section{Research Question 2}

What are the significant predictors for dietary fat intake among the independent variables of individual characteristics (gender, BMI, income adequacy, and religious commitment) and behavior-specific cognitions and affect (perceived barriers for healthy eating, perceived self-efficacy related to dietary fat intake, perceived family social support, perceived friend social support, and perceived generalized stress) for Black emerging adults?

The significant predictors for DFI were gender (males having higher fat intakes compared to females), perceived barriers for healthy eating, and perceived self-efficacy related to dietary fat intake. Based on the unstandardized regression coefficients in Table 24, gender had the greatest effect on DFI $(B=-5.671, t[216]=-3.629, p=.000)$. Perceived barriers for healthy eating had the next greatest effect on DFI $(B=.310, t[216]$ $=3.212, p=.002)$, followed by perceived self-efficacy related to dietary fat intake $(B=-$ $.158, t[216]=-3.783, p=.000)$.

In general, research in this area has not used gender as a predictor but rather as descriptive variable. However, Grizzle (2009) and Harcrow (2010) studied gender as a 
predictor for DFI. While Harcrow (2010) found gender to be a significant predictor for DFI among a sample of college students $(B=-2.37, t[912]=-3.40, p=.001)$, Grizzle (2009) did not find it to be a significant predictor of fat-related habits in a sample of police officers. Participants in Grizzle's study (2009) ranged from 24 to 63 years old $(N=$ $289, M=41.7$ years, SD $=7.85$ ), with only 19 participants in the youngest age group of 22-29 years (males $=17$ and females $=2$ ).

The characteristics of the younger participants in Grizzle's study were different from Harcrow's sample (2010). The mean age of Harcrow's undergraduate college sample was $20.58(S D=2.89)$, and there were more female participants $(n=638,70 \%)$ compared to males $(n=271,30 \%)$. This current study shared similar sample characteristics to Harcrow's study (mean age $=20.49$ years $[S D=2.01]$ and $77 \%$ females). These similarities in sample may explain why gender was a significant predictor of DFI in both studies. In general, females tend to value the nutrition content of foods, be more knowledgeable about nutrition, and more likely to read food labels compared to males (Baker \& Wardle, 2003; Misra, 2007; Morse \& Driskell, 2009; Nayga, 2000; Turrell, 1997), which may explain the gender differences in this study.

Barriers among younger populations that have been identified in the literature include not having enough time to eat, availability and taste of foods, food cravings, little care about eating healthy, and influence of media (Fila \& Smith, 2006; French et al., 2001; Horton, 2013a; Jenkins, 2007; Jenkins \& Horner, 2005; Neumark-Sztainer et al., 1999). The literature is sparse for perceived barriers to the healthy eating as a predictor for DFI. However, one study conducted by Watters and Satia (2009) examined perceived 
barriers to healthy eating, which they defined as enabling factors (feel you can afford healthy foods, time/trouble to prepare healthy foods, it is easy to order healthy foods at restaurants, and need information to prepare healthy foods). They found that these enabling factors explained $11 \%$ (Adjusted $\mathrm{R}^{2}$ ) of the variance in total fat intake (grams/day). Watters and Satia (2009) did not report effect sizes in their study. Due to this unreported data, it is not possible to compare effect sizes between this current study and their study. As described in Chapter 2, when adults, including emerging adults, encounter barriers to eating healthy, they may not successfully overcome them, which may lead to increased fat intake.

Similar to the findings in this study, other researchers have found self-efficacy to predict fat intake. Participants who had high self-efficacy reported lower fat intake. The researchers reported beta coefficients slightly higher compared to this study (-.160): Anderson-Bill et al. (2011) reported beta total $=-.21, p<.001$; Povey et al. (2000) reported beta $=-.26, \mathrm{p}<.05$; and Armitage and Conner's (1999) reported beta coefficient was positive $(.33, p<.001)$, meaning that high self-efficacy had a positive effect on one's intention to eat low fat foods. The samples from these studies included emerging adults. However, the study conducted by Anderson-Bill et al. (2011) was mostly made of White participants (90\%), with a mean age of 44.4 years. Povey and colleagues' (2000) and Armitage and Conner's (1999) studies were conducted in the United Kingdom, with mean ages of 41 and 23 years, respectively. Armitage and Conner's (1999) sample was made of undergraduate college students. Race/ethnicity was not reported in the studies conducted in the United Kingdom. As described in Chapter 2, individuals who perceive 
themselves capable of managing their DFI are likely to consume less dietary fat, compared to those who do not perceive themselves capable. More research is needed regarding self-efficacy and DFI among emerging adults to address the age-gap in the literature described in Chapter 2.

\section{Research Question 3}

After controlling for individual characteristics (gender, BMI, income adequacy, and religious commitment), what are the significant predictors for dietary fat intake among Black emerging adults?

After controlling for gender, BMI, income adequacy, and religious commitment, perceived barriers and perceived self-efficacy were statistically significant predictors for DFI. Watters and Satia (2009) controlled for age, education, and BMI and found similar results for self-efficacy. Grizzle's study (2009) did not mention whether controls were used to determine significant predictors for DFI. However, education and income were relatively controlled for since there was limited variability in these variables among the sample (Grizzle, 2009). In Grizzle's study (2009) race/ethnicity, barriers to healthy eating, and self-efficacy explained $26.4 \%$ of the variance in fat-related diet habits among police officers, who were predominantly Black. These findings suggest that consideration of perceived barriers and perceived self-efficacy may indeed be important for management of DFI among Black emerging adults.

\section{Research Question 4}


Do perceived family social support and perceived friend social support moderate the effects of individual characteristics (gender, BMI, income adequacy, and religious commitment) on dietary fat intake?

Little research has been conducted to examine the moderating effects of social support on dietary fat intake, particularly among emerging adults. McKinley (2009) and Wickrama et al. (2012) found social support to be a significant moderator in regards to healthy eating, among college students and older Black samples, respectively. In this study neither perceived family social support nor friend social support moderated the effect of gender, BMI, income adequacy, and religious commitment on DFI. Similarly, Burke et al. (2008) did not find a significant interaction of social support for dietary fat intake; but the sample consisted of adults ranging from 40 to 70 years old. It may be possible that a statistically significant interaction between perceived family social support and perceived friend social support with BMI, income adequacy, and religious commitment was not found because the sample in this current study was homogeneous, which "may weaken detection of a moderator effect" (Bennett, 2000, p. 418). More research is needed regarding the potential moderating effects of perceived family social support and perceived friend social support among Black emerging adults.

\section{Research Question 5}

Does religious commitment moderate the effect of perceived generalized stress on dietary fat intake?

In this study religious commitment moderated the effect of stress on DFI. An individual's level of religious commitment can influence the relationship between stress 
and DFI. Although the correlation between religious commitment and perceived stress was not statistically significant in this study, there was a negative relationship $(r=-.08)$. Participants with high religious commitment scores tended to have low perceived stress scores. In general, researchers have found religious variables (e.g., religious commitment) to be significant protective factors for one's health (Allen et al., 2013; Bowen-Reid \& Harrell, 2002; Ellison, \& Henderson, 2011; Hill, Burdette, \& Idler, 2011; Joshi et al., 2009; Kilbourne, Cummings, \& Levine, 2011; Larson \& Larson, 2003; McDougle et al., 2013; Pargament, 1997). However, in this study, participants with high religious commitment and high perceived stress had high dietary fat intake scores compared to those with low religious commitment scores. Just as the causes of obesity are multifactorial, it is possible that the relationship between religious commitment and dietary fat intake is also complex and multifactorial.

Although Schlundt and colleagues (2008) did not examine the moderating effects of their religious variables, they found that variables, such as religious affiliation, should be taken into account when conducting research related to religion and health. In their study, Schlundt et al. (2008) examined relationships between religious affiliation (denomination) and religious involvement (religious denomination, church attendance, rating of religiousness, and rating of strength/comfort received from religion) with health behaviors (physical activity and dietary behaviors) among Black and White participants $(n=3014)$ ages 18 years and older, who participated in the Nashville REACH 2010 project. Black participants in their study, ages 18-24 years, consisted of the following: males $7.1 \%$ and females $6.9 \%$ (Schlundt et al., 2008). Depending on religious affiliation, 
Schlundt et al. (2008) found that BMI and healthy eating behaviors were high among some groups. Healthy eating behaviors were found among three religious denomination groups: mainstream Protestants, Catholics, and other. Evangelical Christians had less healthy eating behaviors (Schlundt et al., 2008). The researchers found a positive statistical significant correlation between religious involvement and high-fat behaviors ( $r$ $=.10, p<.0001)$, with participants with high religious involvement having high-fat behaviors (Schlundt et al., 2008). There were significant relationships between denomination affiliation and health behaviors; but they were no longer significant once they controlled for demographics (Schlundt et al., 2008). Similarly, in a study conducted among 546 adults (ages 17 to 91 years, male mean age $=42$, female mean age $=44$ ), Kim and Sobal (2004) found higher fat intakes among Conservative Protestant females and “other affiliation" females compared to Catholic females, controlling for demographics and social support. Schlundt and colleagues (2008) suggest that denomination affiliation is confounded with cultural similarities (e.g., age, gender, race, and socioeconomic status).

Other factors that may explain the moderating effect of religious commitment on the relationship between stress and dietary fat intake in this study are the following: 1) religious commitment is a source of stress and 2) importance of food within faith-based settings (e.g., church). Researchers have discussed religion's negative effects on health such as stress due to individuals' interpersonal struggles, conflict with clergy, and perceptions of punishment from God (Ellison \& Henderson, 2011; Koenig et al., 2001; Pargament, 1997). If individuals' religious commitment is a source of internal conflict, 
which forces them to have high expectation of themselves, and/or pressures them to eat a certain way, then religious commitment may be perceived as a stressor among Black emerging adults. As discussed in Chapter 2, individuals who feel stressed tend to consume more than their recommended percentage of energy from fat (Cartwright et al., 2003; Contrada \& Baum, 2011; Dallman, 2010; Fowles et al., 2012; McCann, Warnick, \& Knopp, 1990; Ng \& Jeffery, 2003; Wardle, Steptoe, Oliver, \& Lipsey, 2000), overeat (Jeong \& Kim, 2007; Torres \& Nowson, 2007; Walcott-McQuigg, 1995), and make unhealthy food choices (Contrada \& Baum, 2011; Edmonds, 2010; Kandiah, Yake, Jones, \& Meyer, 2006; Oliver \& Wardle,1999; Steptoe, Lipsey, \& Wardle, 1998).

Lastly, food served within faith-based settings may be seen as a way to connect people to others and their faith and/or solidify one's membership in a group (Mintz \& Du Bois, 2002). As previously discussed in Chapter 2, "Food has traditionally been a catalyst for social interaction...for some Blacks, eating is an 'intimate' or a 'spiritual' experience that is shared with others" (Kittler \& Sucher, 2008, p. 220). Even in church settings, food remains a central part of interactions (Dodson \& Gilkes, 1995). It is possible that high-fat foods (e.g., cookies, cakes) are served frequently in faith-based settings. Black emerging adults may feel stressed when they attempt to manage their dietary fat intake in a faithbased setting (e.g., church) that is serving high-fat foods. Perhaps they want to feel a part of their church community by eating high-fat foods served in their religious communities. If they refuse to eat the high-fat foods offered, it may be interpreted as being rude. The stress in trying to manage one's dietary fat intake and remaining committed to fellowship with one's religious community may explain the interaction between religious 
commitment and stress found in this study. More research is needed to explain the effects of religious commitment. Future research should control for denomination affiliations and explore whether or not religious commitment mediates the effect of stress on dietary fat intake. This is the first study, to date, to examine the influence of religious commitment in regards to dietary fat intake.

\section{Research Question 6}

Does perceived generalized stress mediate the effect of religious commitment on dietary fat intake?

This research question was not examined in this study. There was not a direct effect between the independent variable (religious commitment) and the dependent variable (dietary fat intake) in this study. Before a mediator effect can be tested, there has to be a significant direct effect between the independent variable and the dependent variable (Baron \& Kenny, 1986; Bennett, 2000; Warner, 2013).

\section{LIMITATIONS}

Limitations of this study include the following: generalizability of the findings, study design, survey design, and accuracy and truthfulness of self-reported participant responses. Due to convenience sampling, the generalizability of the findings in this study

are limited. Cause and effect relationships cannot be determined since this study was a cross-sectional, descriptive correlational design (Polit \& Beck, 2012). The instruments used in this study to measure each variable were self-report. It is possible that participants did not answer truthfully in order to provide socially accepted responses or were unable 
to accurately recall data (e.g., height, weight, and dietary fat intake). Lastly, as mentioned in Chapter 1, it was assumed that the participants would take the online survey only once and answer the questions themselves (e.g., not have someone else complete the survey for them). However, as described in Chapter 4, 218 participants were excluded from this study mostly because of the following reasons: reported unrealistic heights and weights, took the survey more than once, and completed the survey in an unrealistic time period (i.e., completed survey in less than five minutes). Perhaps individuals, who took the survey, created a computer program that would complete the survey for them multiple times. Recommendations for such computer programs are discussed later in this chapter.

\section{STRENGTHS}

Despite these limitations, this study's strength is that it addresses the gap in the literature for emerging adults regarding the following: age overlap for emerging adults, ethnic representation for Black emerging adults, gender differences for dietary fat intake, definition of religious commitment, and overall power of this study. In most research studies, emerging adults are grouped in with other age categories, which makes it difficult to isolate data specific for ages 18-25 years. Since this study's sample was exclusively emerging adults, their data did not overlap with other age groups such as children, adolescents, and adults as seen in the literature. While this study focused solely on Black emerging adults, Blacks were underrepresented or ethnicity was not reported in some of the other studies described in Chapter 2. Some researchers have studied gender differences in fat intake, but little investigation has been done in examining those 
comparisons among emerging adults. Another strength of this study was the use of a consistent definition for religious commitment. It was defined as individuals' internal commitment to their religious beliefs (Koenig et al., 2001). Researchers tend to use religious terms interchangeably, which may make it difficult to assess which studies truly pertain to religious commitment. Lastly, the calculated power after data collection was .99 with a moderate effect size of .23 , which allowed for detection of significant relationships among the variables used in this study.

\section{NURSING IMPLICATIONS AND RECOMMENDATIONS}

\section{Implications for Practice}

The findings from this study emphasize the need for healthcare providers to provide health education on dietary behaviors to reduce one's risk for CVD, screen emerging adults for CVD risks, know the barriers emerging adults encounter in attempting to eat healthy, and consider how to incorporate the use of electronic devices in patient care for health promotion among emerging adults. In this study the participants' mean score for self-efficacy for managing their dietary fat intake was considered high (had confidence in managing their dietary fat intake); but the participants also had very high fat and saturated fat intakes. Health education on dietary behaviors may help emerging adults consider how their negative dietary behaviors can cause harm to their bodies (e.g., CVD) and seek support in changing such behaviors. A few participants with high fat screening scores and who appeared to have normal body weights made comments such as "but I'm not fat", which suggests a lack of knowledge regarding CVD 
risks or what constitutes "being fat". To reduce CVD health risks later in life, Irazusta, Hoyos, Irazusta, Ruiz, Díaz, and Gil (2007) support allocation of resources for education related to nutrition. Healthcare providers can play a key role in educating emerging adults about CVD and linking high-risk clients to community services and programs that support clients in their health behavior changes.

Along with health education, healthcare providers should screen for CVD risks during the emerging adult time-period. Some emerging adults may think they are "young and invincible"; but CVD often begins during the emerging adult years (Brown et al., 2010; Spencer, 2002). In order to prevent health problems and diagnosis them early, it is imperative to conduct such screenings among Black emerging adults, particularly among males who have higher dietary fat intakes compared to females.

As described in the literature, emerging adults encounter barriers to eating fewer high-fat foods. Some of these barriers include lack of time, cost, taste, inability to evaluate fat content of foods, and difficulty finding healthy alternatives when eating out (AbuSabha \& Achterberg, 1997; Beydoun \& Wang, 2008; Feunekes et al., 1998; Koikkalainen et al., 1999; Lloyd et al., 1995; Neumark-Sztainer et al., 1999). Barriers may vary from individual to individual. Healthcare providers should support their patients in identifying barriers to managing dietary fat intake. Identification of barriers unique to their patients may lead to tailored health promotion care plans.

These tailored health promotion care plans could be implemented through electronic devices such as cell phones and tablets. Purcell (2011) reported that younger adults, ages 18-29 years, are more likely to download and use applications on their 
electronic devices compared to older populations; and males are more likely to download applications compared to females (Purcell, Entner, \& Henderson, 2010). These health applications may help emerging adults increase their self-efficacy for managing their dietary fat intake.

Lastly, since religious commitment was high among the sample in this study, healthcare providers should consider how to create a bridge between primary care and faith-based settings (e.g., church). Researchers have reported positive health outcomes, such as improved blood pressure, increased consumption of fruits and vegetables, and increased physical activity, among participants in faith-based health promotion programs (Campbell et al., 2007; Horton, Alvear, \& Horton, 2014; Whitt-Glover, Hogan, Lang, \& Heil, 2008). In addition, in a qualitative study conducted by Horton (2013a), emerging adults shared that having an accountability partner, someone who cares about the decisions you make, incorporated into a faith-based health program may encourage individuals to practice healthy behaviors.

\section{Recommendations for Future Research}

This study is the first known study to examine factors that influence dietary fat intake among a sample of Black emerging adults. The findings from this study provide foundational knowledge to support crucial research needed on dietary behaviors among minority populations. Future researchers should consider program features of survey design, increased incentive for participants taking time to complete surveys, moderating effects of gender, instrument development for barriers, and psychoneuroimmunology. 
In this study 218 participants, who completed the online version of the survey, were excluded mostly because their reported height and weight were not logically realistic, they took the survey more than once, and the time period to complete the survey was not logically realistic (i.e., completed survey in less than five minutes). It is likely that someone created a computer program to take the survey multiple times. In order to decrease the potential for such false responses, future online surveys should be designed to include features such as the following: Completely Automated Public Turing test to tell Computers and Humans Apart (CAPTCHA) verification (a challenge response test used to determine whether a human or computer program is taking the survey), prevent ballot box stuffing (prevent individuals from taking the survey more than one time), and password protection (individuals need to enter a password to access survey) (Qualtrics, 2014). Along with adding these features, the online survey program should be designed to capture participants' birthdate and year. This current study was set up to allow participants to proceed to the survey if they were born between the years 1989-1996 at the time they took the survey. As a result, participants may have been excluded if they were age 18 but born in 1997 or age 25 but born in 1988.

Although an online research survey is cost-effective, it does require participants to invest their valued personal time. In appreciation of participants taking time to complete the survey in this study, they received a $\$ 1.29$ value gift card. An increased incentive (e.g., $\$ 10$ value gift card) should be considered for lengthy surveys, such as the one used in this study. 
In this study gender, with males having higher fat intake compared to females, was a statistically significant predictor of dietary fat intake. Future research should examine the potential moderating effect of gender in regards to social support and dietary fat intake. Scholz et al. (2013) found that gender moderated the relationship between social support and fat intake, with males who received more emotional support reporting lower fat intake. The results of this future research may guide dietary interventions that are gender-specific.

When reviewing potential instruments to use in this study, a specific instrument to measure barriers to managing one's dietary fat intake could not be found. Consequently, participants' perceived barriers for healthy eating were measured. Future research should determine what are the barriers to managing one's dietary fat intake and whether barriers for healthy eating are similar or different from barriers specific for dietary fat intake among emerging adult populations. Such future findings will add to the body of research on dietary fat intake and help researchers develop culturally sensitive interventions aimed at managing identified barriers.

Lastly, psychoneuroimmunology is the "study of how social and psychological factors affect neuroendocrine and immune functioning" (Koenig, 2002, p. 11). Future research should explore the effects of stress related to racism and biomarkers (e.g., cortisol and interleukin-6) on dietary behaviors. For Nonwhite populations, examination of stress may need to include their experiences related to race. Harrell (2000) identifies this type of stress as "racism-related stress". Perceived stress related to racism can cause health threats such as elevated blood pressure, heart rate, and cortisol levels, negative 
mental health outcomes, and suppressed immune system (Bonhomme \& Young, 2009; Paradies, 2006; Taylor \& Holden, 2009). The accumulation of perceived stress related to racism could contribute to the individual's "overall stress load", leading to wear and tear of the body (Adler \& Rehkopf, 2008; Harrell, 2000). The racial disparities in obesity prevalence (CDC, 2011) and fat intake (Wright \& Wang, 2010) may also be influenced by the biological toll of repeated exposure to stress related to racism (Adler \& Rehkopf, 2008; Mays, Cochran, \& Barnes, 2007). Blacks experience greater levels of stress related to racism compared to Whites (Chae, Lincoln, \& Jackson, 2011; Mays et al., 2007), including younger populations (Tomiyama, Puterman, Epel, Rehkopf, \& Laraia, 2013; Waelde et al., 2010). In this current study, the relationship between stress and DFI was examined. Examination of stress in another context, such as stress related to racism, should be considered for future comprehensive studies of CVD risk factors, which includes fat intake, among Black emerging adults.

\section{Recommendations for Theory Development}

The Health Promotion Model (HPM) was used in this study to examine factors that influenced dietary fat intake. As described in Chapter 1, it has been used since the early 1980s, tested among diverse populations (including emerging adults), and served as a guide to explore complex health-promoting behaviors (Pender, 1996; Pender et al., 2006). Pender's HPM worked well for this particular study in that it allowed for exploration of individual characteristics and experiences that influence behavior-specific cognitions and affect, which ultimately influence the targeted behavior outcome (Pender et al., 2006), in this case dietary fat intake. The results of this current study supported 
Pender's HPM (Figure 1). For example, according to the HPM, individual characteristics influence the behavior outcome (Pender et al., 2006). In this study, gender, an individual characteristic, predicted dietary fat intake. Behavior-specific cognitions and affect also influence the outcome in the HPM (Pender et al., 2006). In this study perceived barriers for healthy eating and perceived self-efficacy related to dietary fat intake, which are factors under this construct, were significant predictors for dietary fat intake. Therefore, the results of this study validated Pender's HPM in a sample of Black emerging adults.

Although the results from this study supported the theoretical model, future studies should test the model by including the following: 1) immediate competing demands and preferences and 2) commitment to plan of action. There was a significant positive correlation between perceived stress and gender $\left(r_{p b}=.17, p<.05\right)$, with females having higher perceived stress scores compared to males. Examination of immediate competing demands and preferences, such as role demands for females, may shed light on how participants can still practice health-promoting behaviors and balance life's demands. Based on Pender's HPM, commitment to plan of action is directly influenced by behavior-specific cognitions and affects (Pender et al., 2006), such as perceived barriers and perceived self-efficacy, which were significant predictors for dietary fat intake in this study. Results of future studies that examine these relationships with commitment to plan of action may help healthcare providers partner more efficiently with their patients in tailoring realistic individual care plans, which patients will be able to follow through. 
In Pender's HPM, immediate competing demands and preferences may directly affect health-promoting behaviors (Pender et al., 2006). However, the model does not show any relationship between behavior-specific cognitions and affects and immediate competing demands and preferences. Perhaps behavior-specific cognitions and affects, such as the significant predictor variables for dietary fat intake in this study (perceived barriers and perceived self-efficacy), directly influence immediate competing demands and preferences. Further research is needed to explore these relationships.

\section{Recommendations for Health Policy}

Future health policy recommendations include personalized prevention health plans for individuals regardless of their type of health insurance provider, establishment of faith-based health centers, and change in how health is marketed in the media. The Patient Protection and Affordable Care Act (HR 3590), which was signed into law March 2010, supports personalized prevention plan services to help reduce Medicare clients' health risks and promote self-management (The Henry J. Kaiser Family Foundation, 2013). If personalized prevention plans are effective among patients with Medicare, then these plans should be mandated to be a part of every individual's health insurance plan, whether they are enrolled in Medicare or covered by another health insurance provider. The Patient Protection and Affordable Care Act also requires all insurance providers to allow young adults to stay on their parents' health insurance plans until the age of 26 years (U.S. Department of Health and Human Services, 2014). This requirement provides an ideal opportunity for healthcare providers to address eating behaviors, coordinate nutrition counseling, and implement dietary interventions in personalized prevention 
plans for Black emerging adults covered by their parents' health insurance plans. Emerging adults tend to consume foods high in fat (Anderson, Winett, Wojcik, \& Williams, 2010), and the Black emerging adults in this current study had very high fat and saturated fat intakes. Since CVD often begins during the emerging adult years (Brown et al., 2010; Spencer, 2002), implementing personalized prevention plans may support emerging adults to adopt and maintain healthy lifestyles to reduce their risks for CVD.

In order to decrease barriers to accessing healthcare, the Patient Protection and Affordable Care Act also has provisions to award grants for school-based health centers (The Henry J. Kaiser Family Foundation, 2013). Policymakers should consider awarding such grants for faith-based health centers. Researchers have reported that religion is important among Black populations (Pew Research Center, 2009; Newport, 2006; Taylor \& Chatters, 2010), which is a similar finding in this current study. Black emerging adults in this study had high religious commitment and religiosity. In addition to possibly decreasing barriers to accessing healthcare, these faith-based health centers may facilitate culturally appropriate interventions to improve healthy eating among Black emerging adults. As described in Chapter 2, cultural food beliefs are an integral part of many extended Black family gatherings, including faith-based institutions (e.g., church). Chatters (2000) reported, “cooperation and collaboration with religious institutions have been important in contributing to the objectives of health and human services professionals in advancing the health and well-being of communities" (p. 352). The church communities could partner with faith-based health centers in developing 
innovative, culturally appropriate interventions (e.g., community-based participatory research).

Lastly, society needs to change how health is marketed in the media, particularly when targeting emerging adults. Health messages in media tend to focus on appearance, body size, and "quick fixes" (Horton, 2013a). Instead, attention should be given to the benefits of healthy behaviors (Horton, 2013a).

The expansion of personalized prevention plans beyond Medicare clients is an individual level approach. Faith-based health centers have potential to promote healthy behaviors at multiple levels. Changing how health is marketed in the media is a systems level approach. Obesity is a multifaceted problem, and policymakers and community leaders will need to take multilevel, multi-sector approaches to prevent obesity (Institute of Medicine of the National Academies, 2010).

\section{CONCLUSION}

This chapter discussed study findings from Chapter 4 in relationship to the literature. Gender (high fat intake among males), perceived barriers for healthy eating, and perceived self-efficacy related to dietary fat intake were significant predictors for participants' dietary fat intake, with gender having the greatest effect. Religious commitment influenced the effects of perceived stress on dietary fat intake.

The description of the findings in relationship to the literature leads into this chapter's discussions on strengths and limitations of the study. Despite the noted limitations of this study, it addresses several gaps in the literature regarding dietary fat 
intake among Black emerging adults. This is the first known study to examine factors influencing dietary fat intake among a sample of Black emerging adults.

This chapter concluded with discussions on implications and recommendations for practice, research, theory, and health policy. Findings from this study may help researchers, policymakers, and healthcare providers develop culturally, age-appropriate, and gender-specific interventions to help Black emerging adults decrease their dietary fat intake and risks for CVD. In addition, these interventions may support Black emerging adults adopt and maintain healthy lifestyles well into their older adult years. 
Appendices 


\section{Appendix A Cover Letter}

Date

Dear

My name is Shalonda Horton from The University of Texas at Austin. I am a graduate student working on my $\mathrm{PhD}$ in nursing. I am writing to request your help in recruiting participants to take a survey for my research study.

The purpose of this study is to examine factors that may influence dietary fat intake among Black young adults. The study involves completing an anonymous online survey, which takes about 15 minutes to complete. None of the participants' responses can be linked to their identity, so they are encouraged to answer honestly. The questions consist of items such as, but not limited to, the following:

- Demographic information: age, marital status, and highest education level completed

- Cultural information: religious commitment

- Barriers related to eating healthy

- Social support in eating healthy

- Stress

- Dietary fat intake

To participate in the study participants need to: live in the United States, self-identify as African American or Black, be 18 to 25 years old, and if female, not be pregnant.

About 220 individuals will be completing this survey. There are no foreseeable risks to participating in this study. There are no known benefits to participating in this study. Participation in this study may increase one's awareness of his/her own health behaviors.

There are no costs associated with this study. There is only the inconvenience of the time that it takes to complete the survey. Participants will need access to a computer and Internet, which is available for free at their local library.

After completing the online survey, participants can enter drawings for a chance to receive an electronic tablet and one of eleven $\$ 10$ value gift cards.

With your permission, I would like to come to one of your (name of organization/business) meetings to briefly share information about my study.

I have also included the web link to the online survey and a flyer to share with your (name of organization/business) members/patrons: (insert link to survey). 
Thank you in advance for helping me to examine factors that may influence dietary fat intake.

Sincerely,

(insert investigator's name, credentials, and university) 


\section{Appendix B Consent}

\section{Consent to Participate in Internet Research}

\section{Identification of Investigator and Purpose of Study}

You are invited to participate in a research study, entitled "Factors Influencing Dietary Fat Intake Among Black Young Adults." The study is being conducted by Shalonda Horton in the School of Nursing of The University of Texas at Austin, 1710 Red River, Austin, Texas 78701, 512-4758694 (office number), dietaryfatresearchstudy@gmail.com.

The purpose of this online research study is to examine factors that may influence dietary fat intake. Your participation in the study will contribute to a better understanding of what influences dietary fat intake, an important risk factor for heart disease and weight gain. You are free to contact the investigator at the above address, phone number, and/or email address to discuss the study. In order to participate in this study, you must be 18 to 25 years old, self-identify as Black, and speak and read English. You will be excluded if you are pregnant, due to your special nutritional requirements, or live outside of the U.S.

If you agree to participate:

- You, and not someone else (e.g., friend or family member), agree to complete a survey

- You agree to complete the survey only once and not multiple times (one completed survey per person)

- The confidential online survey will take approximately 30 minutes of your time to complete.

- You will complete a survey with questions that assess the following information

○ Demographic information such as your age, marital status, and highest education level completed

- Cultural information such as your religious commitment

- Barriers related to eating healthy

- Social support in eating healthy

- Stress

○ Dietary fat intake

- The survey will be open until 220 participants have completed the study. To thank you for your time in completing the survey, you may enter a drawing for the chance to win an electronic tablet and one of eleven $\$ 10$ Amazon.com gift cards. Winners will be notified by the email address they provide at the end of the survey. The drawing will be held after the recruitment goal has been met.

\section{Risks/Benefits/Confidentiality of Data}

There are no known risks to participating in this study. If the survey questions make you feel uncomfortable, you may stop participating in the study, and a list of mental health resources is available upon request at the end of the survey. There are no benefits to participating in this study. At your request, your dietary fat screening intake will be sent to you via the email address you provide at the end of the survey. There will be no costs for participating in this study. Your name and email address will be kept during the data collection phase for tracking purposes only. This study is designed to keep your name and 
email separate from your survey responses so there is no information that can be linked to you. Once the study is completed, your name and email will be destroyed. A limited number of research team members will have access to the data during data collection.

\section{Participation or Withdrawal}

Your participation in this study is voluntary. You may decline to answer any question and you have the right to withdraw from participation at any time. Withdrawal will not affect your relationship with The University of Texas in anyway. If you do not want to participate either simply stop participating or close the browser window for the survey. For incomplete surveys, two automatic email reminders will be sent to you to complete the survey.

\section{Contacts}

If you have any questions about the study, contact one of the following researchers: Shalonda Horton, MSN, RN, at (512) 475-8694 (office number) or send an email to dietaryfatresearchstudy@gmail.com or Gayle Timmerman, PhD, RN, at (512) 471-9087 (office number) and/or gtimmerman@mail.utexas.edu. This study has been reviewed by The University of Texas at Austin Institutional Review Board and the study number is [STUDY NUMBER].

\section{Questions about your rights as a research participant.}

If you have questions about your rights or are dissatisfied at any time with any part of this study, you can contact, anonymously if you wish, the Institutional Review Board by phone at (512) 471-8871 or email at orsc@uts.cc.utexas.edu.

If you agree to participate in this research study, you will give consent below, click continue, and follow the instructions provided.

Thank you.

\section{Please print a copy of this document (consent) for your records.}

If they do or do not want to participate in the online research study, they will click on one of the

following buttons.

Please check one of the boxes below (consent).

Yes, I read and understand the consent. I agree to participate in this study. I do not agree to participate in this study. 


\section{Appendix C Instruments}

\section{Factors Influencing Dietary Fat Intake Among Black Emerging Adults}

\section{Consent to Participate in Internet Research}

\section{Identification of Investigator and Purpose of Study}

You are invited to participate in a research study, entitled "Factors Influencing Dietary Fat Intake Among Black Young Adults (ages 18 to 25 years)." The study is being conducted by Shalonda Horton in the School of Nursing of The University of Texas at Austin, 1710 Red River, Austin, Texas 78701, 512-475-8694 (office number), dietaryfatresearchstudy@gmail.com.

The purpose of this online research study is to examine factors that may influence dietary fat intake. Your participation in the study will contribute to a better understanding of what influences dietary fat intake, an important risk factor for heart disease and weight gain. You are free to contact the investigator at the above address, phone number, and/or email address to discuss the study. In order to participate in this study, you must be 18 to 25 years old, self-identify as Black, and speak and read English. You will be excluded if you are pregnant, due to your special nutritional requirements, or live outside of the U.S.

If you agree to participate:

- You, and not someone else (e.g., friend or family member), agree to complete a survey

- You agree to complete the survey only once and not multiple times (one completed survey per person)

- The confidential online survey will take approximately 15 to 25 minutes of your time to complete.

- You will complete a survey with questions that assess the following information

- Demographic information such as your age, marital status, and highest education level completed

- Cultural information such as your level of spirituality

- Barriers related to eating healthy

- Social support in eating healthy

- Stress

- Dietary fat intake

- This survey will be open until 220 participants have completed the study. To thank you for your time in completing the survey, you will be sent a $\$ 1.29$ value gift card to Amazon.com to use for a music download. The $\$ 1.29$ value electronic gift card will be sent to you via the email you provide in the survey. However, 
participants will be disqualified to receive the $\$ 1.29$ value gift card if their survey is not accepted for one of the following reasons: 1) participant lives outside of the United States; 2 ) participant's reported height and weight are not logically realistic (e.g., 2 feet and 165 pounds); 3) more than one survey is completed by the participant (e.g., same IP address and/or latitude and longitude area); and/or the time period to complete the survey is not logically realistic.

\section{Risks/Benefits/Confidentiality of Data}

There are no known risks to participating in this study. If the survey questions make you feel uncomfortable, you may stop participating in the study, and a list of mental health resources is available upon request at the end of the survey. There are no benefits to participating in this study. At your request, your dietary fat screening intake results will be sent to you via the email address you provide at the beginning of the survey. There will be no costs for participating in this study. Your email address will be kept during the data collection phase for tracking purposes only. During data analyses your email address will not be included with your survey responses, so there is no information that can be linked to you. Once the study is completed, your email will be destroyed. A limited number of research team members will have access to the data during data collection and analysis.

\section{Participation or Withdrawal}

Your participation in this study is voluntary. You may decline to answer any question and you have the right to withdraw from participation at any time. Withdrawal will not affect your relationship with The University of Texas in anyway. If you do not want to participate either simply stop participating or close the browser window for the survey. For incomplete surveys, two automatic email reminders will be sent to you to complete the survey.

\section{Contacts}

If you have any questions about the study, contact one of the following researchers: Shalonda Horton, MSN, RN, at (512) 475-8694 (office number) or send an email to dietaryfatresearchstudy@gmail.com or Gayle Timmerman, PhD, RN, at (512) 4719087 (office number) and/or gtimmerman@mail.utexas.edu. This study has been reviewed by The University of Texas at Austin Institutional Review Board and the study number is 2013-06-0014.

\section{Questions about your rights as a research participant.}

If you have questions about your rights or are dissatisfied at any time with any part of this study, you can contact, anonymously if you wish, the Institutional Review Board by phone at (512) 471-8871 or email at orsc@uts.cc.utexas.edu.

If you agree to participate in this research study, you will give consent below, click continue, and follow the instructions provided. 
Thank you.

Please print a copy of this document (consent) for your records.

Please select one of the choices below (consent).

O Yes, I read and understand the consent. I agree to participate in this study.

O I do not agree to participate in this study.

Do you read and speak English?

O Yes, I do read and speak English

O No, I do not read and speak English

In what year were you born?

O Between the years 1989-1996

O Other

Do you live in the United States?

O Yes, I live in the United States

No, I do not live in the United States

Do you self-identify as African American or Black?

O Yes, I self-identify as African American or Black

O No, I do not self-identify as African American or Black

Are you pregnant?

O Yes

O No

Use the $<<$ and $>>$ buttons to move backwards and forwards through the survey. If you start the survey and decide to finish it at a later time, your answers on the previous pages will be saved automatically. However, when you come back to the survey be sure to use the same device (e.g., computer, smartphone, tablet) and browser as previously used because the save feature is linked to a cookie. 
Your email address will be used only to determine if you have or have not completed the survey. Your email address will be destroyed at the completion of the research study.

What is your email address?

Example: dietaryfatresearchstudy@gmail.com

How old are you? Please provide your age in years.

What is your gender?

O Male

O Female

Do you consider yourself to be multiethnic (e.g., Black and White)?

O Yes

O No

How tall are you without shoes? (to the nearest $1 / 2$ inch)

Example: 5 feet 3.5 inches

How much do you weigh without shoes? (to the nearest pound)

Example: 165 pounds

When was the last time you weighed yourself? (MM/DD/YYYY format) Example: $12 / 30 / 2013$ 
How often do you check your weight?

O Daily

Once a week

O Twice a month

O Once a month

Once every 6 months

O Once a year

O I have never checked my weight

How would you consider your weight?

Very underweight

S Somewhat underweight

O Just about right

O Somewhat overweight

O Very overweight

O I don't know

What is the highest grade level of school you have completed?

6th grade or less

7th grade

8th grade

9th grade

O 10th grade

O 11th grade

O General Equivalency (GED)

O High school diploma

O Some college

O Associate Degree

O Bachelor's Degree

Graduate Degree (e.g., Master's, MD, JD)

What is your marital status?

O Single

O Married

O Living with partner but not married

O Divorced

Widowed 
How many years have you lived in the United States?

Example: 10 years

What state do you currently live in (e.g., Texas)?

What is your living arrangement?

O I live alone

O I live with my parents

O I live with my spouse/partner

O I live with a roommate (e.g., friend)

O Other (please specify)

How many children do you have?

O None

O 1

○ 2

O 3

O 4

O 5 or more

If you have children, do they live with you?

$\mathrm{O}$ Yes

O No

O Not applicable

What is your employment status?

O Working full-time paid employment

O Working part-time paid employment

O Self-employed

O Unemployed

Other (please specify) 
What prevents or gets in the way of you trying to manage your fat intake (food barriers in eating a low-fat diet)? 
The below questions are from the Block Dietary Fat Screener. The reference for these questions is the following: Block, G., Gillespie, C., Rosenbaum, E. H., \& Jenson, C. (2000). A rapid food screener to assess fat and fruit and vegetable intake. American Journal of Preventive Medicine, 18(4), 284-288.

Think about your eating habits over the past year or so. About how often do you eat each of the following foods? Remember breakfast, lunch, dinner, snacks and eating out. Mark one bubble for each food.

\begin{tabular}{|c|c|c|c|c|c|}
\hline & $\begin{array}{l}1 \text { time a } \\
\text { MONTH or } \\
\text { less }\end{array}$ & $\begin{array}{l}2-3 \text { times a } \\
\text { MONTH }\end{array}$ & $\begin{array}{l}1 \text { - } 2 \text { times a } \\
\text { WEEK }\end{array}$ & $\begin{array}{l}\text { 3-4 times a } \\
\text { WEEK }\end{array}$ & $\begin{array}{l}5+\text { times a } \\
\text { WEEK }\end{array}$ \\
\hline $\begin{array}{l}\text { Hamburgers, } \\
\text { ground beef, } \\
\text { meat burritos, } \\
\text { tacos }\end{array}$ & 0 & 0 & 0 & 0 & 0 \\
\hline $\begin{array}{l}\text { Beef or pork, } \\
\text { such as } \\
\text { steaks, roasts, } \\
\text { ribs, or in } \\
\text { sandwiches }\end{array}$ & 0 & 0 & 0 & 0 & 0 \\
\hline Fried chicken & 0 & 0 & 0 & 0 & 0 \\
\hline $\begin{array}{l}\text { Hot dogs, or } \\
\text { Polish or } \\
\text { Italian } \\
\text { sausage }\end{array}$ & 0 & 0 & 0 & 0 & 0 \\
\hline $\begin{array}{l}\text { Cold cuts, } \\
\text { lunch meats, } \\
\text { ham (not } \\
\text { low-fat) }\end{array}$ & 0 & 0 & 0 & 0 & 0 \\
\hline $\begin{array}{l}\text { Bacon or } \\
\text { breakfast } \\
\text { sausage }\end{array}$ & 0 & 0 & 0 & 0 & 0 \\
\hline $\begin{array}{l}\text { Salad } \\
\text { dressings (not } \\
\text { low-fat) }\end{array}$ & 0 & 0 & 0 & 0 & 0 \\
\hline
\end{tabular}




\begin{tabular}{|c|c|c|c|c|c|}
\hline $\begin{array}{l}\text { Margarine, } \\
\text { butter or } \\
\text { mayo on } \\
\text { bread or } \\
\text { potatoes }\end{array}$ & $\mathrm{O}$ & O & $\mathrm{O}$ & $\mathrm{O}$ & $\mathrm{O}$ \\
\hline $\begin{array}{l}\text { Margarine, } \\
\text { butter or oil } \\
\text { in cooking }\end{array}$ & $\mathrm{O}$ & $\mathrm{O}$ & $\mathrm{O}$ & $\mathrm{O}$ & $\mathrm{O}$ \\
\hline $\begin{array}{l}\text { Eggs (not } \\
\text { Egg Beaters } \\
\text { or just egg } \\
\text { whites) }\end{array}$ & $\mathrm{O}$ & $\mathrm{O}$ & $\mathrm{O}$ & $\mathrm{O}$ & $\mathrm{O}$ \\
\hline Pizza & $\mathrm{O}$ & 0 & 0 & 0 & 0 \\
\hline $\begin{array}{l}\text { Cheese, } \\
\text { cheese spread } \\
\text { (not low-fat) }\end{array}$ & $\mathrm{O}$ & $\mathrm{O}$ & $\mathrm{O}$ & $\mathrm{O}$ & $\mathrm{O}$ \\
\hline Whole milk & 0 & O & $\mathrm{O}$ & $\mathrm{O}$ & $\mathrm{O}$ \\
\hline $\begin{array}{l}\text { French fries, } \\
\text { fried potatoes }\end{array}$ & 0 & $\mathrm{O}$ & 0 & O & 0 \\
\hline $\begin{array}{l}\text { Corn chips, } \\
\text { potato chips, } \\
\text { popcorn, } \\
\text { crackers }\end{array}$ & $\mathrm{O}$ & $\mathrm{O}$ & $\mathrm{O}$ & $\mathrm{O}$ & $\mathrm{O}$ \\
\hline $\begin{array}{l}\text { Doughnuts, } \\
\text { pastries, } \\
\text { cake, cookies } \\
\text { (not low-fat) }\end{array}$ & $\mathrm{O}$ & $\mathrm{O}$ & $\mathrm{O}$ & $\mathrm{O}$ & $\mathrm{O}$ \\
\hline $\begin{array}{l}\text { Ice cream } \\
\text { (not sherbet } \\
\text { or non-fat) }\end{array}$ & $\mathrm{O}$ & 0 & $\mathrm{O}$ & $\mathrm{O}$ & $\mathrm{O}$ \\
\hline
\end{tabular}


The below questions/statements are from the Duke University Religion Index. The reference for these questions/statements is the following: Koenig, H. G., Meador, K., \& Parkerson, G. (1997). Religion index for psychiatric research: A 5-item measure for use in health outcome studies. American Journal of Psychiatry, 154, 885-886.

How often do you attend church or other religious meetings?

O More than once/week

O Once a week

O A few times a month

A few times a year

Once a year or less

O Never

The following 3 statements are about religious belief or experience. Please mark the extent to which each statement is true or not true for you.

\begin{tabular}{|c|c|c|c|c|c|}
\hline & $\begin{array}{l}\text { Definitely } \\
\text { true of me }\end{array}$ & $\begin{array}{l}\text { Tends to be } \\
\text { true }\end{array}$ & Unsure & $\begin{array}{l}\text { Tends not } \\
\text { to be true }\end{array}$ & $\begin{array}{l}\text { Definitely } \\
\text { not true }\end{array}$ \\
\hline $\begin{array}{l}\text { In my life, I } \\
\text { experience } \\
\text { the presence } \\
\text { of the Divine } \\
\text { (i.e., God). }\end{array}$ & 0 & O & 0 & $\mathrm{O}$ & 0 \\
\hline $\begin{array}{l}\text { My religious } \\
\text { beliefs are } \\
\text { what really } \\
\text { lie behind } \\
\text { my whole } \\
\text { approach to } \\
\text { life. }\end{array}$ & $\mathrm{O}$ & $\mathrm{O}$ & 0 & $\mathrm{O}$ & O \\
\hline $\begin{array}{l}\text { I try hard to } \\
\text { carry my } \\
\text { religion over } \\
\text { into all other } \\
\text { dealings in } \\
\text { life. }\end{array}$ & $\mathrm{O}$ & $\mathrm{O}$ & $\mathrm{O}$ & $\mathrm{O}$ & $\mathrm{O}$ \\
\hline
\end{tabular}


The below questions are from the Economic Adequacy Scale. The reference for these questions is the following: Lobo, M. (personal communication, February 11, 2013).

The following are general questions related to the economics of daily living. I want to know if YOUR income allows you to meet YOUR NEEDS. Please circle your answer.

\begin{tabular}{|c|c|c|c|c|}
\hline & $\begin{array}{l}\text { More than } \\
\text { adequate }\end{array}$ & Adequately & $\begin{array}{l}\text { Less than } \\
\text { adequate }\end{array}$ & Not at all \\
\hline $\begin{array}{l}\text { Does your } \\
\text { income allow } \\
\text { you to meet } \\
\text { your needs for } \\
\text { daily living }\end{array}$ & 0 & O & 0 & O \\
\hline $\begin{array}{l}\text { Does your } \\
\text { income allow } \\
\text { you to meet } \\
\text { your rent or } \\
\text { mortgage } \\
\text { payment }\end{array}$ & O & O & O & $\mathrm{O}$ \\
\hline $\begin{array}{l}\text { Does your } \\
\text { income allow } \\
\text { you to meet } \\
\text { your food bills }\end{array}$ & 0 & O & O & O \\
\hline $\begin{array}{l}\text { Does your } \\
\text { income allow } \\
\text { you to meet } \\
\text { your health care } \\
\text { needs }\end{array}$ & O & O & O & O \\
\hline $\begin{array}{l}\text { Does your } \\
\text { income allow } \\
\text { you to } \\
\text { participate in } \\
\text { recreation }\end{array}$ & 0 & O & 0 & O \\
\hline $\begin{array}{l}\text { Does your } \\
\text { income allow } \\
\text { you to meet } \\
\text { baby costs }\end{array}$ & O & O & O & O \\
\hline
\end{tabular}




\begin{tabular}{|c|c|c|c|c|}
\hline $\begin{array}{l}\text { Does your } \\
\text { income allow } \\
\text { you to meet } \\
\text { other financial } \\
\text { needs you have }\end{array}$ & 0 & $\mathrm{O}$ & $\mathrm{O}$ & 0 \\
\hline
\end{tabular}

Specify below: 
The below questions are from the Healthy Foods and Snacks-Barriers Subscale. The reference for these questions is the following: Tucker, C. M., Rice, K. G., Hou, W., Kaye, L. B., Nolan, S. E. M., Grandoit, D. J., ... Desmond, F. F. (2011). Development of the Motivators of and Barriers to Health-Smart Behaviors Inventory. Psychological Assessment, 23(2), 487-503. doi: 10.1037/a0022299

Indicate how much you agree or disagree with each of the following statements. When I do not eat healthy foods (like fruits, vegetables, and lower-calorie snacks), it is because...

\begin{tabular}{|c|c|c|c|c|}
\hline & $\begin{array}{l}\text { Strongly } \\
\text { disagree }\end{array}$ & $\begin{array}{l}\text { Somewhat } \\
\text { disagree }\end{array}$ & $\begin{array}{l}\text { Somewhat } \\
\text { agree }\end{array}$ & Strongly agree \\
\hline $\begin{array}{l}\text { when there are } \\
\text { unhealthy foods } \\
\text { at home, it is } \\
\text { hard to choose } \\
\text { healthy foods. }\end{array}$ & 0 & 0 & O & 0 \\
\hline $\begin{array}{l}\text { when I think } \\
\text { "healthy food", } \\
\text { I think "tastes } \\
\text { bad." }\end{array}$ & 0 & $\mathrm{O}$ & $\mathrm{O}$ & $\mathrm{O}$ \\
\hline $\begin{array}{l}\text { fresh healthy } \\
\text { foods are not } \\
\text { easily available. }\end{array}$ & 0 & $\mathrm{O}$ & $\mathrm{O}$ & $\mathrm{O}$ \\
\hline $\begin{array}{l}\text { when I go to the } \\
\text { grocery store, I } \\
\text { do not } \\
\text { specifically } \\
\text { think about } \\
\text { buying fruits } \\
\text { and vegetables. }\end{array}$ & 0 & O & O & $\mathrm{O}$ \\
\hline $\begin{array}{l}\text { I get cravings } \\
\text { for unhealthy } \\
\text { foods. }\end{array}$ & 0 & $\mathrm{O}$ & $\mathrm{O}$ & 0 \\
\hline $\begin{array}{l}\text { I do not like the } \\
\text { taste of most }\end{array}$ & 0 & $\mathrm{O}$ & $\mathrm{O}$ & 0 \\
\hline & O & 0 & 0 & 0 \\
\hline
\end{tabular}




\begin{tabular}{|c|c|c|c|c|}
\hline $\begin{array}{l}\text { I cannot get } \\
\text { healthy snacks } \\
\text { in the snack } \\
\text { machines. }\end{array}$ & & & & \\
\hline $\begin{array}{l}\text { I do not like to } \\
\text { try new fruits or } \\
\text { vegetables that } \\
\text { I have never } \\
\text { had before. }\end{array}$ & 0 & 0 & 0 & 0 \\
\hline $\begin{array}{l}\text { when someone } \\
\text { cooks or gives } \\
\text { me unhealthy } \\
\text { food, I eat it. }\end{array}$ & 0 & 0 & 0 & 0 \\
\hline $\begin{array}{l}\text { I do not look or } \\
\text { feel any } \\
\text { different when I } \\
\text { eat healthy. }\end{array}$ & 0 & 0 & 0 & 0 \\
\hline $\begin{array}{l}\text { I crave sweets } \\
\text { or junk food } \\
\text { instead of fruit } \\
\text { as a snack. }\end{array}$ & 0 & 0 & 0 & 0 \\
\hline $\begin{array}{l}\text { I just do not } \\
\text { care about } \\
\text { eating fruits and } \\
\text { vegetables } \\
\text { every day. }\end{array}$ & 0 & 0 & 0 & 0 \\
\hline $\begin{array}{l}\text { when I make or } \\
\text { buy a meal, I do } \\
\text { not think about } \\
\text { whether or not } \\
\text { it has fruits or }\end{array}$ & 0 & 0 & 0 & 0 \\
\hline $\begin{array}{l}\text { healthy foods } \\
\text { are not easy to } \\
\text { find at }\end{array}$ & 0 & 0 & 0 & 0 \\
\hline
\end{tabular}




\begin{tabular}{|c|c|c|c|c|}
\hline restaurants. & & & & \\
\hline $\begin{array}{l}\text { I just do not } \\
\text { care about } \\
\text { eating healthy } \\
\text { every day. }\end{array}$ & $O$ & 0 & $\mathrm{O}$ & $\mathrm{O}$ \\
\hline
\end{tabular}


The next section of questions are from the Resisting Relapse Subscale.

The reference for these questions is the following: Sallis, J. F., Pinski, R.B., Grossman, R. M., Patterson, T. L., \& Nader, Philip R. (1988). The development of self-efficacy scales for health-related diet and exercise behaviors. Health Education Research, 3(3), 283-292. doi: 10.1093/her/3.3.283

Below is a list of things people might do while trying to change their eating habits. I am mainly interested in salt and fat intake, rather than weight reduction. Whether you are trying to change your eating habits or not, please rate how confident you are that you could really motivate yourself to do things like these consistently, for at least six months. Please select one response (ranging from "sure I could not do it" to "sure I could do it") for each item on: How sure are you that you can do these things?

\begin{tabular}{|c|c|c|c|c|c|c|}
\hline & $\begin{array}{l}\text { Sure I } \\
\text { could not } \\
\text { do it }\end{array}$ & & $\begin{array}{l}\text { Maybe I } \\
\text { could do it }\end{array}$ & & $\begin{array}{l}\text { Sure I } \\
\text { could do it }\end{array}$ & $\begin{array}{l}\text { Does not } \\
\text { apply }\end{array}$ \\
\hline $\begin{array}{l}\text { Stick to } \\
\text { low-fat, } \\
\text { low-salt } \\
\text { foods when } \\
\text { you feel } \\
\text { depressed, } \\
\text { bored, or } \\
\text { tense }\end{array}$ & 0 & 0 & O & 0 & O & 0 \\
\hline $\begin{array}{l}\text { Stick to } \\
\text { low-fat, } \\
\text { low-salt } \\
\text { foods when } \\
\text { there is } \\
\text { high-fat, } \\
\text { high-salt } \\
\text { food } \\
\text { readily } \\
\text { available at } \\
\text { a party }\end{array}$ & 0 & $\mathrm{O}$ & O & 0 & O & 0 \\
\hline $\begin{array}{l}\text { Stick to } \\
\text { low-fat, } \\
\text { low-salt } \\
\text { foods when } \\
\text { dining with }\end{array}$ & O & $\mathrm{O}$ & O & 0 & O & 0 \\
\hline
\end{tabular}




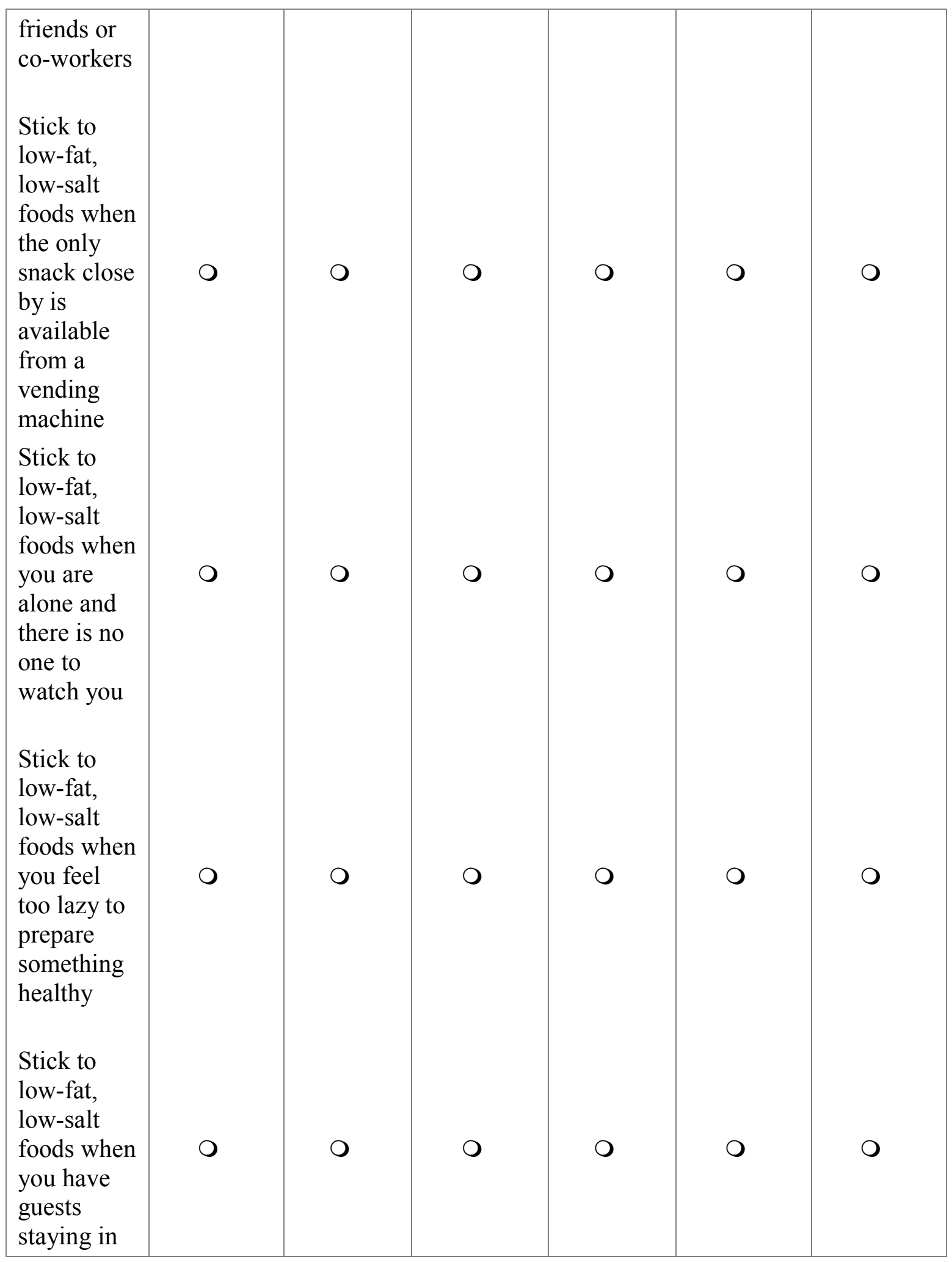




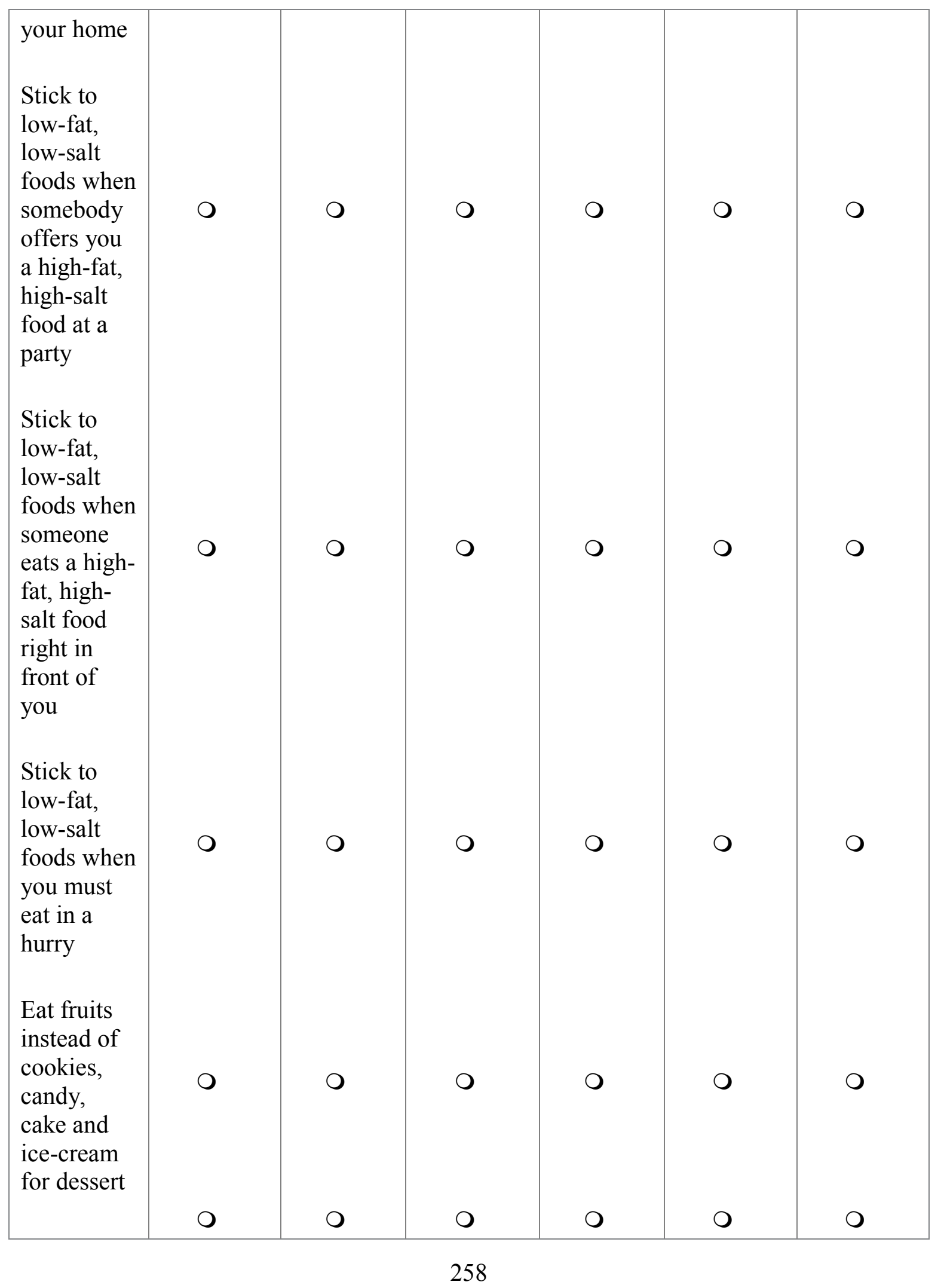




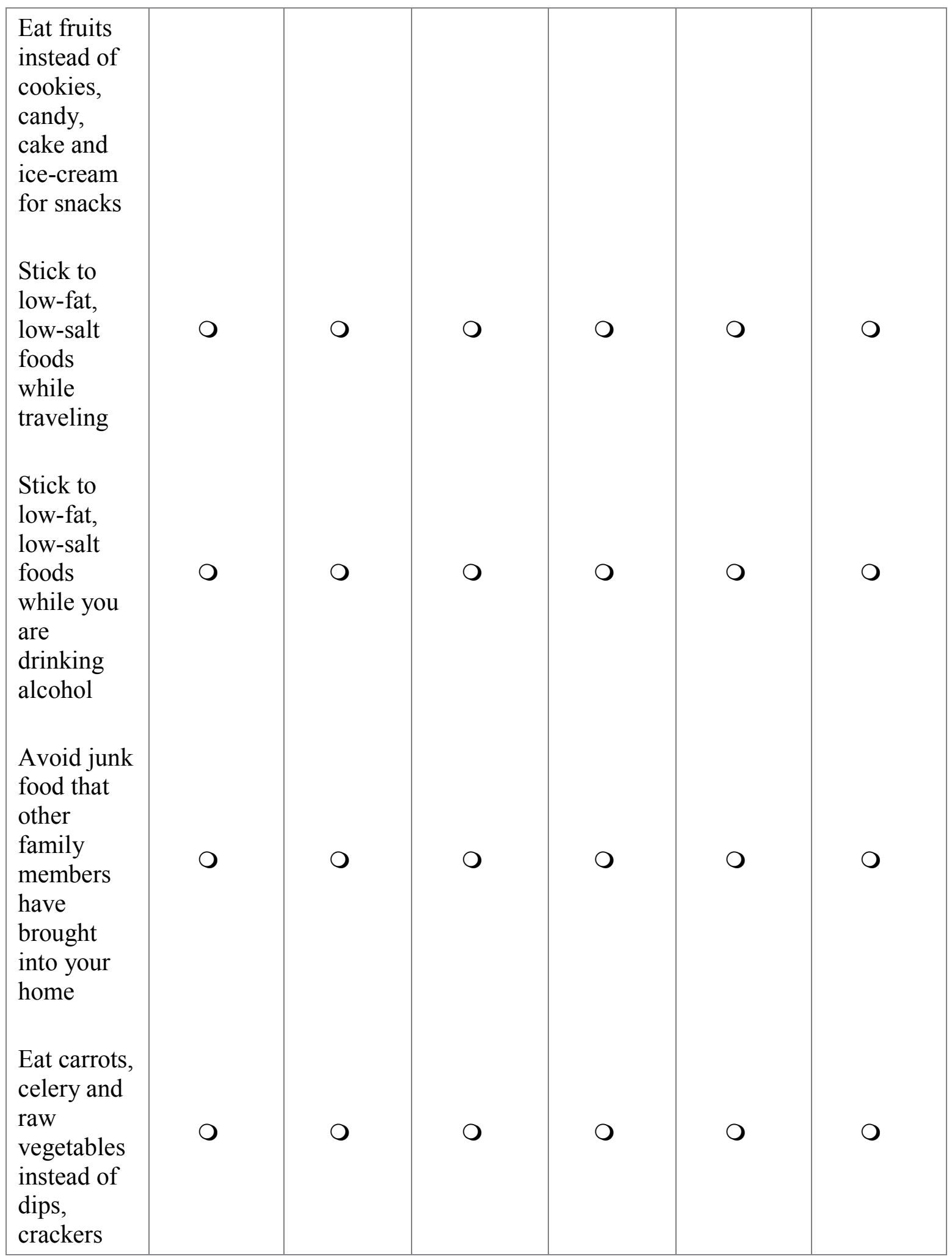




\begin{tabular}{|c|c|c|c|c|c|c|}
\hline $\begin{array}{l}\text { and potato } \\
\text { chips for } \\
\text { snacks }\end{array}$ & & & & & & \\
\hline $\begin{array}{l}\text { Avoid } \\
\text { eating fast } \\
\text { food for } \\
\text { meals }\end{array}$ & $\mathrm{O}$ & 0 & 0 & O & 0 & 0 \\
\hline
\end{tabular}


The below questions are from the Family Support for Heart Healthy Eating Habits Scale.

The reference for these questions is the following: Sallis, J. F., Grossman, R. M., Pinski, R.B, Patterson, T. L., \& Nader, Philip R. (1987). The development of scales to measure social support for diet and exercise behaviors. Preventive Medicine, 16, 825-836.

Below is a list of things family members might do or say to someone who is trying to improve their eating habits. I am interested in high fat and high salt (or high sodium) foods. If you are not trying to make any of these dietary changes, then some of the questions may not apply to you, but please read and give an answer to every question. Rate how often your family has said or done what is described in the statements below during the last three months.

\begin{tabular}{|c|c|c|c|c|c|}
\hline & None & Rarely & A few times & Often & Very often \\
\hline $\begin{array}{l}\text { Encouraged } \\
\text { me not to eat } \\
\text { high-salt, } \\
\text { high-fat } \\
\text { foods when } \\
\text { I'm tempted } \\
\text { to do so. }\end{array}$ & 0 & 0 & 0 & 0 & 0 \\
\hline $\begin{array}{l}\text { Discussed } \\
\text { my eating } \\
\text { habit changes } \\
\text { with me. }\end{array}$ & 0 & 0 & 0 & 0 & 0 \\
\hline $\begin{array}{l}\text { Reminded } \\
\text { me not to eat } \\
\text { high-salt, } \\
\text { high-fat } \\
\text { foods. }\end{array}$ & 0 & 0 & 0 & 0 & 0 \\
\hline $\begin{array}{l}\text { Asked me for } \\
\text { ideas on how } \\
\text { they can eat } \\
\text { healthier } \\
\text { diets. }\end{array}$ & 0 & 0 & 0 & 0 & 0 \\
\hline Offered me & 0 & 0 & 0 & 0 & 0 \\
\hline
\end{tabular}




\begin{tabular}{|c|c|c|c|c|c|}
\hline $\begin{array}{l}\text { low-salt, low- } \\
\text { fat snacks } \\
\text { when I visit } \\
\text { in their } \\
\text { homes. }\end{array}$ & & & & & \\
\hline $\begin{array}{l}\text { Commented } \\
\text { if I went back } \\
\text { to eating } \\
\text { high-salt, } \\
\text { high-fat } \\
\text { foods. }\end{array}$ & 0 & 0 & 0 & 0 & 0 \\
\hline $\begin{array}{l}\text { Ate high-salt } \\
\text { or high-fat } \\
\text { foods in front } \\
\text { of me. }\end{array}$ & 0 & 0 & 0 & 0 & 0 \\
\hline $\begin{array}{l}\text { Brought } \\
\text { home high- } \\
\text { salt, high-fat } \\
\text { foods. }\end{array}$ & 0 & 0 & 0 & 0 & 0 \\
\hline $\begin{array}{l}\text { Offered me } \\
\text { high-salt, } \\
\text { high-fat } \\
\text { foods. }\end{array}$ & 0 & 0 & 0 & 0 & 0 \\
\hline $\begin{array}{l}\text { Requested } \\
\text { high-salt, } \\
\text { high-fat } \\
\text { foods. }\end{array}$ & 0 & 0 & 0 & 0 & 0 \\
\hline $\begin{array}{l}\text { Criticized the } \\
\text { food I eat. }\end{array}$ & 0 & 0 & 0 & 0 & 0 \\
\hline $\begin{array}{l}\text { Refused to } \\
\text { eat the } \\
\text { healthy foods } \\
\text { I prepared. }\end{array}$ & 0 & 0 & 0 & 0 & 0 \\
\hline
\end{tabular}




\begin{tabular}{|c|c|c|c|c|c|}
\hline $\begin{array}{l}\text { Told me if I } \\
\text { keep eating } \\
\text { low-fat foods } \\
\text { I'll get to be } \\
\text { too skinny. }\end{array}$ & 0 & 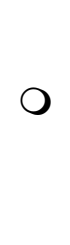 & 0 & 0 & 0 \\
\hline
\end{tabular}


The next section of questions are from the Friend Support for Heart Healthy Eating Habits Scale.

The reference for these questions is the following: Sallis, J. F., Grossman, R. M., Pinski, R.B, Patterson, T. L., \& Nader, Philip R. (1987). The development of scales to measure social support for diet and exercise behaviors. Preventive Medicine, 16, 825-836.

Below is a list of things friends, acquaintances, or coworkers might do or say to someone who is trying to improve their eating habits. I am interested in high fat and high salt (or high sodium) foods. If you are not trying to make any of these dietary changes, then some of the questions may not apply to you, but please read and give an answer to every question. Rate how often your friends, acquaintances, or coworkers have said or done what is described in the statements below during the last three months.

\begin{tabular}{|c|c|c|c|c|c|}
\hline & None & Rarely & A few times & Often & Very often \\
\hline $\begin{array}{l}\text { Reminded me } \\
\text { not to eat } \\
\text { high-salt, } \\
\text { high-fat foods. }\end{array}$ & 0 & 0 & 0 & 0 & 0 \\
\hline $\begin{array}{l}\text { Encouraged } \\
\text { me not to eat } \\
\text { high-salt, } \\
\text { high-fat foods } \\
\text { when I'm } \\
\text { tempted to do } \\
\text { so. }\end{array}$ & 0 & 0 & 0 & 0 & 0 \\
\hline $\begin{array}{l}\text { Commented if } \\
\text { I went back to } \\
\text { eating high- } \\
\text { salt, high-fat } \\
\text { foods. }\end{array}$ & 0 & 0 & 0 & 0 & 0 \\
\hline $\begin{array}{l}\text { Complimented } \\
\text { me about } \\
\text { changing my } \\
\text { eating habits. }\end{array}$ & 0 & 0 & 0 & 0 & 0 \\
\hline $\begin{array}{l}\text { Discussed my } \\
\text { eating habit } \\
\text { changes with }\end{array}$ & 0 & 0 & 0 & 0 & 0 \\
\hline
\end{tabular}




\begin{tabular}{|c|c|c|c|c|c|}
\hline me. & & & & & \\
\hline $\begin{array}{l}\text { Made negative } \\
\text { comments } \\
\text { when I went } \\
\text { back to eating } \\
\text { high-salt, } \\
\text { high-fat foods. }\end{array}$ & 0 & 0 & 0 & 0 & 0 \\
\hline $\begin{array}{l}\text { Complained } \\
\text { that I spent } \\
\text { too much } \\
\text { money on } \\
\text { low-salt, low- } \\
\text { fat foods. }\end{array}$ & 0 & 0 & 0 & 0 & 0 \\
\hline $\begin{array}{l}\text { Refused to eat } \\
\text { the same } \\
\text { foods I eat. }\end{array}$ & 0 & 0 & 0 & 0 & 0 \\
\hline $\begin{array}{l}\text { Got angry } \\
\text { when I } \\
\text { encouraged } \\
\text { them to eat } \\
\text { low-salt or } \\
\text { low-fat foods. }\end{array}$ & 0 & 0 & 0 & 0 & 0 \\
\hline $\begin{array}{l}\text { Told me } \\
\text { eating a low- } \\
\text { salt, low-fat } \\
\text { diet is a waste } \\
\text { of time. }\end{array}$ & 0 & 0 & 0 & 0 & 0 \\
\hline
\end{tabular}


The next section of questions are from the Perceived Stress Scale.

The reference for these questions is the following: Cohen, S., Kamarck, T., and Mermelstein, R. (1983). A global measure of perceived stress. Journal of Health and Social Behavior, 24, 386-396.

The questions in this scale ask you about your feelings and thoughts during the last month. In each case, you will be asked to indicate by selecting how often you felt or thought a certain way.

\begin{tabular}{|c|c|c|c|c|c|}
\hline & Never & $\begin{array}{l}\text { Almost } \\
\text { never }\end{array}$ & Sometimes & Fairly often & Very often \\
\hline $\begin{array}{l}\text { In the last } \\
\text { month, how } \\
\text { often have } \\
\text { you been } \\
\text { upset because } \\
\text { of something } \\
\text { that happened } \\
\text { unexpectedly? }\end{array}$ & $\mathrm{O}$ & 0 & $\mathrm{O}$ & $\mathrm{O}$ & $\mathrm{O}$ \\
\hline $\begin{array}{l}\text { In the last } \\
\text { month, how } \\
\text { often have } \\
\text { you felt that } \\
\text { you were } \\
\text { unable to } \\
\text { control the } \\
\text { important } \\
\text { things in your } \\
\text { life? }\end{array}$ & $\mathrm{O}$ & $\mathrm{O}$ & $\mathrm{O}$ & $\mathrm{O}$ & $\mathrm{O}$ \\
\hline $\begin{array}{l}\text { In the last } \\
\text { month, how } \\
\text { often have } \\
\text { you felt } \\
\text { nervous and } \\
\text { "stressed"? }\end{array}$ & 0 & 0 & $\mathrm{O}$ & $\mathrm{O}$ & $\mathrm{O}$ \\
\hline $\begin{array}{l}\text { In the last } \\
\text { month, how }\end{array}$ & O & O & O & O & O \\
\hline
\end{tabular}




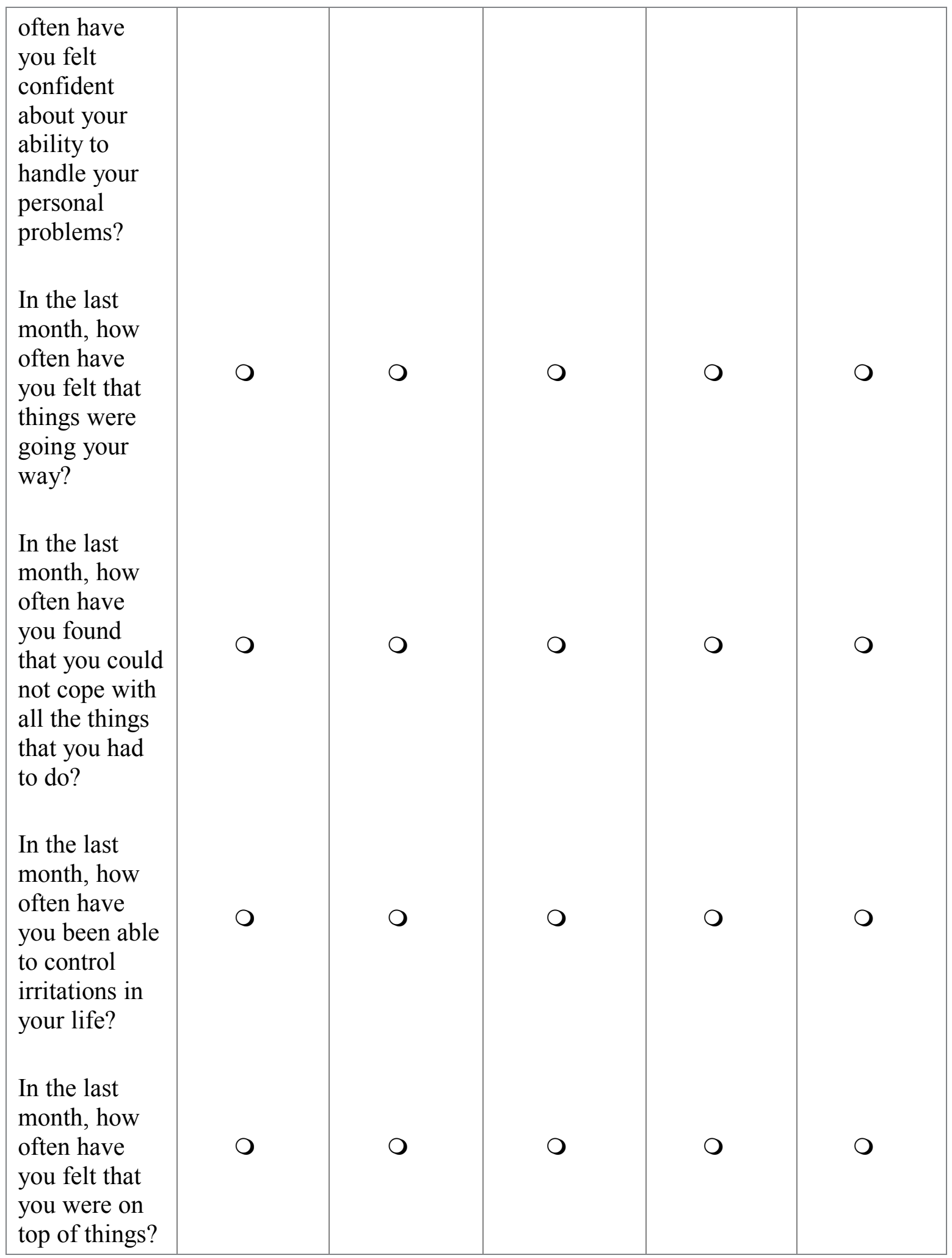




\begin{tabular}{|c|c|c|c|c|c|}
\hline $\begin{array}{l}\text { In the last } \\
\text { month, how } \\
\text { often have } \\
\text { you been } \\
\text { angered } \\
\text { because of } \\
\text { things that } \\
\text { were outside } \\
\text { of your } \\
\text { control? }\end{array}$ & 0 & 0 & 0 & 0 & 0 \\
\hline $\begin{array}{l}\text { In the last } \\
\text { month, how } \\
\text { often have } \\
\text { you felt } \\
\text { difficulties } \\
\text { were piling } \\
\text { up so high } \\
\text { that you could } \\
\text { not overcome } \\
\text { them? }\end{array}$ & 0 & 0 & 0 & 0 & 0 \\
\hline
\end{tabular}

How did you hear about this survey? (Check all that apply below) 


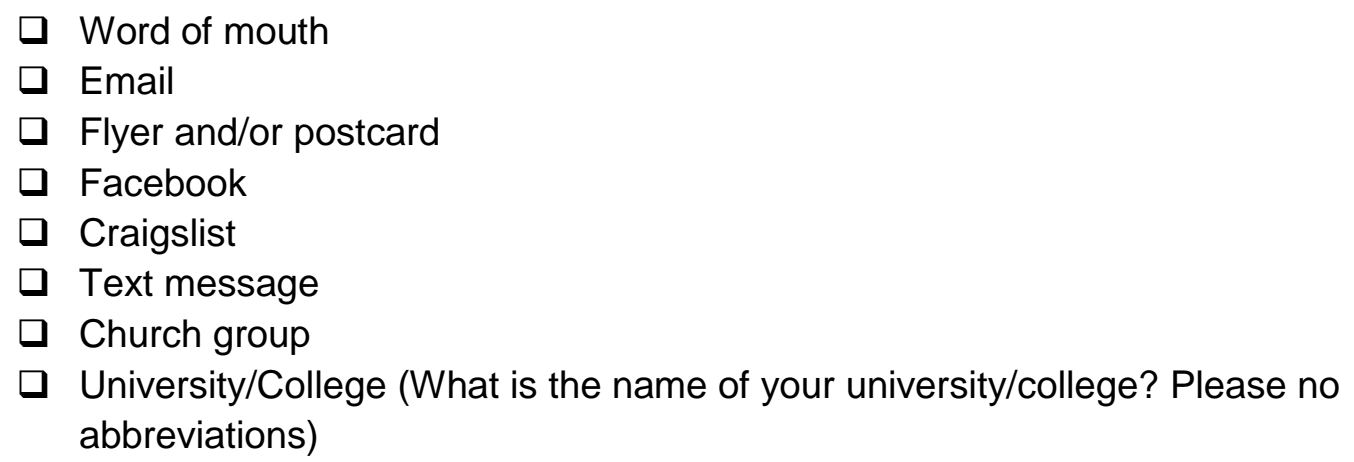

$\square$ Other (please specify)

Would you like to receive a list of mental health resources?

O Yes

O No

Would you like to receive the results of your dietary fat intake screening?

$\mathrm{O}$ Yes

O No

I am sorry, but you do not meet the criteria to participate in this online research study. You must live in the United States, read and speak English, self-identify as African American or Black, be 18 to 25 years old, and not be pregnant. However, if you have family and friends who meet the criteria, please share the web link with them to take the survey. Thank you for your interest. [Message participants received if they did not me survey criteria] 


\section{Appendix D Permission to Use Instruments}

From: Harold Koenig [koenig@geri.duke.edu]

Sent: Wednesday, February 20, 2013 12:17 PM

To: Shalonda Horton [shorton@mail.nur.utexas.edu]

Title: RE: Question regarding the Duke University Religion Index (DUREL)

Shalonda -- you have my permission -- see attached. HK

[2 attachments]

From: Shalonda Horton [shorton@mail.nur.utexas.edu]

Sent: Wednesday, February 20, 2013 11:57 AM

To: Harold Koenig [koenig@geri.duke.edu]

Title: Question regarding the Duke University Religion Index (DUREL)

Dear Dr. Koenig,

I hope this email finds you doing well.

I am interested in using your Duke University Religion Index (DUREL) scale for my dissertation, with your permission (Koenig, H. G. \& Büssing, A, 2010).

I would use your instrument in an on-line study with Black young adults, ages 18 to 25 years.

Thank you for your support of my development as a researcher.

Sincerely,

Shalonda

Shalonda Horton

MSN, BSN, RN

PhD Student

Assistant Instructor

The University of Texas at Austin

School of Nursing

512-659-5536 (cell)

512-475-8694 (office)

shorton@mail.nur.utexas.edu

From: Marie Lobo [mlobo@ salud.unm.edu]

Sent: Monday, February 11, 2013 12:37 PM 
To: Shalonda Horton [shorton@mail.nur.utexas.edu]

Title: RE: Question regarding your Economic Adequacy Scale

Shalonda, I am attaching a number of documents including the original scale. Please read the information attached to the EAS for original reliability information. Please acknowledge the source and you have permission to use.

[6 attachments]

Dr. Lobo

Marie L. Lobo, PhD, RN, FAAN

Professor

University of New Mexico

College of Nursing

MSC09 5350

1 University of New Mexico

Albuquerque, NM 87131-0001

505-272-2637

Fax 505-272-8901

e-mail:mlobo@salud.unm.edu

(UPS, FedEx, and Airborne Express Deliveries)

Nursing/Pharmacy Building

2502 Marble Ave., NE

Albuquerque, NM 87131-0001

This email and all files transmitted with it are confidential and intended solely for the use of the individual or entity to whom they are addressed. If you have received this email in error, please notify the sender by email and delete and destroy this message and its attachments. Thank you.

From: Shalonda Horton [shorton@mail.nur.utexas.edu]

Sent: Sunday, February 10, 2013 4:23 PM

To: Marie Lobo [mlobo@salud.unm.edu]

Title: Question regarding your Economic Adequacy Scale

Dear Dr. Lobo,

I am a PhD nursing student at the University of Texas at Austin. I recently read a couple of articles, which referred to your Economic Adequacy Scale.

I am interested in using your scale for my dissertation, with your permission. Is there documentation of the Cronbach's $\alpha$ coefficient for the instrument? And is it possible to have a copy of the original instrument? 
Thank you for your assistance and support of my development as a researcher.

Respectfully,

Shalonda

Shalonda Horton

MSN, BSN, RN

PhD Student

Assistant Instructor

The University of Texas at Austin

School of Nursing

512-659-5536 (cell)

512-475-8694 (office)

shorton@mail.nur.utexas.edu

From: Jim Sallis [jsallis@ucsd.edu]

Sent: Wednesday, February 20, 2013 4:02 PM

To: Shalonda Horton [shorton@mail.nur.utexas.edu]

Title: RE: Question regarding the Family and Friend Support for Healthy Eating Habits Scale

Hello Shalonda,

Thanks for your interest in these measures. You have my permission to use these scales or any others posted on my website.

We have used these measures with different numbers of items, different response scales, and modified wording. All these versions seem to be useful, so in my opinion you can adapt as needed, even though strictly speaking many people would say you need to use only the original version.

Scoring instructions are on the website. I don't have any other versions to share; you may have to retype the forms.

$\operatorname{Jim} S$

James F. Sallis, Ph.D.

Distinguished Professor of Family and Preventive Medicine

Chief, Division of Behavioral Medicine

University of California, San Diego, mail code 0824

Director, Active Living Research 
3900 Fifth Avenue, Suite 310

San Diego, CA 92103

ph: 619-260-5535; fax 619-260-1510

Email: jsallis@ucsd.edu; Website: http://sallis.ucsd.edu

From: Shalonda Horton [shorton@mail.nur.utexas.edu]

Sent: Wednesday, February 20, 2013 4:02 PM

To: Jim Sallis [jsallis@ ucsd.edu]

Title: Question regarding the Family and Friend Support for Healthy Eating Habits Scale

Dear Dr. Sallis:

I do apologize for the additional email, but I also read your article about the Family and Friend Support for Heart Healthy Eating Habits Scale (1987).

I am interested in using both scales for my dissertation, with your permission. My study is designed to be conducted on-line and I wanted to upload the questions from your original scales. I saw that there is a version on your website (8-point Likert scale), but I would like to use the original version for my study (5-point Likert scale).

May I please have a copy of the scales, along with the scoring instructions, to use for my dissertation?

Again, thank you in advance for your help.

Respectfully, Shalonda

Shalonda Horton MSN, BSN, RN

PhD Student Assistant Instructor

The University of Texas at Austin

School of Nursing

512-659-5536 (cell)

512-475-8694 (office)

shorton@mail.nur.utexas.edu 
From: Sheldon Cohen's website Laboratory for the Study of Stress, Immunity and Disease http://www.psy.cmu.edu/ scohen/

\section{Dr. Cohen's Scales:}

We welcome copies (e-mail is $\mathrm{OK}$ ) of any in press or published papers using any of Dr. Cohen's scales that you are willing to share with us, and thank you in advance for your generosity. They will not be redistributed or linked without your permission.

Permissions: Permission for use of scales is not necessary when use is for nonprofit academic research or nonprofit educational purposes. For other uses, please contact Ellen Conser at conser@andrew.cmu.edu for instructions. 


\section{NutritionQuest \\ 15 Shattuck Square, Suite 288, Berkeley, CA 94704-1151 \\ Phone 510-704-8514 / Fax 510-704-8996 \\ www.NutritionQuest.com \\ Licensing Agreement for Use of "Block Fat Screener" (C) \\ For study: Factors Influencing Dietary Fat Intake \\ Among Black Young Adults}

This agreement, effective the $1^{\text {st }}$ day of July, 2013, is between NutritionQuest, located at 15 Shattuck Square, Suite 288 Berkeley, CA 94704-1151, and Shalonda Horton (hereafter referred to as Licensee), located at 1710 Red River, Austin, TX 78701, who agrees to the following terms and conditions.

1. LICENSE.

a. Licensee has a nonexclusive right to use the "Block Fat Screener" (C) (hereafter referred to as SCREENER), and the accompanying analysis algorithms (hereafter referred to as ALGORITHMS) for use in study entitled "Factors Influencing Dietary Fat Intake Among Black Young Adults.

b. SCREENER will be administered to 25 respondents, one time, over the course of six weeks, for a total of 25 respondent-administrations.

a. At the conclusion of this 6 week study, the SCREENER and ALGORITHMS will be removed from any computers on which they were installed.

2. COPYRIGHT AND CONFIDENTIALITY.

a. Licensee agrees that NutritionQuest will provide to Licensee under this agreement SCREENER and ALGORITHMS containing information that is confidential and proprietary in nature, and which are protected by copyrights held by NutritionQuest.

b. Licensee will include the following notice on all pages of SCREENER and ALGORITHMS: "C NutritionQuest, Berkeley, CA (510) 704-8514"

c. NutritionQuest retains title to the SCREENER AND ALGORITHMS.

d. Licensee agrees to use reasonable efforts to protect the SCREENER and ALGORITHMS from unauthorized use, reproduction, distribution or publication.

3. USES NOT PERMITTED.

a. Licensee may not rent, lease, lend or sublicense, or transfer the SCREENER or ALGORITHMS without prior approval.

b. Licensee may not publish, sell or distribute components of the SCREENER or ALGORITHMS.

c. Licensee MAY NOT USE THE SCREENER OR ALGORITHMS FOR COMMERCIAL PURPOSES OR FEE FOR SERVICE.

d. Licensee may not decompile, disassemble or reverse engineer the SCREENER OR ALGORITHMS.

e. Licensee may not use the ALGORITHMS to create or expand other nutrient databases.

f. This license is with Shalonda Horton only. Licensee may not use the SCREENER or ALGORITHMS to process data generated by researchers outside of Licensee's institution. 
g. Licensee may not remove or obscure any copyright, confidential or trademark notices.

h. Licensee may not use the SCREENER or ALGORITHMS or any part thereof in the development or operation of a web site accessible to the general public.

4. DEFINITION. "Derivative Work" shall mean a work that is based upon one or more pre-existing works, such as a revision, modification, translation, abridgement, condensation, expansion, or any other form in which such pre-existing works may be recast, transformed, or adapted, and that, if prepared without authorization of the owner of the copyright in such pre-existing work, would constitute a copyright infringement. For purposes hereof, a Derivative Work shall also include any compilation that incorporates such a pre-existing work.

5. MODIFICATIONS.

a. Licensee may not modify the SCREENER OR ALGORITHMS without the express written permission of NutritionQuest.

b. Any derivative works (as defined in Section 4) developed by the Licensee under this agreement are the property of NutritionQuest and subject to the copyrights held by NutritionQuest (see Section 2).

6. DURATION. If Licensee breaches this license, NutritionQuest can terminate the license upon notifying Licensee in writing. NutritionQuest can also enforce its other legal rights.

7. WARRANTY. NutritionQuest warrants that the electronic files on which the SCREENER AND ALGORITHMS are provided are free from defects in workmanship or material under normal use for 90 days from the date of shipment.

8. WARRANTY LIMITATION. The SCREENER and ALGORITHMS are provided "as is." Except for the express warranty above, NutritionQuest grants no other warranties, either express or implied, including all implied warranties of merchantability and fitness for a particular purpose.

9. LIMITATION OF LIABILITY. NutritionQuest will not be liable for any indirect, consequential, or incidental damages arising out of the use of the SCREENER OR ALGORITHMS, even if NutritionQuest has been advised of the possibility of such damages or claim.

10. FEES. Licensee will pay NutritionQuest $\$ 0.75$ for each respondent-administration. The total amount due under this contract is $\$ 18.75$, which is determined as follows: $\$ 0.75 \times 25$ respondentadministrations (as indicated in 1.b. above). All fees are due upon signing of this agreement.

\section{GENERAL.}

a. Licensee acknowledges that Licensee has read and understands every provision of this license and that Licensee agrees to be bound by its terms and conditions.

b. This license represents the entire agreement and understanding of the parties regarding the SCREENER AND ALGORITHMS and this license supersedes any prior purchase order, communication, advertising, or representation. 
Licensing Agreement for Use of "Block Fat Screener" (C) For study: Factors Influencing Dietary Fat Intake Among Black Young Adults

\author{
LICENSEE: \\ Shalonda Horton, MSN, BSN, RN \\ PhD Student at \\ The University of Texas at Austin, School of Nursing \\ Authorized representative: \\ Name: Shalonda Horton \\ Title: PhD student \\ Signature: Shalonda Horton \\ Date:

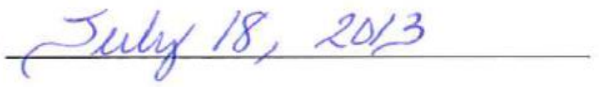 \\ Address: \\ Shalonda Horton \\ 1710 Red River, \\ Austin, TX 78701 \\ Phone: (512) 659-5536 cell \\ Fax: (512) 471-3688 \\ Email: shorton@mail.nur.utexas.edu
}




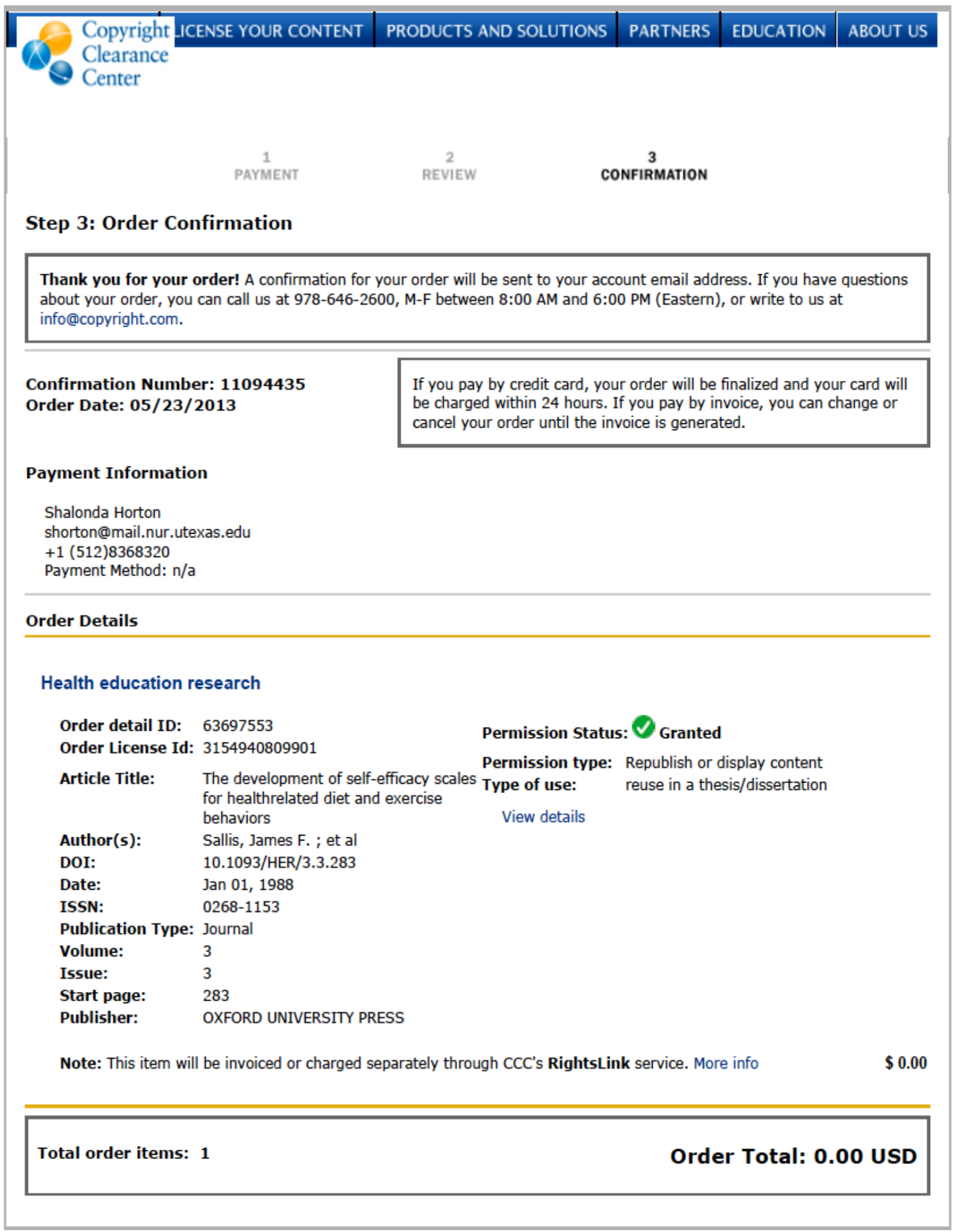


Permission to use the Motivators of and Barriers to Health-Smart Behaviors Inventory

The Licensee has read the MB-HSBI ${ }^{\mathrm{TM}}$ Binding Product License Agreement and accepts and agrees to the terms outlined in the Agreement. M \& B accepts this Agreement, the MB-HSBI ${ }^{T M}$ Product Order Form, and payment to cover total fees, and grants a License of use to the purchaser subject to the terms of this Agreement.

\section{AGREED TO BY:}

Shalonda Horto

Licensee Name
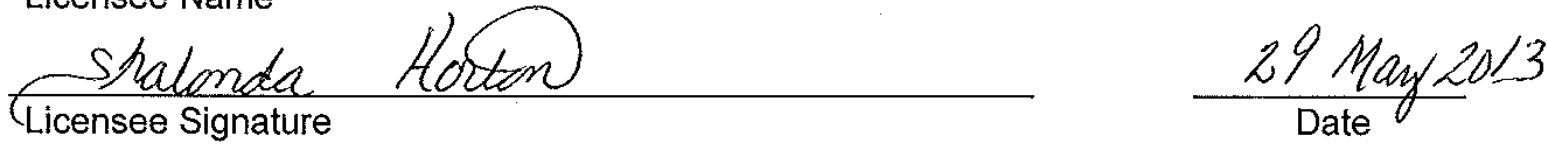

Purchaser's Name (If different from the Licensee)

Purchaser's Signature

Date 


\section{Appendix E Flyer}

\section{Are you between the ages of 18-and 25 ?}

\section{Then you may be eligible to participate in an online research study.}

Purpose of this study: To examine factors that may influence dietary fat intake.

It takes about 15 minutes to complete the survey.

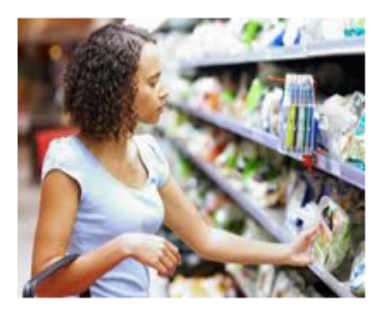

To take the online survey, click on the below web link:

Web link to survey INSERT LINK HERE

After completing the online survey, you can enter drawings for a chance to receive an electronic tablet and one of eleven $\$ 10$ value gift cards.
For more information, contact:

Shalonda Horton

MSN, RN

PhD Student

University of Texas at Austin School of Nursing

dietaryfatresearchstudy@gmail.com

(512) 475-8694 office

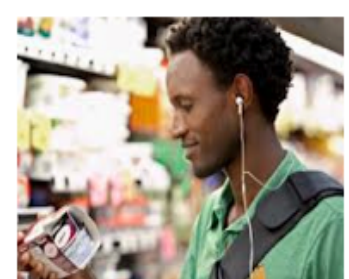




\section{Appendix F Postcard}

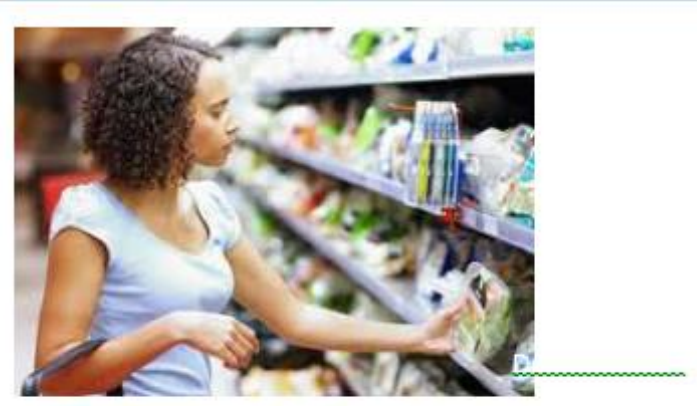

Are you between the ages of 18-and 25?

Then you may be eligible to participate in a study about factors influencing dietary fat intake.

A...
Purpose of this study:

To examine factors that may influence dietary fat intake.

If you agree to participate:

You will be asked to answer questions honestly in an

anonymous online survey.

Estimated time to participate:

The one time online survey takes about 15 minutes to complete.

Incentives:

After completing the online survey you will be entered into drawings for an electronic tablet and one of eleven $\$ 10$ gift cards.

The information you share may help to develop health promotion programs.

A...

\section{Black young adults \& dietary fat intake}

Contact:
Shalonda Horton
MSN, BSN, RN
PhD Student
University of Texas at Austin
School of Nursing
dietarvfatresearchstudv@gmail.com
(512) $475-8694$ office

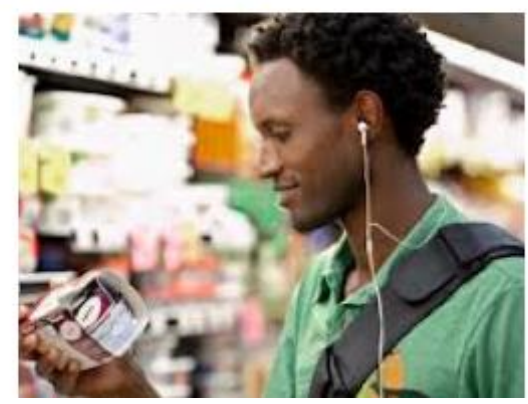




\section{Appendix G Email Message to Organizations/Businesses}

Date

Dear

Shalonda Horton, from The University of Texas at Austin (School of Nursing), recently spoke to our group about participating in a research study.

The purpose of the study is to examine factors that may influence dietary fat intake. The study involves completing an anonymous online survey, which takes about 15 minutes to complete. The questions consist of items such as, but not limited to, the following:

- Demographic information: age, marital status, and highest education level completed

- Cultural information: religion and spirituality

- Barriers related to eating healthy

- Social support in eating healthy

- Stress and stress related to racism

- Dietary fat intake

To participate in the study participants need to: live in the United States, self-identify as African American or Black, be 18 to 25 years old, and if female, not be pregnant.

After completing the online survey, participants can enter drawings for a chance to receive an electronic tablet and one of eleven $\$ 10$ value gift cards.

I have also included the web link to the online survey and a flyer to share with your (name of organization/business) members/patrons: (insert link to survey).

Thank you in advance for helping me to examine factors that may influence dietary fat intake.

Sincerely,

Shalonda Horton

M.S.N., B.S.N., R.N.

The University of Texas at Austin

School of Nursing

shorton@mail.nur.utexas.edu

512-475-8694 


\section{Appendix H Email Message for Fat Screening Results}

Thank you for participating in my research study that examines factors influencing fat intake among Black young adults.

Your Dietary Fat Screener Score was [insert score]. This score means that your diet is [low, average, high, very high] in fat, probably [insert percent $\%$ ] of your calories come from fat. The American Heart Association recommends limiting your total fat intake to $25-35 \%$.

Below you will find a list of Healthy Eating Resources:

Dietary fat: http://www.nlm.nih.gov/medlineplus/dietaryfats.html

Dietary fat: http://www.cdc.gov/nutrition/everyone/basics/fat/index.html

Healthy eating for a healthy weight:

http://www.cdc.gov/healthyweight/healthy_eating/index.html

Planning meals: http://www.cdc.gov/healthyweight/healthy eating/meals.html

Cutting calories: http://www.cdc.gov/healthyweight/healthy_eating/cutting_calories.html

Healthy recipes: http://www.cdc.gov/healthyweight/healthy_eating/recipes.html

ChooseMyPlate.gov: http://www.choosemyplate.gov

Please share the survey with your young adult family members/friends: [insert web link]

Again, thank you for your feedback in improving the online survey.

If you have any questions, please contact me by telephone or email: (512) 475-8694, dietaryfatresearchstudy@gmail.com.

Respectfully,

Shalonda Horton

Shalonda Horton

MSN, RN, PhD Student

The University of Texas at Austin, School of Nursing

dietaryfatresearchstudy@gmail.com

(512) 475-8694 office number 


\section{Appendix I Email Message for Mental Health Resources}

Thank you for participating in my research study that examines factors influencing fat intake among Black young adults.

Per your request, you will find a list of Mental Health Resources below.

Mental health: http://www.nlm.nih.gov/medlineplus/mentalhealth.html

General resources: http://www.cdc.gov/mentalhealth/gen-resources.htm

Hogg Foundation for Mental Health: http://www.hogg.utexas.edu

Travis County Mental Health Services:

http://www.caction.org/health/PrescriptionForWellness/Appendices/AppendixC.htm

National Suicide Prevention Lifeline: http://www.suicidepreventionlifeline.org

Texas Suicide Prevention: http://www.texassuicideprevention.org

If you have any questions, please contact me by telephone or email: (512) 475-8694, dietaryfatresearchstudy@gmail.com.

Respectfully,

Shalonda Horton

Shalonda Horton

MSN, RN, PhD Student

The University of Texas at Austin, School of Nursing

dietaryfatresearchstudy@gmail.com

(512) 475-8694 office number 


\section{Appendix J Email Reminder}

Date

Dear

On [date] you started an online survey titled Factors Influencing Dietary Fat Intake Among Black Emerging Adults. I have not yet received your completed online survey at this time. I hope you get an opportunity to complete the survey soon.

You can click on your saved link to complete the survey.

If you need to start a new survey, the web link to the survey is: (insert link to survey).

If you are having difficulty opening the web link, please let me know so we can fix it. If you do not have access to a computer and Internet, contact your local library to use their computers for free to complete the online survey.

If you have any questions, please contact me by telephone or email: (512) 475-8694, dietaryfatresearchstudy@gmail.com.

Respectfully,

Shalonda Horton

Shalonda Horton

MSN, RN, PhD Student

The University of Texas at Austin, School of Nursing

dietaryfatresearchstudy@gmail.com

(512) 475-8694 office number 


\section{Appendix K Consent to Participate in Research (paper survey)}

\section{Identification of Investigator and Purpose of Study}

You are invited to participate in a research study, entitled "Factors Influencing Dietary Fat Intake Among Black Young Adults." The study is being conducted by Shalonda Horton in the School of Nursing of The University of Texas at Austin, 1710 Red River, Austin, Texas 78701, 512-4758694 (office number), dietaryfatresearchstudy@gmail.com.

The purpose of this online research study is to examine factors that may influence dietary fat intake. Your participation in the study will contribute to a better understanding of what influences dietary fat intake, an important risk factor for heart disease and weight gain. You are free to contact the investigator at the above address, phone number, and/or email address to discuss the study. In order to participate in this study, you must be 18 to 25 years old, self-identify as Black, and speak and read English. You will be excluded if you are pregnant, due to your special nutritional requirements, or live outside of the U.S.

If you agree to participate:

- You, and not someone else (e.g., friend or family member), agree to complete a survey

- You agree to complete the survey only once and not multiple times (one completed survey per person)

- The confidential survey will take approximately 15 to 25 minutes of your time to complete.

- You will complete a survey with questions that assess the following information

$\circ$ Demographic information such as your age, marital status, and highest education level completed

- Cultural information such as your level of spirituality

- Barriers related to eating healthy

- Social support in eating healthy

- Stress

$\ominus$ Dietary fat intake-

- This survey will be open until 220 participants have completed the study. To thank you for your time in completing the survey, you will be given a $\$ 1.29$ value gift card to Amazon.com to use for a music download. The $\$ 1.29$ value gift card will be given to you in person upon completion of the survey.

\section{Risks/Benefits/Confidentiality of Data}

There are no known risks to participating in this study. If the survey questions make you feel uncomfortable, you may stop participating in the study, and a list of mental health resources is available upon request at the end of the survey. There are no benefits to participating in this study. At your request, your dietary fat screening intake results will be given to you in person upon completion of the survey. There will be no costs for participating in this study. A limited number of research team members will have access to the data during data collection and analysis. 


\section{Participation or Withdrawal}

Your participation in this study is voluntary. You may decline to answer any question and you have the right to withdraw from participation at any time. Withdrawal will not affect your relationship with The University of Texas in anyway. If you do not want to participate either simply tell the investigator that you want to stop and take your survey with you to destroy.

\section{Contacts}

If you have any questions about the study, contact one of the following researchers: Shalonda Horton, MSN, RN, at (512) 475-8694 (office number) or send an email to dietaryfatresearchstudy@gmail.com or Gayle Timmerman, PhD, RN, at (512) 471-9087 (office number) and/or gtimmerman@mail.utexas.edu. This study has been reviewed by The University of Texas at Austin Institutional Review Board and the study number is [STUDY NUMBER].

\section{Questions about your rights as a research participant.}

If you have questions about your rights or are dissatisfied at any time with any part of this study, you can contact, anonymously if you wish, the Institutional Review Board by phone at (512) 471-8871 or email at orsc@uts.cc.utexas.edu.

If you agree to participate in this research study, you will give consent by telling the investigator that you want to take the survey. The investigator will then give you a survey to complete and you will follow the instructions provided in the survey.

Thank you.

Keep this copy of the document (consent) for your records. 


\section{Appendix L Healthy Eating Resources (paper survey)}

Thank you for participating in my research study that examines factors influencing fat intake among Black young adults.

Your Dietary Fat Screener Score was [insert score].

Scoring Key: If your score is

0-7: This score means that your diet is very low in fat, probably less than $25 \%$ of your calories come from fat. You have a healthy fat screener score, congratulations! The American Heart Association recommends limiting your total fat intake to 25-35\%.

8-14: This score means that your dietary fat intake is about average, probably between $30-35 \%$ of your calories come from fat. The American Heart Association recommends limiting your total fat intake to no more than $25-35 \%$.

15-22: This score means your diet is high in fat, probably higher than $35 \%$ of your calories come from fat. The American Heart Association recommends limiting your total fat intake to no more than $25-35 \%$.

$23+$ : This score means that your diet is very high in fat, probably $40-50 \%$ of your total calories come from fat. The American Heart Association recommends limiting your total fat intake to $25-35 \%$.

Below you will find a list of Healthy Eating Resources:

Dietary fat: http://www.nlm.nih.gov/medlineplus/dietaryfats.html

Dietary fat: http://www.cdc.gov/nutrition/everyone/basics/fat/index.html

Healthy eating for a healthy weight:

http://www.cdc.gov/healthyweight/healthy_eating/index.html

Planning meals: http://www.cdc.gov/healthyweight/healthy_eating/meals.html

Cutting calories: http://www.cdc.gov/healthyweight/healthy_eating/cutting_calories.html

Healthy recipes: http://www.cdc.gov/healthyweight/healthy_eating/recipes.html

ChooseMyPlate.gov: http://www.choosemyplate.gov 
Please share the survey with your young adult family members/friends: [insert web link] Again, thank you for your feedback in improving the online survey.

If you have any questions, please contact me by telephone or email: (512) 475-8694, dietaryfatresearchstudy@gmail.com.

Respectfully,

Shalonda Horton

MSN, RN, PhD Student

The University of Texas at Austin, School of Nursing

dietaryfatresearchstudy@gmail.com

(512) 475-8694 office number 


\section{Appendix M Histograms}

Gender outliers included

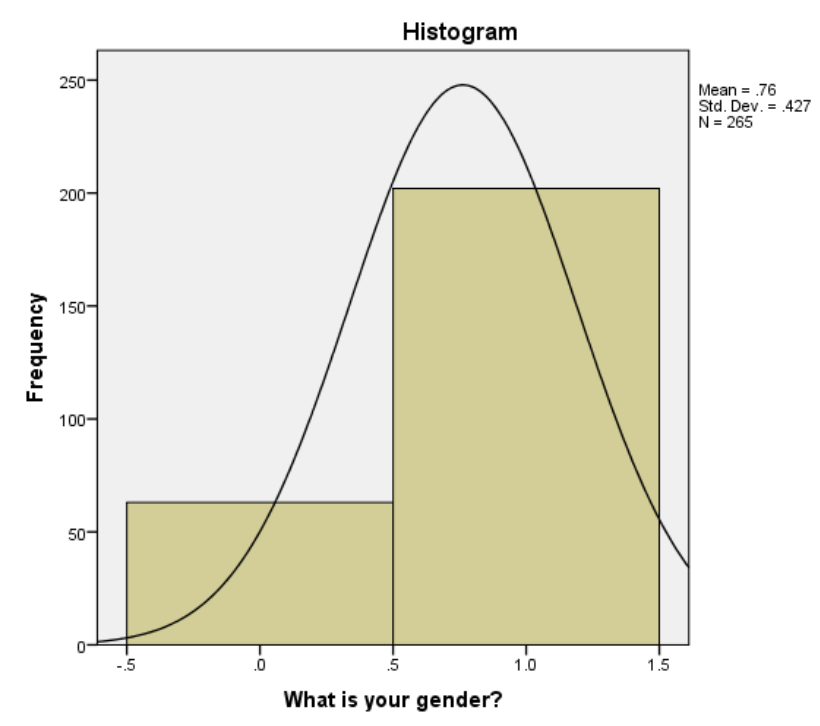

Gender outliers not included

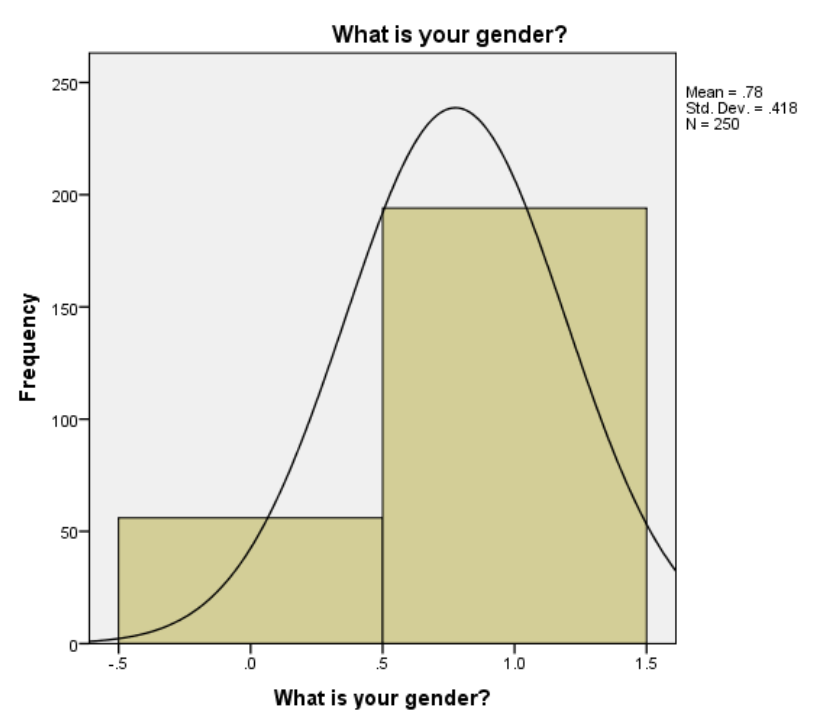


BMI outliers included

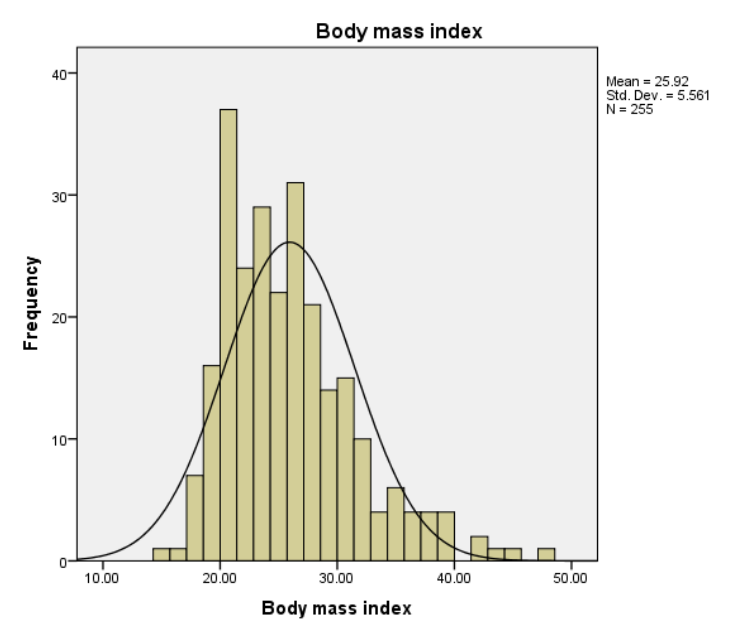

BMI outliers not included

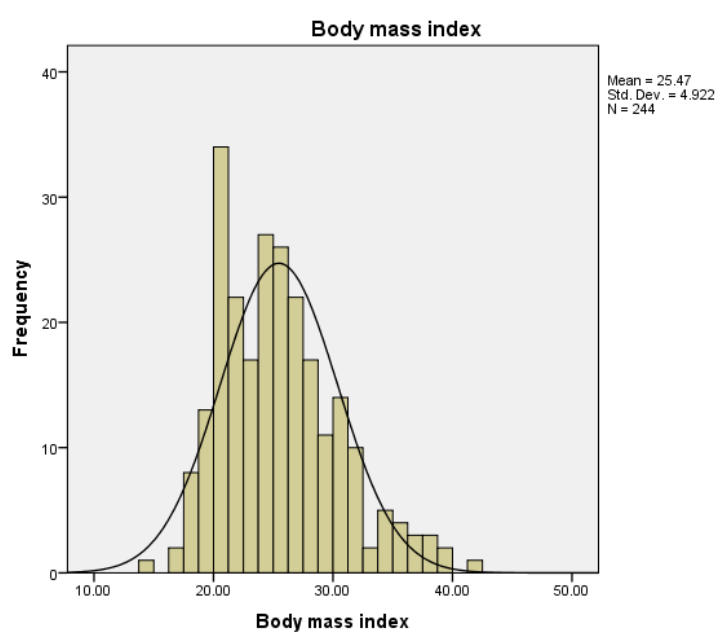

BMI (square root transformation) outliers not included

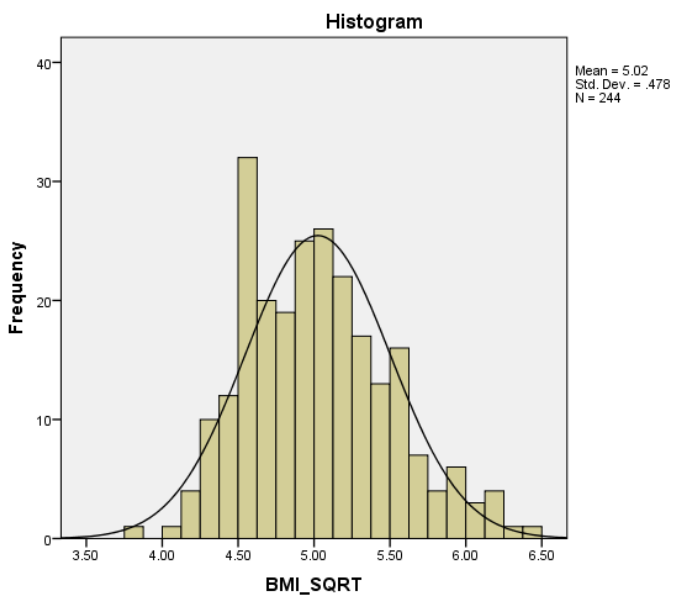

291 
DFI outliers included

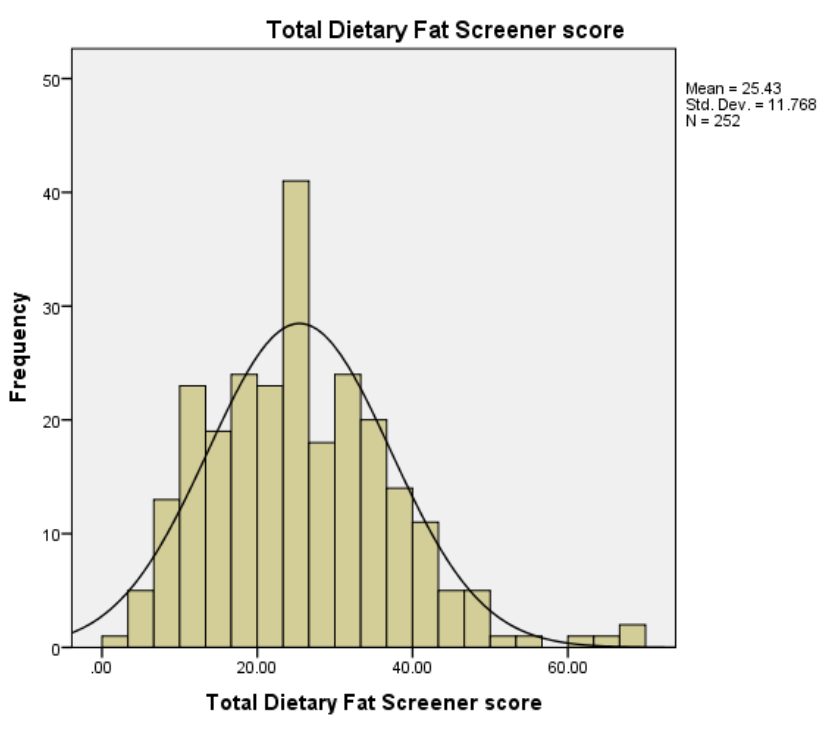

DFI outliers not included

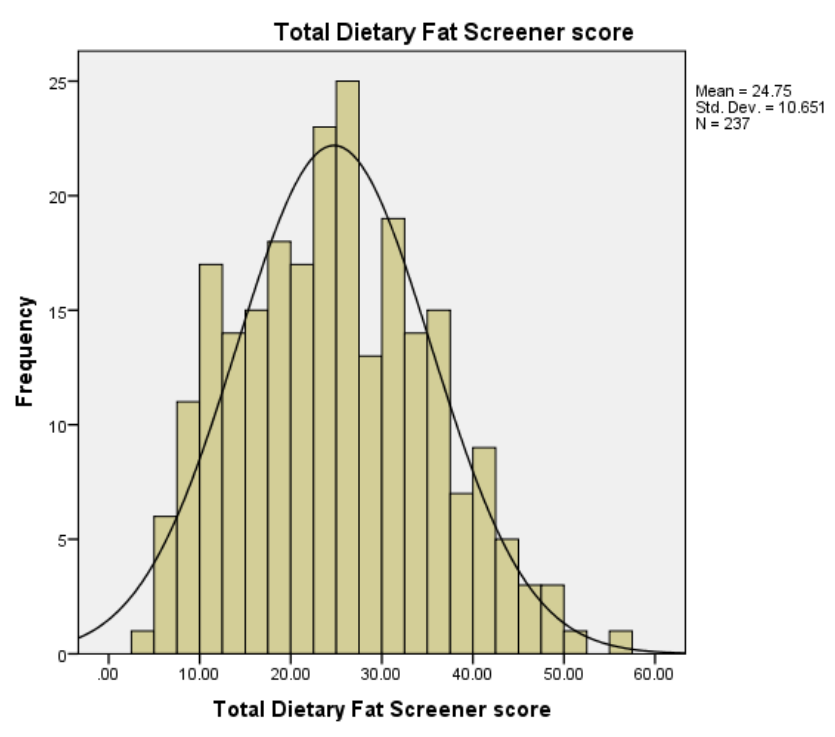


Intrinsic Religiosity Subscale outliers included

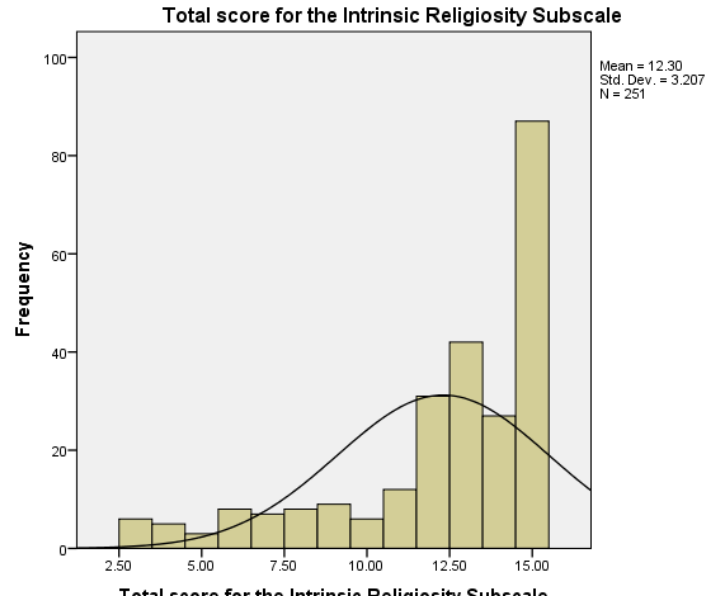

Intrinsic Religiosity Subscale outliers not included

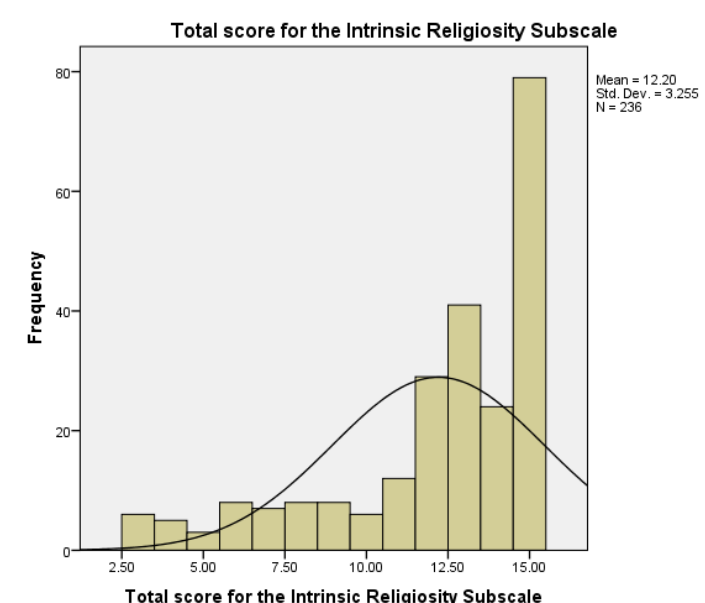

Intrinsic Religiosity Subscale (reflect/logarithm transformation) outliers not included

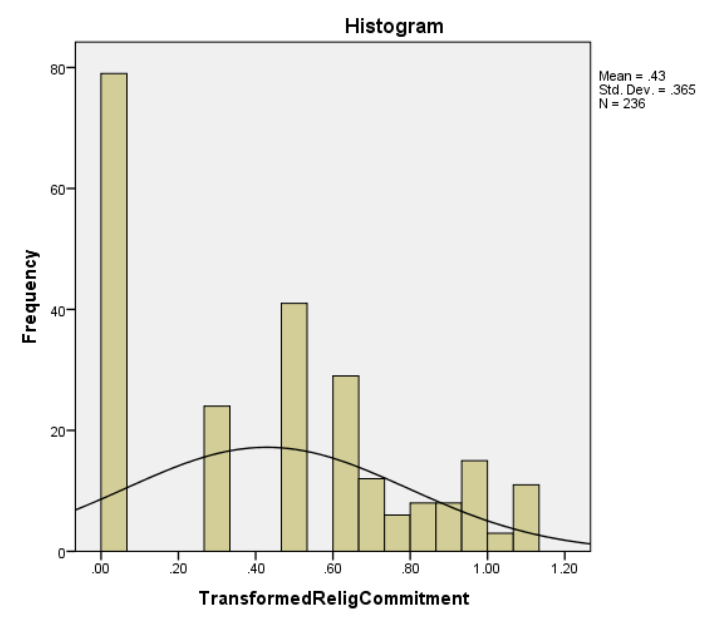

293 
Income Adequacy Scale outliers included Total score for the Income Adequacy Scal

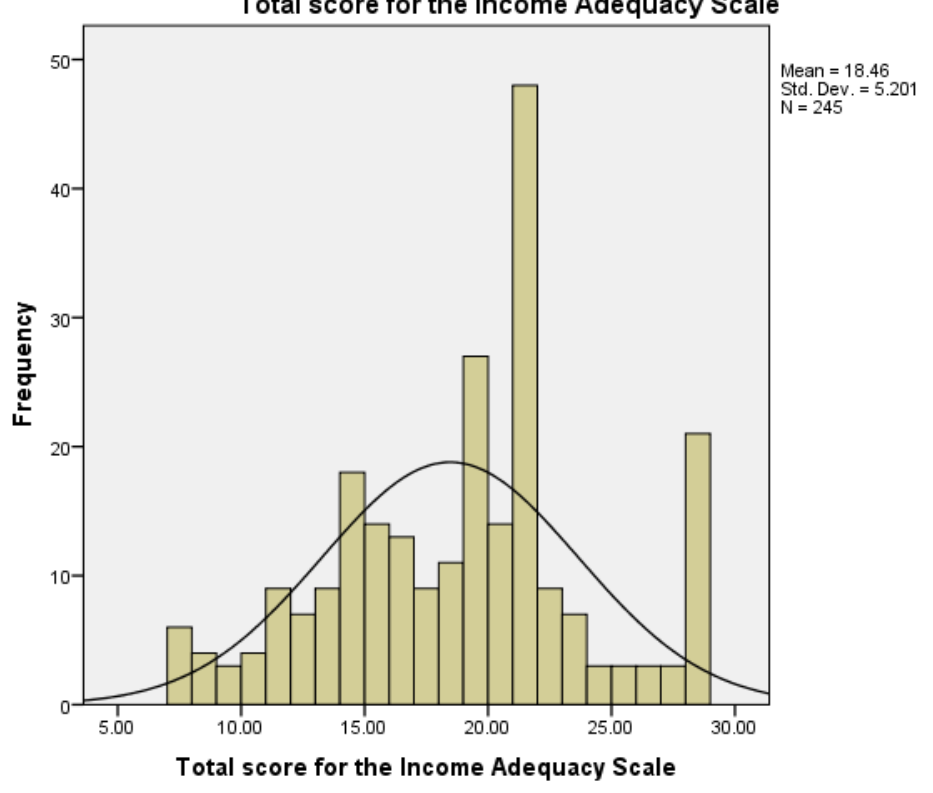

Income Adequacy Scale outliers not included

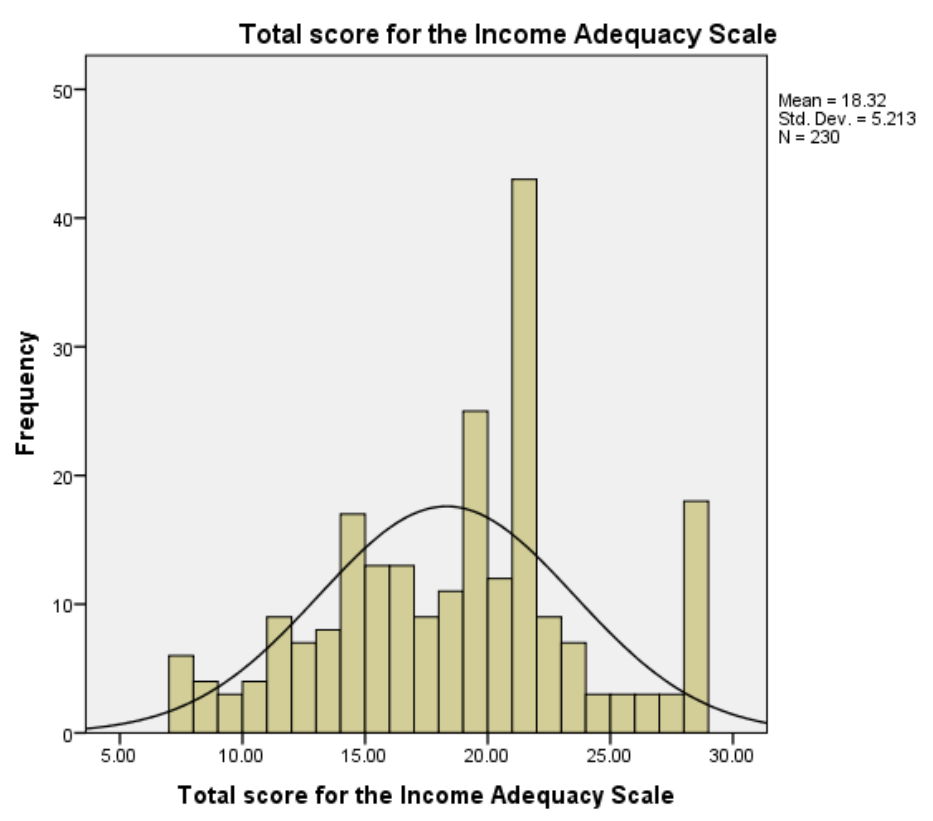


Barriers Subscale outliers included

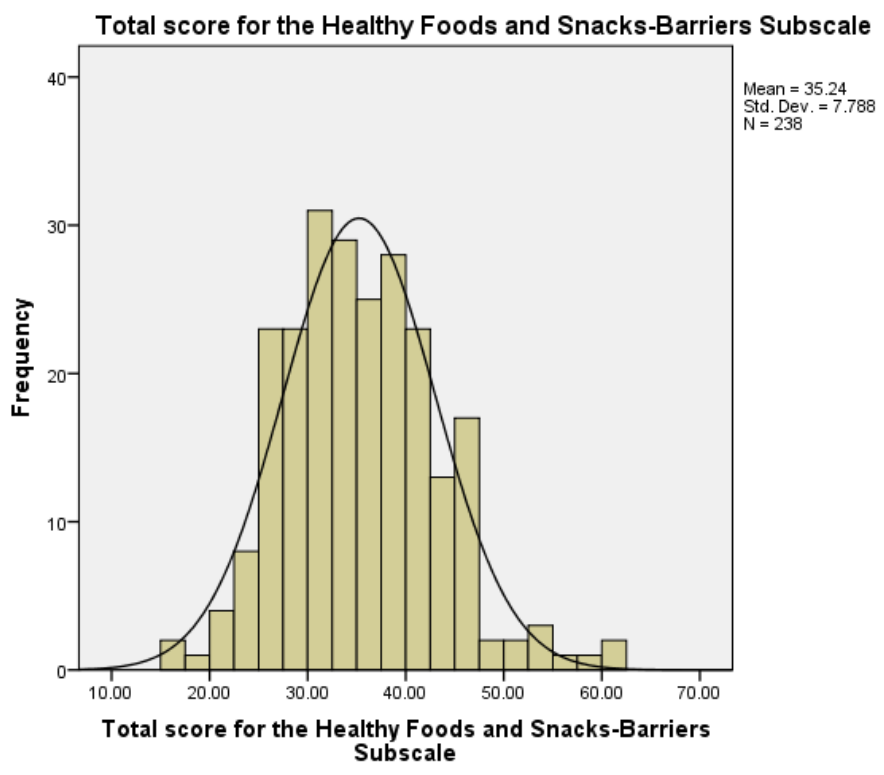

Barriers Subscale outliers not included

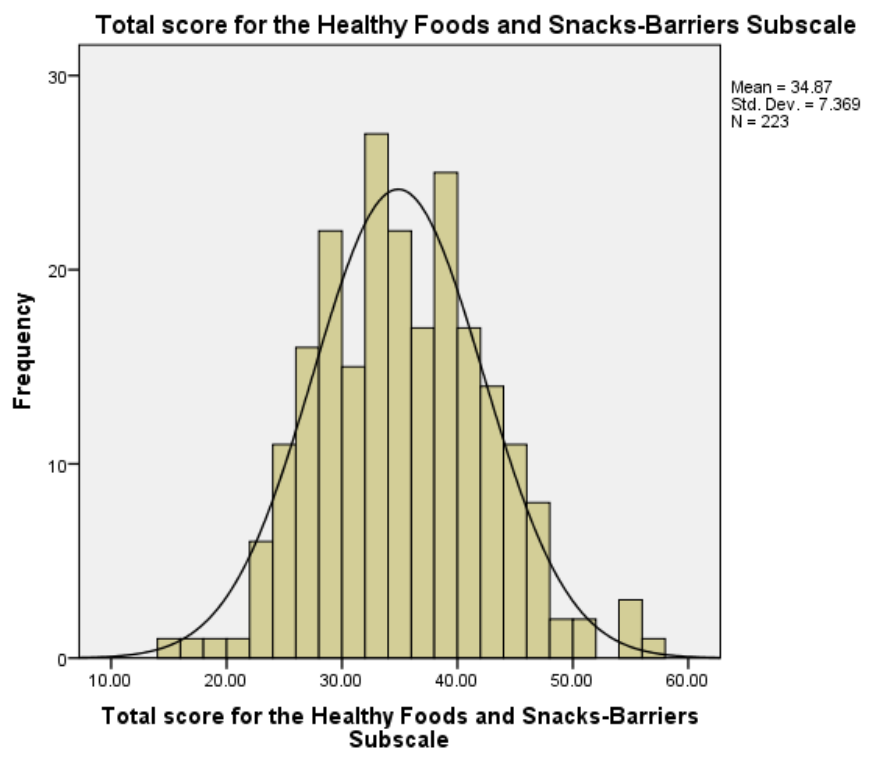


Resisting Relapse Subscale outliers included

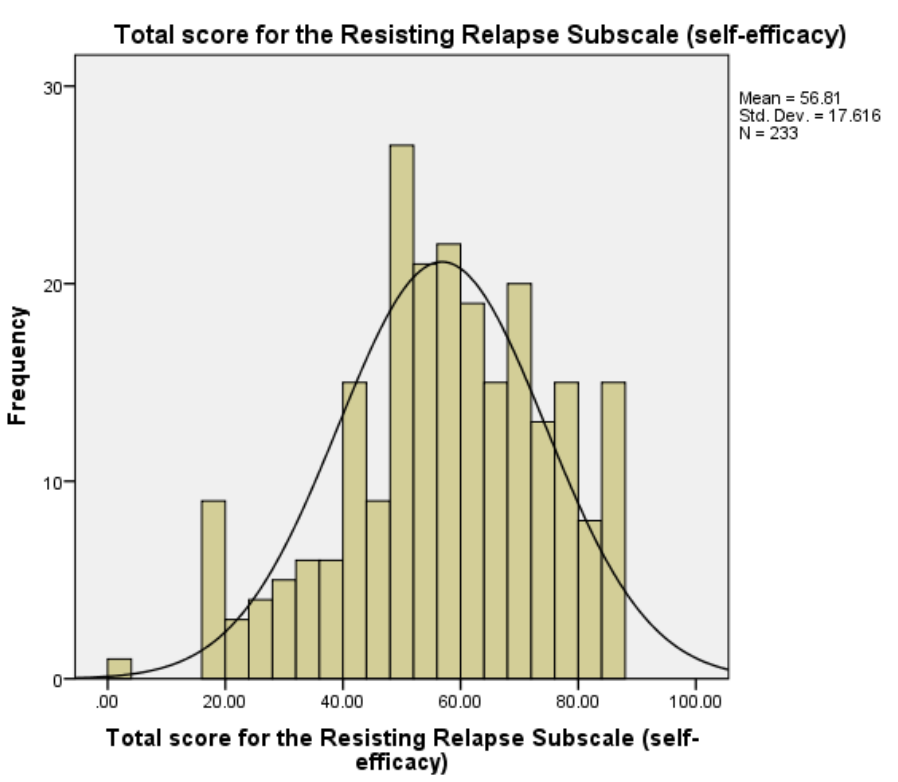

Resisting Relapse Subscale outliers not included

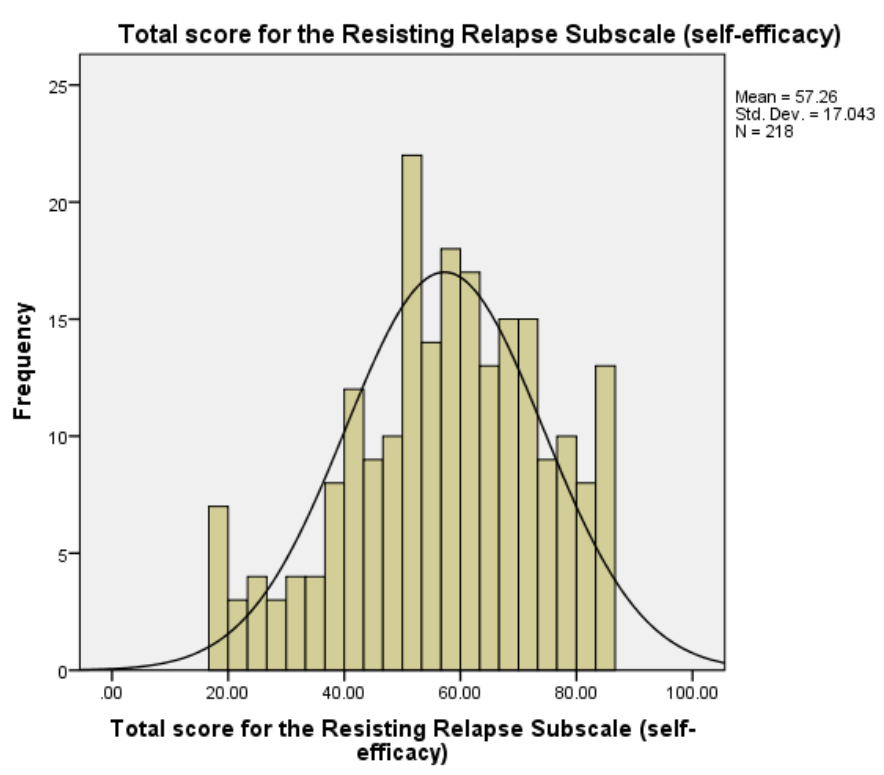


Family Support Scale outliers included

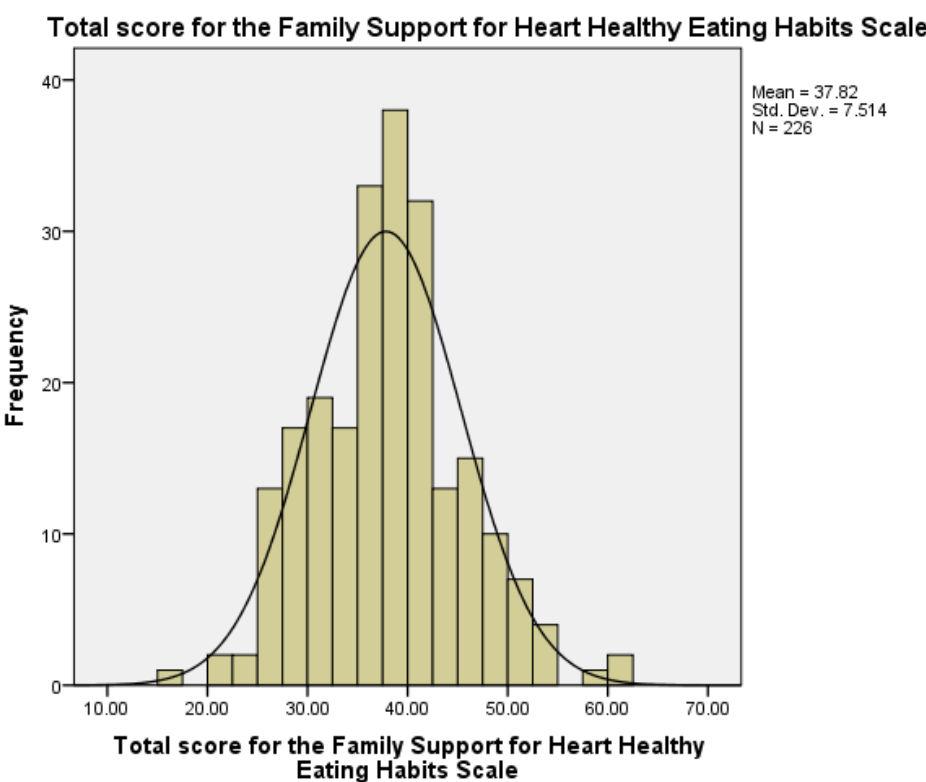

Family Support Scale outliers not included

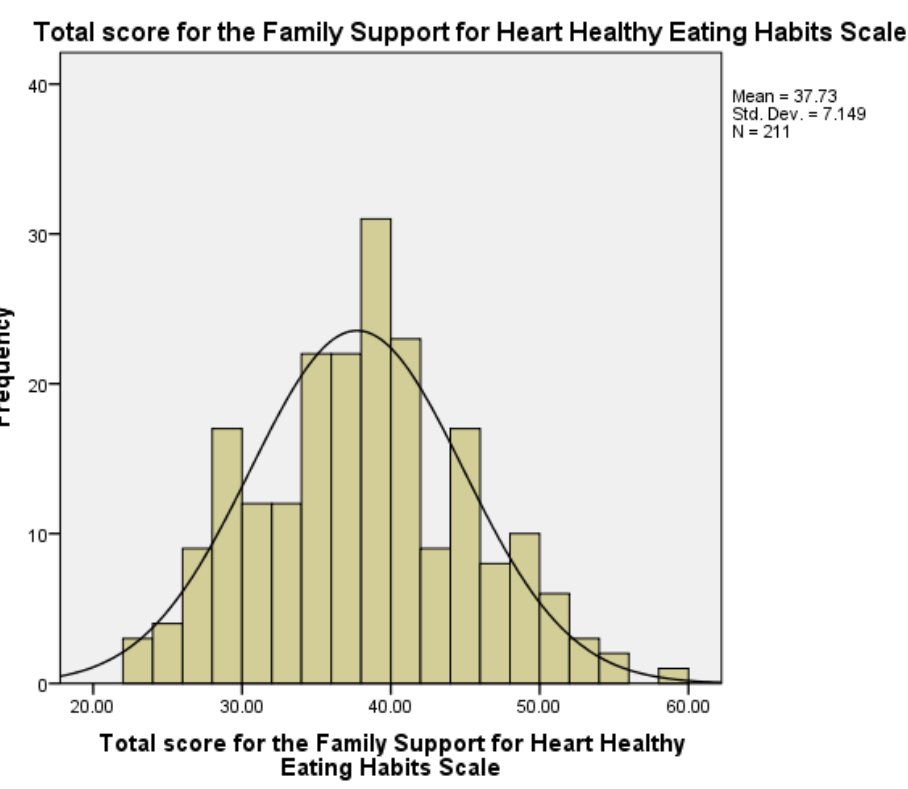


Friend Support Scale outliers included

Total score for the Friend Support for Heart Healthy Eating Habits Scale

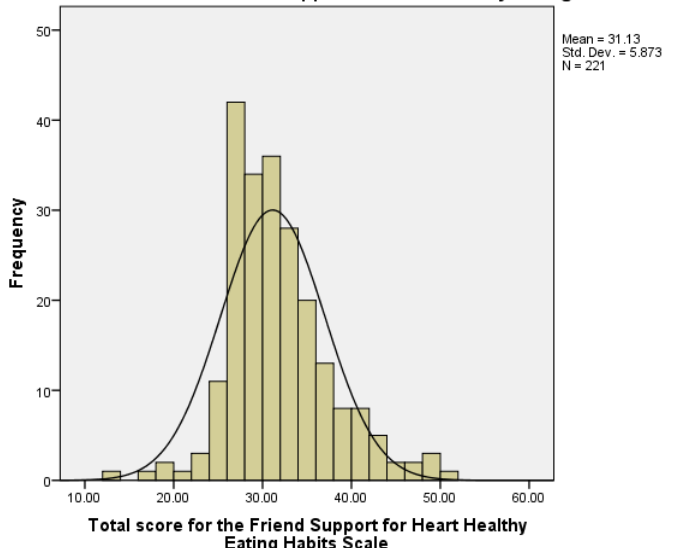

Friend Support Scale outliers not included Total score for the Friend Support for Heart Healthy Eating Habits Scale

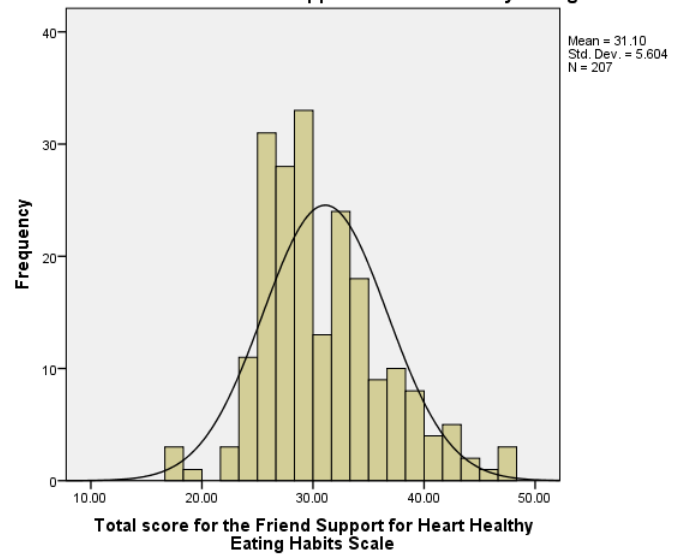

Friend Support Scale (square root transformation) outliers not included

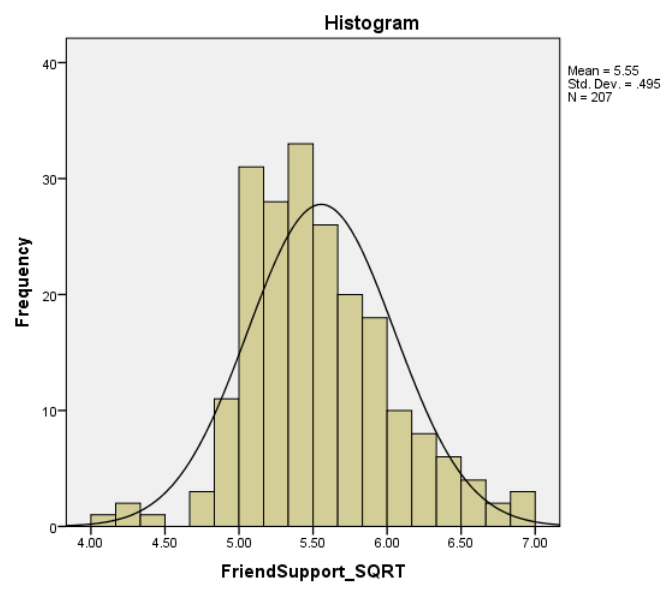


Perceived Stress Scale outliers included

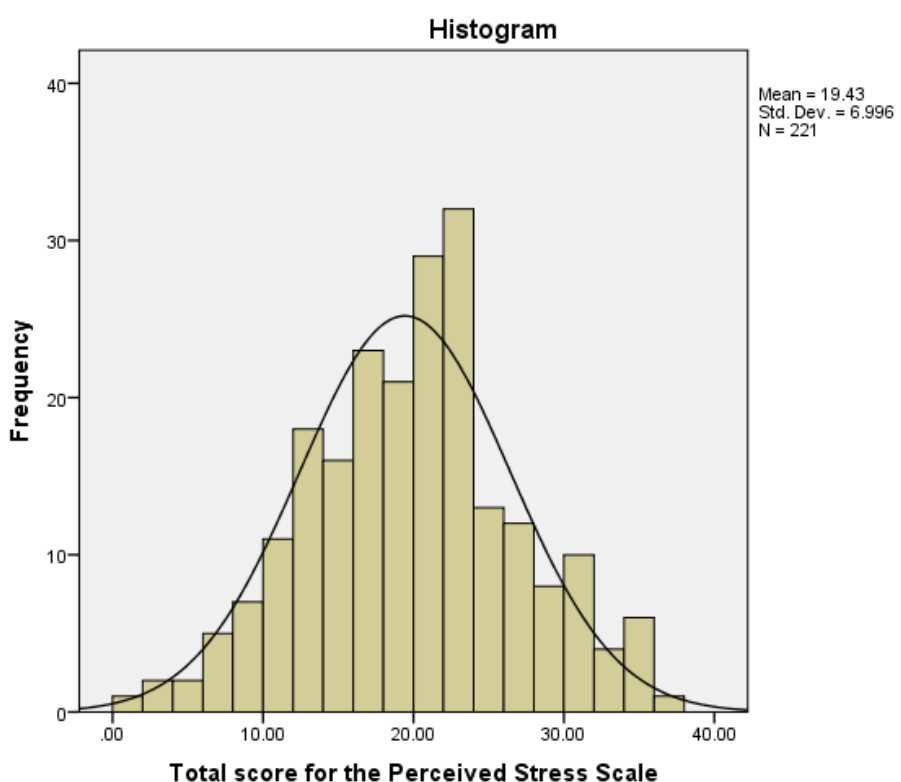

Perceived Stress Scale outliers not included

Total score for the Perceived Stress Scale

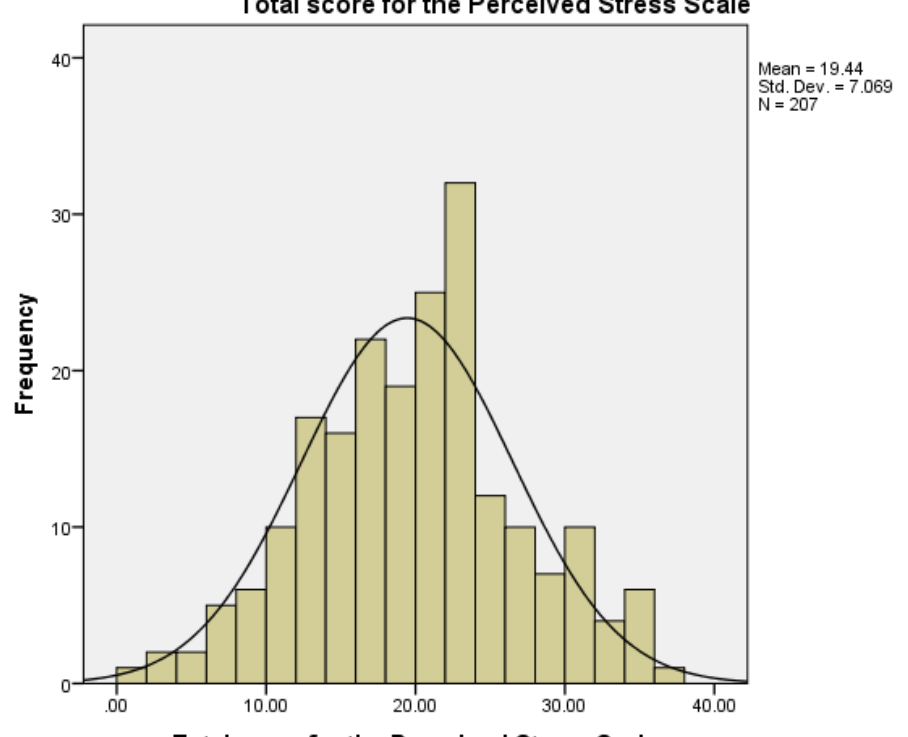

Total score for the Perceived Stress Scale 


\section{Appendix N Q-Q Plots}

BMI outliers included

BMI outliers not included
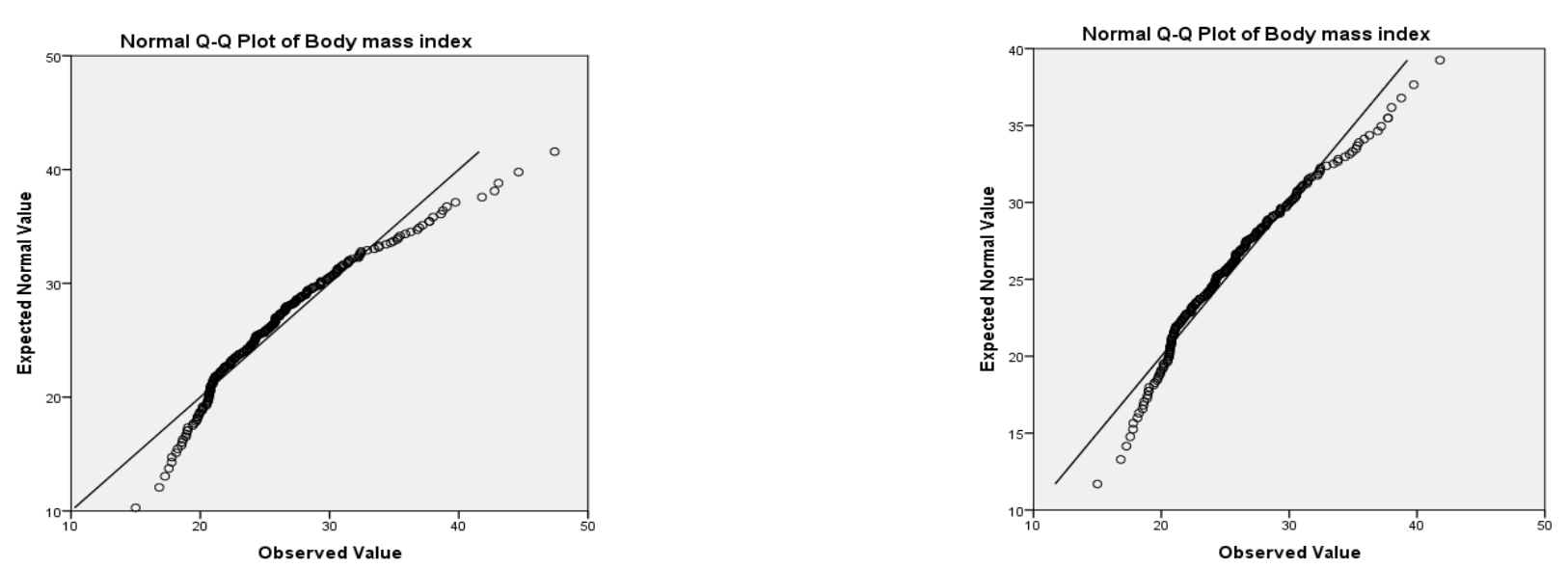

BMI (square root transformation) outliers not included

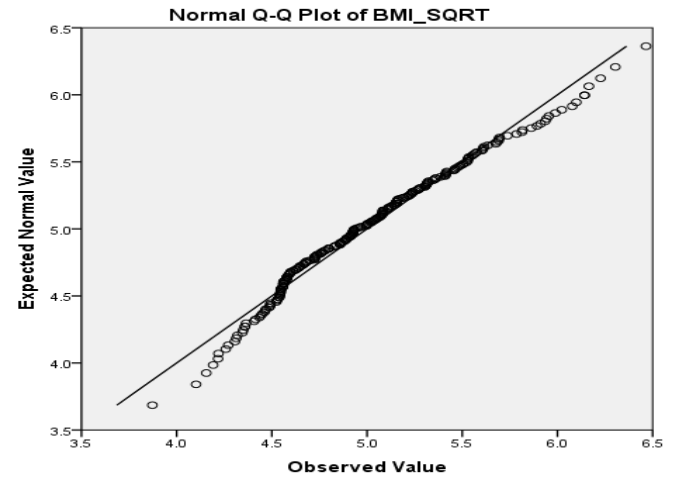


DFI outliers included

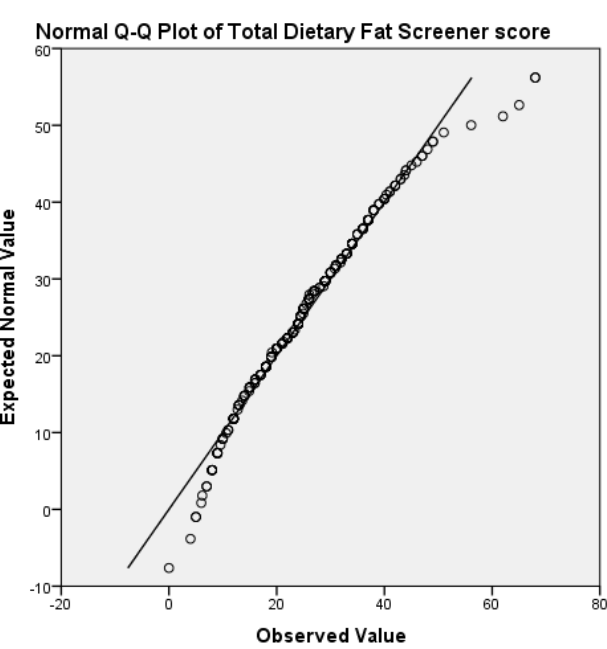

DFI outliers not included

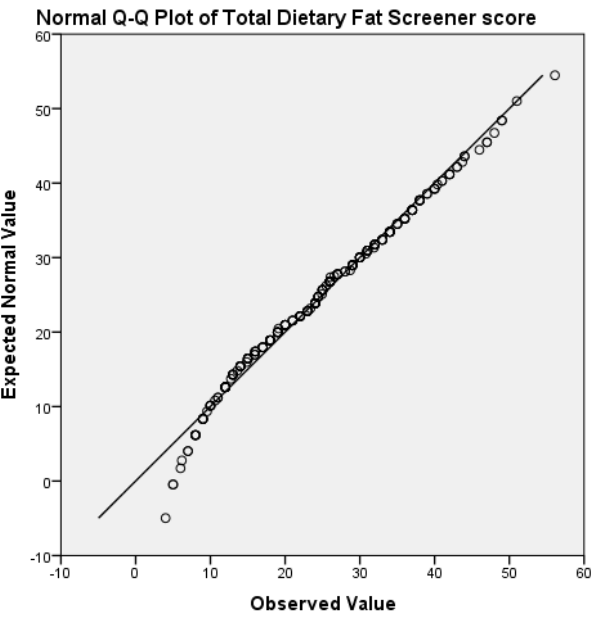


Intrinsic Religiosity Subscale outliers included Normal Q-Q Plot of Total score for the Intrinsic Religiosity Subscale

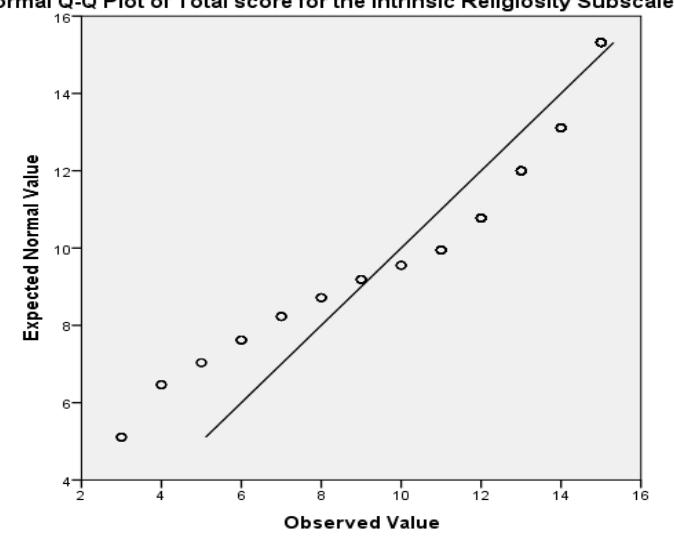

Intrinsic Religiosity Subscale outliers not included

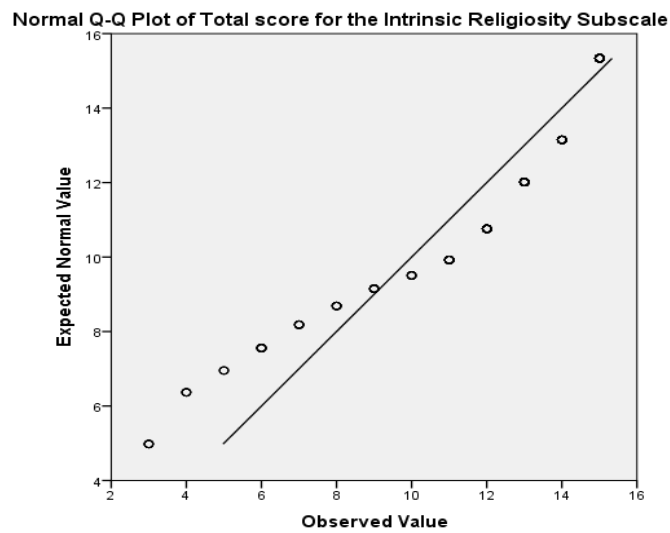

Intrinsic Religiosity Subscale (reflect/logarithm transformation) outliers not included

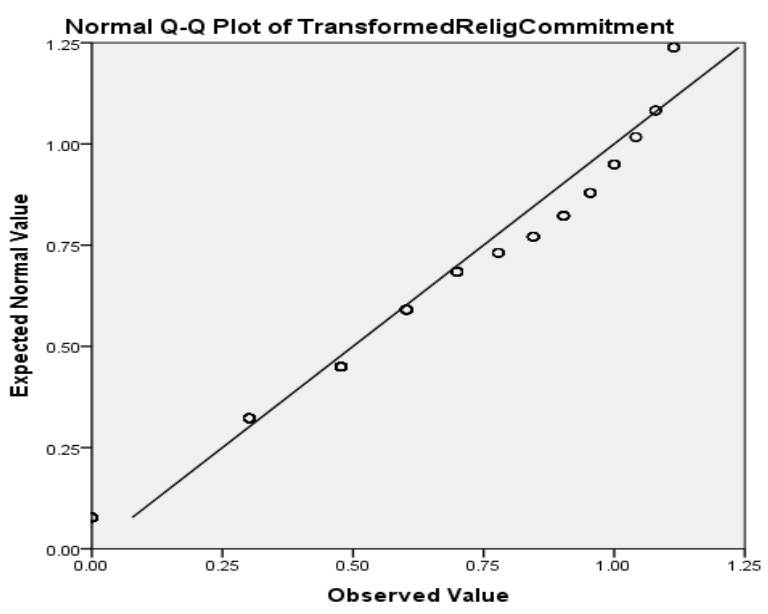


Income Adequacy Scale outliers included

Normal Q-Q Plot of Total score for the Income Adequacy Scale

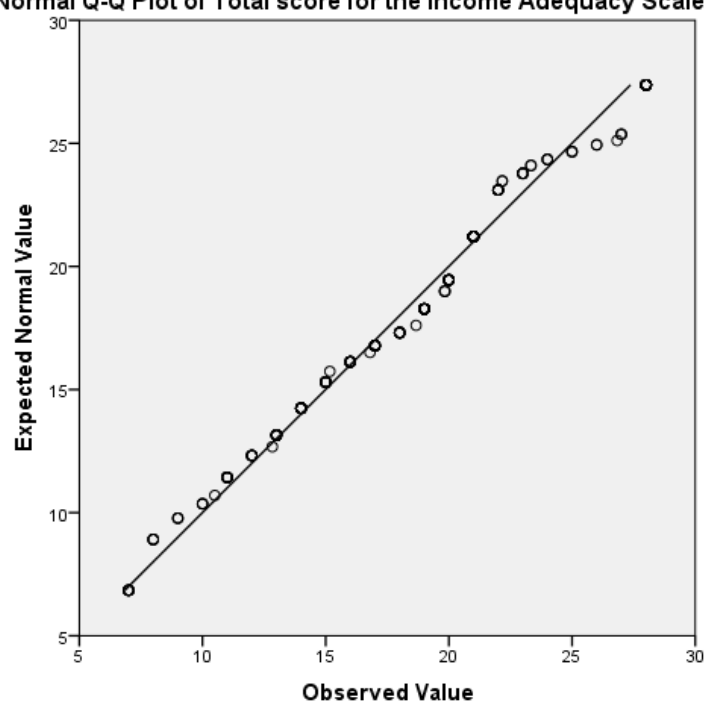

Income Adequacy Scale outliers not included

Normal Q-Q Plot of Total score for the Income Adequacy Scale

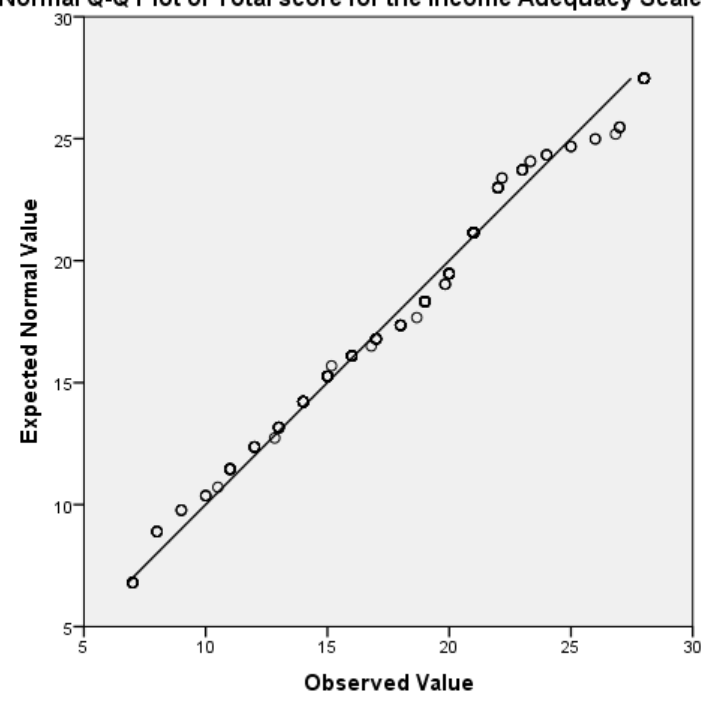


Barriers Subscale outliers included

Normal Q-Q Plot of Total score for the Healthy Foods and Snacks-Barriers

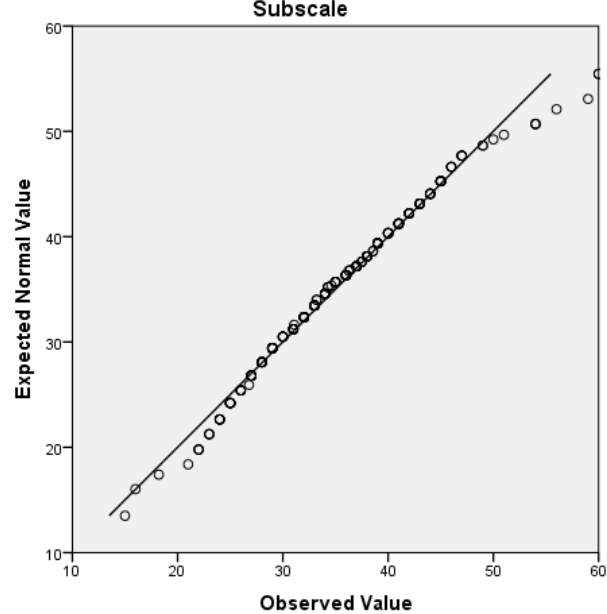

Barriers Subscale outliers not included Normal Q-Q Plot of Total score for the Healthy Foods and Snacks-Barriers

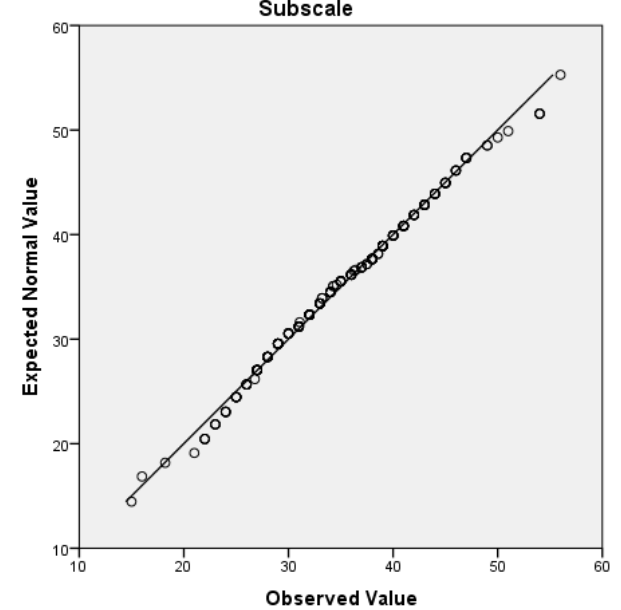


Resisting Relapse Subscale outliers included Normal Q-Q Plot of Total score for the Resisting Relapse Subscale (self-efficacy)

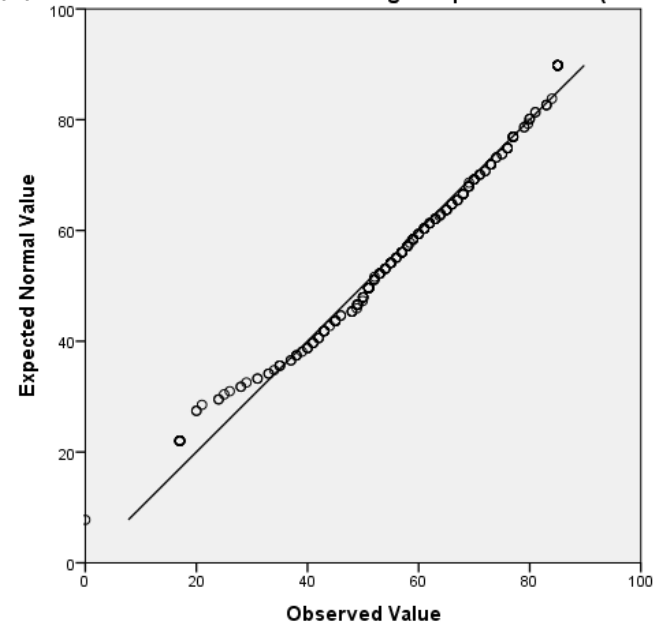

Resisting Relapse Subscale outliers not included

Normal Q-Q Plot of Total score for the Resisting Relapse Subscale (self-efficacy)

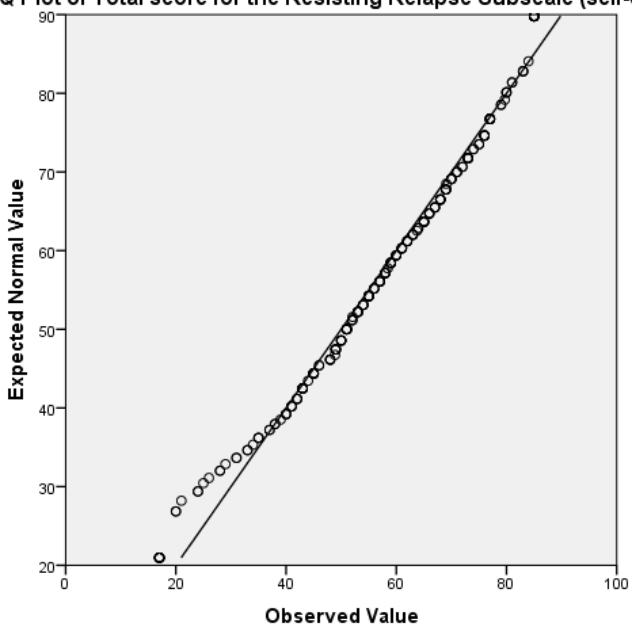


Family Support Scale outliers included

Normal Q-Q Plot of Total score for the Family Support for Heart Healthy Eating

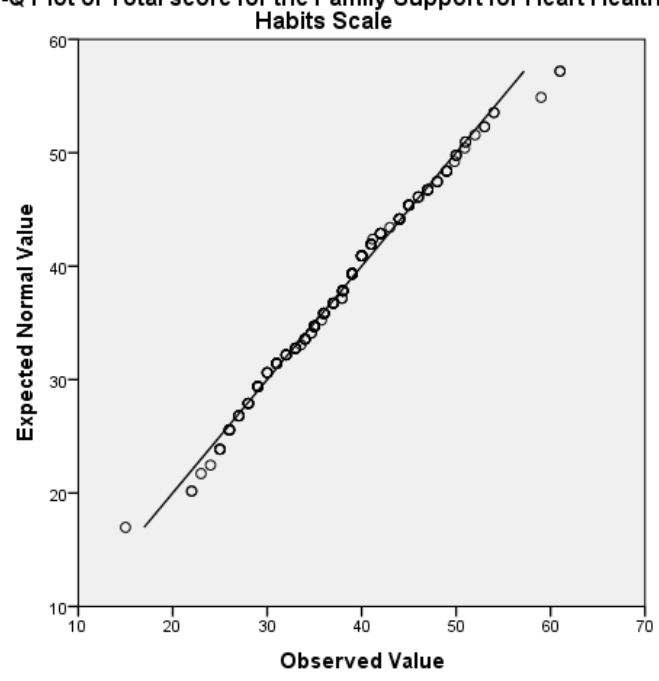

Family Support Scale outliers not included

Normal Q-Q Plot of Total score for the Family Support for Heart Healthy Eating

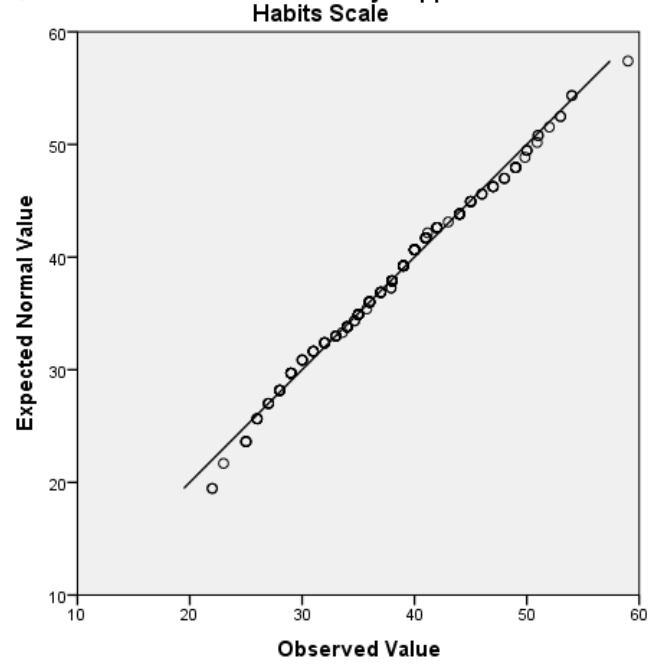


Friend Support Scale outliers included

Normal Q-Q Plot of Total score for the Friend Support for Heart Healthy Eating

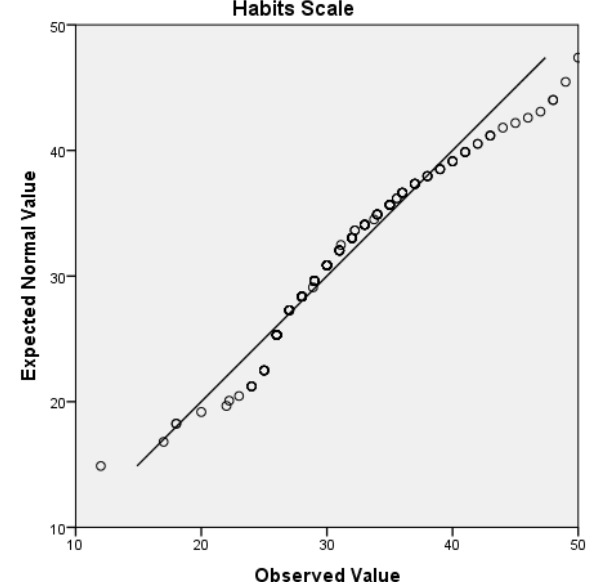

Fiend Support Scale outliers not included Normal Q-Q Plot of Total score for the Friend Support for Heart Healthy Eating

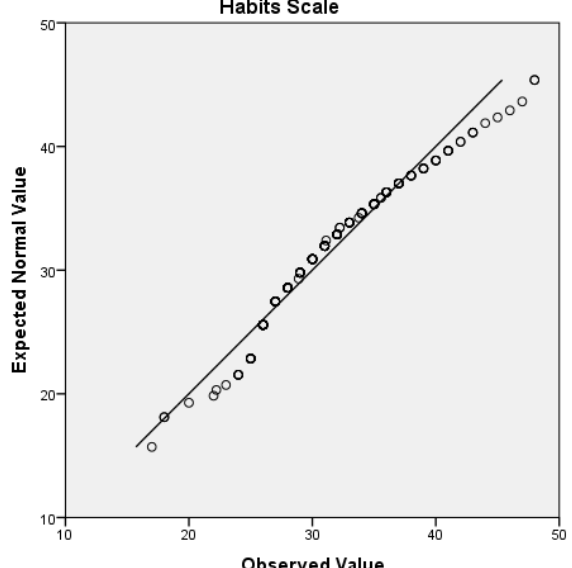

Friend Support Scale (square root transformation) outliers not included

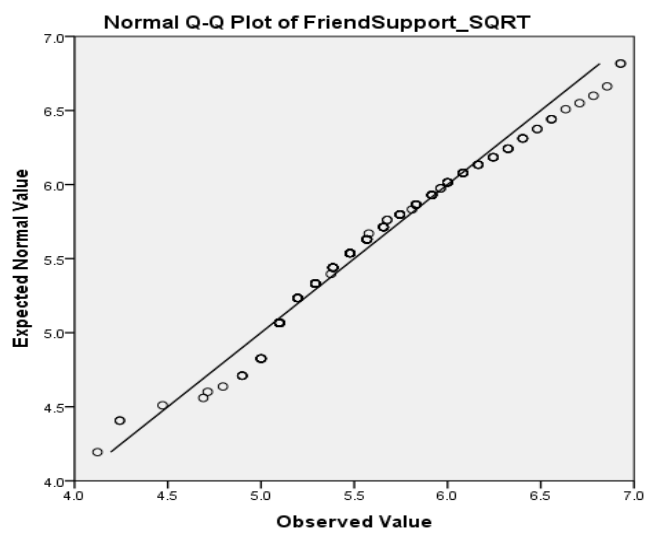


Perceived Stress Scale outliers included Normal Q-Q Plot of Total score for the Perceived Stress Scale

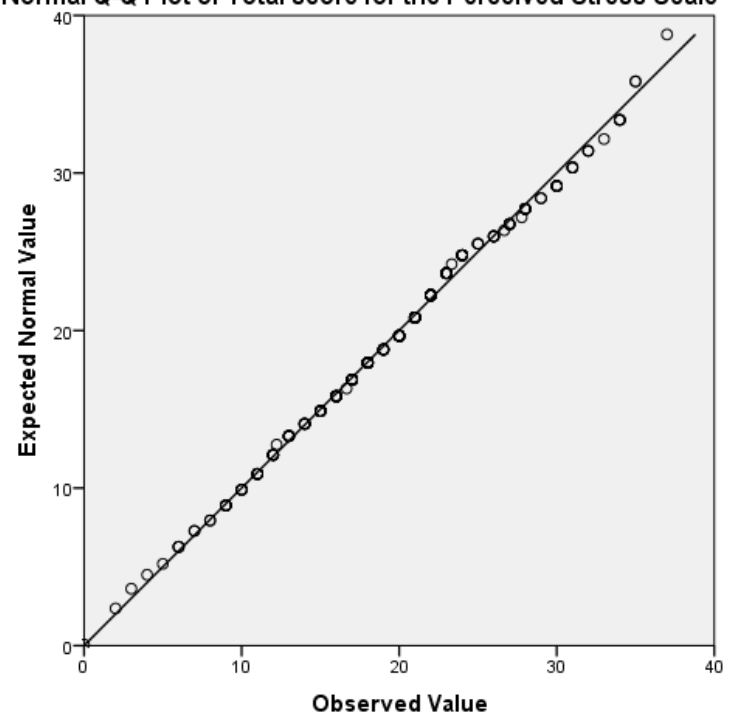

Perceived Stress Scale outliers not included

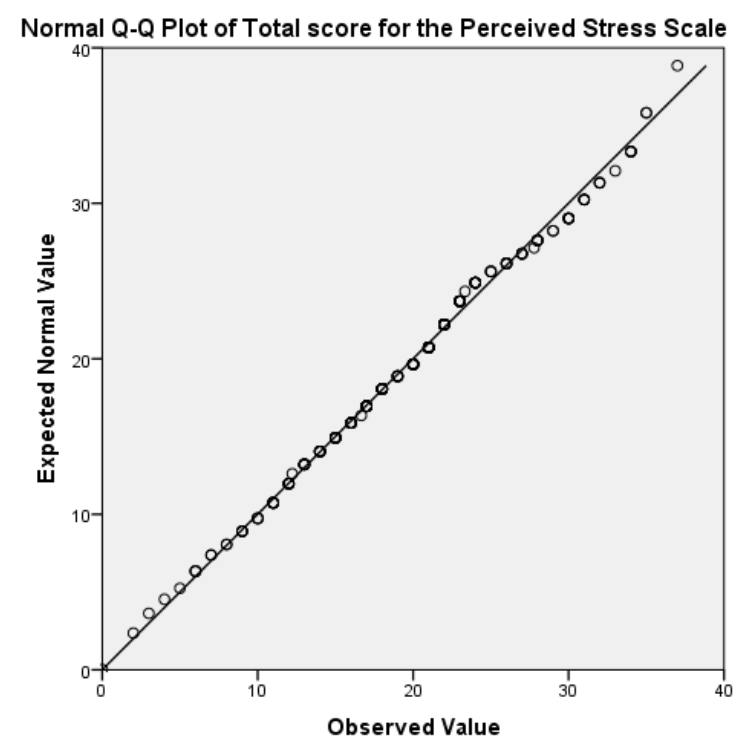




\section{Appendix O IRB Approval for Austin Community College, Huston- Tillotson University, and Xavier University}

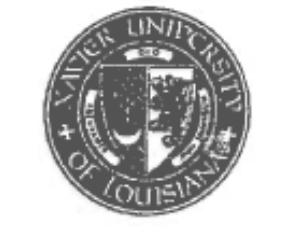

\author{
XAVIER UNIVERSITY OF LOUISIANA \\ DEPARTMENT OF PSYCHOLOGY
}

1 Drexel Drive - Box 115

New Orleans, Louisiana 70125-1098

(504) 520-7400 • Fax: (504) 520-7952
Shalonda Horton, PhD candidate, Principle Investigator

FROM: Charles A. Gramlich, PhD

Chair of the Xavier University IRB

DATE: $\quad$ March 1, 2014

RE: Research Proposal entitled: "Factors Influencing Dietary Fat Intake Among Black Young Adults."

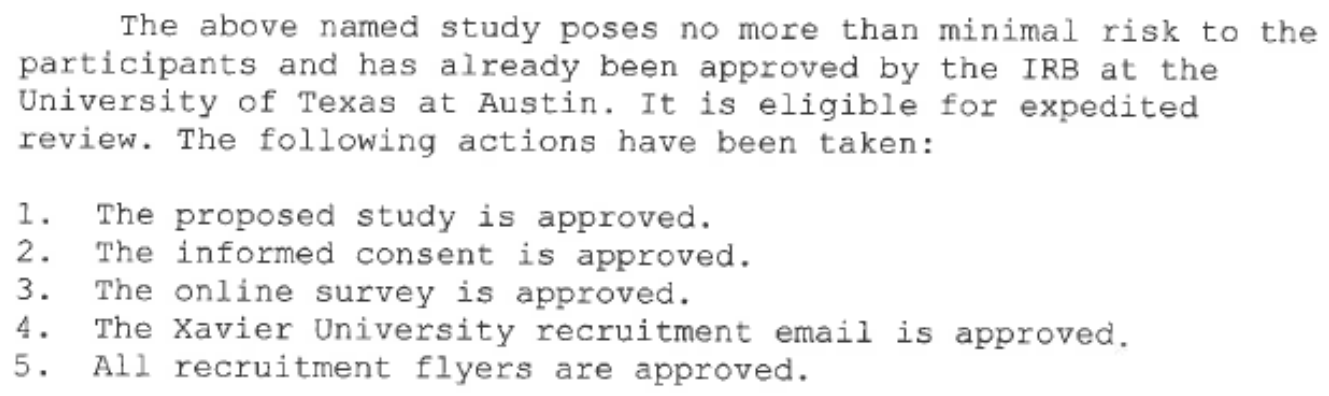


INSTITUTIONAL REVIEW BOARD

HUSTON-TILLOTSON UNIVERSITY

900 Chicon Street, Austin, TX 78702-2795

$512.505 .3019, \cdot$ Fax 512.505 .3198

Date: $7 / 07 / 2014$

PI: Ms. Shalonda E. Horton

Program: Nursing

Institution: University of Texas at Austin

Title: Factors Influencing Dietary Fat Intake Among Black Young Adults

Re: IRB Expedited Approval for Protocol Number 2014-00015

Dear Ms. Horton,

In accordance with Huston-Tillotson research policies and regulations, the Research Standards Committee and Institutional Review Board (IRB) reviewed the above referenced research study and found it met the requirements for approval under the Expedited category noted below for the following period of time; 7/07/2014 to 7/07/2015 Expires 12 a.m. [midnight] of this date. If the research will be conducted at more than one site, you may initiate research at any site from which you have a letter granting you permission to conduct the research. You should retain a copy of the letter in your files. It is to be executed in accordance with the protocol approved by your institution(s) and also by the HustonTillotson University Research Standards Committee and Institutional Review Board.

Please note that the Huston-Tillotson University Research Standards Committee and Institutional Review Board has not obtained the Federalwide Assurance (FWA) from the United States Department of Human and Health Services Office for Human Research Protections (OHRP). Therefore additional approval is needed by an appropriate FWA approved Institutional Review Board for research funded by any federal agency considered under the "Common Rule." Huston-Tillotson University Research Standards Committee and Institutional Review Board does, however, rely upon OHRP for guiding research standards and policies.

\section{Expedited category of approval:}

Research on individual or group characteristics or behavior (including, but not limited to, research on perception, cognition, motivation, identify, language, communication, cultural beliefs or practices, and social behavior) or research employing survey, interview, oral history, focus group, program evaluation, human factors evaluation, or equality assurance methodologies. Note: some research in this category may be exempt from the HHS regulations for the protection of 
buman subjects. 45 CFR 46.101(b)(2) and (b)(3). This listing refers only to resesrch that is not extmpt.

Flease use the consent document included wittin the approved survey and make available the contast inferasation for the Huston-Tiliotson Ualversiry Research Standards Committes and hatitutionsl Revien Bcard should Huston-Tillotsce Uaiversity affliated participants have asy questions conserning their rights.

\section{Responaibilities of the Principal Investigater:}

1) Report immediately amy unanticipated problems or atveree events resulting from this research to the Research Stardaeds Commintes and Instimtiensi Review Bosrd at both your institution(s) and Huston-Tillotson University at the time of occurrence.

2) Submit for review and apporval by the Resasch Standards Committee and Inotitational Review Beord at beth Hustoe-Tillotwen Lniversity and your instibution(s) sll modifications to the protocol or consen form(s). Ensure the propseed changes in the approved research are not applied without pricr review and apcroml, except when necessary to eliminate epperent immediote hazards to the subject. Changos in epproved reseerch, implemented without IRB review and approval, and initiated to eliminate apparent immediate hazands to the subject most be promptly seported to the Besearch Standards Committee and Irstitutional Review Board at both Heston-Tillotson University and your institution(s). Soch changes will be reviewed to deternitine wietter the modifications were consistent with ensuring the subjects continued welfere.

3) Report any significant findings that become known in the course of the research that might affect the willingness of subjects to continve to pertivipate.

4) Ensure that only persons formally approved by the Reseacch Standands Coumittee and Institutional Review Bcard at both Heston-Tillocson University and you instituboo(s) enroll subjects.

5) The approved prolocol for sdministration of the informed consent (as docamented) is to be used for all subjects. Use only a currently approved ocnsent form submitted. Note: approval periods ave for 12 months or less.

6) Protect the confidestiality of all persoas and perscesally identifiable data, and train your staff and collaborakes an policies and procederes for ensuring the privacy and confidentiality of subjects and their infermation.

7 Submit a Continuisg Review application for continuing review by tho Reseasch Standands Commitien and Instituticeal Review Besd at both Huston-Tillotson University and your institution(8). The Contituing Review Applieation must be submitted, reviewed and approved, before the expiration dale.

3) Include the Huskes-Tillesen Universiny Research Standands Commiltex and Institutional Review Bourd study aumber an all funure correspondence reiating wo this protocol. 
human subjects. 45 CFR 46.101(b)(2) and (b)(3). This listing refers only to research that is not exempt.

Please use the consent document included within the approved survey and make available the contact information for the Huston-Tillotson University Research Standards Committee and Institutional Review Board should Huston-Tillotson University affiliated participants have any questions concerning their rights.

\section{Responsibilities of the Principal Investigator:}

1) Report immediately any unanticipated problems or adverse events resulting from this research to the Research Standards Committee and Institutional Review Board at both your institution(s) and Huston-Tillotson University at the time of occurrence.

2) Submit for review and approval by the Research Standards Committee and Institutional Review Board at both Huston-Tillotson University and your institution(s) all modifications to the protocol or consent form(s). Ensure the proposed changes in the approved research are not applied without prior review and approval, except when necessary to eliminate apparent immediate hazards to the subject. Changes in approved research, implemented without IRB review and approval, and initiated to eliminate apparent immediate hazards to the subject must be promptly reported to the Research Standards Committee and Institutional Review Board at both Huston-Tillotson University and your institution(s). Such changes will be reviewed to determine whether the modifications were consistent with ensuring the subjects continued welfare.

3) Report any significant findings that become known in the course of the research that might affect the willingness of subjects to continue to participate.

4) Ensure that only persons formally approved by the Research Standards Committee and Institutional Review Board at both Huston-Tillotson University and your institution(s) enroll subjects.

5) The approved protocol for administration of the informed consent (as documented) is to be used for all subjects. Use only a currently approved consent form submitted. Note: approval periods are for 12 months or less.

6) Protect the confidentiality of all persons and personally identifiable data, and train your staff and collaborators on policies and procedures for ensuring the privacy and confidentiality of subjects and their information.

7) Submit a Continuing Review application for continuing review by the Research Standards Committee and Institutional Review Board at both Huston-Tillotson University and your institution(s). The Continuing Review Application must be submitted, reviewed and approved, before the expiration date.

8) Include the Huston-Tillotson University Research Standards Committee and Institutional Review Board study number on all future correspondence relating to this protocol. 
If you have any questions, please contact the Huston-Tillotson University Chair of the Research Standards Committee and Institutional Review Board by phone at (512) 505-3019 or via e-mail at iksoni@htu.edu.

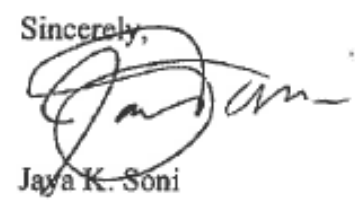

Chair of Research Standards Committee and Institutional Review Board Huston-Tillotson University 
5930 Middle Fiskville Road • Austin, Texas 78752-4390 - Phone 512/223-7601 • Email oieinfo@austincc.edu • Fax 512/223-7029

\author{
Austin Community College \\ Research Review Committee
}

Letter of Agreement

DATE: June 2, 2014

TO: $\quad$ Shalonda Horton

The University of Texas at Austin

School of Nursing

1710 Red River

Austin, TX 78701

FROM: $\quad$ Soon O. Merz

On behalf of the Research Review Committee of Austin Community College, I am pleased to inform you that the proposal you submitted, "Factors Influencing Dietary Fat Intake Among Black Young Adults" has been approved. The committee, however, approved this research with the qualifier that you can recruit subjects at ACC with flyers but not via student emails.

If you wish to pursue this proposal, please sign and return this letter to the Office of Institutional Effectiveness and Accountability by June 20,2014. All ACC-based research must be completed within one year of this agreement, unless otherwise stated.

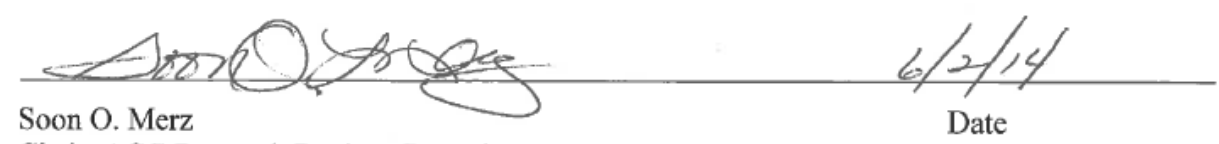

Chair, ACC Research Review Committee

Date

VP for Effectiveness and Accountability 


\section{Researcher's Statement}

I have read the Research Review Process and agree to abide by the guidelines specified there. listed above.

I understand that my Research Proposal has been approved contingent upon the modifications

I understand that approval of this project does not imply Austin Community College's endorsement of either the project or it results.

I understand that Austin Community College is not responsible for any debts that I may incur as part of this project nor will it provide consumable resources.

I will provide a copy of the results of this study to the Research Review Committee of Austin Community College.

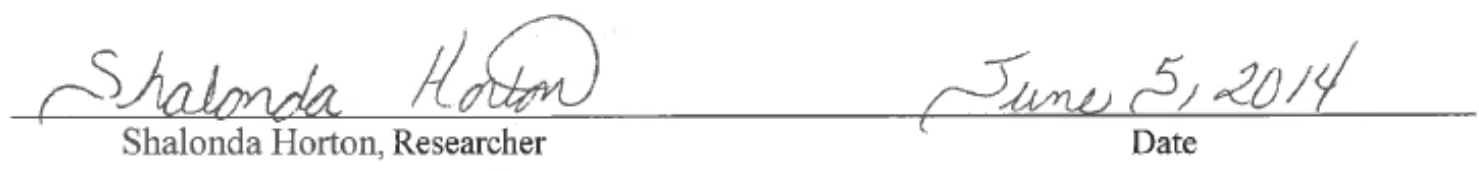




\section{References}

Abbott, P., Davison, J., Moore, L., \& Rubinstein, R. (2010). Barriers and enhancers to dietary behaviour change for Aboriginal people attending a diabetes cooking course. Health Promotion Journal of Australia, 21(1), 33-38.

AbuSabha, R. \& Achterberg, C. (1997). Review of self-efficacy and locus of control for nutrition- and health-related behavior. Journal of the American Dietetic Association, 97(10), 1122-1132. doi:10.1016/S0002-8223(97)00273-3

Adler, N. E. \& Rehkopf, D. H. (2008). U.S. disparities in health: Descriptions, causes, and mechanisms. Annual Review of Public Health, 29, 235-252. doi:

10.1146/annurev.publhealth.29.020907.090852

Affenito, S. G., Franko, D. I., Striegel-Moore, R. H., \& Thompson, D. (2012). Behavioral determinants of obesity: Research findings and policy implications. Journal of Obesity, 2012, 4 pages, Article ID 150732. Online publication. doi:10.1155/2012/150732. Retrieved from http://dx.doi.org/10.1155/2012/150732

Aggarwal, B., Liao, M., Allegrante, J. P., \& Mosca, L. (2010). Low social support level is associated with non-adherence to diet at 1 year in the Family Intervention Trial for Heart Health (FIT Heart). Journal of Nutrition Education and Behavior, 42(6), 380-388. doi: 10.1016/j.jneb.2009.08.006

Aguinis, H. (2004). Regression analysis for categorical moderators. New York, NY: The Guilford Press.

Airhihenbuwa, C. O., Kumanyika, S., Agurs, T. D., Lowe, A., Saunders, D., \& Morssink, C. B. (1996). Cultural aspects of African American eating patterns. Ethnicity \& Health, 1(3), 245-260.

Allen, R. S., Harris, G. M., Crowther, M. R., Oliver, J. S., Cavanaugh, R., \& Phillips, L. L. (2013). Does religiousness and spirituality moderate the relations between 
physical and mental health among aging prisoners? International Journal of Geriatric Psychiatry, 28(7), 710-717. doi: 10.1002/gps.3874

Allison, M. \& Campbell, C. (2009). "Maybe it could be a heart attack .. . but I'm only 31": Young men's lived experience of myocardial infarction —an exploratory study. American Journal of Men's Health, 3(2), 116-125. doi: $10.1177 / 1557988307308519$

Amazon.com. (2013). Amazon gift card-e-mail. Retrieved from https://www.amazon.com/gp/product/B004LLIKVU/gcrnsts?ie=UTF8\&keywords $=$ amazon\%20electronic\%20gift\%20 $\operatorname{card} \& q i d=1366219807 \&$ ref__esr_1_2\&sr=8-2

American College Health Association. (2007). National College Health Assessment Spring 2006 Reference Group Data Report (Abridged): The American College Association, Journal of American College Health, 55(4), 195-206. doi:

10.3200/JACH.55.4.195-206

American Heart Association. (2011, June 29). Saturated fats. Retrieved from http://www.heart.org/HEARTORG/GettingHealthy/FatsAndOils/Fats101/Saturate d-Fats_UCM_301110_Article.jsp

American Heart Association. (2012, October 11). What your cholesterol levels mean. Retrieved from http://www.heart.org/HEARTORG/Conditions/What-YourCholesterol-Levels-Mean_UCM_305562_Article.jsp

American Heart Association. (2013, February 7). Southern diet could raise your risk of stroke. American Stroke Association Meeting Report - Abstract 144. Retrieved from http://newsroom.heart.org/news/southern-diet-could-raise-your-risk-ofstroke 
Anderson, E. S., Winett, R. A., Wojcik, J. R., \& Williams, D. M. (2010). Social cognitive mediators of change in a group randomized nutrition and physical activity intervention: Social support, self-efficacy, outcome expectations and selfregulation in the guide-to-health trial. Journal of Health Psychology, 15(1), 21-32. doi: $10.1177 / 1359105309342297$

Anderson, J. C. (2012, December 13). Byron Hurt's 'Soul Food Junkies' film explores how southern fare got its soul. Huffington Post. Retrieved from http://www.huffingtonpost.com/2012/12/13/byron-hurt-soul-foodjunkies_n_2295860.html

Anderson-Bill, E. S., Winett, R. A., \& Wojcik, J. R. (2011). Social cognitive determinants of nutrition and physical activity among web-health users enrolling in an online intervention: The influence of social support, self-efficacy, outcome expectations, and self-regulation. Journal of Medical Internet Research, 13(1), e28. Online publication. doi: 10.2196/jmir.1551. Retrieved from http://www.jmir.org/2011/1/e28/

Ano, G. G. \& Vasconcelles, E. B. (2005). Religious coping and psychological adjustment to stress: A meta-analysis. Journal of Clinical Psychology, 61(4), 461-480. doi: 10.1002/jclp.20049

Armitage, C. J. \& Conner, M. (1999). Distinguishing perceptions of control from selfefficacy: Predicting consumption of a low-fat diet using the Theory of Planned Behavior. Journal of Applied Social Psychology, 29(1), 72-90. doi: 10.1111/j.1559-1816.1999.tb01375.x

Arnett, J. J. (2000). Emerging adulthood: A theory of development from the late teens through the twenties. American Psychologist, 55(5), 469-480. doi: 10.1037/0003066X.55.5.469

Arnett, J. J. (2004). Emerging adulthood: The winding road from the late teens through the twenties. Oxford, NY: Oxford University Press. 
Arnett, J. J. (2010). Adolescence and emerging adulthood: A cultural approach. Upper Saddle River, NJ: Pearson Education, Inc.

Bailey, E. J. (2006). Food choice and obesity in Black America: Creating a new cultural diet. Westport, CT: Praeger Publishers.

Baker, A. H. \& Wardle, J. (2003). Sex differences in fruit and vegetable intake in older adults. Appetite, 40(3), 269-275. doi:10.1016/S0195-6663(03)00014-X

Bandura, A. (1986). Social foundations of thought and action: A Social Cognitive Theory. Engelwood Cliffs, NJ: Prentice-Hall.

Baqutayan, S. (2011). Stress and social support. Indian Journal of Psychological Medicine, 33(1), 29-34. dio: 10.4103/0253-7176.85392

Baron, R. M. \& Kenny, D. A. (1986). The moderator-mediator variable distinction in social psychological research: Conceptual, strategic, and statistical considerations. Journal of Personality and Social Psychology, 51(6), 1173-1182.

Baskin, M. L., Odoms-Young, A. M., Kumanyika, S. K., \& Ard, J. D. (2009). Nutrition and obesity issues for African Americans. In R. L. Braithwaite, S. E. Taylor, \& H. M. Treadwell (Eds.), Health issues in the Black community (3rd ed., pp. 431-460). San Francisco, CA: Jossey-Bass.

Becker, H. \& Stuifbergen, A. (2004). What makes it so hard? Barriers to health promotion experienced by people with multiple sclerosis and polio. Family \& Community Health, 27(1), 75-85.

Bennett, J. A. (2000). Focus on research methods mediator and moderator variables in nursing research: Conceptual and statistical differences. Research in Nursing \& Health, 23, 415-420.

Berg, C. J., An, L. C., \& Ahluwalia, J. S. (2013). Dietary fat intake and exercise among two- and four-year college students: Differences in behavior and psychosocial 
factors. Community College Journal of Research and Practice, 37(5), 388-396. doi: 10.1080/10668921003609285

Berman, J. (5 February 2014). It's a fact that women get paid less than men. Stop debating. Huffington Post. Retrieved from http://www.huffingtonpost.com/jillianberman/yes-by-any-way-you-measur_b_4725356.html

Berrigan, D., Dodd, K., Troiano, R. P., Krebs-Smith, S. M., \& Barbash, R. B. (2003). Patterns of health behavior in U.S. adults. Preventive Medicine, 36, 615-623. doi: 10.1016/S0091-7435(02)00067-1

Beydoun, M. A., Powell, L. M., \& Wang, Y. (2008). The association of fast food, fruit and vegetable prices with dietary intakes among US adults: Is there modification by family income? Social Science \& Medicine, 66(11), 2218-2229. doi: 10.1016/j.socscimed.2008.01.018

Beydoun, M. A. \& Wang, Y. (2008). How do socio-economic status, perceived economic barriers and nutritional benefits affect quality of dietary intake among US adults? European Journal of Clinical Nutrition, 62(3), 303-313. doi:10.1038/sj.ejen.1602700

Bibbins-Domingo, K. \& Peña, M. B. (2010). Caring for the "young invincibles". Journal of General Internal Medicine, 25(7), 642-643. doi: 10.1007/s11606-010-1388-8

Binkley, J. K., Eales, J., \& Jekanowski, M. (2000). The relation between dietary change and rising US obesity. International Journal of Obesity, 24(8), 1032-1039.

Björntorp, P. (2001). Do stress reactions cause abdominal obesity and comorbidities? Obesity Reviews, 2(2), 73-86. doi: 10.1046/j.1467-789x.2001.00027.x

Block, G., Gillespie, C., Rosenbaum, E. H., \& Jenson, C. (2000). A rapid food screener to assess fat and fruit and vegetable intake. American Journal of Preventive Medicine, 18(4), 284-288. 
Bloom, J. R. (1990). The relationship of social support and health. Social Science \& Medicine, 30(5), 635-637.

Blundell, J. E., Burley, V. J., Cotton, J. R., \& Lawton, C. L. (1993). Dietary fat and the control of energy intake: Evaluating the effects of fat on meal size and postmeal satiety. The American Journal of Clinical Nutrition, 57(5 Suppl), 772S-778S.

Blundell, J. E. \& Macdiarmid, J. I. (1997). Passive overconsumption. Fat intake and short-term energy balance. Annuals of the New York Academy of Sciences, 827(1), 392-407. doi: 10.1111/j.1749-6632.1997.tb51850.x

Blundell, J. E. \& Stubbs, J. (2004). Diet composition and the control of food intake in humans. In Bray, G. A. \& Bouchard, C. (Eds.), Handbook of obesity: Etiology and pathophysiology (2nd ed., pp. 427-460). New York, NY: Marcel Dekker, Inc.

Bonhomme, J. J. E. \& Young, A. M. W. (2009). The health status of Black men. In R. L. Braithwaite, S. E. Taylor, \& H. M. Treadwell (Eds.), Health issues in the Black community (3rd ed., pp. 73-94). San Francisco, CA: Jossey-Bass.

Boozer, C. N., Schoenbach, G., \& Atkinson, R. L. (1995). Dietary fat and adiposity: A dose-response relationship in adult male rats fed isocalorically. American Journal of Physiology, 268, E546-E550.

Bortsov, A., Liese, A. D., Bell, R. A., Dabelea, D., D’Agostino, R. B. Jr., Hamman, R. F., ... Mayer-Davis, E. J. (2011). Correlates of dietary intake in youth with diabetes: Results from the SEARCH for Diabetes in Youth Study. Journal of Nutrition Education and Behavior, 43(2), 123-129. doi: 10.1016/j.jneb.2009.12.007

Bovell-Benjamin, A. C., Dawkins, N., Pace, R. D., \& Shikany, J. M. (2009). Use of focus groups to understand African-Americans' dietary practices: Implications for modifying a food frequency questionnaire. Preventive Medicine, 48, 549-554. doi:10.1016/j.ypmed.2009.03.006 
Bovell-Benjamin, A., Dawkins, N., Pace, R., \& Shikany, J. M. (2010). Dietary consumption practices and cancer risk in African Americans in the rural south. Journal of Health Care for the Poor and Underserved, 21(3), 57-75. doi: 10.1353/hpu.0.0361

Bowen-Reid, T. L. \& Harrell, J. P. (2002). Racist experiences and health outcomes: An examination of spirituality as a buffer. Journal of Black Psychology, 28, 18-36. dio: $10.1177 / 0095798402028001002$

Bowman, R. L. \& DeLucia, J. L. (1992). Accuracy of self-reported weight: A metaanalysis. Behavior Therapy, 23(4), 637-655.

Bowring, A. L., Peeters, A., Freak-Poli, R., Lim, M. S., Gouillou, M., \& Hellard, M. (2012). Measuring the accuracy of self-reported height and weight in a community-based sample of young people. BMC Medical Research Methodology, 12(175). Online publication. doi: 10.1186/1471-2288-12-175. Retrieved from http://www.biomedcentral.com/1471-2288/12/175

Braithwaite, R. L., Taylor, S. E., \& Treadwell, H. M. (Eds.). (2009). Health issues in the Black community (3rd ed.). San Francisco, CA: Jossey-Bass.

Bray, G. A., Paeratakul, S., \& Popkin, B. M. (2004). Dietary fat and obesity: A review of animal, clinical, and epidemiological studies. Physiology \& Behavior, 83(4), 549555. doi:10.1016/j.physbeh.2004.08.039

Bray, G. A. \& Popkin, B. M. (1998). Dietary fat intake does affect obesity! The American Journal of Clinical Nutrition, 68(6), 1157-1173.

Brener, N. D., McManus, T., Galuska, D. A., Lowry, R., \& Wechsler, H. (2003). Reliability and validity of self-reported height and weight among high school students. Journal of Adolescent Health, 32, 281-287. doi:10.1016/S1054139X(02)00708-5

Brown, S. C., Geiselman, P. J., \& Broussard, T. (2010). Cardiovascular risk in African American Women attending historically Black colleges and universities: The role 322 
of dietary patterns and food preferences. Journal of Health Care for the Poor and Underserved, 21, 1184-1193. doi: 10.1353/hpu.2010.0933

Brown, S. E. D. (2009). Health promotion behaviors among African American women. (Doctoral dissertation). Retrieved from http://scholarlyrepository.miami.edu/cgi/viewcontent.cgi?article=1204\&context= oa_dissertations

Bruening, K. S., Gilbride, J. A., Passannante, M. R., \& McClowry, S. (1999). Dietary intake and health outcomes among young children attending 2 urban day-care centers. Journal of the American Dietetic Association, 99(12), 1529-1535.

Brug, J. (2008). Determinants of healthy eating: Motivation, abilities and environmental opportunities. Family Practice, 25(Suppl 1), i50-i55. doi:

10.1093/fampra/cmn063

Brug, J., Van Assema, P., Kok, G., Lenderink, T., \& Glanz, K. (1994). Self-rated dietary fat intake: Association with objective assessment of fat, psychosocial factors, and intention to change. Journal of Nutrition Education, 26(5), 218-223.

Bufford, R. K., Paloutzian, R. F., \& Ellison, C. W. (1991). Norms for the Spiritual Wellbeing Scale. Journal of Psychology and Theology, 19(1), 56-70.

Bull, S., Eakin, E., Reeves, M., \& Kimberly, R. (2006). Multi-level support for physical activity and healthy eating. Journal of Advanced Nursing, 54(5), 585-593. doi: 10.1111/j.1365-2648.2006.03861.x

Burke, V., Beilin, L. J., Cutt, H. E., Mansour, J., \& Mori, T. A. (2008). Moderators and mediators of behaviour change in a lifestyle program for treated hypertensives: A randomized controlled trial (ADAPT). Health Education Research, 23(4), 583591. doi: 10.1093/her/cym047

Business.com. (2013). Marketing your business on Craigslist. Retrieved from http://www.business.com/guides/marketing-your-business-on-craigslist-1382 
Callaghan, D. M. (2003). Health-promoting self-care behaviors, self-care self-efficacy, and self-care agency. Nursing Science Quarterly, 16(3), 247-254. doi:

$10.1177 / 0894318403016003016$

Callaghan, D. (2006). Basic conditioning factors' influences on adolescents' healthy behaviors, self-efficacy, and self-care. Issues in Comprehensive Pediatric Nursing, 29(4), 191-204. doi:10.1080/01460860601087156

Calvert, W. J. \& Isaac-Savage, E. P. (2013). Motivators and barriers to participating in health promotion behaviors in Black men. Western Journal of Nursing Research, 35(7), 829-848. doi: doi: 10.1177/0193945913480429.

Campbell, M. K., Hudson, M. A., Resnicow, K., Blakeney, N., Paxton, A., \& Baskin, M. (2007). Church-based health promotion interventions: Evidence and lessons learned. Annual Review of Public Health, 28, 213-234. dio:

10.1146/annurev.publhealth.28.021406.144016

Canfi, A., Gepner, Y., Schwarzfuchs, D., Golan, R., Shahar, D. R., Fraser, D., ... Shai, I. (2011). Effect of changes in the intake of weight of specific food groups on successful body weight loss during a multi-dietary strategy intervention trial. Journal of the American College of Nutrition, 30(6), 491-501.

Carlson, K. A. \& Gould, B. W. (1994). The role of health knowledge in determining dietary fat intake. Applied Economic Perspectives and Policy, 16(3), 373-386. doi: 10.2307/1349697

Cartwright, M., Wardle, J., Steggles, N., Simon, A. E., Croker, H., \& Jarvis, M. J. (2003). Stress and dietary practices in adolescents. Health Psychology, 22(4), 362-369. doi: 10.1037/0278-6133.22.4.362

Centers for Disease Control and Prevention. (2011, January 14). CDC health dispartities and inequalities report-United States, 2011. Morbidity and Mortality Weekly Report, 60(Supplement), 1-116. Retreived from http://www.cdc.gov/mmwr/pdf/other/su6001.pdf 
Centers for Disease Control and Prevention. (2012, April 27). Overweight and obesity: Causes and consequences. Retrieved from http://www.cdc.gov/obesity/adult/causes/index.html

Centers for Disease Control and Prevention. (2014a, September 3). Overweight and obesity: Childhood obesity facts. Retrieved from http://www.cdc.gov/obesity/data/childhood.html

Centers for Disease Control and Prevention. (2014b, September 4). Overweight and obesity: Prevalence of self-reported obesity among Non-Hispanic Black adults by state, BRFSS, 2011-2013. Retrieved from http://www.cdc.gov/obesity/data/tablenon-hispanic-black.html

Centers for Disease Control and Prevention. (2014c, September 9). Overweight and obesity: Adult obesity facts. Retrieved from http://www.cdc.gov/obesity/data/trends.html

Centers for Disease Control and Prevention. (2015a February 6). FastStats: Leading causes of death. Retrieved from http://www.cdc.gov/nchs/fastats/lcod.htm

Centers for Disease Control and Prevention. (2015b February 23). Healthy weight - it's not a diet, it's a lifestyle! About BMI for adults. Retrieved from http://www.cdc.gov/healthyweight/assessing/bmi/adult_bmi/

Chae, D. H., Lincoln, K. D., \& Jackson, J. S. (2011). Discrimination, attribution, and racial group identification: Implications for psychological distress among Black Americans in the National Survey of American Life (2001-2003). American Journal of Orthopsychiatry, 81(4), 498-506. doi: 10.1111/j.19390025.2011.01122.x

Chang, E. C., Banks, K. H., \& Watkins, A. F. (2004). How adaptive and maladaptive perfectionism relate to positive and negative psychological functioning: Testing a stress-mediation model in Black and White female college students. Journal of Counseling Psychology, 51(1), 93-102. doi: 10.1037/0022-0167.51.1.93 
Chang, M., Brown, R. L., Baumann, L. J., \& Nitzke, S. A. (2008). Self-efficacy and dietary fat reduction behaviors in obese African-American and White mothers. Obesity, 16(5), 992-1001. doi:10.1038/oby.2008.20

Chatters, L. M. (2000). Religion and health: Public health research and practice. Annual Review of Public Health, 21, 335-367. doi: 10.1146/annurev.publhealth.21.1.335

Chatters, L., M., Taylor, R. J., Jackson, J. S., \& Lincoln, K. D. (2008). Religious coping among African Americans, Caribbean Blacks and non-Hispanic Whites. Journal of Community Psychology, 36(3), 371-386. doi: 10.1002/jcop.20202

Chester, D. N., Himburg, S. P., \& Weatherspoon, L. J. (2006). Spirituality of AfricanAmerican women: Correlations to health-promoting behaviors. Journal of National Black Nurses Association, 17(1), 1-8.

Chung, K. (2010). Moderation effects of spirituality on stress and health. (Doctoral dissertation). Retrieved from http://repository.asu.edu/attachments/56052/content/Chung_asu_0010E_10026.pd $\mathrm{f}$

Clark, N. M. \& Dodge, J. A. (1999). Exploring self-efficacy as a predictor of disease management. Health Education \& Behavior, 26(1), 72-89. doi: 10.1177/109019819902600107

Close, A. G. (Ed.). (2012). Online consumer behavior: Theory and research in social media, advertising, and e-tail. New York, NY: Taylor and Francis Group.

Coates, R. J., Serdula, M. K., Byers, T., Mokdad, A., Jewell, S., Leonard, S. B., ... Block, G. (1995). A brief, telephone-administered food frequency questionnaire can be useful for surveillance of dietary fat intakes. The Journal of Nutrition, 125(6), 1473-1483.

Cohen, A. B. \& Koenig, H. G. (2003). Religion, religiosity and spirituality in the biopsychosocial model of health and aging. Ageing International, 28(3), 215-241. 
Cohen, J. I. (2000). Stress and mental health: A biobehavioral perspective. Issues in Mental Health Nursing, 21(2), 185-202.

Cohen, S., Kamarck, T., \& Mermelstein, R. (1983). A global measure of perceived stress. Journal of Health and Social Behavior, 24(4), 385-396.

Connell, C. M. \& D’Augelli, A. R. (1990). The contribution of personality characteristics to the relationship between social support and perceived physical health. Health Psychology, 9(2), 192-207.

Contrada, R. J. \& Baum, A. (Eds.). (2011). The handbook of stress science: Biology, psychology, and health. New York, NY: Springer Publishing Company, LLC.

Coruh, B., Ayele, H., Pugh, M., \& Mulligan, T. (2005). Does religious activity improve health outcomes? A critical review of the recent literature. Explore, 1(3), 186-191. doi: 10.1016/j.explore.2005.02.001

Cotton, S., Zebracki, K., Rosenthal, S. L., Tsevat, J., \& Drotar, D. (2006). Religion/spirituality and adolescent health outcomes: A review. Journal of Adolescent Health, 38(4), 472-480. doi:10.1016/j.jadohealth.2005.10.005

Coulston, A. M., Boushey, C. J., \& Ferruzzi, M. G. (Eds.). (2013). Nutrition in the prevention and treatment of disease (3rd ed.). London, UK: Elsevier Inc.

Coulston, A. M., Rock, C. L., \& Monsen, E. R. (Eds.). (2001). Nutrition in the prevention and treatment of disease. San Diego, CA: Academic Press.

Craig, B. M. \& Adams, A. K. (2009). Accuracy of body mass index categories based on self-reported height and weight among women in the United States. Maternal and Child Health Journal, 13(4), 489-496. doi: 10.1007/s10995-008-0384-7

Crump, S. R., Taylor, B. D., Sung, J. F. C., Burley, L., Sheats, J., Murphy, F. G., \& Caplan, L. (2006). Dietary intake to reduce cancer risk among African American women in public housing: Do sociodemographic factors make a difference? Ethnicity \& Disease, 16(4), 963-70. 
Dacosta, K. O. \& Wilson, J. F. (1996). Food preferences and eating attitudes in three generations of Black and White women. Appetite, 27, 183-191.

Dallman, M. F. (2010). Stress-induced obesity and the emotional nervous system. Trends in Endocrinology and Metabolism, 21(3), 159-65. doi: 10.1016/j.tem.2009.10.004

Darmon, N. \& Drewnowski, A. (2008). Does social class predict diet quality? The American Journal of Clinical Nutrition, 87(5), 1107-1117.

Davidson, J. (2015, February 4). Americans less stress-Except when it comes to money. Time. Retrieved from http://time.com/money/3695478/stress-america-studymoney/

Debnam, K., Holt, C. L., Clark, E. M., Roth, D. L., \& Southward, P. (2012). Relationship between religious social support and general social support with health behaviors in a national sample of African Americans. Journal of Behavioral Medicine, 35(2), 179-189. doi: 10.1007/s10865-011-9338-4

Delahanty, L. M., Hayden, D., Meigs, J. B., Nathan, D. M., \& Williamson, D. A. (2002). Psychological and behavioral correlates of baseline BMI in the diabetes prevention program (DPP). Diabetes Care, 25(11), 1992-1998.

De Marco, M. \& Thorburn, S. (2009). The relationship between income and food insecurity among Oregon residents: does social support matter? Public Health Nutrition, 12(11), 2104-2112. doi:10.1017/S1368980009990243

Deshmuk-Taskar, P., Nicklas, T. A., Yang, S., \& Berenson, G. S. (2007). Does food group consumption vary by differences in socioeconomic, demographic, and lifestyle factors in young adults? The Bogalusa Heart Study. Journal of the American Dietetic Association, 107(2), 223-234. doi: 10.1016/j.jada.2006.11.004

Deshmukh-Taskar, P. R., O’Neil, C. E., Nicklas, T. A., Yang, S. J., Liu, Y., Gustat, J., \& Berenson, G. S. (2009). Dietary patterns associated with metabolic syndrome, sociodemographic and lifestyle factors in young adults: The Bogalusa Heart 
Study. Public Health Nutrition, 12(12), 2493-2503. doi:

$10.1017 /$ S1368980009991261

Detz, J. (2006). Your mouth craves fat. Joe Weider's Muscle \& Fitness, 67(3), 64.

Dilorio, C., Dudley, W. N., Soet, J., Watkins, J., \& Maibech, E. (2000). A social cognitive-based model for condom use among college students. Nursing Research, 49(4), 208-14.

Dingfelder, S. F. (2011). Reflecting on narcissism: Are young people more self-obsessed than ever before? American Psychological Association, 42(2), 64. Retrieved from http://www.apa.org/monitor/2011/02/narcissism.aspx

DiSantis, K. I., Grier, S. A., Odoms-Young, A., Baskin, M. L., Carter-Edwards, L., Young, D. R., Lassiter, V., \& Kumanyika, S. K. (2013). What "price" means when buying food: Insights from a multisite qualitative study with Black Americans. American Journal of Public Health, 103(3), 516-522. doi: 10.2105/AJPH.2012.301149. Retrieved from http://ajph.aphapublications.org/doi/full/10.2105/AJPH.2012.301149

Dodson, J. E. \& Gilkes, C. T. (1995). “There's nothing like church food”: Food and the U.S. Afro-Christian tradition: Re-membering community and feeding the embodied s/pirit(s). Journal of the American Academy of Religion, 63(3), 519538.

Drewnowski, A. \& Darmon, N. (2005). The economics of obesity: Dietary energy density and energy cost. The American Journal of Clinical Nutrition, 82(1), 265S-273S.

Drewnowski, A., Kurth, C., Holden-Wiltse, J., \& Saari, J. (1992). Food preferences in human obesity: Carbohydrates versus fats. Appetite, 18, 207-221.

Drewnowski, A. \& Specter, S. E. (2004). Poverty and obesity: The role of energy density and energy costs. The American Journal of Clinical Nutrition, 79(1), 6-16. 
Dudek, S. G. (2010). Nutrition essentials for nursing practice (6th ed.). Philadelphia: Wolters Kluwer Health/Lippincott Williams \& Wilkins.

Dudek, S. G. (2014). Nutrition essentials for nursing practice (7th ed.). Philadelphia: Wolters Kluwer Health/Lippincott Williams \& Wilkins.

Dumbrell, S. \& Mathai, D. (2008). Getting young men to eat more fruit and vegetables: A qualitative investigation. Health Promotion Journal of Australia, 19(3), 216-221.

Eckel, R. H. \& Krauss, R. M. (1998). American Heart Association call to action: Obesity as a major risk factor for coronary heart disease. Circulation, 97, 2099-2100. doi: 10.1161/01.CIR.97.21.2099

Edmonds, J. C. (2010). The relationship of stress to health-promoting behaviors of college-educated African American women. Bariatric Nursing and Surgical Patient Care, 5(4), 305-312. doi:10.1089/bar.2010.9992.

Edwards, P. J., Roberts, I., Clarke, M. J., DiGuiseppi, C., Wentz, R., Kwan, I., .., Pratap, S. (2009). Methods to increase response to postal and electronic questionnaires (Review). Cochrane Database of Systematic Reviews, Issue 3. doi:

10.1002/14651858.MR000008.pub4. Retrieved from http://onlinelibrary.wiley.com/doi/10.1002/14651858.MR000008.pub4/full

Ellison, C. G., Boardman, J. D., Williams, D. R., \& Jackson, J. S. (2001). Religious involvement, stress, and mental health: Findings from the 1995 Detroit Area Study. Social Forces, 80(1), 215-249. doi: 10.1353/sof.2001.0063

Ellison, C. G. \& Henderson, A. K. (2011). Religion and mental health: Through the lens of the stress process. In A. J. Blasi (Ed.), Toward a sociological theory of religion and health (pp. 11-44). Leiden: BRILL.

Ellison, C. G. \& Hummer, R. A. (Eds.). (2010). Religion, families, and health: Population-based research in the United States. New Brunswick, NJ: Rutgers University Press. 
Ellison, C. W. (1983). Spiritual well-being: Conceptualization and measurement. Journal of Psychology and Theology, 11(4), 330-340.

Ellison, C. W. \& Levin, J. S. (1998). The religion-health connection: Evidence, theory, and future directions. Health Education \& Behavior, 25(6), 700-720.

EMGO. (2012). Handling missing data. Retrieved from http://www.emgo.nl/kc/analysis/9\%20Handling\%20Missing\%20Data.html

Erdfelder, E., Faul, F., \& Buchner, A. (1996). GPOWER: A general power analysis program. Behavior Research Methods, Instruments, \& Computers, 28(1), 1-11.

Eyler, A. A., Haire-Joshu, D., Brownson, R. C., \& Nanney, M. S. (2004). Correlates of fat intake among urban, low income African Americans. American Journal of Health Behavior, 28(5), 410-417.

Facebook. (2013). Facebook. Retrieved from https://www.facebook.com

Fan, W. \& Han, Z. (2010). Factors affecting response rates of the web survey: A systematic review. Computer in Human Behavior, 26, 132-139.

Feman, S. P. C., Nguyen, L. T., Quilty, M. T., Kerr, C. E., Nam, B. H., Conboy, L. A., ... Davis, R. B. (2008). Effectiveness of recruitment in clinical trials: An analysis of methods used in a trial for irritable bowel syndrome patients. Contemporary Clinical Trials, 29(2), 241-251.

Ferguson, K. M. \& Xie, B. (2012). Adult support and substance use among homeless youths who attend high school. Child Youth Care Forum, 41, 427-445. doi: 10.1007/s10566-012-9175-9

Fernandes, J., Arts, J., Dimond, E., Hirshberg, S., \& Lofgren, I. E. (2013) Dietary factors are associated with coronary heart disease risk factors in college students. Nutrition Research, 33(8), 647-652. doi: 10.1016/j.nutres.2013.05.013 
Feunekes, G. I., de Graaf, C., Meyboom, S., \& van Staveren, W. A. (1998). Food choice and fat intake of adolescents and adults: Associations of intakes within social networks. Preventive Medicine, 27(5), 645-656.

Field, A. (2005). Discovering statistics using SPSS (2nd ed.). Thousand Oaks, CA: Sage Publications.

Field, A. (2013). Discovering statistics using SPSS (4th ed.). Thousand Oaks, CA: Sage Publications.

Fila, S. A. \& Smith, C. (2006). Applying the Theory of Planned Behavior to healthy eating behaviors in urban Native American youth. International Journal of Behavioral Nutrition and Physical Activity, 3(11), 10 pages. Online publication. doi:10.1186/1479-5868-3-11. Retrieved from http://www.ncbi.nlm.nih.gov/pmc/articles/PMC1501033/.

Finke, R. \& Stark, R. (1998). Religious choice and competition. American Sociological Review, 63(5), 761-766.

Fitzgibbon, M. L., Stolley, M. R., Ganschow, P., Schiffer, L., Wells, A., Simon, N., \& Dyer, A. (2005). Results of a faith-based weight loss intervention for Black women. Journal of the National Medical Association, 97(10), 1393-1402.

Fitzgibbon, M. L., Stolley, M., Schiffer, L., Sharp, L., Singh, V., Van Horn, L., \& Dyer, A. (2008). Obesity Reduction Black Intervention Trial (ORBIT): Design and baseline characteristics. Journal of Women's Health, 17(7), 1099-1110. doi: 10.1089/jwh.2007.0614

Fitzpatrick, J. J. (Ed.). (1998). Encyclopedia of nursing research. New York, NY: Springer Publishing Company, Inc.

Flegal, K. M., Carroll, M. D., Kit, B. K., \& Ogden, C. L. (2012). Prevalence of obesity and trends in the distribution of body mass index among US adults, 1999-2010. 
Journal of the American Medical Association (JAMA), 307(5), 491-497.

doi:10.1001/jama.2012.39

Flood, V. M., Webb, K., Lazarus, R., \& Pang, G. (2000). Use of self-report to monitor overweight and obesity in populations: some issues for consideration. Australian and New Zealand Journal of Public Health, 24(1), 96-99.

Fowles, E. R., Stang, J., Bryant, M., \& Kim, S. (2012). Stress, depression, social support, and eating habits reduce diet quality in the first trimester in low-income women: A pilot study. Journal of the Academy of Nutrition and Dietetics, 112(10), 16191625. doi: 10.1016/j.jand.2012.07.002

Frasca, T., Ventuneac, A., Balan, I., \& Carballo-Diéguez, A. (2012). Inner contradictions among men who bareback. Qualitative Health Research, 22(7), 946-956. doi:

$10.1177 / 1049732312443592$

Freedman, D. S. (2011). Obesity-United States, 1988-2008. Morbidity and Mortality Weekly Report (MMWR), 60(1), 73-77. Retrieved from http://www.cdc.gov/mmwr/preview/mmwrhtml/su6001a15.htm?s_cid=su6001a15 $-\mathrm{W}$

French, S. A., Story, M., Neumark-Sztainer, D., Fulkerson, J. A., \& Hannan, P. (2001). Fast food restaurant use among adolescents: Associations with nutrient intake, food choices and behavioral and psychosocial variables. International Journal of Obesity, 25(12), 1823-1833.

Frenn, M. \& Malin, S. (2003). Diet and exercise in low-income culturally diverse middle school students. Public Health Nursing, 20(5), 361-368. doi: 10.1046/j.15251446.2003.20504.x

Frenn, M., Malin, S., \& Bansal, N. K. (2003). Stage-based interventions for low-fat diet with middle school students. Journal of Pediatric Nursing, 18(1), 36-44. doi: 10.1053/ jpdn.2003.6 
Frenn, M., Malin, S., Villarruel, A. M., Slaikeu, K., McCarthy, S., Freeman, J., \& Nee, E. (2005). Determinants of physical activity and low-fat diet among low income African American and Hispanic middle school students. Public Health Nursing, 22(2), 89-97. doi: 10.1111/j.0737-1209.2005.220202.x

Fryar, C. D., Carroll, M. D., \& Ogden, C. L. (2012). NCHS Health E-Stat: Prevalence of obesity among children and adolescents: United States, trends 1963-1965 through 2009-2010. Retrieved from

http://www.cdc.gov/nchs/data/hestat/obesity_child_09_10/obesity_child_09_10.ht $\mathrm{m}$

Gans, K. M., Burkholder, G. J., Risica, P. M., \& Lasater, T. M. (2003). Baseline fatrelated dietary behaviors of White, Hispanic, and Black participants in a cholesterol screening and education project in New England. Journal of the American Dietetic Association, 103(6), 699-706. doi: 10.1053/jada.2003.50135

Gans, K. M, Risica, P. M., Kirtania, U., Jennings, A., Strolla, L. O., Steiner-Asiedu, M., Hardy, N., \& Lasater, T. M. (2009). Dietary behaviors and portion sizes of black women who enrolled in SisterTalk and variation by demographic characteristics. Journal of Nutrition Education and Behavior, 41(1), 32-40. doi: 10.1016/j.jneb.2008.05.013

Gary, T. L., Baptiste-Roberts, K.,Gregg, E. W., Williams, D. E., Beckles, G. L. A., Miller, E. J., III, \& Engelgau, M. M. (2004). Fruit, vegetable and fat intake in a population-based sample of African Americans. Journal of the National Medical Association, 96(12), 1599-1605.

Genia, V. (2001). Evaluation of the Spiritual Well-Being Scale in a sample of college students. The International Journal for the Psychology of Religion, 11(1), 25-33. doi: 10.1207/S15327582IJPR1101_03

George, J. B. (2011). Nursing theories: The base for professional nursing practice (6th ed.). Upper Saddle River, NJ: Pearson Education. 
Giesbrecht, N. (1999). Reducing risks associated with drinking among young adults:

Promoting knowledge-based perspectives and harm reduction strategies. Addiction, 94(3), 353-355. doi: 10.1046/j.1360-0443.1999.9433535

Gillum, R. F., King, D. E., Obisesan, T. O., \& Koenig, H. G. (2008). Frequency of attendance at religious services and mortality in a U.S. national cohort. Annals of Epidemiology, 18(2), 124-129.

Golay, A. \& Bobbioni, E. (1997). The role of dietary fat in obesity. International Journal of Obesity, 21(Suppl 3), S2-S11.

Goodman, L. A. (1961). Snowball sampling. The Annals of Mathematical Statistics, 32(1), 148-170.

Goodwin, L. D. \& Leech, N. L. (2006). Understanding correlation: Factors that affect the size of r. The Journal of Experimental Education, 74(3), 251-266.

Gorber, S. C., Tremblay, M., Moher, D., \& Gorber, B. (2007). A comparison of direct vs. self-report measures for assessing height, weight and body mass index: A systematic review. Obesity Reviews, 8(4), 307-326. doi: 10.1111/j.1467789X.2007.00347.X

Gravetter, F. J. \& Wallnau, L. B. (2004). Statistics for the behavioral sciences (6th ed.). Belmon, CA: Wadsworth/Thomson Learning.

Green, J. \& Thorogood, N. (2009). Qualitative methods of health research (2nd ed.). Los Angeles: Sage Publications, Inc.

Greenfield, E. A. \& Marks, N. F. (2009). Violence from parents in childhood and obesity in adulthood: Using food in response to stress as a mediator of risk. Social Science \& Medicine, 68, 791-798. dio: 10.1016/j.socscimed.2008.12.004

Grembowski, D., Patrick, D., Diehr, P., Durham, M., Beresford, S., Kay, E., \& Hecht, J. (1993). Self-efficacy and health behavior among older adults. Journal of Health and Social Behavior, 34(2), 89-104. 
Griotmedia, LLC. (2014). Soulciti.com. Retrieved from http://www.soulciti.com

Grizzle, R. W. (2009). Occupational stress, dietary self-efficacy, eating habits and body composition in police officers. (Doctoral dissertation). Available from ProQuest Dissertations and Theses database. (AAT 3356372). Retrieved from http://media.proquest.com/media/pq/classic/doc/1786561911/fmt/ai/rep/NPDF?_s =pDRiOpbt\%2FuHqPctOKBSaKrZLeVk\%3D

Grubbs, L. \& Carter, J. (2002). The relationship of perceived benefits and barriers to reported exercise behaviors in college undergraduates. Family \& Community Health, 25(2), 76-84.

Hampl, J. S. \& Betts, N. M. (1995). Comparisons of dietary intake and sources of fat in low- and high-fat diets of 18 to 24-year olds. Journal of the American Dietetic Association, 95(8), 893-897.

Harcrow, M. S. (2010). Relationships between religiosity, spirituality \& health behaviors among college students. (Doctoral dissertation). Available from ProQuest Dissertations and Theses database. (AAT 3439814). Retrieved from http://acumen.lib.ua.edu/content/u0015/0000001/0000475/u0015_0000001_0000 475.pdf

Hargreaves, M. K., Schlundt, D. G., \& Buchowski, M. S. (2002). Contextual factors influencing the eating behaviours of African American women: A focus group investigation. Ethnicity \& Health, 7(3), 133-147. dio:

$10.1080 / 1355785022000041980$

Harrell, S. P. (2000). A multidimensional conceptualization of racism-related stress: Implications for the well-being of people of color. American Journal of Orthopsychiatry, 70(1), 42-57. dio 10.1037/h0087722

Harring, H. A., Montgomery, K., \& Hardin, J. (2010). Perceptions of body weight, weight management strategies, and depressive symptoms among US college 
students. Journal of American College Health, 59(1), 43-50. doi:

http://dx.doi.org/10.1080/07448481.2010.483705

Hart, A. H. Jr., Bowen, D. J., Kuniyuki, A., Hannon, P., \& Campbell, M. K. (2007). The relationship between the social environment within religious organizations and intake of fat versus fruits and vegetables. Health Education \& Behavior, 34(3), 503-516. doi: 10.1177/1090198106295916

Hart, A. Jr., Tinker, L., Bowen, D., Longton, G., \& Beresford, S. A. A. (2006). Correlates of fat intake behaviors in participants in the Eating for a Healthy Life Study. Journal of the American Dietetic Association, 106(10), 1605-1613. doi: 10.1016/j.jada.2006.07.006

Heaney, C. A. \& Israel, B. A. (2008). Social networks and social support. In K. Glanz, B. K. Rimer, \& K. Viswanath (Eds.), Health behavior and health education: Theory, research, and practice (4th ed., pp. 189-210). San Francisco, CA: Jossey-Bass.

Helsen, M., Vollebergh, W., \& Meeus, W. (2000). Social support from parents and friends and emotional problems in adolescence. Journal of Youth and Adolescence, 29(3), 319-335.

Henry, Y. M. \& Eastell, R. (2000). Ethnic and gender differences in bone mineral density and bone turnover in young adults: Effect of bone size. Osteoporosis International, 11, 512-517.

Herman, C. P. \& Polivy. (2010). Sex and gender differences in eating behavior. In J. C. Chrisler \& D. R. McCreary (Eds.), Handbook of gender research in psychology (pp. 455-469). New York, NY: Springer Science+Business Media, LCC.

Hermstad, A. K, Swan, D. W., Kegler, M. C., Barnette, J. K., \& Glanz, K. (2010). Individual and environmental correlates of dietary fat intake in rural communities: A structural equation model analysis. Social Science \& Medicine, 71(1), 93-101. doi:10.1016/j.socscimed.2010.03.028 
Hernandez, B. C. (2011). The Religiosity and Spirituality Scale for youth: Development and initial validation. (Doctoral dissertation). Retrieved from http://etd.lsu.edu/docs/available/etd-10142011115001/unrestricted/hernandez_diss.pdf

Hill, T. D., Burdette, A. M., \& Idler, E. L. (2011). Religious involvement, health status, and mortality risk. In R. A. Settersten, Jr. \& J. L. Angel (Eds.), Handbook of sociology of aging (pp.533-546). New York: Springer.

Hill, T., Ellison, C., Burdette, A., \& Musick, M. (2006). Religious involvement and healthy lifestyles: Evidence from the survey of Texas residents. Annals of Behavioral Medicine, 34, 217-222.

Hiza, H. A. B., Casavale, K. O., Guenther, P. M., \& Davis, C. A. (2013). Diet quality of Americans differs by age, sex, race/ethnicity, income, and education level. Journal of the Academy of Nutrition and Dietetics, 113(2), 297-306. doi: 10.1016/j.jand.2012.08.011

Holt, M. K. \& Espelage, D. L. (2007). Perceived social support among bullies, victims, and bully-victims. Journal of Youth and Adolescence, 36, 984-994. doi: 10.1007/s10964-006-9153-3

Horton, S. E. B. (2013a, September 13). Religion and health-promoting behaviors among emerging adults. Journal of Religion and Health, 54(1), 20-34. doi: 10.1007/s10943-013-9773-3

Horton, S. E. B. (2013b, October 7). [Factors influencing dietary fat intake among Black young adults: Pilot study]. Unpublished raw data.

Horton, S. E. B., Alvear, E., \& Horton, D. L. (2014). Health ministry partnerships: Creating a habit for health. Journal of Christian Nursing, 31(1), 28-34. doi: 10.1097/CNJ.0000000000000030

Howarth, N. C., Huang, T. T.-K., Roberts, S. B., \& McCrory, M. A. (2005). Dietary fiber and fat are associated with excess weight in young and middle-aged US adults. 
Journal of the American Dietetic Association, 105(9), 1365-1372. doi:

10.1016/j.jada.2005.06.001

Hsiao, P. Y., Mitchell, D. C., Coffman, D. L., Allman, R. M., Locher, J. L., Sawyer, P., ...Hartman, T. J. (2013). Dietary patterns and diet quality among diverse older adults: The University of Alabama at Birmingham Study of Aging. The Journal of Nutrition, Health \& Aging, 17(1), 19-25. doi: 10.1007/s12603-012-0082-4

Hu, F. B., Manson, J. E., Stampfer, M. J., Colditz, G., Liu, S., Solomon, C. G., \& Willett, W. C. (2001). Diet, lifestyle, and the risk of type 2 diabetes mellitus in women. The New England Journal of Medicine, 345(11), 790-797. doi: 10.1056/NEJMOa010492

Hu, F. B., Manson, J. E., \& Willett, W. C. (2001). Types of dietary fat and risk of coronary heart disease: A critical review. Journal of the American College of Nutrition, 20(1), 5-19.

Huang, Y., Song, W.O., Schemmel, R. A., \& Hoerr, S. M. (1994). What do college students eat? Food selection and meal pattern. Nutrition Research, 14(8), 11431153.

Huber, L. R. (2007). Validity of self-reported height and weight in women of reproductive age. Maternal and Child Health Journal, 11(2), 137-144. doi: 10.1007/s10995-006-0157-0

Huck, S. W. (2008). Reading statistics and research (5th ed.). Boston: Pearson Education.

Huff, R. M. \& Kline, M. V. (1999). Promoting health in multicultural populations: A handbook for practitioners. Thousand Oaks, CA: Sage Publications, Inc.

Huffington Post. (2013). U.S. college students feel super special about themselves: American freshman survey. Retrieved from http://www.huffingtonpost.com/2013/01/07/us-college-studentsconfidence_n_2426858.html 
Hughes, J. W., Tomlinson, A., Blumenthal, J. A., Davidson, J. D., Sketch, M. H. Jr., \& Watkins, L. L. (2004). Social support and religiosity as coping strategies for anxiety in hospitalized cardiac patients. Annals of Behavioral Medicine, 28(3), $179-185$.

Huh, J., Sami, M., Abramova, Z. S., Spruijt-Metz, D., \& Pentz, M. A. (2013). Cigarettes, culture, and Korean American emerging adults: An exploratory qualitative study. Western Journal of Nursing Research, 35(9), 1205-1221. doi: $10.1177 / 0193945913490840$

Huntington, A., Gilmour, J., Schluter, P., Tuckett, A., Bogossian, F., \& Turner, C. (2009). The Internet as a research site: Establishment of a web-based longitudinal study of the nursing and midwifery workforce in three countries. Journal of Advanced Nursing, 65(6), 1309-1317. doi: 10.1111/j.1365-2648.2009.04995.x

Hurt, B. (Filmmaker). (2013). Soul food junkies [Film]. United States: Public Broadcasting Service (PBS). Retreived from http://www.pbs.org/independentlens/soul-food-junkies/

Hutchinson, K. C. (1995). Factors that predict health-promoting lifestyle behaviors among African-American university students. (Doctoral dissertation). Available from ProQuest Dissertations and Theses database. (AAT 9639920).

Hyman, C. \& Handal, P. J. (2006). Definition and evaluation of religion and spirituality items by religious professionals: A pilot study. Journal of Religion and Health, 45(2), 264-282. doi: 10.1007/s10943-006-9015-z

IBM Corporation. (2011). Statistical Package for the Social Science (SPSS), version 20.

IBM Corporation. (n. d.). Point biserial correlations in SPSS. Retrieved from http://www01.ibm.com/support/docview.wss?uid=swg21476004

Inglis, V., Ball, K., \& Crawford, D. (2009). Does modifying the household food budget predict changes in the healthfulness of purchasing choices among low- and highincome women? Appetite, 52(2), 273-279. doi: 10.1016/j.appet.2008.10.005 
Institute of Medicine of the National Academies. (2010). Bridging the evidence gap in obesity prevention: A framework to inform decision making. Washington D. C.: The National Academies Press.

Irazusta, A., Hoyos, I., Irazusta, J., Ruiz, F., Díaz, E., \& Gil, J. (2007). Increased cardiovascular risk associated with poor nutritional habits in first-year university students. Nutrition Research, 27(7), 387-394. doi: 10.1016/j.nutres.2007.05.007

Jackson, E. S., Tucker, C. M., \& Herman, K. C. (2007). Health value, perceived social support, and health self-efficacy as factors in a health-promoting lifestyle. Journal of American College Health, 56(1), 69-74. dio: 10.3200/JACH.56.1.69-74

Jackson, L. A., Ervin, K. S., Gardner, P. D., \& Schmitt, N. (2001). Gender and the Internet: Women Communicating and Men Searching. Sex Roles, 44(5), 363.

James, D. C. S. (2004). Factors influencing food choices, dietary intake, and nutritionrelated attitudes among African Americans: Application of a culturally sensitive model. Ethnicity \& Health, 9(4), 349-367. doi: 10.1080/1355785042000285375

James, D. C. S. (2009). Cluster analysis defines distinct dietary patterns for AfricanAmerican men and women. Journal of the American Dietetic Association, 109(2), 255-262. doi: 10.1016/j.jada.2008.10.052

Jebb, S. A. \& Prentice, A. M. (2001). Physiological regulation of macronutrient balance. In P. Björntorp (Ed), International textbook of obesity (pp. 125-135). Chichester, UK: John Wiley \& Sons, Ltd.

Jen, K. L. C., Brogan, K., Washington, O. G. M., Flack, J. M., \& Artinian, N. T. (2007). Poor nutrient intake and high obese rate in an urban African American population with hypertension. Journal of the American College of Nutrition, 26(1), 57-65.

Jenkins, S. K. (2007). Low income African American adolescent girl's eating choices. (Doctoral dissertation). Retrieved from the University of Texas Libraries Digital Repository, http://repositories.lib.utexas.edu/handle/2152/3259 
Jenkins, S. \& Horner, S. D. (2005). Barriers that influence eating behaviors in adolescents. Journal of Pediatric Nursing, 20(4), 258-267.

Jensen, L. A. (2011). Bridging cultural and developmental approaches to psychology. Oxford, New York: Oxford University Press.

Jeong, E. \& Kim, K. (2007). Influence of stress on snack consumption in middle school girls. Nutrition Research and Practice, 1(4), 349-355.

Jia, H. \& Lubetkin, E. I. (2010). Trends in quality-adjusted life-years lost contributed by smoking and obesity. American Journal of Preventive Medicine, 38(2), 138-144.

Johnson, J. E., Weinert, C., \& Richardson, J. K. (1998). Rural residents' use of cardiac rehabilitation programs. Public Health Nursing, 15(4), 288-296.

Johnson, R. L. (2005). Gender Differences in Health-Promoting Lifestyles of African Americans. Public Health Nursing, 22(2), 130-137. doi: 10.1111/j.07371209.2005.220206.x

Jones, M. Y. (2012). Health and religious commitment among college students: The effects of health behavior, mental health, and social support. (Doctoral dissertation). Retrieved from https://digarchive.library.vcu.edu/bitstream/handle/10156/4083/Jones_Monica_Ph D.pdf? sequence $=1$

Jones, S. \& Fox, S. (2009). Pew Internet \& American Life Project: Generations online in 2009. Retrieved from http://www.floridatechnet.org/Generations_Online_in_2009.pdf

Joshi, P., Hardy, E., \& Hawkins, S. (2009). The role of religiosity in the lives of the lowincome population: A comprehensive review of the evidence (RTI Project Number: 0211824.000). Retrieved from U.S. Department of Health \& Human Services Office of the Assistant Secretary for Planning and Evaluation, http://aspe.hhs.gov/hsp/09/religiosity/report.shtml 
Juonala, M., Magnussen, C. G., Berenson, G. S., Venn, A., Burns, T. L., Sabin, M. A., ...Raitakari, O. T. (2011). Childhood adiposity, adult adiposity, and cardiovascular risk factors. The New England Journal of Medicine, 365, 18761885. doi: 10.1056/NEJMoa1010112

Kandiah, J., Yake, M., Jones, J., \& Meyer, M. (2006). Stress influences appetite and comfort food preferences in college women. Nutrition Research, 26(3), 118-123. doi:10.1016/j.nutres.2005.11.010

Keenan, N. L. \& Shaw, K. M. (2011). Coronary heart disease and stroke deaths - United States, 2006. Morbidity and Mortality Weekly Report (MMWR), 60(1), 62-66. Retrieved from http://www.cdc.gov/mmwr/preview/mmwrhtml/su6001a13.htm?s_cid=su6001a13 _W

Keith, T. Z. (2006). Multiple regression and beyond. Boston, MA: Pearson Education.

Kelsey, K. S., Kirkley, B. G., De Vellis, R. F., Earp, J. A., Ammerman, A. S., Keyserling, T. C., ... Simpson Jr., R. J. (1996). Social support as a predictor of dietary change in a low-income population. Health Education Research, 11(3), 383-395. doi: 10.1093/her/11.3.383

Kennedy, E. T., Bowman, S. A., \& Powell, R. (1999). Dietary-fat intake in the US population. Journal of the American College of Nutrition, 18(3), 207-212.

Kiernan, M., Moore, S. D., Schoffman, D. E., Lee, K., King, A. C., Taylor, C. B., ... Perri, M. G. (2012). Social support for healthy behaviors: Scale psychometrics and prediction of weight loss among women in a behavioral program. Obesity, 20(4), 756-764. doi: 10.1038/oby.2011.293

Kilbourne, B., Cummings, S., \& Levine, R. S. (2011). Does religion protect against psychological distress among chronically ill and poor women? In A. Blasi (Ed.), Toward a psychological theory of religion and health (pp. 95-114). Leiden, The Netherlands: Koninklijke Brill NV. 
Killien, M. G., Habermann, B., \& Jarrett, M. (2001). Influence of employment characteristics on postpartum mothers' health. Women \& Health, 33(1-2), 63-81. doi: 10.1300/J013v33n01_05

Kim, H. (2013). Statistical notes for clinical researchers: Assessing normal distribution (2) using skewness and kurtosis. Restorative Dentistry \& Endodontics, 38(1): 5254. doi: 10.5395/rde.2013.38.1.52

Kim, K. H., McIntosh, W. A., Kubena, K. S., \& Sobal, J. (2008). Religion, social support, food-related social support, diet, nutrition, and anthropometrics in older adults. Ecology of Food and Nutrition, 47(3), 205-228. doi: $10.1080 / 03670240802035068$

Kim, K. H., \& Sobal, J. (2004). Religion, social support, fat intake and physical activity. Public Health Nutrition, 7(6), 773-781. doi: 10.1079/PHN2004601

Kirkpatrick, S. I., Dodd, K. W., Reedy, J., \& Krebs-Smith, S. M. (2012). Income and race/ethnicity are associated with adherence to food-based dietary guidance among US adults and children. Journal of the Academy of Nutrition and Dietetics, 112(5), 624-635.e6. doi: 10.1016/j.jand.2011.11.012.

Kittler, P. G. \& Sucher, K. P. (2008). Food and culture (5th ed.). Belmont, CA: Thomson Higher Education.

Klaassen, D. W., McDonald, M. J., \& James, S. (2006). Advance in the study of religious and spiritual coping. In P. T. Wong \& L. C. J. Vong (Eds.), Handbook of multicultural perspectives on stress and coping (pp. 105-132). New York, NY: Springer+Business Media, Inc.

Koenig, H. G. (2002). The connection between psychoneuroimmunology and religion. In H. Koenig \& H. Cohen (Eds.), The link between religion and health (pp. 11-30). Oxford, New York: Oxford University Press, Inc. 
Koenig, H. G. (2012). Religion, spirituality, and health: The research and clinical implications. ISRN Psychiatry, 2012, 33 pages, Article ID 278730. Online publication. doi: 10.5402/2012/278730

Koenig, H. G. \& Büssing, A. (2010). The Duke University Religion Index (DUREL): A five-item measure for use in epidemiological studies. Religions, 1, 78-78. dio: 10.3390/rel1010078. Retrieved from www.mdpi.com/journal/religions.

Koenig, H. G., McCullough, M.E., Larson, D.B. (Eds.). (2001). Handbook of religion and health. New York, NY: Oxford University Press.

Koikkalainen, M., Mykkãnen, H., Erkkila, A., Julkunen, J., Saarinen, T., Pyorala, K., Uusitupa, M., \& Lappalainen, R. (1999). Difficulties in changing the diet in relation to dietary fat intake among patients with coronary heart disease.

European Journal of Clinical Nutrition, 53(2), 120-125.

Krauss, R. M., Deckelbaum, R. J., Ernest, N., Fisher, E., Howard, B. V., Knopp, R. H., ... Weinberg, R. (1996). Dietary guidelines for healthy American adults: A statement for health professionals from the Nutrition Committee, American Heart Association. Circulation, 94, 1795-1800. doi: 10.1161/01.CIR.94.7.1795

Kremer, W. (2013, January 4). Does confidence really breed success? BCC News Magazine. Retrieved from http://www.bbc.com/news/magazine-20756247

Kronsberg, S. S., Obarzanek, E., Affenito, S. G., Crawford, P. B., Sabry, Z. I., Schmidt, M., ... Barton, B. A. (2003). Macronutrient intake of Black and White adolescent girls over 10 years: The NHLBI Growth and Health Study. Journal of the American Dietetic Association, 103(7), 852-860. doi: 10.1053/jada.2003.50169

Krul, A. J., Daanen, H. A. M., \& Choi, H. (2010). Self-reported and measured weight, height and body mass index (BMI) in Italy, the Netherlands and North America. European Journal of Public Health, 21(4), 414-419. doi: 10.1093/eurpub/ckp228

Kulkarni, K. (2004). Food, Culture, and Diabetes in the United States. Clinical Diabetes, 22(4), 190-192. doi: 10.2337/diaclin.22.4.190 
Kumanyika, S. K. (2007). Environmental influences on childhood obesity: Ethnic and cultural influences in context. Physiology \& Behavior, 94(1), 61-70. doi: 10.1016/j.physbeh.2007.11.019

Kumanyika, S. K., Morsink, C., \& Agurs, T. (1992). Models for dietary and weight change in African-American women: Identifying cultural components. Ethnicity \& Disease, 2(2), 166-75.

Kuzma, J. W. \& Bohnenblust, S. E. (2005). Basic statistics for the health sciences (5th ed.). New York, NY: McGraw Hill.

Lagou, V., Liu, G., Zhu, H., Stallmann-Jorgensen, I. S., Gutin, B., Dong, Y., \& Snieder, H. (2011). Lifestyle and socioeconomic-status modify the effects of ADRB2 and NOS3 on adiposity in European-American and African-American adolescents. Obesity, 19(3), 595-603. doi:10.1038/oby.2010.224

Landrine, H., Klonoff, E. A., Corral, I., Fernandez, S., \& Roesch, S. (2006). Conceptualizing and measuring ethic discrimination in health research. Journal of Behavioral Medicine, 29(1), 79-94. doi: 10.1007/s10865-005-9029-0

Laroche, H. H., Wallace, R. B., Snetselaar, L., Hillis, S. L., \& Steffen, L. M. (2012). Changes in diet behavior when adults become parents. Journal of the Academy of Nutrition and Dietetics, 112(6), 832-839. doi: 10.1016/j.jand.2012.02.024

Larson, D. B. \& Larson, S. S. (2003). Spirituality's potential relevance to physical and emotional health: A brief review of quantitative research. Journal of Psychology and Theology, 31(1), 37-51.

Larson, N., Neumark-Sztainer, D., Harwood, E. M., Eisenberg, M. E., Wall, M. M., \& Hannan, P. J. (2011). Do young adults participate in surveys that 'go green'? Response rates to a web and mailed survey of weight-related health behaviors. International Journal of Child Health and Human Development, 4(2), 225-231.

Lazarus, R. S. \& Folkman, S. (1984). Stress, appraisal, and coping. New York: Springer. 
Ledikwe, J. H., Smiciklas-Wright, H., Mitchell, D. C., Jensen, J. L., Friedmann, J. M., \& Still, C. D. (2003). Nutritional risk assessment and obesity in rural older adults: A sex difference. The American Journal of Clinical Nutrition, 77(3), 551-558.

Lehhart, A., Purcell, K., Smith, A., \& Zickuhr, K. (2010). Social media and young adults: Social media and mobile internet use among teens and young adults. Pew Internet \& American Life Project. Retrieved from http://www.pewinternet.org/Reports/2010/Social-Media-and-Young-Adults.aspx

Levi, J., Segal, L. M., St. Laurent, R., Rayburn, J. (2014). The state of obesity: Better policies for a healthier America 2014. Retrieved from http://www.rwjf.org/content/dam/farm/reports/reports/2014/rwjf414829

Levin, J., Chatters, L. M., \& Taylor, R. J. (2005). Religion, health and medicine in African Americans: Implications for physicians. Journal of the National Medical Association, 97(2), 237-249.

Lewis, I., Watson, B., \& White, K. M. (2009). Internet versus paper-and-pencil survey methods in psychological experiments: Equivalence testing of participant responses to health-related messages. Australian Journal of Psychology, 61(2), 107-116. doi: 10.1080/00049530802105865

Lewis, S. M., Mayhugh, M. A., Freni, S. C., Cardoso, S. S., Buffington, C., Jairaj, K., Turturro, A., \& Feuers, R. J. (2003). Assessment and significance of 24-h energy intake patterns among young and aged non-affluent southern US women. The Journal of Nutrition, Health \& Aging, 7(2), 78-83.

Liburd, C. (2003). Food, identity, and African-American women with type 2 diabetes: An anthropological perspective. Diabetes Spectrum, 16(3), 160-165. doi: 10.2337/diaspect.16.3.160

Lichtenstein, A. H. (1999). Dietary fat: A history. Nutrition Reviews, 57(1), 11-14.

Lichtenstein, A. H., Kennedy, E., Barrier, P., Danford, D., Ernst, N. D., Grundy, S. M., Leveille, G. A., Van Horn, L., Williams, C. L., \& Booth, S. L. (1998). Dietary fat 347 
consumption and health. Nutrition Reviews, 56(5), S3-19. doi: 10.1111/j.17534887.1998.tb01728.x

Lim, L. L., Seubsman, S., \& Sleigh, A. (2009). Validity of self-reported weight, height, and body mass index among university students in Thailand: Implications for population studies of obesity in developing countries. Population Health Metrics, 7(15), 8 pages. Online publication. doi:10.1186/1478-7954-7-15. Retrieved from http://www.pophealthmetrics.com/content/7/1/15

Lissner, L. \& Heitmann, B. L. (1995). Dietary fat and obesity: Evidence from epidemiology. European Journal of Clinical Nutrition, 49(2), 79-90.

Lissner, L., Levisky, D. A., Strupp, B. J., Kalfwarf, H. J., \& Roe, D. A. (1987). Dietary fat and the regulation of energy intake in human subjects. The American Journal of Clinical Nutrition, 46(6), 886-892.

Little, R. J. A. \& Rubin, D. B. (1987). Statistical analysis with missing data. New York: John Wiley \& Sons, Inc.

Liu, K., Daviglus, M. L., Loria, C. M., Colangelo, L. A., Spring, B., Moller, A. C., \& Lloyd-Jones, D. M. (2012). Healthy lifestyle through young adulthood and the presence of low cardiovascular disease risk profile in middle age: The Coronary Artery Risk Development in (Young) Adults (CARDIA) Study. Circulation, 125, 996-1004. doi: 10.1161/CIRCULATIONAHA.111.060681

Liu, Y., Gao, J. H., Liu, H. I., \& Fox, P. T. (2000). The temporal response of the brain after eating revealed by functional MRI. Nature, 405, 1058-1062. dio: $10.1038 / 35016590$

Lloyd, H. M., Paisley, C. M., \& Mela, D. J. (1995). Barriers to the adoption of reducedfat diets in a UK population. Journal of the American Dietetic Association, 95(3), 316-322. 
Lucan, S. C., Barag, F. K., Karasz, A., Palmer, C. S., \& Long, J. A. (2012). Perceived influences on diet among urban, low-income African Americans. American Journal of Health Behavior, 36(5), 700-710. doi: 10.5993/AJHB.36.5.12.

Luke, A., Cooper, R. S., Prewitt, T. E., Adeyemo, A. A., \& Forrester, T. E. (2001). Nutritional consequences of the African diaspora. Annual Review of Nutrition, 21, 47-71. doi: 10.1146/annurev.nutr.21.1.47

Lyon, B. L. (2000). Stress, coping, and health. In V. H. Rice (Ed.), Handbook of stress, coping, and health: Implications for nursing research, theory, and practice (pp. 3-26). Thousand Oaks, CA: Sage Publications.

MacCallum, R. C. Browne, M. W., \& Sugawara, H. M. (1996). Power analysis and determination of sample size for covariance structure modeling. Psychological Methods, 1(2), 130-149.

MacKinnon, D. P. \& Dwyer, J. H. (1993). Estimating mediated effects in prevention studies. Evaluation Review, 17(2), 144-158. doi: 10.1177/0193841X9301700202

Maskarinec, G., Novotny, R., \& Tasaki, K. (2000). Dietary patterns are associated with body mass index in multiethnic women. The Journal of Nutrition, 130(12), 30683072.

Martinelli, A. M. (1999). Testing a model of avoiding environmental tobacco smoke in young adults. Journal of Nursing Scholarship, 31(3), 237-242.

Mayo Clinic. (2013). Healthy diet: Do you follow dietary guidelines? Retrieved from http://www.mayoclinic.com/health/how-to-eat-healthy/MY02264

Mays, V. M., Cochran, S. D., \& Barnes, N. W. (2007). Race, race-based discrimination, and health outcomes among African Americans. Annual Review Of Psychology, 58, 201-225. doi: 10.1146/annurev.psych.57.102904.190212

Mazur, R. E., Marquis, G. S., \& Jensen, H. H. (2003). Diet and food insufficiency among Hispanic youths: Acculturation and socioeconomic factors in the third National 
Health and Nutrition Examination Survey. The American Journal of Clinical Nutrition, 78, 1120-1127.

McCann, B. S., Warnick, G. R., \& Knopp, R. H. (1990). Changes in plasma lipids and dietary intake accompanying shifts in perceived workload and stress. Psychosomatic Medicine, 52, 97-108.

McCoy, William (2013). The average BMI in the USA. Retrieved from http://www.livestrong.com/article/154837-the-average-bmi-in-the-usa/

McCoy, W. (2015). How effective is Craiglist? Houston Chronicle. Retrieved from http://smallbusiness.chron.com/effective-craigslist-26595.html

McDougle, L., Handy, F., Konrath, S., \& Walk, M. (2013). Health outcomes and volunteering: The moderating role of religiosity. Social Indicators Research. Online publication. doi: 10.1007/s11205-013-0336-5. Retrieved from http://works.bepress.com/lindseymcdougle/2/

McElligott, D., Siemers, S., Thomas, L., \& Kohn, N. (2009). Health promotion in nurses: Is there a healthy nurse in the house? Applied Nursing Research, 22, 211-215. doi: 10.1016/j.apnr.2007.07.005

McEwen, B. S. (1998). Protective and damaging effects of stress mediators. The New England Journal of Medicine, 338(3), 171-179.

McGee, B. B., Richardson, V., Johnson, G. S., \& Johnson, C. (2014). Qualitative study among African American parents to inform an intervention to promote adoption of the Dietary Guidelines for Americans Food and Physical activity recommendations. Food and Nutrition Sciences, 5(9), 835-849. dio: 10.4236/fns.2014.59093

McKinley, C. J. (2009). Investigating the influence of threat appraisals and social support on healthy eating behavior and drive for thinness. Health Communication, 24(8), 735-745. doi: 10.1080/10410230903264303 
McKnight, P. E., McKnight, K. M., Sidani, S., \& Figueredo, A. J. (2007). Missing data: A gentle introduction. New York, NY: The Guilford Press.

McNamara, P., Burns, J. P., Johnson, P., \& McCorkle, B. H. (2010). Personal religious practice, risky behavior, and implementation intentions among adolescents. Psychology of Religion and Spirituality, 2(1), 30-34.

Merrill, R. M., \& Thygerson, A. L. (2001). Religious preference, church activity, and physical exercise. Preventive Medicine, 33(1), 38-45.

Miles, J. \& Shevlin, M. (2001). Applying regression and correlation: A guide for students and researchers. London: SAGE Publications.

Millen, B. E., Quatromoni, P. A., Franz, M. M., Epstein, B. E., Cupples, L., \& Copenhafer, D. L. (1997). Population nutrient intake approaches dietary recommendations: 1991 to 1995 Framingham Nutrition Studies. Journal of the American Dietetic Association, 97(7), 742-749.

Miller, D. B. \& O’Callaghan, J. P. (2002). Neuroendocrine aspects of the response to stress. Metabolism, 51(6 Suppl 1), 5-10. doi:10.1053/meta.2002.33184

Miller, P. E., Morey, M. C., Hartman, T. J., Snyder, D. C., Sloane, R., Cohen, H. J., \& Demark-Wahnefried, W. (2012). Dietary patterns differ between urban and rural older, long-term survivors of breast, prostate, and colorectal cancer and are associated with body mass index. Journal of the Academy of Nutrition and Dietetics, 112(6), 824-831. doi: 10.1016/j.jand.2012.02.021

Mills, H., Reiss, N., \& Dombeck, M. (2008, June 30). Social impact of stress. MentalHelp.net. Retrieved from https://www.mentalhelp.net/articles/socialimpact-of-stress/

Minnesota State University. (2015). Emerging adulthood. Retrieved from http://www.mnsu.edu/counseling/emergingadulthood.html 
Mintz, S. W. \& Du Bois, C. M. (2002). The anthropology of food and eating. Annual Review of Anthropology, 31, 99-119.

Misra, R. (2007). Knowledge, attitudes, and label use among college students. Journal of the American Dietetic Association, 107(12), 2130-2134. doi:

doi:10.1016/j.jada.2007.09.001

Montmayeur, J. \& le Coutre, J. (Eds.). (2010). Fat detection: Taste, texture, and post ingestive effects. Boca Raton, FL: Taylor and Francis Group, LLC.

Morowatisharifabad, M. \& Shirazi, K. K. (2007). Determinants of oral health behaviors among preuniversity (12th-grade) students in Yazd (Iran): An application of the Health Promotion Model. Family \& Community Health, 30(4), 342-350.

Morrell, J. S., Lofgren, I. E., Burke, J. D., \& Reilly, R. A. (2012). Metabolic syndrome, obesity and related risk factors among college men and women. Journal of American College Health, 60(1), 82-89. doi: 10.1080/07448481.2011.582208

Morse, K. L. \& Driskell, J. A. (2009). Observed sex differences in fast-food consumption and nutrition self-assessments and beliefs of college students. Nutrition Research, 29(3), 173-179. doi:10.1016/j.nutres.2009.02.004

Mulder, B. C., de Bruin, M., Schreurs, H., van Ameijden, E. J. C., \& van Woerkum, C. M. J. (2011). Stressors and resources mediate the association of socioeconomic position with health behaviours. BMC Public Health, 11(798). Online publication. Retrieved from http://www.biomedcentral.com/1471-2458/11/798

Munro, B. H. (Ed.). (2005). Statistical methods for health care research (5th ed.). Philadelphia, PA: Lippincott Williams \& Wilkins.

Musick, M. A. (2004). Survey of Texas adults, 2004 variable index and codebook. Unpublished manuscript, Department of Sociology, The University of Texas at Austin, Austin, Texas. 
Musick, M. \& Worthen, M. G. F. (2010). Religion and physical health among U. S. adults. In C. G. Ellison \& R. A. Hummer (Eds.), Religion, families, and health: Population-based research in the United States (pp. 248-272). New Brunswick, NJ: Rutgers University Press.

Musick, M. A., Traphagan, J. W., Koenig, H. G., \& Larson, D. B. (2000). Spirituality in physical health and aging. Journal of Adult Development, 7(2), 73-86.

Muzoora, C., Martin, J. N., \& Weiser, S. D. (2012). Food insecurity, depression and the modifying role of social support among people living with HIV/AIDS in rural Uganda. Social Science \& Medicine, 74(12), 2012-2019. doi:10.1016/j.socscimed.2012.02.033

Nabi, R. L. \& Thomas, J. (2012). The effects of reality-based television programming on diet and exercise motivation and self-efficacy in young adults. Health Communication, 28(7), 699-708. doi: 10.1080/10410236.2012.711510. Retrieved from http://www.tandfonline.com/doi/full/10.1080/10410236.2012.711510

National Heart Foundation of Australia (2003). A review of the relationship between dietary fat and overweight/obesity. Retrieved from http://www.heartfoundation.org.au/SiteCollectionDocuments/Dietary-fat-ovobReview.pdf

National Restaurant Association. (2012). What's hot in 2013: Chef survey. Retrieved from http://www.google.com/url?sa=t\&rct=j\&q=\&esrc=s\&source=web\&cd=2\&ved=0 CDIQFjAB\&url=http\%3A\%2F\%2Fwww.restaurant.org\%2FDownloads\%2FPDF s\%2FNews-

Research\%2FWhatsHotFood2013.pdf\&ei=BMXIUfvoCuGXyAHzj4GgCw\&usg =AFQjCNHFLMk1je0ZIQAAvF3d77vYr8S7lw\&bvm=bv.49405654,d.aWc\&cad $=\mathrm{rja}$ 
Nayga, R. M. Jr. (2000). Nutrition knowledge, gender, and food label use. Journal of Consumer Affairs, 34(1), 97-112. doi: 10.1111/j.1745-6606.2000.tb00086.x

Nettleton, J. A., Polak, J. F., Tracy, R., Burke, G. L., \& Jacobs, D. R. Jr. (2009). Dietary patterns and incident cardiovascular disease in the Multi-Ethnic Study of Atherosclerosis. The American Journal of Clinical Nutrition, 90(3), 647-654. doi: 10.3945/ajen.2009.27597

Neumark-Sztainer, D., Story, M., Perry, C., \& Casey, M. A. (1999). Factors influencing food choices of adolescents: Findings from focus-group discussions with adolescents. Journal of the American Dietetic Association, 99(8), 929-937.

Newby, P. K., Muller, D., Hallfrisch, J., Qiao, N., Andres, R., \& Tucker, K. L. (2003). Dietary patterns and changes in body mass index and waist circumference in adults. The American Journal of Clinical Nutrition, 77(6), 1417-1425.

Newport, F. (2006). Religion and social trends: Religion most important to Blacks, women, and older Americans. Retrieved from http://www.gallup.com/poll/25585/religion-most-important-blacks-women-olderamericans.aspx

Ng, D. M. \& Jeffery, R. W. (2003). Relationships between perceived stress and health behaviors in a sample of working adults. Health Psychology, 22(6), 638-642. doi: 10.1037/0278-6133.22.6.638

Nicklas, T. A., Farris, R. P., Myers, L., \& Berenson, G. S. (1995). Impact of meat consumption on nutritional quality and cardiovascular risk factors in young adults: The Bogalusa Heart Study. Journal of the American Dietetic Association, 95(8), 887-892.

Nicklas, T. A., Jahns, L., Bogle, M. L., Chester, D. N., Giovanni, M., Klurfeld, D. M., ...Tucker, K. L. (2013). Barriers and facilitators for consumer adherence to the dietary guidelines for Americans: The HEALTH study. Journal of the Academy of Nutrition and Dietetics, 113(10), 1317-1331. 
Nicklas, T. A., Myers, L., Reger, C., Beech, B., \& Berenson, G. S. (1998). Impact of breakfast consumption on nutritional adequacy of the diets of young adults in Bogalusa, Louisiana: Ethnic and gender contrasts. Journal of the American Dietetic Association, 98(12), 1432-1438.

Nothwehr, F. (2004). Attitudes and behaviors related to weight control in two diverse populations. Preventive Medicine, 39, 674-680. doi:10.1016/j.ypmed.2004.02.034

Obisesan, T., Livingston, I., Trulear, H. D., \& Gillum, F. (2006). Frequency of attendance at religious services, cardiovascular disease, metabolic risk factors and dietary intake in Americans: an age-stratified exploratory analysis. International Journal of Psychiatry in Medicine, 36(4), 435-448.

Office of the Surgeon General. (2007). Testimony before the Subcommittee on Education Reform Committee on Education and Workforce United States House of Representatives: The obesity crisis in America. Retrieved from http://www.surgeongeneral.gov/news/testimony/obesity07162003.htm

Oliver, G., \& Wardle, J. (1999). Perceived effects of stress on food choice. Physiology \& Behavior, 66(3), 511-515.

Oliver, G., Wardle, J., \& Gibson, E. L. (2000). Stress and food choice: A laboratory study. Psychosomatic Medicine, 62, 853-865.

Omondi, D.O., Othuon, L.O.A., \& Mbagaya, G.M. (2011). Can physical activity and dietary fat intake influence body mass index in a cross-sectional correlational design? World Academy of Science, Engineering and Technology, 57,1039-1042.

Pargament, K. I. (1997). The psychology of religion and coping: Theory, research, practice. New York, NY: Guilford Press.

Park, C. L., Edmondson, D., Hale-Smith, A., \& Blank, T. O. (2009).

Religiousness/spirituality and health behaviors in younger adult cancer survivors: Does faith promote a healthier lifestyle? Journal of Behavioral Medicine, 32(6), 582-591. doi: 10.1007/s10865-009-9223-6 
Pattillo-McCoy, M. (1998). Church culture as a strategy of action in the Black community. American Sociological Review, 63(6), 767-784.

Patrician, P. A. (2002). Multiple imputation for missing data. Research in Nursing \& Health, 25, 76-84. doi: 10.1002/nur.10015

Patterson, B. H., Harlan, L. C., Block, G., \& Kahle, L. (1995). Food choices of Whites, Blacks, and Hispanics: Data from the 1987 National Health Interview Survey. Nutrition and Cancer, 23(2), 105-119. doi:10.1080/01635589509514367

Pawlak, R. \& Colby, S. (2009). Benefits, barriers, self-efficacy and knowledge regarding healthy foods; perception of African Americans living in eastern North Carolina. Nutrition Research and Practice, 3(1), 56-63. doi: 10.4162/nrp.2009.3.1.56

Pearson, R., Turner, L. W., Young, M. E., \& Knickerbocker, C. (2007). Correlates of healthy eating and physical activity among students in grades 6-12. American Joumal of Health Studies, 22(3), 170-178.

Pender, N. J. (1996). Health promotion in nursing practice (3rd ed.). Stanford, CT: Appleton \& Lange.

Pender, N. J., Murdaugh, C. L., Parsons, M. A. (2002). Health promotion in nursing practice (4th ed.). Upper Saddle River, NJ: Prentice Hall.

Pender, N.J., Murdaugh, C.L. \& Parsons, M.A. (2006). Health promotion in nursing practice (5th ed.). Upper Saddle River, NJ: Pearson Education.

Pender, N.J., Murdaugh, C.L. \& Parsons, M.A. (2011). Health promotion in nursing practice (6th ed.). Upper Saddle River, NJ: Pearson Education.

Peters, R. M., Aroian, K. J., \& Flack, J. M. (2006). African American culture and hypertension prevention. Western Journal of Nursing Research, 28(7), 831-863. doi:10.1177/0193945906289332

Pew Research Center. (2009). A religious portrait of African-Americans. Retrieved from http://www.pewforum.org/2009/01/30/a-religious-portrait-of-african-americans/ 
Pew Research Center. (2013a). A rising share of young adults live in their parents' home. Retrieved from http://www.pewsocialtrends.org/2013/08/01/a-rising-share-ofyoung-adults-live-in-their-parents-home/

Pew Research Center. (2013b). Teen and young adult Internet use. Retrieved from http://www.pewresearch.org/millennials/teen-internet-use-graphic/

Pew Research Center. (2014). Mobile technology fact sheet. Retrieved from http://www.pewinternet.org/fact-sheets/mobile-technology-fact-sheet/

Pew Research Center. (2015). Teen and young adult Internet use. Retrieved from http://www.pewresearch.org/millennials/teen-internet-use-graphic/

Philip, W., James, T., Nelson, M., Ralph, A., \& Leather, S. (1997). Socioeconomic determinants of health: The contribution of nutrition to inequalities in health. BMJ, 314, 1545-1549.

Piderman, K. M., Schneekloth, T. D., Pankratz, V. S., Maloney, S. D., \& Altchuler, S. I. (2007). Spirituality in alcoholics during treatment. The American Journal on Addictions, 16(3), 232-237. doi: 10.1080/10550490701375616

Polit, D. F. \& Beck, C. T. (2012). Nursing research: Generating and assessing evidence for nursing practice (9th ed.). Philadelphia, PA: Wilters Kluwer Health/Lippincott Williams \& Wilkins.

Polzer, R. L. \& Miles, M. S. (2007). Spirituality in African Americans with diabetes: Self-management through a relationship with God. Qualitative Health Research, 17(2), 176-188. doi: 10.1177/1049732306297750

Povey, R., Conner, M., Sparks, P., James, R., \& Shepherd, R. (2000). Application of the theory of planned behaviour to two dietary behaviours: Roles of perceived control and self-efficacy. British Journal of Health Psychology, 5(2), 121-139. doi: 10.1348/135910700168810 
Powell, L. H., Shahabi, L., \& Thoresen, C. E. (2003). Religion and spirituality: Linkages to physical health. American Psychologist, 58(1), 36-52. doi: 10.1037/0003066X.58.1.36

Preacher, K. J., \& Hayes, A. F. (2004). SPSS and SAS procedures for estimating indirect effects in simple mediation models. Behavior Research Methods and Instrumentation, 36(4), 717-731.

Purcell, K. (2011 November 2). Part III. Do people use the apps they download? Retrieved from http://www.pewinternet.org/2011/11/02/part-iii-do-people-usethe-apps-they-download/

Purcell, K., Entner, R., \& Henderson, N. (2010 September 14). The rise of apps culture. Retrieved from http://www.pewinternet.org/2010/09/14/the-rise-of-apps-culture/

Qualman, E. (2013). Socialnomics: How social media transforms the way we live and do business (2nd ed.). Hoboken, NJ: John Wiley \& Sons, Inc.

Qualtrics. (2014). Retrieved from http://www.qualtrics.com

Raffensperger, S., Kuczmarski, M. F., Hotchkiss, L., Cotugna, N., Evans, M. K., \& Zonderman, A. B. (2010). The effect of race and predictors of socioeconomic status on diet quality in the Healthy Aging in Neighborhoods of Diversity across the Life Span (HANDLS) study sample. Journal of the National Medical Association,102(10), 923-930.

Ramo, D. E. \& Prochaska, J. J. (2012). Broad reach and targeted recruitment using Facebook for an online survey of young adult substance use. Journal of Medical Internet Research, 14(1), e28. Retrieved from http://www.jmir.org/2012/1/e28/

Random.org. (2012). True random number generator. Retrieved from http://www.random.org 
Rees, C. A., Karter, A. J., \& Young, B. A. (2010). Race/ethnicity, social support, and associations with diabetes self-care and clinical outcomes in NHANES. The Diabetes Educator, 36(3), 435-444. doi: 10.1177/0145721710364419

Reeves, R. R., Adams, C. E., Dubbert, P. M., Hickson, D. A., \& Wyatt, S. B. (2012). Are religiosity and spirituality associated with obesity among African Americans in the southeastern United States (The Jackson Heart Study)? Journal of Religion and Health, 51(1), 32-48. doi: 10.1007/s10943-011-9552-y

Reuters. (2013). 77 percent of people use their computers while watching tv: Survey.

Retrieved from Huffington Post website: http://www.huffingtonpost.com/2013/04/09/tv-multitasking_n_3040012.html

Rew, L., Arheart, K. L., Thompson, S., \& Johnson, K. (2013). Predictors of adolescents' health-promoting behaviors guided by primary socialization theory. Journal for Specialists in Pediatric Nursing, 18(4), 277-288. doi: 10.1111/jspn.12036

Rew, L., \& Wong, Y. J. (2006). A systematic review of associations among religiosity/spirituality and adolescent health attitudes and behaviors. Journal of Adolescent Health, 38(4), 433-442. doi:10.1016/j.jadohealth.2005.02.004

Rew, L., Wong, Y. J., Torres, R., \& Howell, E. (2007). A linguistic investigation of mediators between religious commitment and health behaviors in older adolescents. Issues in Comprehensive Pediatric Nursing, 30(3), 71-86. doi: $10.1080 / 01460860701525147$

Rice, V. H. (Ed.). (2000). Handbook of stress, coping, and health:

Implications for nursing research, theory, and practice. Thousand Oaks, CA: Sage Publication, Inc.

Ritchie, L. D., Spector, P., Stevens, M. J., Schmidt, M. M., Schreiber, G. B., StriegelMoore, R. ,...\& Crawford, P. B. (2007). Dietary patterns in adolescence are related to adiposity in young adulthood in black and white females. The Journal of Nutrition, 137(2), 399-406. 
Roberts, K. A. \& Yamane, D. (2003). Religion in Sociological Perspective (4th ed.). Implications of one's definition of religion for conducting for research. Supplementary web materials for Chapter 1 . Retrieved from http://www.sagepub.com/rsp5e/study/resources/82986_01pe.pdf

Robinson, M. E. \& Hunter, P. H. (2001). Nutritional assessment of a predominately African-American inner-city clinic population. Wisconsin Medical Journal , 100(9), 32-38.

Roger, V. L., Go, A. S., Lloyd-Jones, D. M., Benjamin, E. J., Berry, J. D., Borden, W. B., ... Turner, M. B. (2011). Heart disease and stroke statistics--2012 update: A report from the American Heart Association. Circulation, 125, e2-e220. doi: 10.1161/CIR.0b013e31823ac046

Russinova, Z. \& Cash, D. (2007). Personal perspectives about the meaning of religion and spirituality among person with serious mental illnesses. Psychiatric Rehabilitation Journal, 30(4), 271-284. doi: 10.2975/30.4.2007.271.284

Ryan, T. P. (2013). Sample size determination and power. Hoboken, NJ: John Wiley \& Sons, Inc.

Sadler, G. R., Lee, H., Lim, R. S., \& Fullerton, J. (2010). Recruiting hard-to-reach United States population sub-groups via adaptations of snowball sampling strategy. Nursing \& Health Sciences, 12(3), 369-374. doi: 10.1111/j.14422018.2010.00541.x

Salganik, M. J. \& Heckathorn, D. D. (2004). Sampling and estimation in hidden populations using respondent-driven sampling. Sociological Methodology, 34(1), 193-239.

Sallis, J. F., Grossman, R. M., Pinski, R.B, Patterson, T. L., \& Nader, P. R. (1987). The development of scales to measure social support for diet and exercise behaviors. Preventive Medicine, 16, 825-836. 
Sallis, J. F., Pinski, R.B., Grossman, R. M., Patterson, T. L., \& Nader, P. R. (1988). The development of self-efficacy scales for health-related diet and exercise behaviors. Health Education Research, 3(3), 283-292. doi: 10.1093/her/3.3.283

Salmon, D. M. W. \& Flatt, J. P. (1985). Effect of dietary fat content on the incidence of obesity among ad libitum fed mice. International Journal of Obesity, 9, 443-449.

Sander, L. (2012). Colleges confront a gender gap in student engagement. Chronicle Of Higher Education, 59(10), B14-B17.

Satia, J. A., Galanko, J. A., \& Siega-Riz, A. M. (2004). Eating at fast-food restaurants is associated with dietary intake, demographic, psychosocial and behavioural factors among African Americans in North Carolina. Public Health Nutrition, 7(8), 10891096. doi: 10.1079/PHN2004662

Scheffer, J. (2002). Dealing with missing data. Research Letters in the Information and Mathematical Sciences, 3, 153-160. Retrieved from http://equinetrust.org.nz/massey/fms/Colleges/College\%20of\%20Sciences/IIMS/ RLIMS/Volume03/Dealing_with_Missing_Data.pdf

Schemmel, R., Mickelsen, O., \& Motawi, K. (1972). Conversion of dietary to body energy in rats as affected by strain, sex and ration. Journal of Nutrition, 102(9), 1187-1197.

Scholz, U., Ochsner, S., Hornung, R., \& Knoll, N. (2013). Does social support really help to eat a low-fat diet? Main effects and gender differences of received social support within the Health Action Process Approach. Applied Psychology: Health and Well-Being, 5(2), 270-290. doi: 10.1111/aphw.12010

Schlundt, D. G., Franklin, M. D., Patel, K., McClellan, L., Larson, C., Niebler, S., \& Hargreaves, M. (2008). Religious affiliation, health behaviors and outcomes: Nashville REACH 2010. American Journal of Health Behavior, 32(6), 714-724. doi: 10.5555/ajhb.2008.32.6.714 
Schwarzer, R. \& Renner, B. (2000). Social-cognitive predictors of health behavior: Action self-efficacy and coping self-efficacy. Health Psychology, 19(5), 487-495. doi: $10.1037 / 0278-6133.19 .5 .487$

Sellers, R. M., Caldwell, C. H., Schmeelk-Cone, K. H., \& Zimmerman, M. A. (2003). Racial identity, racial discrimination, perceived stress, and psychological distress among African American young adults. Journal of Health and Social Behavior, 44(3), 302-317.

Sellers, R. M. \& Shelton, J. N. (2003). The role of racial identity in perceived racial discrimination. Journal of Personality and Social Psychology, 84(5), 1079-1092. doi: 10.1037/0022-3514.84.5.1079

Selye, H. A. (1974). Stress without distress. Philadelphia: J. B. Lippincott Company.

Shankar, P., Dilworth, J. E. L., \& Cone, D. (2004). Dietary intake and health behavior among Black and White college females. Family and Consumer Sciences Research Journal, 33(2), 159-171. doi: 10.1177/1077727X04269613

Sharma, S., Cao, X., Arcan, C., Mattingly, M., Jennings, S., Song, H. J., \& Gittelsohn, J. (2009). Assessment of dietary intake in an inner-city African American population and development of a quantitative food frequency questionnaire to highlight foods and nutrients for a nutritional invention. International Journal of Food Sciences and Nutrition, 60(Suppl 5), 155-167. doi:10.1080/09637480902755061

Siewe, Y. J. (1999). Stages on limiting dietary-fat intake: Implications for African Americans. Journal of Black Studies, 29(5), 731-746.

Sigrist, L. D., Anderson, J. E., \& Auld, G. W. (2005). Senior military officers' educational concerns, motivators and barriers for healthful eating and regular exercise. Military Medicine, 170(10), 841-845

Sijtsma, F. P. C., Meyer, K. A., Steffen, L. M., Shikany, J. M., Van Horn, L., Harnack, L., Kromhout, D., \& Jacobs, D. R. Jr. (2012). Longitudinal trends in diet and 362 
effects of sex, race, and education on dietary quality score change: The Coronary Artery Risk Development in Young Adults Study. The American Journal of Clinical Nutrition, 95(3), 580-586. doi: 10.3945/ajcn.111.020719

Silverman, P., Hecht, L., \& McMillin, J.D. (2002). Social support and dietary change among older adults. Ageing and Society, 22, 29-59. doi:

$10.1017 / \mathrm{S} 0144686 \mathrm{X} 02008590$

Sims, R., Gordon, S., Garcia, W., Clark, E., Monye, D., Callender, C., \& Campbell, A. (2008). Perceived stress and eating behaviors in a community-based sample of African Americans. Eating Behaviors, 9(2), 137-142. doi:

10.1016/j.eatbeh.2007.06.006

Skitka, L. J. \& Sargis, E. G. (2006). The Internet as psychological laboratory. Annual Review of Psychology, 57, 529-555. doi: 10.1146/annurev.psych.57.102904.190048

Slining, M. M. \& Popkin, B. M. (2013). Trends in intakes and sources of solid fats and added sugars among U.S. children and adolescents: 1994-2010. Pediatric Obesity, 8(4), 307-324. doi: 10.1111/j.2047-6310.2013.00156.x

Smith, W.G. (June 2008). Does gender influence online survey participation?: A recordlinkage analysis of university faculty online survey response behavior. (ERIC Number: 501717) Retrieved from http://files.eric.ed.gov/fulltext/ED501717.pdf.

Smith, A. M. \& Owen, N. (1992). Associations of social status and health-related beliefs with dietary fat and fiber densities. Preventive Medicine, 21(6), 735-745.

Spencer, L. (2002). Results of a heart disease risk-factor screening among traditional college students. Journal of American College Health, 50(6), 291-296. doi: $10.1080 / 07448480209603447$

Sobel, M. E. (1982). Asymptotic confidence intervals for indirect effects in structural equation models. Sociological Methodology, 13, 290-312. 
Steptoe, A., Lipsey, Z., \& Wardle, J. (1998). Stress, hassles and variations in alcohol consumption, food choice and physical exercise: A diary study. British Journal of Health Psychology, 3(5), 1-63.

Stommel, M. \& Schoenborn, C. A. (2009). Accuracy and usefulness of BMI measures based on self-reported weight and height: Findings from the NHANES \& NHIS 2001-2006. BMC Public Health, 9(421). Online publication. doi: 10.1186/14712458-9-421. Retrieved from http://www.biomedcentral.com/1471-2458/9/421

Storch, E. A., Roberti, J. W., Heidgerken, A. D., Storch, J. B., Lewin, A. B., Killiany, E. M., ...Geffken, G. R. (2004a). The Duke Religion Index: A psychometric investigation. Pastoral Psychology, 53(2), 175-181.

Storch, E. A., Strawser, M. S., \& Storch, J. B. (2004b). Two-week test-retest reliability of the Duke Religion Index. Psychological Reports, 94, 993-994. doi: 10.2466/pr0.94.3.993-994

Strawbridge, W. J., Shema, S. J., Cohen, R. D., \& Kaplan, G. A. (2001). Religious attendance increases survival by improving and maintaining good health behaviors, mental health, and social relationships. Annals of Behavior Medicine, 23(1), 68-74.

Strogatz, D.S., Croft, J. B., James, S. A., Keenan, N. L., Browning, S. R., Garrett, J. M., $\&$ Curtis, A. B. (1997). Social support, stress, and blood pressure in Black adults. Epidemiology, 8(5), 482-487.

Stuifbergen, A. K. (1999). Barriers and health behaviors of rural and urban persons with MS. American Journal of Health Behaviors, 23(6), 415-425.

Stunkard, A. J. \& Albaum, J. M. (1981). The accuracy of self-reported weights. The American Journal of Clinical Nutrition, 34(81), 1593-1599.

Subar, A. F., Ziegler, R. G., Patterson, B. H., Ursin, G., \& Graubard, B. (1984). US dietary patterns associated with fat intake: The 1987 National Health Interview Survey. American Journal of Public Health, 84(3), 359-366. 
Subrahmanyam, K., Reich, S. M., Waechter, N., \& Espinoza, G. (2008). Online and offline social networks: Use of social networking sites by emerging adults. Journal of Applied Developmental Psychology, 29(6), 420-433.

Sue, V. M. \& Ritter, L. A. (2012). Conducting online surveys (2nd ed.). Thousand Oaks, CA: Sage Publications, Inc.

Tabachnick, B.G., \& Fidell, L.S. (2013). Using multivariate statistics (6th ed.). Boston, MA: Pearson.

Takamizawa, E. (1999). Religious commitment theory: A model for Japanese Christians. Torch Trinity Journal, 164-176. Retrieved from http://www.ttgst.ac.kr/upload/ttgst_resources13/20123-128.pdf

Tan, M., Chan, C. K. Y., \& Reidpath, D. D. (2013). Religiosity, spirituality and the intake of fruit, vegetable and fat: A systematic review. Evidence-Based Complementary and Alternative Medicine, 18 pages, Article ID 146214. Online publication. Retrieved from http://www.hindawi.com/journals/ecam/aip/146214/

Taylor, R. J. \& Chatters, L. M. (2010). Importance of religion and spirituality in the lives of African American, Caribbean Blacks, and Non-Hispanic Whites. The Journal of Negro Education, 79(3), 280-294.

Taylor, A. W., Dal Grande, E., Gill, T. K., Chittleborough, C. R., Wilson, D. H., Adams, R. J., ...Ruffin, R. E. (2006). How valid are self-reported height and weight? A comparison between CATI self-report and clinic measurements using a large cohort study. Australian and New Zealand Journal of Public Health, 30(3), 238246. doi: 10.1111/j.1467-842X.2006.tb00864.x

Taylor, H. A. Jr., Coady, S. A., Levy, D., Walker, E. R., Vasan, R. S., Liu, J., ...Fox, C. (2010).Relationships of BMI to cardiovascular risk factors differ by ethnicity. Obesity, 18(8), 1638-1645. doi:10.1038/oby.2009.407 
Taylor, S. E. \& Holden, K. B. (2009). The health status of Black women. In R. L. Braithwaite, S. E. Taylor, \& H. M. Treadwell (Eds.), Health issues in the Black community (3rd ed., pp. 55-71). San Francisco, CA: Jossey-Bass.

Tomiyama, A. J., Puterman, E., Epel, E. S., Rehkopf, D. H., \& Laraia, B. A. (2013). Chronic psychological stress and racial disparities in body mass index change between Black and White girls aged 10-19. Annals of Behavioral Medicine, 45(1), 3-12. doi: 10.1007/s12160-012-9398-x

Temple, N. J., Wilson, T., \& Jacobs, D. R. Jr. (Eds.). (2012). Nutritional health: Strategies for disease prevention (3rd ed.). New York: Springer Science+Business Media, LLC.

The Henry J. Kaiser Family Foundation. (2013). Summary of the Affordable Care Act. Retrieved from http://kff.org/health-reform/fact-sheet/summary-of-the-affordablecare-act/

Thompson, F.E., Mistune, D., Subaru, A.F., McNeel, T., Berrigan, D., \& Kipnis, V. (2005). Dietary estimates in the National Health Interview Survey, 2000: Methodology, results, and interpretation. Journal of the American Dietetic Association, 105(3), 352-363. doi: 10.1016/j.jada.2004.12.032

Thrasher, J. F., Campbell, M. K., \& Oates, V. (2004). Behavior-specific social support for healthy behaviors among African American church members: Applying Optimal Matching Theory. Health Education \& Behavior, 31, 193-205. doi: 10.1177/1090198103259184

Timmerman, G. M. (2007). Addressing barriers to health promotion in underserved women. Family \& Community Health, 30(1), S34-S42.

Timmerman, G. M. \& Brown, A. (2012). The effect of a mindful restaurant eating intervention on weight management in women. Journal of Nutrition and Education Behavior, 44(1), 22-28. doi: 10.1016/j.jneb.2011.03.143 
Toobert, D. J., Strycker, L. A., Hampson, S. E., Westling, E., Christiansen, S. M., Hurley, T. G., \& Hébert, J. R. (2011). Computerized portion-size estimation compared to multiple 24-hour dietary recalls for measurement of fat, fruit, and vegetable intake in overweight adults. Journal of the American Dietetic Association, 111, 15781583. doi: 10.1016/j.jada.2011.07.004

Torres, S. J. \& Nowson, C. A. (2007). Relationship between stress, eating behavior, and obesity. Nutrition, 23, 887-894. doi:10.1016/j.nut.2007.08.008

Traffic Pillar. (2008). Craigslist classifieds. Retrieved from http://trafficpillar.com/craigslist.php

Tremblay, A., Lavallee, N., Aaemeras, N., Allard, L., Despres, J., \& Bouchard, C. (1991). Nutritional determinants of the increase in energy intake associated with a high-fat diet. The American Journal of Clinical Nutrition, 53(5), 1134-1137.

Trust for America's Health. (2015). F as in fat: How obesity threatens America's future 2013. Retrieved from http://healthyamericans.org/report/108/

Tucker, C. M., Rice, K. G., Hou, W., Kaye, L. B., Nolan, S. E. M., Grandoit, D. J., ... Desmond, F. F. (2011). Development of the Motivators of and Barriers to HealthSmart Behaviors Inventory. Psychological Assessment, 23(2), 487-503. doi: $10.1037 / \mathrm{a} 0022299$

Tucker, S. J., Weymiller, A. J., Cutshall, S. M., Rhudy, L. M., \& Lohse, C. M. (2012). Stress ratings and health promotion practices among RNs: A case for action. The Journal of Nursing Administration, 42(5), 282-292. doi:

10.1097/NNA.0b013e318253585f

Turrell, G. (1997). Determinants of gender differences in dietary behavior. Nutrition Research, 17(7), 1105-1120.

U.S. Department of Agriculture. (2010). Report of the Dietary Guidelines Advisory Committee on the dietary guidelines for Americans, 2010. Retrieved from http://www.cnpp.usda.gov/dgas2010-dgacreport.htm 
U.S. Department of Health and Human Services. (2013a, August 28). Healthy People 2020: Heart disease and stroke. Retrieved from http://healthypeople.gov/2020/topicsobjectives2020/objectiveslist.aspx?topicId=2 1

U.S. Department of Health and Human Services. (2013b, November 13). Healthy People 2020: Nutrition and weight status. Retrieved from http://healthypeople.gov/2020/topicsobjectives2020/overview.aspx?topicid=29

U.S. Department of Health and Human Services. (2014, November 5). The Affordable Care Act and young adults. Retrieved from http://www.hhs.gov/healthcare/facts/factsheets/2014/11/affordable-care-actyoung-adults.html

U. S. House of Representatives Select Committee on Hunger. (1993). Urban grocery gap : Hearing before the Select Committee on Hunger, House of Representatives, One Hundred Second Congress, second session, hearing held in Washington, DC, September 30, 1992. Washington: Government Printing Office.

U.S. Congress Joint Economic Committee. (2010). Understanding the economy:

Unemployment among young workers. Retrieved from http://www.jec.senate.gov/public/?a=Files.Serve\&File_id=adaef80b-d1f3-479c97e7-727f4c0d9ce6

University of Texas at Austin. (2014a). Events calendar. Retrieved from http://calendar.utexas.edu

University of Texas at Austin. (2014b). Qualtrics survey tool. Retrieved from http://www.utexas.edu/its/survey

UyBico, S. J., Pavel, S., \& Gross, C. P. (2007). Recruiting vulnerable populations into research: A systematic review of recruitment interventions. Society of General Internal Medicine, 22, 852-863. doi: 10.1007/s11606-007-0126-3. 
Vachon, M., Fillion, L., \& Achille, M. (2009). A conceptual analysis of spirituality at the end of life. Journal of Palliative Medicine, 12(1), 53-59. doi:

10.1089/jpm.2008.0189

Vadiveloo, M., Scott, M., Quatromoni, P., Jacques, P., \& Parekh, N. (2014). Trends in dietary fat and high-fat food intakes from 1991 to 2008 in the Framingham Heart Study participants. British Journal of Nutrition, 111(4), 724-734. doi: $10.1017 / \mathrm{S} 0007114513002924$

Vernarelli, J. A., Mitchell, D. C., Hartman, T. J. \& Rolls, B. J. (2011). Dietary energy density is associated with body weight status and vegetable intake in U.S. children. The Journal of Nutrition, 141(12), 2204-2210. doi: 10.3945/ jn.111.146092

Vriendt, T. D., Huybrechts, I., Ottevaere, C., Van Trimpont, I., \& De Henauw, S. (2009). Validity of self-reported weight and height of adolescents, its impact on classification into BMI-categories and the association with weighing Behaviour. International Journal of Environmental Research and Public Health, 6, 26962711. doi: 10.3390/ijerph6102696

von Deneen, K. M. \& Liu, Y. (2011). Obesity as an addiction: Why do the obese eat more? Maturitas, 68, 342-345. dio: 10.1016/j.maturitas.2011.01.018

Waelde, L. C., Pennington, D., Mahan, C., Mahan, R., Kabour, M., \& Marquett, R. (2010). Psychometric properties of the Race-Related Events Scale. Psychological Trauma: Theory, Research, Practice, and Policy, 2(1), 4-11. doi: $10.1037 / \mathrm{a} 0019018$

Walcott-McQuigg, J. A. (1995). The relationship between stress and weight-control behavior in African-American women. Journal of the National Medical Association, 87(6), 427-432.

Waldron-Perrine, B., Rapport, L. J., Hanks, R. A., Lumley, M., Meachen, S. J., \& Hubbarth, P. (2011). Religion and spirituality in rehabilitation outcomes among 
individuals with traumatic brain injury. Rehabilitation Psychology, 56(2), 107116. doi: $10.1037 / \mathrm{a} 0023552$

Wallace, S. A., McLellan-Lemal, E., Harris, M. J., Townsend, T. G., \& Miller, K. S. (2011). Why take an hiv test? Concerns, benefits, and strategies to promote HIV testing among low-income heterosexual African American young adults. Health Education \& Behavior, 38(5), 462-470. doi: 10.1177/1090198110382501

Wang, Y. \& Beydoun, M. A. (2007). The obesity epidemic in the United States-gender, age, socioeconomic, racial/ethnic, geographic characteristics: A systematic review and meta-regression analysis. Epidemiologic Reviews, 29, 6-28. dio: 10.1093/epirev/mxm007

Wansink, B. (2006). Mindless eating: Why we eat more than we think. New York, NY: Bantam Dell.

Wansink, B. \& Sobal, J. (2007). Mindless eating: The 200 daily food decisions we overlook. Environment and Behavior, 39(1), 106-123. doi: $10.1177 / 0013916506295573$

Wardle, J., Steptoe, A., Oliver, G., \& Lipsey, Z. (2000). Stress, dietary restraint and food intake. Journal of Psychosomatic Research, 48, 195-202.

Warick, Z. S. \& Weingarten, H. P. (1995). Determinants of high-fat diet hyperphagia: Experimental dissection of orosensory and postingestive effects. The American Journal of Physiology, 269(1), R30-37.

Warner, R. (2013). Applied statistics: From bivariate to multivariate techniques (2nd ed.). Thousand Oaks, CA: Sage.

Watson, N. J. \& Nesti, M. (2005). The role of spirituality in sport psychology consulting: An analysis and integrative review of literature. Journal of Applied Sport Psychology, 17(3), 228-239. doi: 10.1080/10413200591010102 
Watters, J. L. \& Satia, J. A. (2009). Psychosocial correlates of dietary fat intake in African-American adults: A cross-sectional study. Nutrition Journal, 8(15). doi:10.1186/1475-2891-8-15

Weight-control Information Network. (2014). Overweight and obesity statistics. Retrieved from http://win.niddk.nih.gov/statistics/

Whitt-Glover, M. C., Hogan, P. E., Lang, W., \& Heil, D. P. (2008). Pilot study of a faithbased physical activity program among sedentary Blacks. Preventing Chronic Disease, 5(2), A51.

Wickrama, K. A., Ralston, P. A., O'Neal, C. W., Ilich, J. Z., Harris, C. M., Coccia, C., ...Lemacks, J. (2012). Life dissatisfaction and eating behaviors among older African Americans: The protective role of social support. Journal of Nutrition Health and Aging, 16(9), 749-53. doi: 10.1007/s12603-012-0404-6.

Wilks, S. E. (2008). Resilience amid academic stress: The moderating impact of social support among social work students. Advances in Social Work, 9(2), 106-125.

Wilson, D. K. \& Ampey-Thornhill, G. (2001). The role of gender and family support on dietary compliance in an African American Adolescent hypertension prevention study. Annals of Behavioral Medicine, 23(1), 59-67.

Wilson, T. A., Adolph, A. L., \& Butte, N. F. (2009). Nutrient adequacy and diet quality in non-overweight and overweight Hispanic children of low socioeconomic status: The Viva la Familia Study. Journal of the American Dietetic Association, 109(6), 1012-1021. doi: 10.1016/j.jada.2009.03.007

Wilson, M. (2005). Health-promoting behaviors of sheltered homeless women. Family \& Community Health, 28(1), 51-63.

Winkleby, M. A., Robinson, T. N., Sundquist, J., \& Kraemer, H. C. (1999). Ethnic variation in cardiovascular disease risk factors among children and young adults: Findings from the Third National Health and Nutrition Examination Survey, 
1988-1994. Journal of American Medical Association, 281(11), 1006-1013. doi:10.1001/jama.281.11.1006

Wright, J. D. \& Wang, C. (2010). Trends in intake of energy and macronutrients in adults from 1999-2000 through 2007-2008 (NCHS Data Brief No. 49). Retrieved from http://www.cdc.gov/nchs/data/databriefs/db49.htm

Vedhara, K. \& Irwin, M. (Eds.). (2005). Human psychoneuroimmunology. Oxford, United Kingdom: Oxford University Press.

Welsh, E. M., Jeffery, R. W., Levy, R. L., Langer, S. L., Flood, A. P., Jaeb, M. A., \& Laqua, P. S. (2012). Measuring perceived barriers to healthful eating in obese, treatment-seeking adults. Journal of Nutrition Education and Behavior, 44(6), 507-512.

Yancey, A. K., Ortega, A. N., \& Kumanyika, S. K. (2006). Effective recruitment and retention of minority research participants. Annual Review of Public Health, 27,128. doi: 10.1146/annurev.publhealth.27.021405.102113

Zamora, D., Gordon-Larsen, P., Jacobs, D. R. Jr., \& Popkin, B. M. (2010). Diet quality and weight gain among Black and White young adults: The Coronary Artery Risk Development in Young Adults (CARDIA) Study (1985-2005). The American Journal of Clinical Nutrition, 92(4), 784-793. doi: 10.3945/ajcn.2010.29161

ZipSurvey.com. (2009). Features. Retrieved from http://www.zipsurvey.com/Default.aspx

Zickuhr, K. (2011). Pew Research Center's Internet and American Life Project: Generations and their gadgets. Retrieved from http://www.pewinternet.org/files/oldmedia/Files/Reports/2011/PIP_Generations_and_Gadgets.pdf 


\section{Vita}

Shalonda Estelle-Brazzell Horton was born in Weatherford, Texas. Coming from humble beginnings, her parents instilled in her a solid work ethic and to treasure education, which pushed her to graduate with honors from Weatherford High School in 1991. After high school, she entered Texas Christian University, Fort Worth, Texas in the fall of 1991. Blessed by an Army ROTC Scholarship in 1992, she was able to successfully complete her Bachelor of Science in Nursing from Texas Christian University in June 1995. She took her oath of office and served her country as an active duty nurse in the United States Army Nurse Corps for over 4 years; and then she transferred to the United States Army Reserves for another 12 years of service. She is currently a Major in the Individual Ready Reserve (IRR).

Her nearly 20 years of diverse nursing experience, both military and civilian, include the following areas: public health, oncology, medical/surgical, inpatient psychology, outpatient surgery, and post anesthesia care unit. After encountering many patients, with preventable health conditions, she decided to shift her interests towards health promotion, prevention, and research. This newfound passion motivated her to pursue both a MSN (public health nursing) and PhD in nursing at the University of Texas

at Austin. Her concern for the health of younger generations inspired her to focus her research interest in factors that influence the health, specifically dietary behaviors, among emerging adults.

Address: shorton@mail.nur.utexas.edu 
This manuscript was typed by the author. 